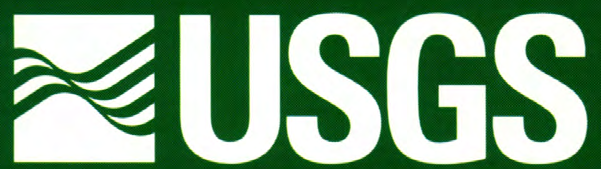

science for a changing world

Prepared in cooperation with the

UPPER BIG BLUE NATURAL RESOURCES DISTRICT

\title{
Hydrogeology and Subsurface Nitrate in the Upper Big Blue Natural Resources District, Central Nebraska, July 1995 through September 1997
}

U.S. GEOLOGICAL SURVEY

Water-Resources Investigations Report 98-4207

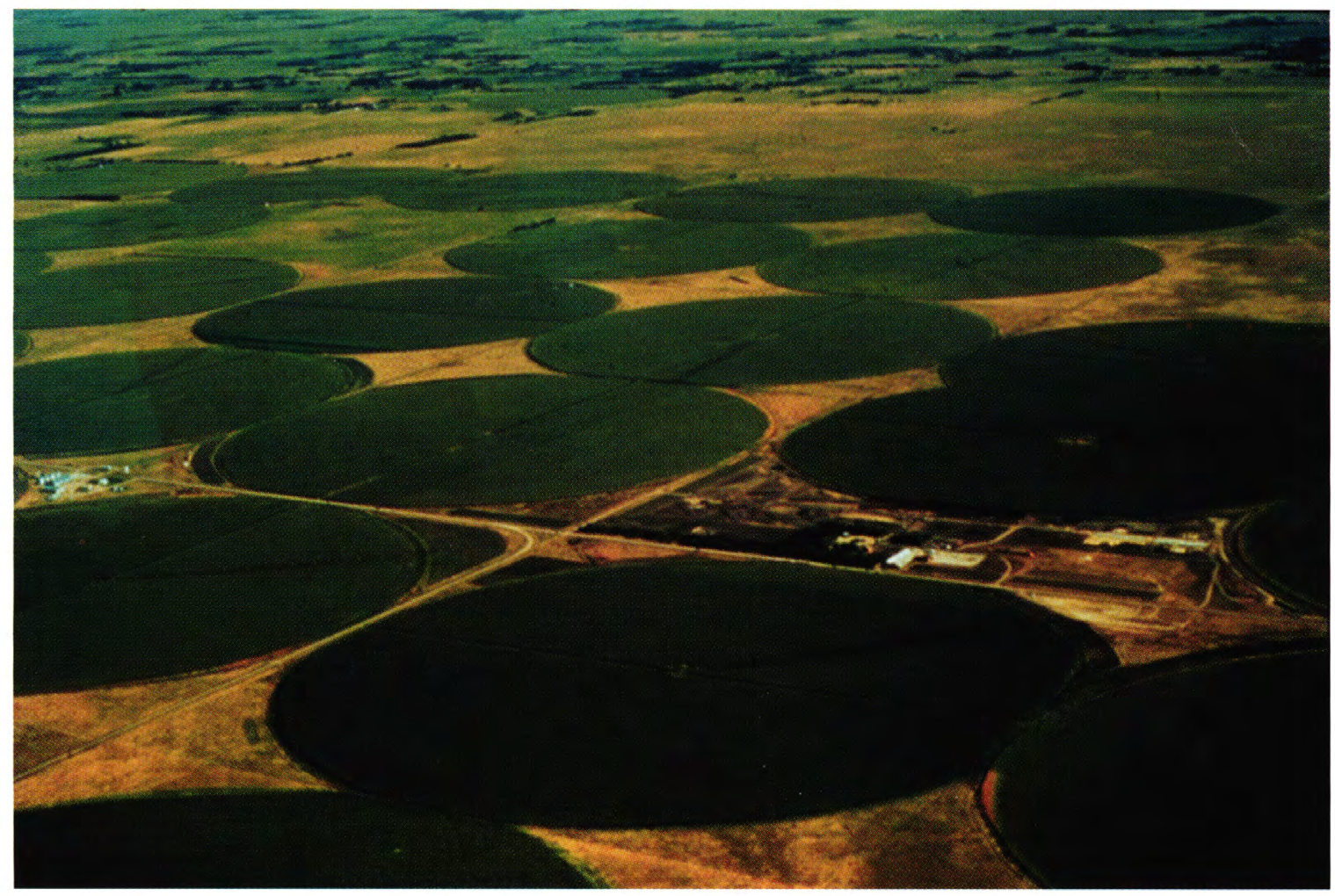

U.S. Department of the Interior

U.S. Geological Survey 
U.S. Department of the Interior

U.S. Geological Survey

Hydrogeology and Subsurface Nitrate in the Upper Big Blue Natural Resources District, Central Nebraska, July 1995 through September 1997

By I.M. Verstraeten, V.L. McGuire, and K.L. Heckman

Water-Resources Investigations Report 98-4207

Prepared in cooperation with the

Upper Big Blue Natural Resources District 


\section{U.S. DEPARTMENT OF THE INTERIOR}

Bruce Babbitt, Secretary

U.S. GEOLOGICAL SURVEY

Charles G. Groat, Director

Any use of trade, product, or firm names in this publication is for descriptive purposes only and does not imply endorsement by the U.S. Government.

Lincoln, Nebraska: 1998

For additional information write to:

District Chief

U.S. Geological Survey, WRD

406 Federal Building

100 Centennial Mall North

Lincoln, NE 68506

Copies of this report can be purchased from:

U.S. Geological Survey

Branch of Information Services

Box 25286

Denver, CO 80225-0286 


\section{CONTENTS}

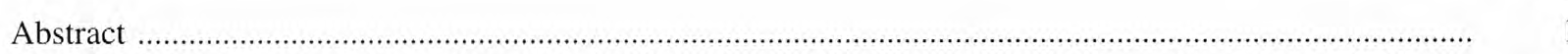

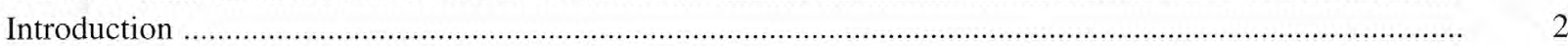

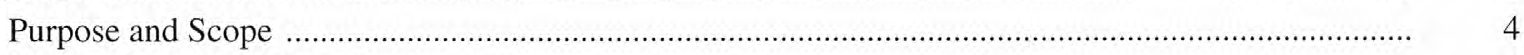

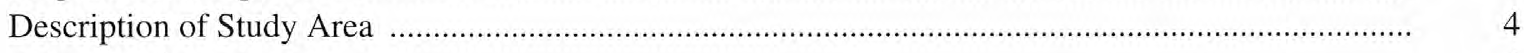

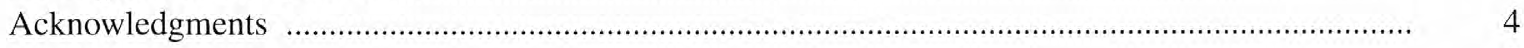

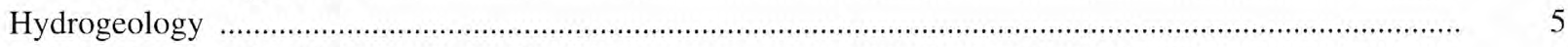

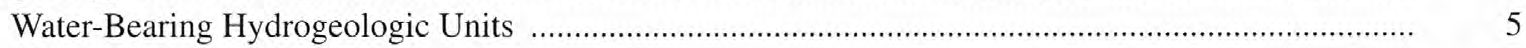

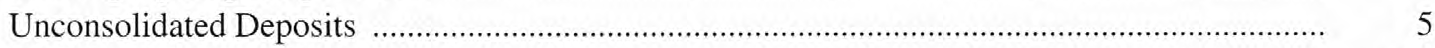

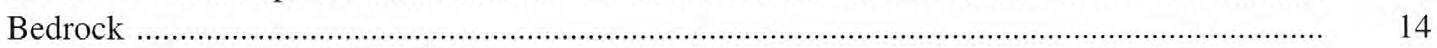

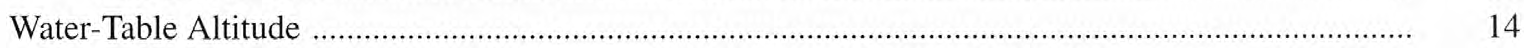

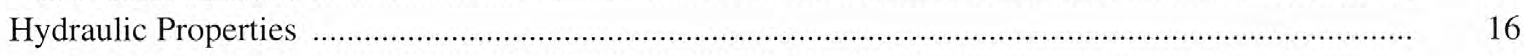

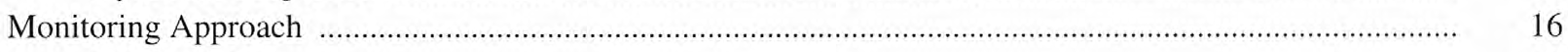

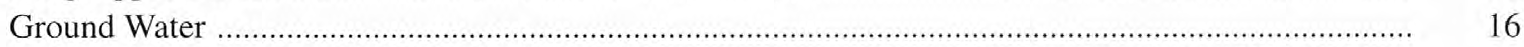

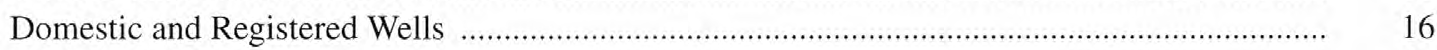

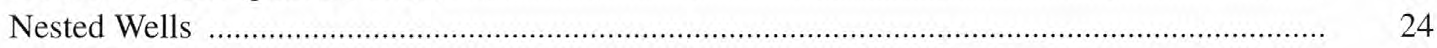

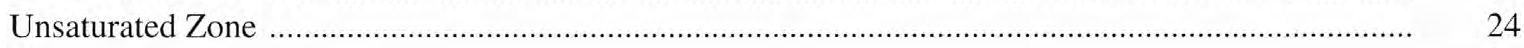

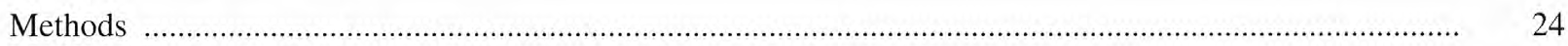

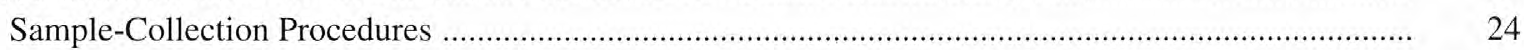

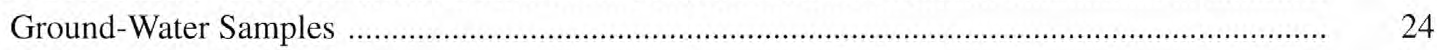

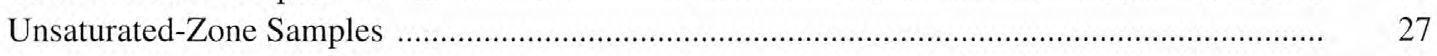

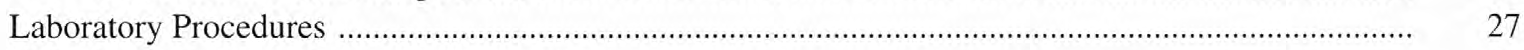

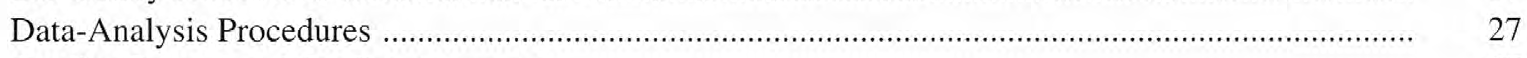

Quality Control and Quality Assurance ............................................................................................ 27

Trends in Nitrate Concentrations, Field Measurements, and Major-Ion and Tritium Concentrations

Spatial Trends in Nitrate Concentrations and Field Measurements in Ground Water ........................... 29

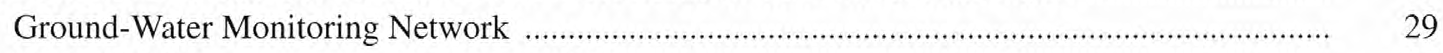

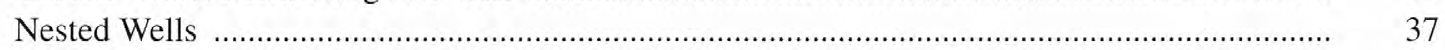

Spatial Trends in Major-Ion and Tritium Concentrations in Ground Water ....................................... 39

Temporal Trends in Nitrate Concentrations and Field Measurements in Ground Water ....................... 40

Spatial Trends in Nitrate Concentrations in Sediment of the Unsaturated Zone .............................................. 40

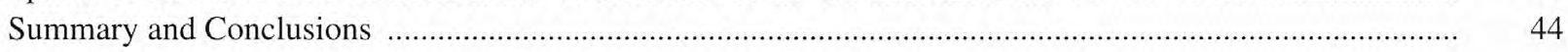

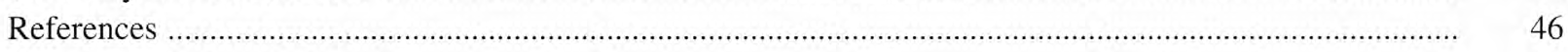

\section{FIGURES}

1-8. Maps showing:

1. Location of the Upper Big Blue Natural Resources District, central Nebraska........................... 3

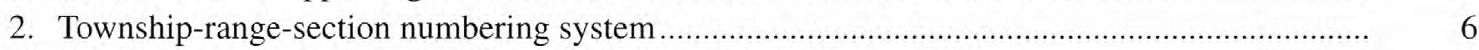

3. Locations of test holes and generalized geologic sections ..........................................................

4. Generalized geologic sections A-A' through E-E' showing the principal geologic units transected by test holes, Upper Big Blue Natural Resources District,

5. Thickness of High Plains aquifer and management zones, Upper Big Blue Natural Resources District, central Nebraska 3 6

6. Configuration of water-table altitude, Upper Big Blue Natural Resources District, central Nebraska, spring 1996 


\section{CONTENTS--Continued}

7. Ground-water-quality monitoring network indicating well depth, aquifer in which wells are completed, and locations of nested wells, Upper Big Blue River Natural

Resources District, central Nebraska

8. Nitrate concentrations and hydrogeologic conditions of the ground-water-quality monitoring network, Upper Big Blue Natural Resources District, central Nebraska, July 1995 through September 1996

9-12. Boxplots showing:

9. Distribution of nitrate concentrations by aquifer in the ground-waterquality monitoring network, Upper Big Blue Natural Resources District, central Nebraska, July 1995 through September 1996

10. Distribution of nitrate concentrations by hydrogeologic condition in the ground-water-quality monitoring network, Upper Big Blue Natural Resources

District, central Nebraska, July 1995 through September 1996

11. Distribution of nitrate concentrations by management zone in the groundwater-quality monitoring network, Upper Big Blue Natural Resources District central Nebraska, July 1995 through September 1996

12. Distribution of nitrate concentrations by well depth in the ground-waterquality monitoring network, Upper Big Blue Natural Resources District, central Nebraska, July 1995 through September 1996

13-16. Graphs showing

13. Trends in nitrate concentrations in water samples from nested wells, Upper Big Blue Natural Resources District, central Nebraska, November 1995 through May 1997

14. Nitrate concentrations in unsaturated-zone sediment samples from November and December 1995 and in water samples from nested wells from November 1995, by depth, Upper Big Blue Natural Resources District, central Nebraska

15. Nitrate concentrations in unsaturated-zone sediment samples from December 1996 and January 1997, and in water samples from nested wells from November 1996, by depth, Upper Big Blue Natural Resources District, central Nebraska

16. Nitrate concentrations in unsaturated-zone sediment samples from March 1997, and in water samples from nested wells from March 1997, by depth, Upper Big Blue Natural Resources District, central Nebraska.

\section{TABLES}

1. Summary of land use by county, Upper Big Blue Natural Resources District, central Nebraska, 1983-84

2. Generalized summary of the geologic units of Cretaceous age or younger and their waterbearing properties, Upper Big Blue Natural Resources District, central Nebraska .....

3. Test-hole sites used in generalized geologic sections, Upper Big Blue Natural Resources District, central Nebraska

4. Well information for ground-water-quality monitoring network of domestic and registered wells, Upper Big Blue Natural Resources District, central Nebraska

5. Locations and descriptions of nested wells, Upper Big Blue Natural Resources District, central Nebraska

6. Locations and descriptions of unsaturated-zone sampling subsites, Upper Big Blue Natural Resources District, central Nebraska

7. Results of split-sample analysis by the USGS National Water Quality Laboratory and the Grand Island Hall County Department of Health Laboratory..... 
8. Nitrate concentrations and field measurements for water samples from the ground-waterquality monitoring network of domestic and registered wells, Upper Big Blue Natural Resources District, central Nebraska, July 1995 through September 1997

9. Statistical summary of nitrate concentrations, field measurements, and well depth by aquifer, hydrogeologic condition, and management zone from the ground-water-quality monitoring network of domestic and registered wells, Upper Big Blue Natural Resources District, central Nebraska, July 1995 through September 1996

10. Results of Tukey's test for differences in nitrate concentrations among aquifers, Upper Big Blue Natural Resources District, central Nebraska

11. Results of Tukey's test for differences in nitrate concentrations among management zones, Upper Big Blue Natural Resources District, central Nebraska

12. Results of Tukey's test for differences in nitrate concentrations among well depths, Upper Big Blue Natural Resources District, central Nebraska

13. Nitrate concentrations and field measurements in water from nested monitoring wells, Upper Big Blue Natural Resources District, central Nebraska, July 1995 through May 1997

14. Summary statistics for nitrate concentrations from nested monitoring wells, Upper Big Blue Natural Resources District, central Nebraska, November 1995 through May 1997

15. Alkalinity, and major-ion and tritium concentrations in water samples from nested monitoring wells, Upper Big Blue Natural Resources District, central Nebraska, September 1996

16. Nitrate concentrations in unsaturated-zone sediment samples collected from selected subsites, Upper Big Blue Natural Resources District, central Nebraska, November 1995 through March 1997

\section{CONVERSION FACTORS AND VERTICAL DATUM}

\begin{tabular}{rcl}
\hline Multiply & By & To obtain \\
foot $(\mathrm{ft})$ & 0.3048 & meter \\
inch (in.) & 25.4 & millimeter \\
mile $(\mathrm{mi})$ & 1.609 & kilometer \\
square mile $\left(\mathrm{mi}^{2}\right)$ & 2.590 & square kilometer \\
acre & 4,047 & square meter \\
pound $(\mathrm{lb})$ & 0.4536 & kilogram \\
acre-foot $(\mathrm{acre}-\mathrm{ft})$ & 1.233 & cubic meter \\
foot per day $(\mathrm{ft} / \mathrm{d})$ & 0.3048 & meter per day \\
foot squared per day $(\mathrm{ft} / \mathrm{d})$ & 0.0929 & meter squared per day \\
inch per year $(\mathrm{in} / \mathrm{yr})$ & 25.4 & millimeter per year \\
foot per mile $(\mathrm{ft} / \mathrm{mi})$ & 0.1894 & meter per kilometer \\
gallon per day $(\mathrm{gal} / \mathrm{d})$ & 3.785 & liters per day \\
gallon per minute $(\mathrm{gal} / \mathrm{min})$ & 3.785 & liter per minute
\end{tabular}

Sea level: In this report, "sea level" refers to the National Geodetic Vertical Datum of 1929 (NGVD of 1929) - a geodetic datum derived from a general adjustment of the first-order level nets of both the United States and Canada, formerly called Sea Level Datum of 1929. 



\title{
Hydrogeology and Subsurface Nitrate in the Upper Big Blue Natural Resources District, Central Nebraska, July 1995 Through September 1997
}

\author{
By I.M. Verstraeten,V.L. McGuire, and K.L. Heckman
}

\section{ABSTRACT}

Following the designation of the Upper Big Blue Natural Resources District as a Special Protection Area by the Nebraska Department of Environmental Quality in 1993, the Upper Big Blue Natural Resources District requested that the U.S. Geological Survey, in cooperation with the Natural Resources District, develop a monitoring approach to identify the spatial and temporal variability of nitrate concentrations in ground water and assess the presence of nitrate concentrations in the unsaturated zone. A ground-water-quality monitoring network of 197 existing domestic and registered wells was proposed, along with 8 nested wells (18 wells) and associated unsaturated-zone subsites. Most of the wells are completed in the shallow (104), deep (86), and combined (3) parts of the High Plains aquifer, with 123 wells screened in the unconfined parts and 70 wells screened in the confined part. One well is completed in the Greenhorn Formation, and three wells are completed in the Dakota aquifer.

The ground water in the Upper Big Blue Natural Resources District had a median nitrate concentration of $4.6 \mathrm{mg} / \mathrm{L}$ (milligrams per liter). Water samples from the shallow and deep parts of the High Plains aquifer had median nitrate concentrations of 6.3 and $3.4 \mathrm{mg} / \mathrm{L}$, respectively. Water from the Dakota aquifer had a median nitrate concentration of $0.11 \mathrm{mg} / \mathrm{L}$. Forty-one of the 197 samples (21 percent) exceeded the U.S. Environmental Protection Agency Maximum Contaminant Level for drinking water of $10 \mathrm{mg} / \mathrm{L}$ for nitrate as nitrogen. Among the 12 management zones established by the Upper Big Blue Natural
Resources District to manage water quantity and quality, the median nitrate concentrations varied from $0.97 \mathrm{mg} / \mathrm{L}$ in zone 12 to $10 \mathrm{mg} / \mathrm{L}$ in zone 5 . The latter median concentration exceeded the Phase II trigger of $9 \mathrm{mg} / \mathrm{L}$ established by the Upper Big Blue Natural Resources District in their Special Protection Area Action Plan.

Nitrate concentrations and field measurements of water samples collected from the nested wells generally agree with the results from the proposed ground-water-quality monitoring network of domestic and registered wells. Nitrate concentrations in water samples from the well nests generally did not appear to vary with time during the 19 months of data collection. Major-ion data indicate that water in the High Plains aquifer generally is a calcium-bicarbonate type and shows little spatial variation. The tritium data indicate that water from the shallow part of the High Plains aquifer likely was recharged during the last 10 to 20 years, while water from the deep, confined part of the High Plains aquifer likely was recharged more than 50 years ago. The tritium data indicate that, where confined conditions exist in the deep part of the High Plains aquifer and have not been modified through penetration of wells, the water could remain unaffected by nitrate contamination.

Nitrate concentrations of 245 unsaturatedzone sediment samples analyzed varied from less than 0.05 to 69 milligrams per kilogram with a median of 3.7 milligrams per kilogram. Nitrate concentrations in the unsaturated-zone sediment samples varied among sample subsites, and were not related to the depth at which the samples were collected. At site DH4, nitrate concentrations in 
unsaturated-zone sediment samples collected below nonirrigated cropland were larger than nitrate concentrations from samples collected below irrigated or pasture land. Nitrate concentrations below pasture land generally were smaller than nitrate concentrations below cropland.

\section{INTRODUCTION}

The Nebraska Department of Environmental Quality (NDEQ) conducted a water-quality study in the Upper Big Blue Natural Resources District (UBBNRD) (fig. 1) in central Nebraska from 1989 through 1991 to determine if the ground water was sufficiently contaminated to require Special Protection Area designation (Gottula, 1990; Gottula and Link, 1992). Results from a water-quality monitoring program of the UBBNRD conducted prior to the NDEQ study indicated that nitrate concentrations in the principal aquifers were increasing (R.D. DeBuhr, UBBNRD, written commun., 1997). In addition, the City of Seward, whose municipal water supply contains large nitrate concentrations, requested further investigation. On September 23, 1993, the NDEQ declared the UBBNRD a Special Protection Area. Twelve management zones, and action levels at the Phase II trigger of $9 \mathrm{mg} / \mathrm{L}$ (milligrams per liter) and Phase III trigger of $12 \mathrm{mg} / \mathrm{L}$, were established in the UBBNRD's Special Protection Area Action Plan, potentially imposing increased monitoring or best management practices (BMPs) in the affected management zones (Upper Big Blue Natural Resources District, 1994).

Following the designation of the Special Protection Area, the UBBNRD formed a citizens advisory committee and requested that the U.S. Geological Survey (USGS) develop a monitoring approach that with time: (1) would identify the spatial and temporal variability of nitrate concentrations in ground water with emphasis on the shallow part of the High Plains aquifer (the major source of water underlying the UBBNRD); and (2) would quantify nitrate concentrations in the unsaturated zone to aid in developing and assessing long-term BMPs. The UBBNRD received a Clean Water Act 319 grant (RFP No. SWSRF94-1) for the UBBNRD's Special Protection Area Groundwater Quality Program used in part to finance the installation of nested wells discussed herein.
Previous studies have determined that the general water quality of the High Plains aquifer in the study area is a calcium-bicarbonate type (Johnson and Keech, 1959; Keech and Dreeszen, 1959; Keech, 1962 and 1978; Gottula, 1990; Gottula and Link, 1992). A regional study of nitrates by Kolpin and others (1994) demonstrated that large nitrate concentrations were detected in the nearsurface unconsolidated aquifer in the study area. The UBBNRD, through the ground-water-quality program started in the 1980 s, identified an increase in nitrate concentrations from the early to late $1980 \mathrm{~s}$ (Gottula and Link, 1992). Deep core samples were collected by the UBBNRD in 1990 and analyzed for nitrogen species (Gottula and Link, 1992). Results showed potential movement of nitrogen below the root zone and generally larger nitrogen levels under cropland than under pasture land.

The USGS, in cooperation with the UBBNRD, (1) conducted a hydrogeologic study, (2) evaluated the UBBNRD's existing ground-water-quality monitoring network, and (3) developed a saturatedand unsaturated-zone monitoring approach in the entire UBBNRD from 1995 through 1997 based on land use, physiography, soil, geology, and hydrology. The objectives of the study were to: (1) describe the hydrogeology of the study area (the UBBNRD); (2) develop a monitoring approach to evaluate the spatial and temporal variations in subsurface nitrate concentrations, including unsaturated zone and principal aquifers; and (3) perform an initial assessment of nitrate and major-ion concentrations in ground water and nitrate in the unsaturated zone in the study area. The intent of this study was to aid the UBBNRD with the development of data collection efforts for determining long-term effectiveness of current and future management practices that may be beneficial in the reduction of nitrate in the ground water of the study area. Results of this study also may be useful in addressing these issues in other areas with similar hydrogeologic settings. 


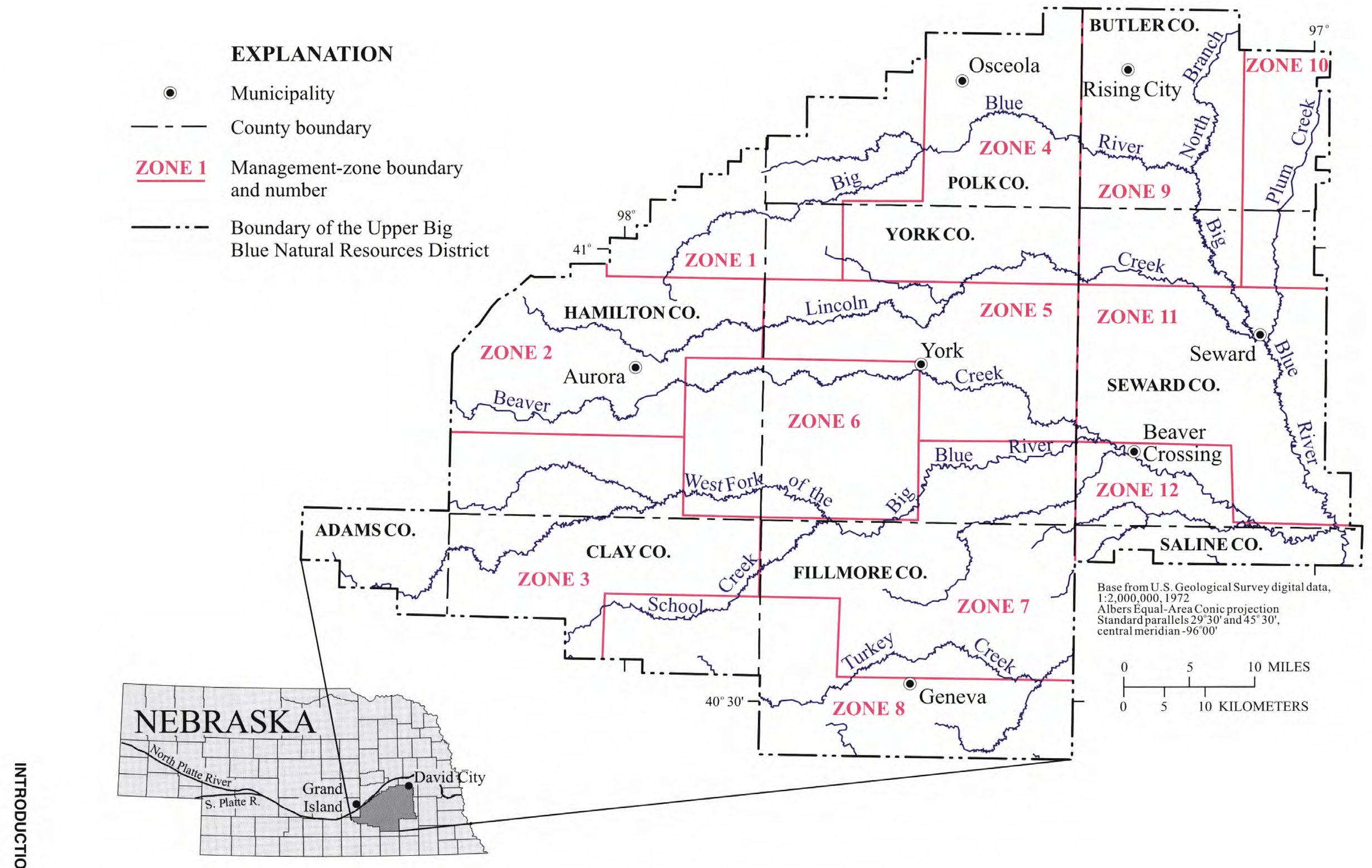

Figure 1. Location of the Upper Big Blue Natural Resources District, central Nebraska. 


\section{Purpose and Scope}

This report presents the results of the study. The monitoring approach used about 200 domestic and registered irrigation, municipal, and industrial wells, along with 18 nested wells at 8 nested well sites, and associated unsaturated-zone subsites, established for this study. Spatial and temporal trends in nitrate concentrations in ground water and the unsaturated zone are described. Spatial trends of major-ion concentrations, and results of analyses of tritium concentrations used for estimating the relative age of water, also are described. Hydrogeologic data used in this study were collected from July 1995 through September 1997.

\section{Description of Study Area}

The study area comprises about $2,860 \mathrm{mi}^{2}$. According to the 1990 census, the population in the study area was 55,264 , of which 66 percent was urban. The study area mainly lies in the upper reaches of the Big Blue River Basin in central Nebraska (fig. 1) and consists mostly of a gently rolling loess (wind-deposited silt) upland of low relief dissected by small meandering streams occupying wide shallow valleys (Johnson and Keech, 1959). The primary streams in the study area are the Big Blue River and its tributaries, Turkey Creek, School Creek, West Fork of the Big Blue River, Beaver Creek, Lincoln Creek, North Branch of the Big Blue River, and Plum Creek. Generally, these streams flow to the east southeast (fig. 1). Small shallow marshes mainly are in Clay County.

About 85 percent of the land is upland, 10 percent is terrace land, and 3 percent is foot slopes - the area between upland and bottomland. Generally, the parent material of the soil is loess (about 90 percent), especially on the upland. Only 1 percent of the parent material is glacial till and about 1 percent is alluvium (unpublished data from U.S. Natural Resources Conservation Service, written commun., 1983). Areas with soils having glacial till as parent material mainly are in Butler and Seward Counties in the eastern part of the study area (fig. 1).

In part, the susceptibility of the land to movement of contaminants through the unsaturated zone is reflected by the drainage class of the soil, which in turn is affected by the parent material of the soil. Approximately 88 percent of the land has soils that are well drained and 10 percent of the land has soils that are poorly drained (unpublished data from U.S. Natural Resources Conservation Service, written commun., 1983). As a result, ground water in the area generally may be susceptible to nonpointsource contamination. The poorly-drained soils generally are on the upland and have B horizons - a layer within the soil generally consisting of illuvial colloids located below the A horizon which itself tends to be darkened by an accumulation of organic matter. In the marshy areas and in areas with glacial till as parent material, the soils tend to be poorly drained. Only about 1 percent of the land is excessively drained. These areas generally are on the bottomlands (terraces and floodplains) near the streams.

The climate is typical of a mid-latitude, continental setting with pronounced seasonal ranges in temperature. The mean annual precipitation in the area ranges from about 25 in. in the western part of the study area to $27 \mathrm{in}$. in the eastern part. Most precipitation is received during thunderstorms in the spring and summer.

The study area is one of the more intensively cultivated and irrigated areas in Nebraska. About 54 percent of all the land in the study area is irrigated cropland (table 1). About 33 percent of the land is dry cropland and about 9 percent of the land is dry pasture and range land. Other land uses such as urban areas, forest land and surface water make up less than 4 percent of the land mass. These statistics are based on land use in the part of each county in the study area (unpublished data from U.S. Natural Resources Conservation Service, written commun., 1983).

\section{Acknowledgments}

The authors thank the well owners in the study area for granting permission to sample their wells and for their assistance in the field. The authors thank Vince Dreeszen (formerly with the Conservation and Survey Division of Nebraska) for his comments and input on a portion of the description of the hydrogeology of the study area. 
Table 1. Summary of land use by county, Upper Big Blue Natural Resources District, central Nebraska, 1983-84

[all units are in square miles; unpublished data from U.S. Natural Resources Conservation Service, written commun., 1983]

\begin{tabular}{lcrrrrrr}
\hline \multicolumn{7}{c}{ County } & \multicolumn{7}{c}{ Land-use class } \\
\cline { 2 - 8 } \multicolumn{1}{c}{} & $\begin{array}{c}\text { Irrigated } \\
\text { cropland }\end{array}$ & $\begin{array}{c}\text { Dry crop- } \\
\text { land }\end{array}$ & $\begin{array}{c}\text { Irrigated } \\
\text { pasture } \\
\text { land }\end{array}$ & $\begin{array}{c}\text { Dry } \\
\text { pasture } \\
\text { and range } \\
\text { land }\end{array}$ & Forest land & $\begin{array}{c}\text { Surface } \\
\text { water }\end{array}$ & Other \\
\hline Adams & 43 & 21 & 0.1 & 3.8 & 0.0 & 0.3 & 3.2 \\
Butler & 81 & 150 & .2 & 23 & .0 & .3 & 5.9 \\
Clay & 141 & 73 & .2 & 23 & 1.4 & .3 & 5.2 \\
Fillmore & 213 & 159 & .3 & 44 & .6 & .9 & 15 \\
Hamilton & 355 & 83 & .4 & 39 & .8 & .7 & 21 \\
Polk & 139 & 89 & .0 & 17 & .0 & .5 & 11 \\
Saline & 31 & 23 & .0 & 7.2 & .0 & .1 & 2.4 \\
Seward & 157 & 245 & .0 & 41 & 6.9 & .2 & 9.7 \\
York & 387 & 107 & .0 & 57 & .0 & .8 & 24 \\
Total & 1.547 & 950 & 1.2 & 255 & 9.7 & 4.1 & 97.4 \\
\hline
\end{tabular}

\section{HYDROGEOLOGY}

The description of the hydrogeology of the study area is based on hydrologic and geologic information contained in published and unpublished reports by the Conservation and Survey Division (CSD), Institute of Agriculture and Natural Resources, University of Nebraska-Lincoln and by the USGS. A township-range-section system (fig. 2) was used in the report to identify well and site locations. A summary of the geologic units that comprise the unconsolidated and bedrock deposits beneath the study area and corresponding hydrogeologic units and water-bearing properties (table 2) was made. Test holes (fig. 3, table 3) provided data used in construction of generalized geologic sections (fig. 4). Previous studies were used in summarizing the hydrogeology of the study area including: Ellis (1986); Emery (1966); Ginsberg (1983); Johnson and Keech (1959); Keech (1962 and 1978); Keech and Dreeszen, (1959, 1968a, and 1968b); Keech, and others (1967); Pettijohn and Chen (1983a); Gottula (1990); and Gottula and Link (1992).
Water-Bearing Hydrogeologic Units

\section{Unconsolidated Deposits}

The High Plains aquifer is the principal source of water in the study area. This aquifer consists of alluvial clay, silt, sand, and gravel and in some areas glacial till of Quaternary age and alluvial clayey and sandy silt of Tertiary age (table 2). For purposes of this report the High Plains aquifer has been subdivided into a shallow part (mainly consisting of sediments of Illinoian age) and a deep part (mainly consisting of sediments of pre-Illinoian age at times separated by glacial till). The hydrogeologic conditions of aquifer parts are designated as unconfined (UF) and confined (CF).

The hydrologic conditions of the High Plains aquifer vary from unconfined, perched to confined conditions. The base of the High Plains aquifer is the bottom of the lowermost sand and gravel units, generally corresponding with the top of the bedrock, including the Ogallala Formation. The thickness of the High Plains aquifer (fig. 5) is in part controlled by the relief of the bedrock surface. Generally, the 


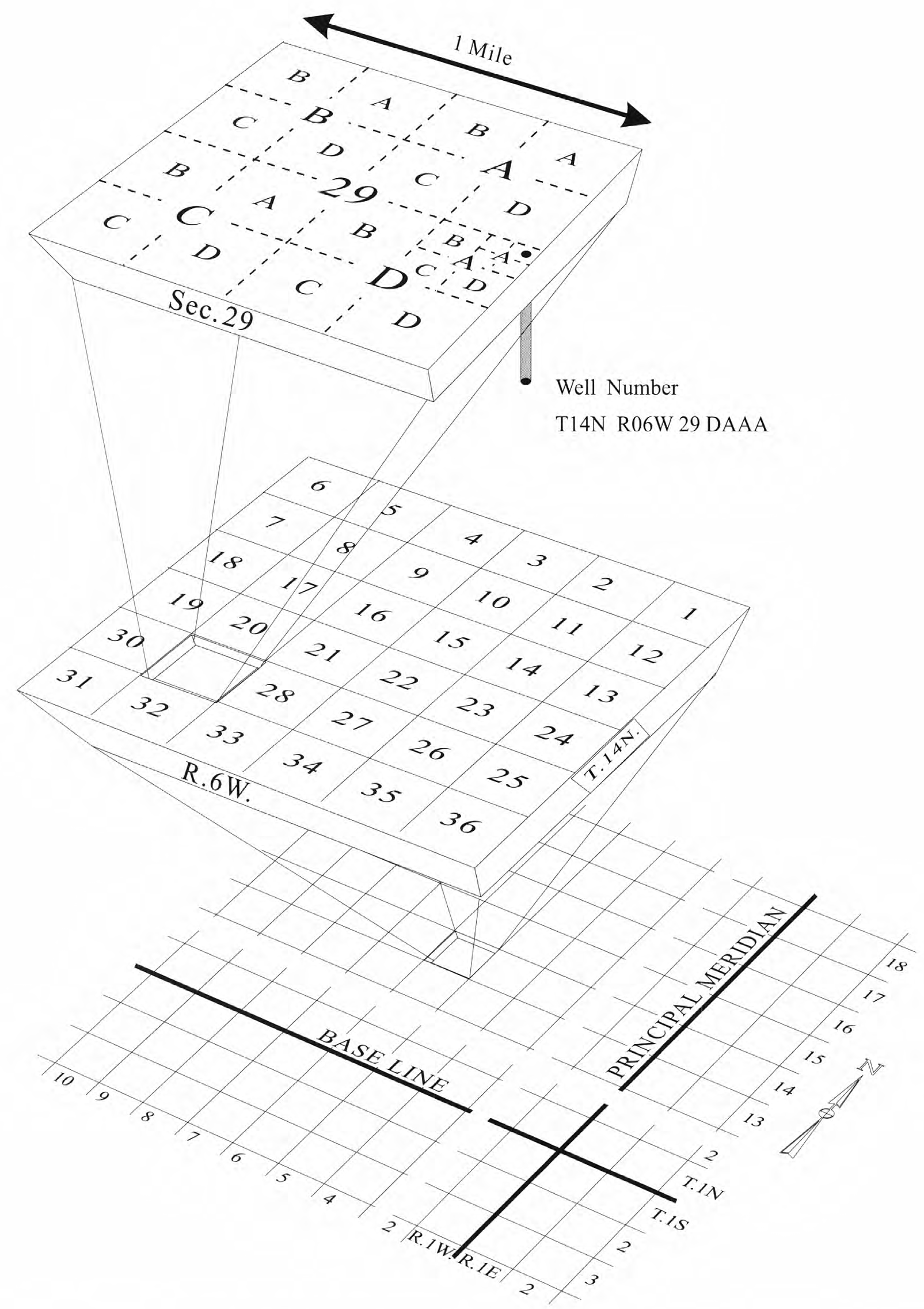

Figure 2. Township-range-section numbering system. 
Table 2. Generalized summary of the geologic units of Cretaceous age or younger and their water-bearing properties, Upper Big Blue Natural Resources District, central Nebraska

$[--$, water-bearing units absent]

\begin{tabular}{|c|c|c|c|c|c|c|}
\hline System & Series & Geologic unit & $\begin{array}{l}\text { Approxi- } \\
\text { mate thick- } \\
\text { ness (feet) }\end{array}$ & Description & $\begin{array}{l}\text { Water-bearing hydro- } \\
\text { geologic unit }\end{array}$ & Water-bearing properties \\
\hline \multirow[t]{2}{*}{ Quaternary } & $\begin{array}{l}\text { Holocene } \\
\quad \text { (Recent) }\end{array}$ & $\begin{array}{l}\text { Undifferentiated } \\
\text { soil, surficial } \\
\text { alluvium, and } \\
\text { loess deposits }\end{array}$ & $0-17$ & $\begin{array}{l}\text { Widespread soil; alluvial deposits of } \\
\text { clay, silt, sand, and gravel; isolated } \\
\text { wind-deposited silt and clay. }\end{array}$ & -- & $\begin{array}{l}\text { Not a known source of water supply because } \\
\text { deposits generally are unsaturated; significant } \\
\text { as a permeable conduit for ground-water } \\
\text { recharge. }\end{array}$ \\
\hline & Pleistocene & $\begin{array}{l}\text { Undifferentiated } \\
\text { unconsolidated } \\
\text { deposits }\end{array}$ & $0-460$ & $\begin{array}{l}\text { Wind-deposited silt and clay; terrace } \\
\text { deposits of sand and gravel; paleosols; } \\
\text { unsorted and unstratified glacial till; } \\
\text { alluvial sand, silt, and gravel of Illi- } \\
\text { noian, and pre-Illinoian age; silt with a } \\
\text { basal gravel locally. Underlies most of } \\
\text { the study area. }\end{array}$ & $\begin{array}{l}\text { High Plains aquifer and } \\
\text { undifferentiated } \\
\text { glacial till aquifer }\end{array}$ & $\begin{array}{l}\text { Sand and gravel can yield abundant water to } \\
\text { wells if water is present; these deposits are the } \\
\text { principal source of ground water in the study } \\
\text { area and comprise a major part of the High } \\
\text { Plains aquifer; water-bearing units are uncon- } \\
\text { fined, perched, semiconfined, or confined in } \\
\text { the study area. Sand lenses in glacial till, not } \\
\text { hydraulically connected to the main aquifer, } \\
\text { can yield small amounts of water to wells in } \\
\text { the eastern part of the study area near Seward. }\end{array}$ \\
\hline \multirow[t]{2}{*}{ Tertiary } & Pliocene & $\begin{array}{l}\text { Undifferentiated } \\
\text { unconsolidated } \\
\text { silt }\end{array}$ & $0-130$ & Silt that mainly blankets bedrock. & -- & $\begin{array}{l}\text { Not a known source of water supply; generally } \\
\text { too fine textured to yield water to wells. }\end{array}$ \\
\hline & Miocene & $\begin{array}{l}\text { Ogallala } \\
\text { Formation }\end{array}$ & $0-130$ & $\begin{array}{l}\text { Alluvial sandy and clayey brownish gray } \\
\text { and gray silt; partially calcareous and } \\
\text { mostly unconsolidated; locally con- } \\
\text { tains lenses of sand and basal gravel; } \\
\text { underlies a small area in the western } \\
\text { part of the study area. }\end{array}$ & High Plains aquifer & $\begin{array}{l}\text { Generally a minor source of water supply in the } \\
\text { study area; may yield sufficient amounts of } \\
\text { water to domestic wells; constitutes a small } \\
\text { part of the High Plains aquifer in the study } \\
\text { area. }\end{array}$ \\
\hline \multirow[t]{4}{*}{ Cretaceous } & $\begin{array}{l}\text { Upper } \\
\text { Cretaceous }\end{array}$ & $\begin{array}{l}\text { Niobrara } \\
\text { Formation }\end{array}$ & $0-260$ & $\begin{array}{l}\text { Yellow and gray marine chalky shale and } \\
\text { chalk; underlying most of the study } \\
\text { area. }\end{array}$ & $\begin{array}{l}\text { Niobrara water-bearing } \\
\text { units }\end{array}$ & $\begin{array}{l}\text { Generally not a known source of water supply; } \\
\text { can yield water to wells where fractured in } \\
\text { Fillmore County. }\end{array}$ \\
\hline & & Carlile Shale & $0-230$ & $\begin{array}{l}\text { Gray marine shale; calcareous and } \\
\text { fossiliferous in the lower part; } \\
\text { underlying most of the study area. }\end{array}$ & -- & Not a known source of water supply. \\
\hline & & $\begin{array}{l}\text { Greenhorn } \\
\text { Limestone }\end{array}$ & $0-50$ & $\begin{array}{l}\text { Gray fossiliferous limestone interbedded } \\
\text { with calcareous shale; underlying most } \\
\text { of the study area. }\end{array}$ & -- & Not a known source of water supply. \\
\hline & $\begin{array}{l}\text { Lower } \\
\text { Cretaceous }\end{array}$ & Dakota Group & $240-680$ & $\begin{array}{l}\text { Interbedded clay, silty and sandy shale, } \\
\text { and sandstone; underlies the entire } \\
\text { study area. }\end{array}$ & Dakota aquifer & $\begin{array}{l}\text { Contains moderately to highly mineralized } \\
\text { water; sandstone layers comprise the Dakota } \\
\text { aquifer and yield water to wells; deep wells in } \\
\text { the eastern part of the study area obtain water } \\
\text { from this aquifer. }\end{array}$ \\
\hline
\end{tabular}




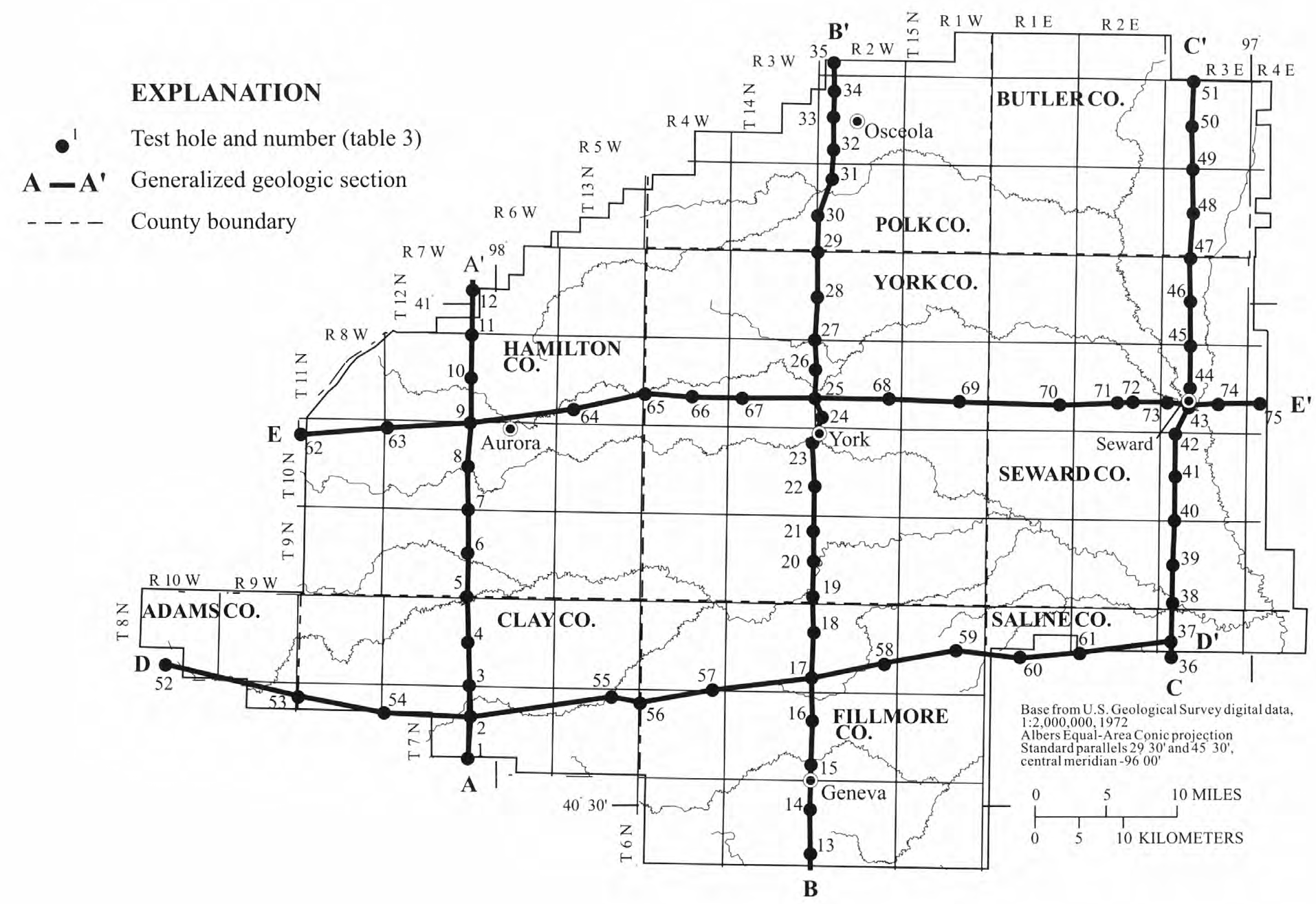

Figure 3. Locations of test holes and generalized geologic sections (figure 4). 

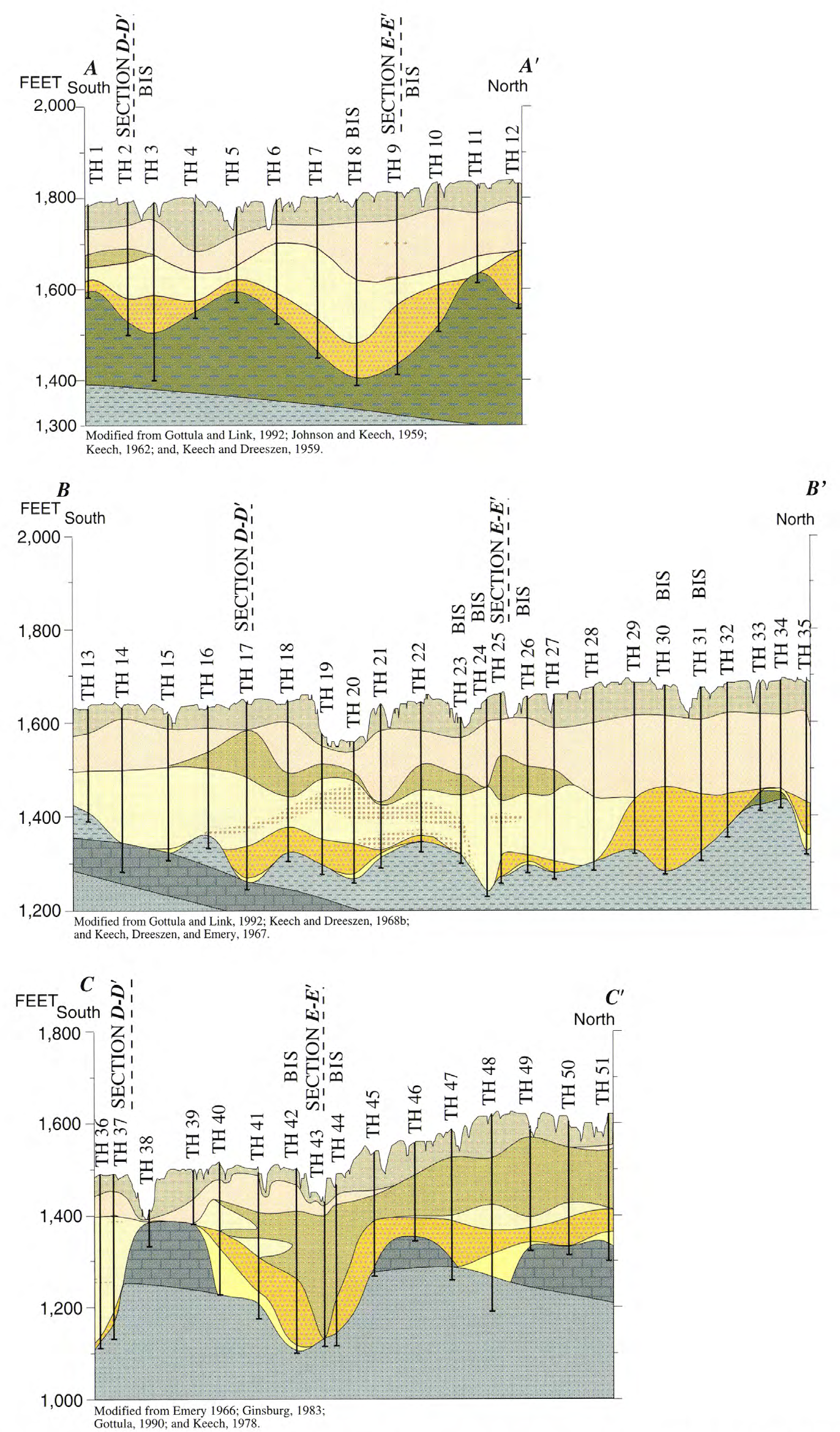
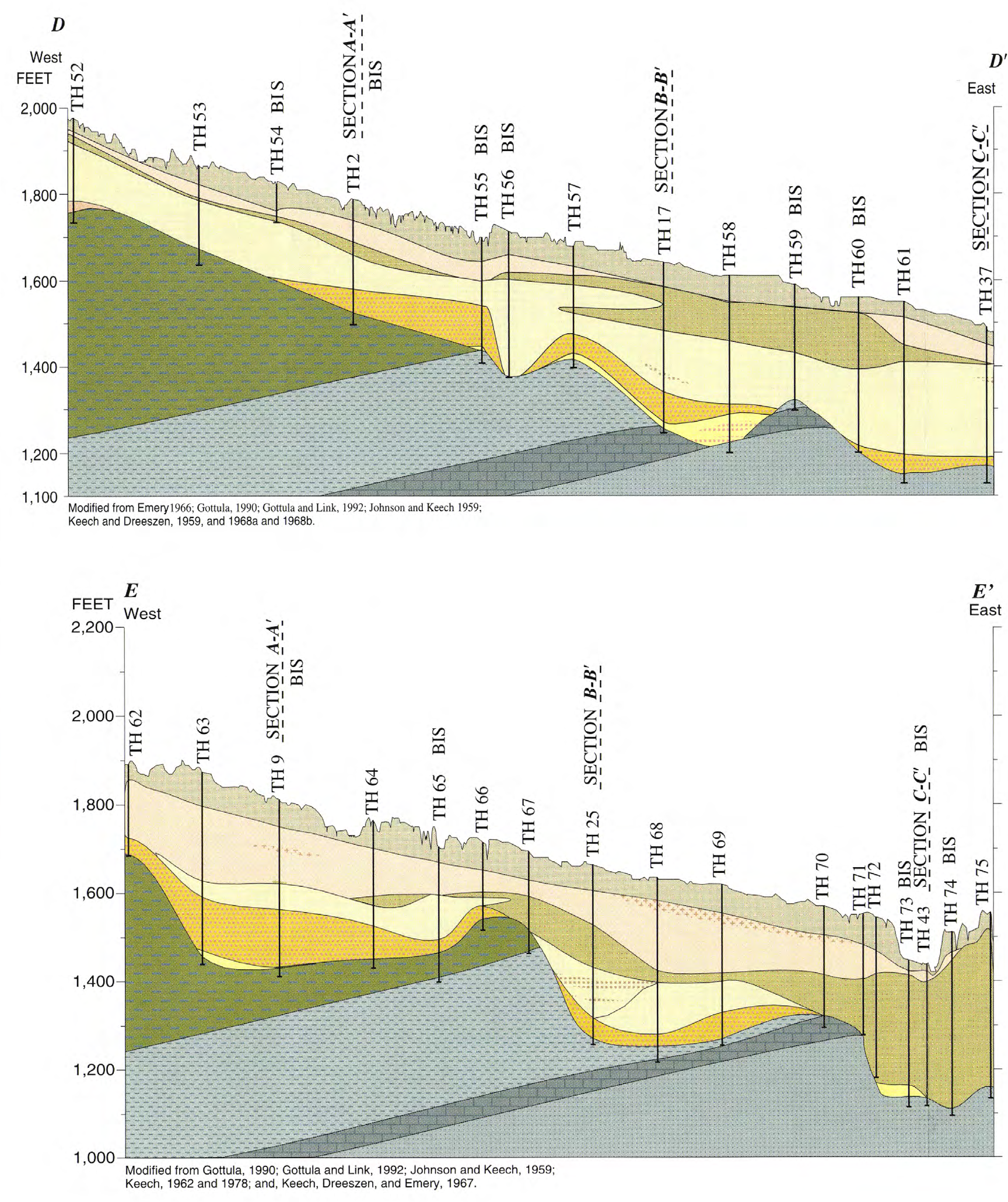

East Tertiary

\section{EXPLANATION}

System Geologic unit and character

$\square$ Soil, surficial

$\square \begin{aligned} & \text { Soil, surficial } \\ & \text { alluvium, and loess }\end{aligned}$

Upper sand and

gravel, stippled pattern indicates silt and clay lenses with some sand

Clay, silt and
sand or glacial till

Quaternary-

Lower sand and gravel,
stippled pattern indicate stippled pattern indicates some sand or glacial till

Silt and clay with

Sand and gravel, stippled clay lenses

Silt, clay, and gravel

of Ogallala Formation

Shale and chalk
of Niobrara Formatio of Niobrara For

Carlile Shale

Greenhorn Limestone and Graneros Shale

Dakota Group,

interbedded with clay

$\stackrel{n}{2}$

Test hole and number

BIS Bend in section

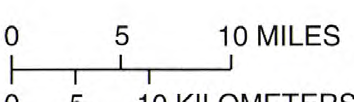

VERTICAL SCALE

GREATLY EXAGGERATED

DATUM IS SEA LEVEL

HYDROGEOLOGY 
Table 3. Test-hole sites used in generalized geologic sections, Upper Big Blue Natural Resources District, central Nebraska

\begin{tabular}{|c|c|c|c|}
\hline $\begin{array}{l}\text { Test-hole site } \\
\text { (fig. 3) }\end{array}$ & Geologic section & $\begin{array}{l}\text { Conservation and Survey Division } \\
\text { test-hole identification number }\end{array}$ & $\begin{array}{l}\text { Location } \\
\text { (fig. 2) }\end{array}$ \\
\hline 1 & $A-A^{\prime}$ & $34-B-49$ & T07N R07W 25 DD \\
\hline 2 & $\mathrm{~A}-\mathrm{A}^{\prime}$ and $\mathrm{D}-\mathrm{D}^{\prime}$ & 40-U-41 & T07N R06W 18 BB \\
\hline 3 & $A-A^{\prime}$ & $35-B-49$ & T08N R07W 36 DD \\
\hline 4 & $A-A^{\prime}$ & $2-40$ & T08N R07W 24 AA \\
\hline 5 & A- $A^{\prime}$ & $36-B-49$ & T09N R07W 36 DD \\
\hline 6 & $A-A^{\prime}$ & $37-B-49$ & T09N R07W 13 DD \\
\hline 7 & $A-A^{\prime}$ & $38-B-49$ & T10N R07W 36 DD \\
\hline 8 & $A-A^{\prime}$ & $39-B-49$ & T10N R07W 24 AA \\
\hline 9 & $\mathrm{~A}-\mathrm{A}^{\prime}$ and $\mathrm{E}-\mathrm{E}^{\prime}$ & $40-B-49$ & T10N R07W $01 \mathrm{AA}$ \\
\hline 10 & A-A' & $41-B-49$ & T11N R07W 13 DD \\
\hline 11 & $A-A^{\prime}$ & $42-B-49$ & T11N R07W $01 \mathrm{AA}$ \\
\hline 12 & $A-A^{\prime}$ & $43-B-49$ & T12N R07W 13 DD \\
\hline 13 & B-B' & $58-\mathrm{A}-49$ & T06N R02W 31 BB \\
\hline 14 & B-B' & $59-A-49$ & T06N R03W 13 AA \\
\hline 15 & B-B' & $64-B-49$ & T07N R03W 25 DD \\
\hline 16 & B-B' & $63-B-49$ & T07N R02W 07 CC \\
\hline 17 & B-B' and D-D' & $39-\mathrm{U}-41$ & T08N R03W 36 AA \\
\hline 18 & B-B' & $62-B-49$ & T08N R02W 07 CC \\
\hline 19 & B-B' & $61-B-49$ & T09N R02W 31 BC \\
\hline 20 & B-B' & $60-B-49$ & T09N R02W 19 BB \\
\hline 21 & B-B' & $59-B-49$ & T09N R03W 12 AA \\
\hline 22 & B-B' & $58-B-49$ & T10N R02W 19 CC \\
\hline 23 & B-B' & $57-B-49$ & T10N R03W 01 DD \\
\hline 24 & B-B' & $58-\mathrm{A}-57$ & T11N R02W $31 \mathrm{BA}$ \\
\hline 25 & $B-B^{\prime}$ and $E-E^{\prime}$ & $46-B-46$ & T11N R03W 24 DD \\
\hline 26 & B-B' & $56-B-49$ & T11N R03W 12 DD \\
\hline 27 & B-B' & $55-\mathrm{B}-49$ & T12N R03W 36 DD \\
\hline 28 & B-B' & 54-B-49 & T12N R02W $19 \mathrm{BB}$ \\
\hline 29 & B-B' & $53-B-49$ & T13N R02W $31 \mathrm{CC}$ \\
\hline 30 & B-B' & $52-B-49$ & T13N R03W $24 \mathrm{AD}$ \\
\hline 31 & B-B' & 51-B-49 & T13N R02W 06 DD \\
\hline 32 & B-B' & $17-B-45$ & T14N R02W 29 CC \\
\hline 33 & B-B' & $50-B-49$ & T14N R02W 18 DA \\
\hline 34 & B-B' & $18-B-45$ & T14N R02W $05 \mathrm{CC}$ \\
\hline 35 & B-B' & $49-B-49$ & T15N R02W $31 \mathrm{AA}$ \\
\hline 36 & $\mathrm{C}-\mathrm{C}^{\prime}$ & $45-A-59$ & T08N R03E 19 AD \\
\hline 37 & C-C' \& D-D' & $36-\mathrm{U}-41$ & T08N R03E $18 \mathrm{AA}$ \\
\hline 38 & $\mathrm{C}-\mathrm{C}^{\prime}$ & $26-B-48$ & T09N R03E 31AA \\
\hline 39 & $\mathrm{C}-\mathrm{C}^{\prime}$ & $18-A-48$ & T09N R03E $18 \mathrm{AA}$ \\
\hline 40 & $\mathrm{C}-\mathrm{C}^{\prime}$ & $17-\mathrm{A}-48$ & T10N R03E 32 CC \\
\hline 41 & $\mathrm{C}-\mathrm{C}^{\prime}$ & $16-\mathrm{A}-48$ & T10N R03E $17 \mathrm{CC}$ \\
\hline 42 & $\mathrm{C}^{-\mathrm{C}^{\prime}}$ & $15-A-48$ & T10N R03E 05 BB \\
\hline 43 & C-C' \& E-E' & $14-\mathrm{A}-48$ & T11N R03E $29 \mathrm{AA}$ \\
\hline
\end{tabular}


Table 3. Test-hole sites used in generalized geologic sections, Upper Big Blue Natural Resources District, central Nebraska --Continued

\begin{tabular}{|c|c|c|c|}
\hline $\begin{array}{l}\text { Test-hole site }^{1} \\
\text { (fig. 3) }\end{array}$ & Geologic section & $\begin{array}{l}\text { Conservation and Survey Division } \\
\text { test-hole identification number }\end{array}$ & $\begin{array}{l}\text { Location } \\
\text { (fig. 2) }\end{array}$ \\
\hline 44 & C-C' & $23-B-48$ & T11N R03E $16 \mathrm{BB}$ \\
\hline 45 & $\mathrm{C}-\mathrm{C}^{\prime}$ & $22-B-48$ & T11N R03E $04 \mathrm{BB}$ \\
\hline 46 & $\mathrm{C}-\mathrm{C}^{\prime}$ & $21-B-48$ & T12N R03E $17 \mathrm{CC}$ \\
\hline 47 & $\mathrm{C}-\mathrm{C}^{\prime}$ & $20-B-48$ & T12N R03E 05 BB \\
\hline 48 & $\mathrm{C}-\mathrm{C}^{\prime}$ & $19-B-48$ & T13N R03E 16 CC \\
\hline 49 & $\mathrm{C}^{-\mathrm{C}^{\prime}}$ & $18-B-48$ & T14N R03E 32 DD \\
\hline 50 & $\mathrm{C}-\mathrm{C}^{\prime}$ & $17-B-48$ & T14N R03E $20 \mathrm{AA}$ \\
\hline 51 & $\mathrm{C}-\mathrm{C}^{\prime}$ & $16-B-48$ & T15N R03E 32 DD \\
\hline 52 & D-D' & $13-B-46$ & T08N R10W 32 AA \\
\hline 53 & D-D' & $14-B-46$ & T07N R09W $12 \mathrm{AA}$ \\
\hline 54 & D-D' & $3-40$ & T07N R07W 07 CC \\
\hline 55 & D-D' & $38-\mathrm{U}-41$ & T07N R05W 03 DA \\
\hline 56 & D-D' & $4-A-59$ & T07N R05W 01 DD \\
\hline 57 & D-D' & $18-B-46$ & T08N R04W 36 CC \\
\hline 58 & D-D' & $30-B-45$ & T08N R02W 25 BB \\
\hline 59 & D-D' & $29-B-45$ & T08N R01W 22 AA \\
\hline 60 & $\mathrm{D}^{-\mathrm{D}^{\prime}}$ & $37-U-41$ & T08N R01E 21 AD \\
\hline 61 & $D^{-} D^{\prime}$ & $42-\mathrm{U}-41$ & T08N R02E $19 \mathrm{AB}$ \\
\hline 62 & E-E' & $112-B-47$ & T10N R09W $12 \mathrm{AA}$ \\
\hline 63 & E-E' & $45-B-49$ & T10N R07W 06 BC \\
\hline 64 & E-E' & $47-B-49$ & T11N R05W 29 CC \\
\hline 65 & E-E' & $40-B-46$ & T11N R04W 19 CC \\
\hline 66 & E-E' & $48-B-49$ & T11N R04W 22 CD \\
\hline 67 & E-E' & $45-B-46$ & T11N R03W $30 \mathrm{AA}$ \\
\hline 68 & E-E' & $47-B-46$ & T11N R02W 24 CC \\
\hline 69 & E-E' & $23-B-45$ & T11N R01W $27 \mathrm{AA}$ \\
\hline 70 & E-E' & $6-B-44$ & T11N R01E 26 AA \\
\hline 71 & E-E' & $2-A-58$ & T11N R02E 21 DD \\
\hline 72 & E-E' & $2-B-44$ & T11N R02E $23 \mathrm{CC}$ \\
\hline 73 & E-E' & $1-B-44$ & T11N R03E 19 CD \\
\hline 74 & E-E' & $13-\mathrm{A}-48$ & T11N R03E 22 DD \\
\hline 75 & $E^{\prime}-E^{\prime}$ & 41-U-41 & T11N R04E 19 DD \\
\hline
\end{tabular}

${ }^{1}$ Note: The Conservation and Survey Division of the University of Nebraska county test-hole log books references are: Adams County: Conservation and Survey Division, University of Nebraska, written commun., no date; Butler and Colfax County: Conservation and Survey Division. University of Nebraska, written commun., no date; Clay County: by Burchett and Smith, 1994; Fillmore County: by Conservation and Survey Division and U.S. Geological Survey, 1953; Hamilton County: by Keech, 1960; Polk County: by Burchett and Smith, 1996; Saline County: by Smith, Emery, and Souders, 1964; Seward County: by Burchett and Smith, 1993; and York County: by Smith, 1963. 


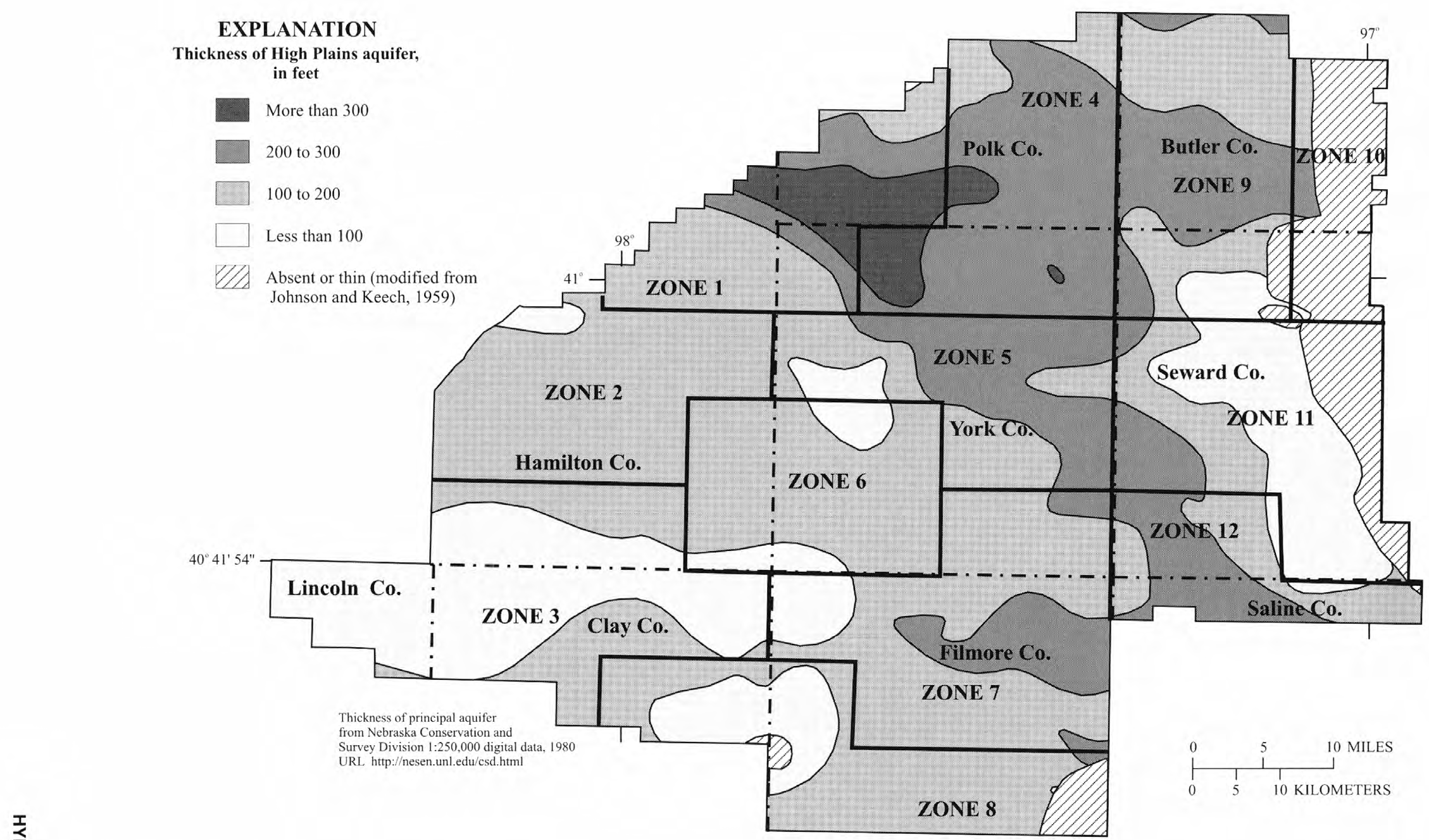

Figure 5. Thickness of High Plains aquifer and management zones, Upper Big Blue Natural Resources District, central Nebraska. 
largest thickness (more than $200 \mathrm{ft}$ ) can be found in parts of Butler, Fillmore, Hamilton, Polk, Saline, Seward, and York Counties. These thick sequences mainly occur in major paleovalleys in the bedrock surface. The High Plains aquifer is less than $100 \mathrm{ft}$ thick in parts of Adams, Clay, Fillmore, Hamilton, Saline, Seward, and York Counties. The High Plains aquifer is thin or absent in parts of Butler, Clay, Fillmore, and Seward Counties. Here the undifferentiated glacial till aquifer (table 2) is present and consists mainly of glacial till with small lenses of sand and gravel, which yield only small amounts of water.

The shallow and deep parts of the High Plains aquifer generally are unconfined in most of Hamilton County, and parts of York, northern Clay, and Adams Counties. In a part of Seward County, only the shallow unconfined part of the High Plains aquifer is present. The deep part of the High Plains aquifer generally is confined in the southern part of York County, most of Fillmore County, the central part of Clay County, the eastern part of Polk County, Butler County, eastern Seward County, and northern Saline County. Water may flow at land surface to a limited extent along the valley of the West Fork of the Big Blue River (fig. 1).

In some parts of the study area, the infiltration of water to the regional water table is retarded by extensive silt and clay lenses of low permeability, creating perched water-table conditions. For example, perched conditions occur near Rising City in Butler County and in northeastern Fillmore County (fig. 1) (Gottula and Link, 1992).

The sources of water to the High Plains aquifer in the study area include ground water by underflow from areas west of the study area, by deep percolation of water from precipitation and irrigation, and by surface-water recharge when the stream stage is higher than the water table where streams are in hydraulic connection with the ground water. Yields from wells screened in the High Plains aquifer locally can exceed $1,000 \mathrm{gal} / \mathrm{min}$.

\section{Bedrock}

The Dakota aquifer is another source of water supply in the study area, although the mineralized character of the water limits its use as a source for potable water supply. The Dakota aquifer (table 2) is used for water supply only in the eastern part of the study area, where the aquifer is relatively shallow and the High Plains aquifer generally is thin or absent (fig. 5). Yields from wells screened in the Dakota aquifer vary from a few gallons to $400 \mathrm{gal} / \mathrm{min}$. These variable yields result from the interbedded character of this hydrogeologic unit (Keech, 1978). The source of ground water is thought to be mainly by under flow.

\section{Water-Table Altitude}

The water-table altitude from the spring of 1996 (fig. 6) generally indicates west to east groundwater flow. Water-table contours are inferred in the extreme eastern part of the area, underlain by glacial till, because there are few wells available for waterlevel measurement. The configuration of the water table generally mirrors the topography. Because of artesian conditions in a part of the area near Beaver Crossing (fig. 1) and perched aquifer conditions locally throughout the study area, the contour lines in figure 6 may reflect these conditions rather than water-table conditions in some areas. The watertable gradient generally ranges from about 4 to $25 \mathrm{ft} / \mathrm{mi}$ and averages about $7 \mathrm{ft} / \mathrm{mi}$. The depth to the water table varies from less than $1 \mathrm{ft}$ in stream valleys to more than $200 \mathrm{ft}$ along the divide between the Platte (north of the study area) and Big Blue Rivers near David City (fig. 1).

Throughout the year, the water-table configuration varies considerably depending upon rates of discharge and recharge at any given time. Rates of recharge depend upon climatic conditions, whereas rates of discharge depend upon climatic conditions and water use. Rates of recharge from precipitation are estimated to be 3 to $5 \mathrm{in} / \mathrm{yr}$ (Dugan and Zelt, in press). Since predevelopment (1950), the water level has risen $22.6 \mathrm{ft}$ in a well in the central part of Polk County for unknown reasons. Since predevelopment (1950), the water level has declined $31 \mathrm{ft}$ in a well in the northwestern part of Fillmore County and at least $5 \mathrm{ft}$ in a large part of Hamilton, Clay, and Fillmore Counties, and in a small part of south-central York and west central Seward Counties (H.H. Chen, U.S. Geological Survey, written commun., 1998). 


\section{EXPLANATION}

-1500- Water-table contour- Shows altitude of water table; Dashed where approximately located; Contour interval 50 feet; Datum is sea level

- $\quad$ Observation well used for control

$\rightarrow$ Ground-water-flow direction

_ - - County boundary

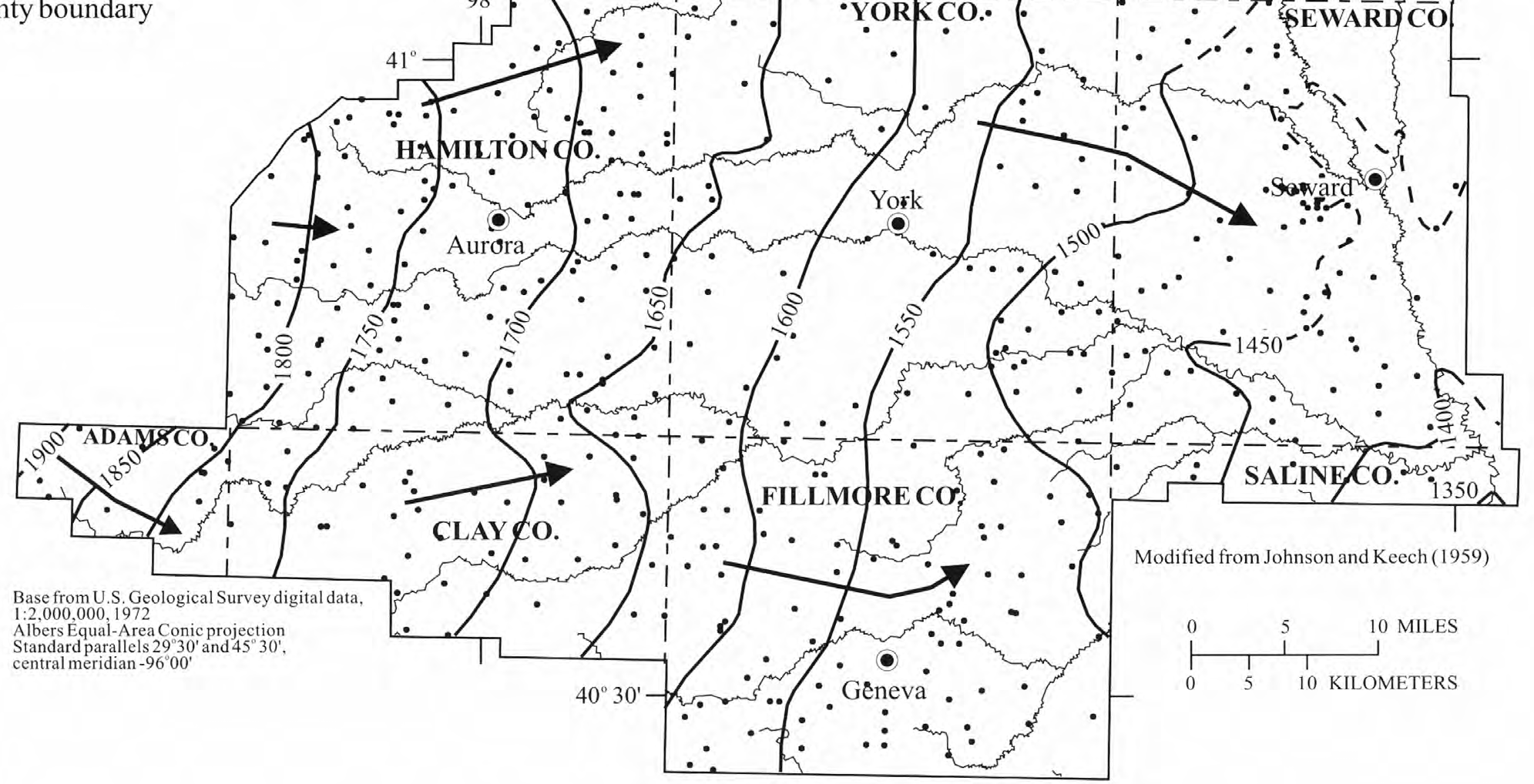

Figure 6. Configuration of water-table altitude, Upper Big Blue Natural Resources District, central Nebraska, spring 1996. 


\section{Hydraulic Properties}

Hydraulic conductivity is a measure of the ability of an aquifer to transmit water and largely is dependent on the nature of the pore space and, thus, the particle-size distribution of the deposits that constitute the aquifer. In most of the study area, the hydraulic conductivity varies from less than 25 to $100 \mathrm{ft} / \mathrm{d}$ (Pettijohn and Chen, 1983b). Estimates of transmissivity are obtained by multiplying the thickness of the aquifer by hydraulic conductivity. The transmissivity of the High Plains aquifer in the study area is estimated to range from about 0 to about 45,000 $\mathrm{ft}^{2} / \mathrm{d}$ (Pettijohn and Chen, 1983a, 1983b). Areas with transmissivities less than 7,000 $\mathrm{ft}^{2} / \mathrm{d}$ usually cannot provide large well yields (more than $750 \mathrm{gal} / \mathrm{min}$ ), and areas with transmissivities larger than $14,000 \mathrm{ft}^{2} / \mathrm{d}$ can sustain large well yields (Keech, 1978).

Specific yield is a measure of the ability of the aquifer to yield water by gravity. The specific yield varies from approximately 5 percent in an area near Seward and south of David City to 25 percent in several small areas scattered across the study area (Pettijohn and Chen, 1983b). The specific yield multiplied by the saturated thickness of the aquifer provides an estimate of the volume of water that the aquifer would yield in an area if the aquifer would be drained by gravity. The volume recoverable from a square mile of aquifer in the study area is estimated to vary from 3,200 acre-ft near Seward to 48,000 acre-ft near Rising City.

The water probably moves at widely differing rates among the shallow and deep parts of the High Plains aquifer and within parts of the High Plains aquifer. The hydraulic conductivity of the shallow part of the High Plains aquifer in the Platte River Valley near Grand Island was determined to be about $134 \mathrm{ft} / \mathrm{d}$ and, assuming porosity is 28 percent and the gradient was $7 \mathrm{ft} / \mathrm{mi}$, the average ground-water velocity was estimated to be about $0.63 \mathrm{ft} / \mathrm{d}$ (Lugn and Wenzel, 1938).

The High Plains aquifer and parts of the Big Blue River and its tributaries (fig. 1) are hydraulically connected (Keech, 1978). The High Plains aquifer and Plum Creek do not appear to be hydraulically connected (fig. 1) in Butler County
(Ginsberg, 1983). Also, there is likely little hydraulic interaction between streams and ground water in Seward County, especially between the High Plains aquifer and the Big Blue River.

\section{MONITORING APPROACH}

During the first year of the study (1995), a thorough evaluation of soils, land use, geology, and hydrology in the study area was conducted in part through analysis of spatial data layers with a geographic information system. Based on this evaluation, a ground-water monitoring network of about 200 wells was proposed. The network included (1) domestic and registered irrigation, municipal, and industrial wells; and (2) eight newly constructed nested-well sites, each consisting of two or three nested wells, screened at various depths assumed to represent the hydrogeologic conditions in the study area (two nested-well sites with three separate monitoring wells and six nested-well sites with two separate nested wells for a total of 18 wells). Samples collected from the unsaturated zone near the eight nested-well sites completed the monitoring approach.

\section{Ground Water}

\section{Domestic and Registered Wells}

The domestic and registered wells included in the proposed ground-water-quality monitoring network were selected using a modified, randomspatial approach, from 96 domestic wells and about 11,000 registered wells in the UBBNRD. A list of domestic wells was derived from a survey of rural residents who submit water samples annually to the UBBNRD for water-quality analysis; domestic wells were included in the selection process if the wellconstruction was known (58 domestic wells). The registered wells included all irrigation, municipal, and industrial wells on file with the Nebraska Department of Water Resources (unpublished data from Nebraska Department of Water Resources, written commun., 1991). The modified randomspatial approach was applied to two groups of wells: (1) domestic and nearby registered wells (within 750 feet), and (2) registered wells that are not near domestic wells (within 750 feet). 
The modified, random-spatial approach for selecting domestic and nearby registered wells consisted of assigning a random number (1) to each township and range in which the domestic wells were located, and then (2) to each of the 58 included domestic wells. The townships and ranges with the 58 smallest random numbers were the selected areas for sampling. Within each of the selected townships and ranges, the domestic well with the smallest random number was chosen for sampling. Nearby registered wells of approximately the same depth also were selected for sampling. If, during the first year of sampling, the nitrate concentration in water from the domestic well would be larger than the concentration in the nearby irrigation well, then the domestic well would be dropped from the network because of possible point-source contamination. The registered well would be retained in the network. If the nitrate concentrations in the domestic and nearby registered well were similar, the domestic well would be retained in the network, and an additional registered well screened in the same geologic unit, but not located near the domestic well, was randomly selected for sampling. Using this methodology, 116 domestic and registered wells were selected to be included in the monitoring network.

The modified random-spatial approach also was used to select an additional 96 registered wells that were not near the domestic wells. This approach consisted of (1) arranging the registered wells into eight groups based on well depth, (2) determining a target number of townships and ranges needed to represent adequately the hydrogeologic unit the wells are completed in based on the number of wells in each group; (3) identifying the townships and ranges where the registered wells were located for each of the eight groups; (4) assigning a number randomly to each identified township and range; and (5) assigning a number randomly to each well. The previously determined target number of townships and ranges needed were selected for each group (ranging from 5 to 20). Within each of the selected townships and ranges, the registered well with the smallest random number was selected.

The resulting set of registered wells were screened in the office and in the field to determine their suitability for sampling. The selection criteria included an acceptable drillers' log; complete wellconstruction information; a continuous well screen that is in only one hydrogeologic unit; an accessible tap that is near the wellhead; and permission from the well owner to sample. If a well did not meet the selection criteria, an alternate registered well in that township and range with a similar depth (within plus or minus $50 \mathrm{ft}$ ) was selected and screened. If a well selected as part of the monitoring program has to be replaced in the future because of well abandonment or other issues, a new well fitting the selection criteria mentioned previously should be selected, of similar depth and nearest the well originally selected.

Following these selection procedures, 206 wells were sampled in 1995 . These 206 wells were reviewed in early 1996 for inclusion in the proposed ground-water monitoring network, based on their location, local geology, well completion, and proximity of potential point sources of contamination. Wells were omitted if (1) wells completed at similar depth (within about $20 \mathrm{ft}$ ) were located within $6 \mathrm{mi}$ of each other; (2) wells were screened in both unconfined and confined parts of the High Plains aquifer; (3) the well construction appeared unsuitable; or (4) the nitrate concentration could be affected by a potential point source nearby. Spatial gaps in data were addressed by selecting additional monitoring sites within these areas.

Based on this review, 163 of the 206 wells sampled in 1995, and 48 new wells, were sampled in 1996. Ten of these wells were resampled in 1997 for quality assurance (QA) and quality control (QC) purposes. A review of all the data was done in 1997. After re-analysis of data for all the wells sampled since 1995, 197 domestic and registered wells were proposed for the ground-water monitoring network in the study area (fig. 7 and table 4). The wells comprising the proposed ground-water monitoring network reflect the ranges of depths of registered wells and are considered representative of the existing well distribution, hydrogeologic conditions, and water-quality conditions in the study area. 


\section{EXPLANATION}

Registered-well location and identification number (table 4) with color illustrating depth, in feet

Shallow part of the High Plains aquifer

${ }^{206}$ Unknown depth

601 Less than or equal to 75

311 More than 75 to 150

320 More than 150 to 225

108 More 225 to 300

107 More than 300

Deep part of the High Plains aquifer

306 More than 75 to 150

318 More than 150 to 225

319 More 225 to 300

708 More than 300

Mixed part of the High Plains aquifer

${ }^{308}$ More than 150 to 225

Greenhorn Formation or

Dakota aquifer

${ }^{1113}$ More than 300

1 Nested well site and number (table 5)

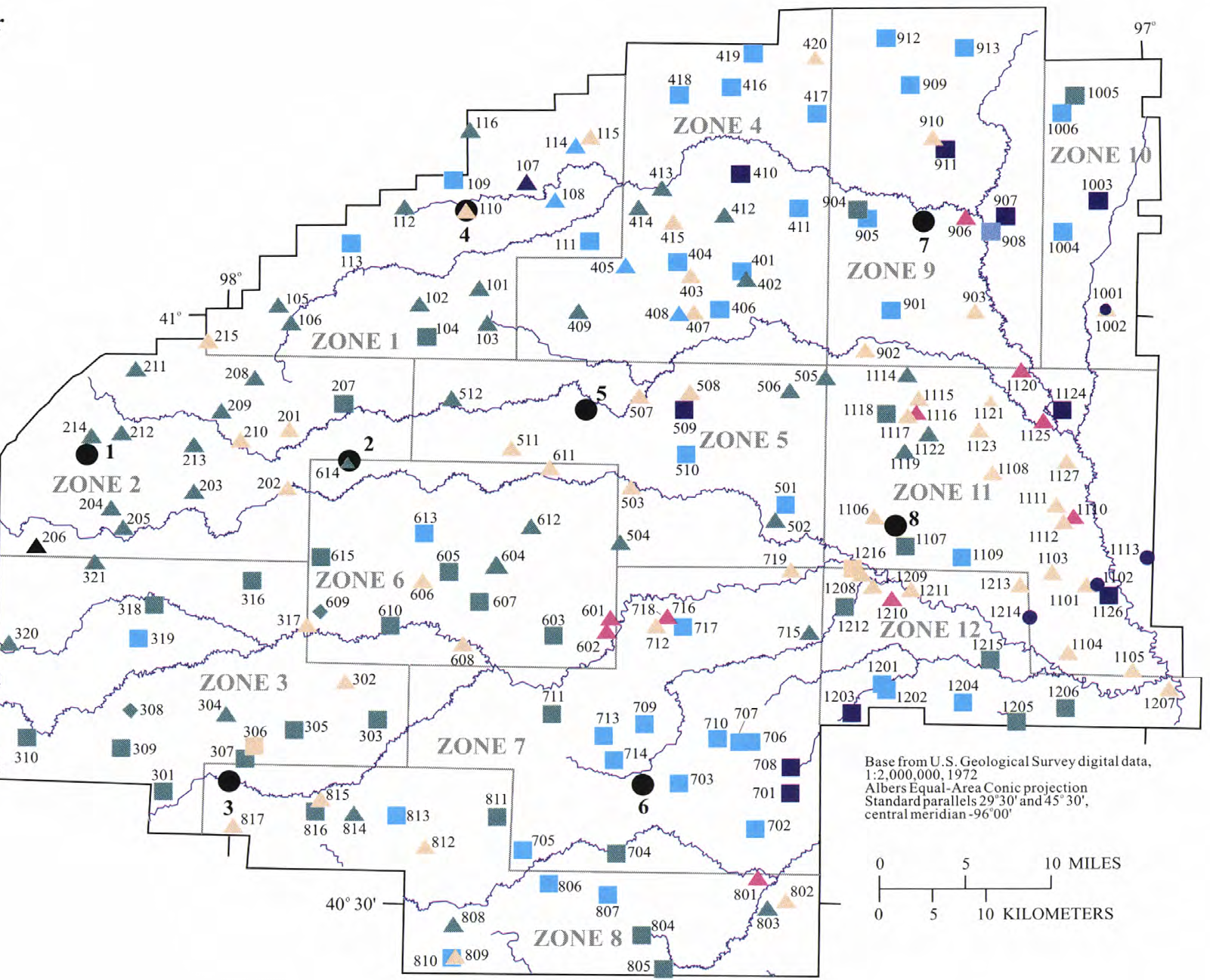

Figure 7. Ground-water-quality monitoring network indicating well depth, aquifer in which wells are completed, and locations of nested wells, Upper Big Blue River Natural Resources District, central Nebraska. 
Table 4. Well information for ground-water-quality monitoring network of domestic and registered wells, Upper Big Blue Natural Resources District, central Nebraska

[ft, feet; bls, below land surface; A, shallow part of High Plains aquifer; B, deep part of High Plains aquifer; M, undifferentiated part of High Plains aquifer;

DA, Dakota aquifer or Greenhorn Formation (well 1001); UF, unconfined part of High Plains aquifer; CF, confined part of High Plains aquifer; NA, not applicable;

I, registered irrigation well; D. domestic well; C, registered industrial well; P, registered public-water-supply well; --, data not available]

\begin{tabular}{|c|c|c|c|c|c|c|c|c|c|c|c|}
\hline $\begin{array}{l}\text { Well } \\
\text { ident- } \\
\text { ifica- } \\
\text { tion } \\
\text { num- } \\
\text { ber } \\
\text { (fig. } \\
7 \text { ) }\end{array}$ & $\begin{array}{l}\text { Water- } \\
\text { bearing } \\
\text { zone }\end{array}$ & $\begin{array}{l}\text { Hydro- } \\
\text { geo- } \\
\text { logic } \\
\text { cond- } \\
\text { ition }\end{array}$ & $\begin{array}{l}\text { Well } \\
\text { type }\end{array}$ & $\begin{array}{l}\text { U.S. Geological } \\
\text { Survey station } \\
\text { identification } \\
\text { number }\end{array}$ & $\begin{array}{l}\text { Well } \\
\text { registration } \\
\text { number }\end{array}$ & $\begin{array}{l}\text { Latitude and } \\
\text { longitude }\end{array}$ & $\begin{array}{l}\text { Location } \\
\text { (fig. 2) }\end{array}$ & $\begin{array}{l}\text { Land surface } \\
\text { altitude } \\
\text { (ft above } \\
\text { sea level) }\end{array}$ & $\begin{array}{l}\text { Well } \\
\text { depth } \\
\text { (bls) }\end{array}$ & $\begin{array}{l}\text { Depth } \\
\text { of } \\
\text { screen } \\
\text { (ft bls) }\end{array}$ & $\begin{array}{l}\text { Bottom } \\
\text { of } \\
\text { screen } \\
\text { (ft bls) }\end{array}$ \\
\hline 0101 & A & UF & I & 410117097451901 & G-00765 & 4101170974519 & T12NR04W10DDBB & 1,732 & 196 & 100 & 196 \\
\hline 0102 & A & UF & I & 410024097491801 & G-66189 & 4100240974918 & T12NR04W18CACC & 1,748 & 209 & 105 & 209 \\
\hline 0103 & A & UF & I & 405930097444401 & G-17488 & 4059300974444 & T12NRW23CCAA & 1,730 & 181 & 129 & 181 \\
\hline 0104 & B & UF & I & 405843097484601 & G-03497 & 4058430974846 & T12NR04W30DBDD & 1,770 & 221 & 157 & 221 \\
\hline 0105 & A & UF & I & 410013097584301 & G-71723 & 4100130975843 & T12NR06W14CCCC & 1,818 & 200 & 120 & 200 \\
\hline 0106 & A & UF & $\mathrm{D}$ & 405915097573401 & -- & 4059150975734 & T12NR06W25BBBC & 1,790 & 155 & -- & -- \\
\hline 0107 & A & UF & I & 410644097421801 & G-61947 & 4106440974218 & T13NR03W 7BBDD & 1,716 & 364 & 107 & 363 \\
\hline 0108 & A & UF & I & 410551097402301 & G-02852 & 4105510974023 & T13NR03W16CBAB & 1,700 & 262 & 202 & 262 \\
\hline 0109 & B & UF & I & 410657097471301 & G-57880 & 4106570974713 & T13NR04W 9BBDD & 1,760 & 277 & 205 & 276 \\
\hline 0110 & A & UF & D & 410513097461801 & -- & 4105130974618 & T13NR04W21AADD & 1,730 & 125 & -- & -- \\
\hline 0111 & B & UF & I & 410346097444001 & G-23684 & 4103400974440 & T13NR04W26CDBC & 1,720 & 260 & 170 & 258 \\
\hline 0112 & A & UF & D & 410521097502601 & -- & 4105210975026 & T13NR05W24BABB & 1,756 & 160 & -- & -- \\
\hline 0113 & B & UF & I & 410327097535801 & G-10894 & 4103270975358 & T13NR05W33BCAA & 1,773 & 230 & 169 & 229 \\
\hline 0114 & A & UF & I & 410840097390401 & G-19788 & 4108400973904 & T14NR03W25BCAA & 1,665 & 240 & 176 & 240 \\
\hline 0115 & A & UF & I & 410906097380401 & G-22451 & 4109060973804 & T14NR03W26CCBB & 1,745 & 129 & 100 & 124 \\
\hline 0116 & A & UF & $\mathrm{D}$ & 410922097460701 & -- & 4109220974607 & T14NR04W27BCCD & 1,748 & 175 & -- & -- \\
\hline 0201 & A & UF & I & 405351097575001 & G-17816 & 4053510975750 & T09NR06W23DACC & 1,750 & 134 & 74 & 134 \\
\hline 0202 & A & UF & I & 405050097575301 & G-04736 & 4050500975753 & T10NR06W11DCAA & 1,745 & 142 & 74 & 142 \\
\hline 0203 & A & UF & D & 405034098040901 & -- & 4050340980404 & T10NR07W13BBAA & 1,821 & 200 & -- & -- \\
\hline 0204 & A & UF & I & 404939098093401 & G-03230 & 4049390980934 & T10NR07W19ABBA & 1,850 & 174 & 91 & 171 \\
\hline 0205 & A & $\mathrm{CF}$ & I & 404840098084301 & G-24000 & 4048400980843 & T10NR07W29BCAA & 1,830 & 218 & 140 & 216 \\
\hline 0206 & A & UF & I & 405036098143201 & A- 05410 & 4050360981432 & T10NR08W16BA & 1,920 & -- & -- & -- \\
\hline 0207 & B & UF & D & 405513097541201 & -- & 4055130975412 & T11NR05W17DADD & 1,751 & 190 & -- & -- \\
\hline 0208 & A & UF & I & 405628098001101 & G-59098 & 4056280980011 & T11NR06W 9AACC & 1,808 & 221 & 130 & 221 \\
\hline 0209 & A & UF & I & 405445098022201 & G-38179 & 4054450980222 & T11NR06W19ADBA & 1,822 & 203 & 150 & 203 \\
\hline 0210 & A & UF & $\mathrm{D}$ & 405315098010401 & -- & 4053150980104 & T11NR06W29DD & 1,801 & 140 & -- & -- \\
\hline 0211 & A & UF & I & 405648098080701 & G-73272 & 4056480980807 & T11NR07W 5DO & 1,849 & 200 & 125 & 200 \\
\hline 0212 & A & UF & I & 405332098085901 & G-09984 & 4053320980859 & T11NR07W30DA & 1,855 & 183 & 123 & 183 \\
\hline 0213 & A & UF & I & 405259098040801 & G-26384 & 4052590980408 & T11NR07W36BBDD & 1,820 & 202 & 138 & 202 \\
\hline 0214 & A & UF & I & 405320098105801 & G-07374 & 4053200981058 & T11NR08W25CO & 1,856 & 195 & 150 & 195 \\
\hline 0215 & A & UF & $\mathrm{D}$ & 405818098032201 &.- & 4058180980322 & T12NR07W36AADD & 1,832 & 145 & - & -- \\
\hline 0301 & B & CF & I & 403511098054001 & G-56699 & 4035110980540 & T07NR07W10DBDD & 1,791 & 201 & 136 & 201 \\
\hline 0302 & A & UF & I & 404059097534801 & G-40014 & 4040590975348 & T08NR05W 9BBAA & 1,741 & 136 & 91 & 136 \\
\hline 0303 & B & UF & I & 403904097513401 & G-02881 & 4039040975134 & T08NR05W23BO & 1,720 & 171 & 108 & 171 \\
\hline 0304 & A & UF & $\mathrm{D}$ & 403913098013601 & -- & 4039130980136 & T08NR06W20BA & 1,781 & 160 & -- & -- \\
\hline 0305 & B & UF & I & 403827097570501 & G-31384 & 4038270975705 & T08NR06W24CD & 1,760 & 215 & 157 & 215 \\
\hline 0306 & B & $\mathrm{CF}$ & $\mathrm{D}$ & 403734097594401 & -- & 4037340975944 & T08NR06W28DD & 1,771 & 140 & 100 & 140 \\
\hline 0307 & B & UF & I & 403656098001801 & G-62766 & 4036560980018 & T08NR06W33CDAA & 1,770 & 201 & 136 & 201 \\
\hline 0308 & M & UF & I & 403917098075801 & G-14577 & 4039170980758 & T08NR07W20ABAA & 1,823 & 190 & 130 & 190 \\
\hline 0309 & B & $\mathrm{CF}$ & I & 403721098083101 & G-14392 & 4037210980831 & T08NR07W32BD & 1,824 & 184 & 108 & 184 \\
\hline 0310 & B & $\mathrm{CF}$ & I & 403747098144601 & G-25125 & 4037470981446 & T08NR08W29DD & 1,872 & 200 & 152 & 200 \\
\hline
\end{tabular}


Table 4. Well information for ground-water-quality monitoring network of domestic and registered wells, Upper Big Blue Natural Resources District, central Nebraska--Continued

\begin{tabular}{|c|c|c|c|c|c|c|c|c|c|c|c|}
\hline $\begin{array}{l}\text { Well } \\
\text { ident- } \\
\text { ifica- } \\
\text { tion } \\
\text { num- } \\
\text { ber } \\
\text { (fig. } \\
7 \text { ) }\end{array}$ & $\begin{array}{l}\text { Water- } \\
\text { bearing } \\
\text { zone }\end{array}$ & $\begin{array}{l}\text { Hydro- } \\
\text { geo- } \\
\text { logic } \\
\text { cond- } \\
\text { ition }\end{array}$ & $\begin{array}{l}\text { Well } \\
\text { type }\end{array}$ & $\begin{array}{l}\text { U.S. Geological } \\
\text { Survey station } \\
\text { identification } \\
\text { number }\end{array}$ & $\begin{array}{l}\text { Well } \\
\text { registration } \\
\text { number }\end{array}$ & $\begin{array}{l}\text { Latitude and } \\
\text { longitude }\end{array}$ & $\begin{array}{l}\text { Location } \\
\text { (fig. 2) }\end{array}$ & $\begin{array}{l}\text { Land surface } \\
\text { altitude } \\
\text { (ft above } \\
\text { sea level) }\end{array}$ & $\begin{array}{l}\text { Well } \\
\text { depth } \\
\text { (bls) }\end{array}$ & $\begin{array}{l}\text { Depth } \\
\text { of } \\
\text { screen } \\
\text { (ft bls) }\end{array}$ & $\begin{array}{l}\text { Bottom } \\
\text { of } \\
\text { screen } \\
\text { (ft bls) }\end{array}$ \\
\hline 0311 & A & UF & I & 404038098184301 & G-20502 & 4040380981843 & T08NR09W11CAAA & 1,894 & 135 & 119 & 135 \\
\hline 0314 & B & $\mathrm{CF}$ & I & 403912098241501 & G-24755 & 4039120982415 & T08NR10W23AACD & 1,954 & 166 & 102 & 166 \\
\hline 0315 & B & $\mathrm{CF}$ & $\mathrm{D}$ & 403814098240401 & -- & 4038140982404 & T08NR10W26ADAA & 1,940 & 180 & -- & -- \\
\hline 0316 & B & $\mathrm{CF}$ & I & 404603098000501 & G-15814 & 4046030980005 & T09NR06W 9AB & 1,785 & 184 & 124 & 184 \\
\hline 0317 & A & UF & I & 404351097562401 & G-25075 & 4043510975624 & T09NR06W24DADD & 1,721 & 122 & 48 & 122 \\
\hline 0321 & A & UF & I & 404653098103301 & G-22284 & 4046530981033 & T10NR08W 1ABAA & 1,877 & 201 & 108 & 201 \\
\hline 0401 & B & $\mathrm{CF}$ & I & 410220097275001 & G-22979 & 4102200972750 & T12NR01W 5CBBB & 1,639 & 290 & 209 & 290 \\
\hline 0402 & A & UF & D & 410156097273001 & -- & 4101560972730 & T12NR01W 5CDCC & 1,625 & 172 & -- & -- \\
\hline 0403 & A & UF & D & 410204097311501 & -- & 4102040973115 & T12NR02W 2CCBC & 1,646 & 150 & -- & -- \\
\hline 0404 & B & UF & I & 410246097320801 & G-18610 & 4102460973208 & T12NR02W 3BABB & 1,660 & 251 & 179 & 251 \\
\hline 0405 & A & UF & I & 410234097353501 & G-04413 & 4102340973535 & T12NR02W 6BDDD & 1,688 & 247 & 130 & 247 \\
\hline 0406 & B & $\mathrm{CF}$ & I & 410024097291601 & G-54205 & 4100220972916 & T12NR02W13DBDD & 1,620 & 245 & 153 & 245 \\
\hline 0407 & A & UF & $\mathrm{D}$ & 410011097305901 & -- & 4100110973059 & T12NR02W14CDCC & 1,639 & 150 & -- & -- \\
\hline 0408 & A & UF & I & 410008097515801 & G-71772 & 4100080973158 & T12NR02W22BABA & 1,645 & 230 & 120 & 230 \\
\hline 0409 & A & UF & I & 410010097384101 & G-13558 & 4100100973841 & T12NR03W22ABBB & 1,695 & 152 & 116 & 152 \\
\hline 0416 & B & UF & I & 411145097284301 & G-21311 & 4111450972843 & T14NR01W 7CCAA & 1,688 & 242 & 194 & 242 \\
\hline 0417 & B & $\mathrm{CF}$ & I & 411027097225801 & G-09174 & 4110270972258 & T14NR01W24BBDC & 1,620 & 233 & 188 & 233 \\
\hline 0418 & B & $\mathrm{CF}$ & I & 411119097321101 & G-11375 & 4111190973211 & T14NR02W15BCBA & 1,668 & 246 & 192 & 246 \\
\hline 0419 & B & $\mathrm{CF}$ & I & 411329097271701 & G-33346 & 4113290972717 & T15NR01W32CADD & 1,650 & 250 & 184 & 249 \\
\hline 0420 & A & UF & D & 411319097230801 & -- & 4113190972308 & T15NR01W36CCDD & 1,638 & 140 & -- & -- \\
\hline 0501 & B & CF & I & 405023097244201 & G-74345 & 4050230972442 & T10NR01W15AACC & 1,599 & 300 & 200 & 300 \\
\hline 0502 & A & UF & I & 404936097252101 & G-21442 & 4049360972521 & T10NR01W22BB & 1,585 & 165 & 85 & 165 \\
\hline 0503 & A & UF & I & 405110097345901 & G-40889 & 4051100973459 & T10NR02W 7ADCC & 1,589 & 100 & 35 & 100 \\
\hline 0504 & A & UF & I & 404819097354301 & G-05619 & 4048220973538 & T10NR02W30CB & 1,645 & 156 & 110 & 156 \\
\hline 0505 & A & UF & I & 405409097282401 & G-62918 & 4056580972208 & T11NR01W 1DACD & 1,604 & 154 & 101 & 153 \\
\hline 0506 & A & UF & I & 405615097242901 & G-63219 & 4056150972429 & T11NR01W10DABA & 1,620 & 155 & 95 & 155 \\
\hline 0507 & A & UF & $\mathrm{D}$ & 405552097343101 & -. & 4055520973431 & T11NR02W 8CDCC & 1,610 & 80 & -- & -- \\
\hline 0508 & A & UF & $\mathrm{D}$ & 405606097311001 & -- & 4056060973110 & T11NR02W11CBCC & 1,641 & 130 & -- & -- \\
\hline 0509 & B & $\mathrm{CF}$ & I & 405511097313201 & G-40396 & 4055110973132 & T11NR02W11CBCC & 1,641 & 313 & 120 & 313 \\
\hline 0510 & B & $\mathrm{CF}$ & I & 405255097312201 & G-18998 & 4052550973122 & T11NR02W35BBDD & 1,622 & 136 & 76 & 136 \\
\hline 0511 & A & UF & I & 405305097430001 & G-06930 & 4053050974300 & T11NR04W36ACAA & 1,703 & 110 & 62 & 110 \\
\hline 0512 & A & UF & I & 405537097470201 & G-14953 & 4055370974702 & T11NR04W16BDDD & 1,714 & 181 & 105 & 181 \\
\hline 0601 & A & UF & I & 404428097361401 & G-16189 & 4044280973614 & T9NR30W24 AABC & 1,578 & 63 & 32 & 62 \\
\hline 0602 & A & UF & I & 404347097362701 & G-11012 & 4043470973627 & T09NR03W24DDBC & 1,573 & 67 & 27 & 67 \\
\hline
\end{tabular}


Table 4. Well information for ground-water-quality monitoring network of domestic and registered wells, Upper Big Blue Natural Resources District, central Nebraska--Continued

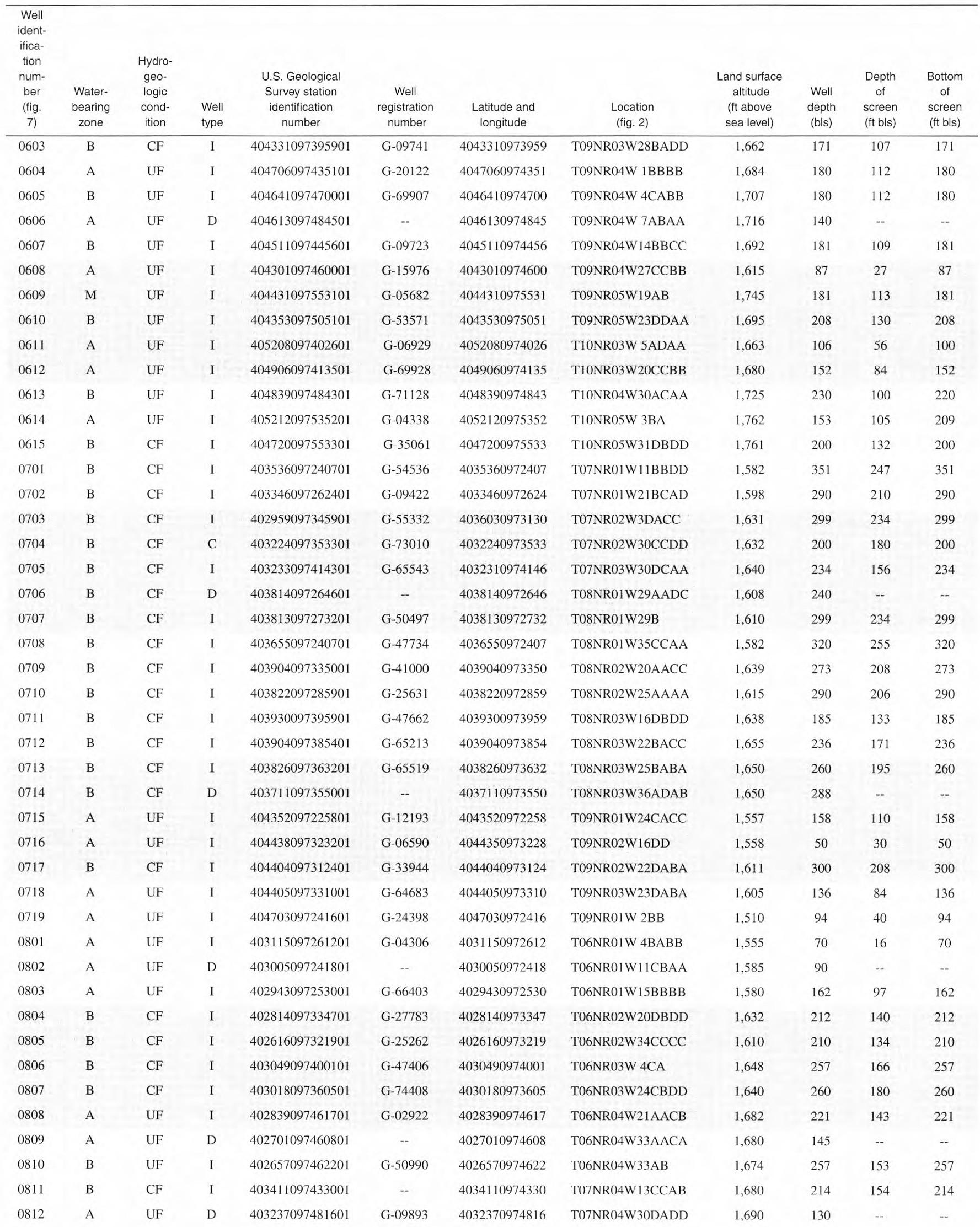


Table 4. Well information for ground-water-quality monitoring network of domestic and registered wells, Upper Big Blue Natural Resources District, central Nebraska--Continued

\begin{tabular}{|c|c|c|c|c|c|c|c|c|c|c|c|}
\hline $\begin{array}{c}\text { Well } \\
\text { ident- } \\
\text { ifica- } \\
\text { tion } \\
\text { num- } \\
\text { ber } \\
\text { (fig. } \\
7 \text { ) }\end{array}$ & $\begin{array}{l}\text { Water- } \\
\text { bearing } \\
\text { zone }\end{array}$ & $\begin{array}{l}\text { Hydro- } \\
\text { geo- } \\
\text { logic } \\
\text { cond- } \\
\text { ition }\end{array}$ & $\begin{array}{l}\text { Well } \\
\text { type }\end{array}$ & $\begin{array}{l}\text { U.S. Geological } \\
\text { Survey station } \\
\text { identification } \\
\text { number }\end{array}$ & $\begin{array}{l}\text { Well } \\
\text { registration } \\
\text { number }\end{array}$ & $\begin{array}{l}\text { Latitude and } \\
\text { longitude }\end{array}$ & $\begin{array}{l}\text { Location } \\
\text { (fig. 2) }\end{array}$ & $\begin{array}{c}\text { Land surface } \\
\text { altitude } \\
\text { (ft above } \\
\text { sea level) }\end{array}$ & $\begin{array}{l}\text { Well } \\
\text { depth } \\
\text { (bls) }\end{array}$ & $\begin{array}{l}\text { Depth } \\
\text { of } \\
\text { screen } \\
\text { (ft bls) }\end{array}$ & $\begin{array}{l}\text { Bottom } \\
\text { of } \\
\text { screen } \\
\text { (ft bls) }\end{array}$ \\
\hline 0813 & $\mathrm{~B}$ & $\mathrm{CF}$ & I & 403413097501301 & G-67711 & 4034100975013 & T07NR05W13DCAA & 1,740 & 260 & 182 & 260 \\
\hline 0814 & A & UF & $\mathrm{D}$ & 403415097530101 & -- & 4034150975301 & T07NR05W16DC & 1,755 & 205 & 192 & 205 \\
\hline 0815 & A & UF & $\mathrm{D}$ & 403456097551601 & G-07849 & 4034560975516 & T07NR05W18AAA & 1,745 & 90 & 75 & 90 \\
\hline 0816 & B & $\mathrm{CF}$ & I & 403418097553501 & G-16027 & 4034180975535 & T07NR05W18DCAD & 1,755 & 205 & 160 & 205 \\
\hline 0817 & A & UF & I & 403330098010101 & G-24009 & 4033300980101 & T07NR06W20DAA & 1,774 & 150 & 95 & 150 \\
\hline 0901 & $\mathrm{~B}$ & $\mathrm{CF}$ & I & 410026097175101 & G-43139 & 4100260971751 & T12NR01E15DCAA & 1,586 & 292 & 193 & 292 \\
\hline 0902 & A & UF & I & 405822097193201 & G-07160 & 4058220971932 & T12NR01E33BBAC & 1,520 & 109 & 37 & 109 \\
\hline 0903 & A & UF & I & 410025097121301 & G-24711 & 4100250971213 & T12NR02E16DCAC & 1,550 & 127 & 87 & 126.5 \\
\hline 0904 & B & $\mathrm{CF}$ & I & 410533097193701 & G-16177 & 4105330972007 & T13NR01E16CCAC & 1,565 & 179 & 119 & 179 \\
\hline 0905 & B & $\mathrm{CF}$ & I & 410507097193001 & G-26880 & 4105070971930 & T13NR01E21BO & 1,604 & 264 & 188 & 264 \\
\hline 0906 & A & UF & $\mathrm{D}$ & 410514097125601 & - & 4105140971256 & T13NR02E20AADD & 1,502 & 40 & -- & -- \\
\hline 0907 & B & $\mathrm{CF}$ & I & 410518097101701 & G-45443 & 4105180971017 & T13NR02E23BBDA & 1,585 & 304 & 199 & 304 \\
\hline 0908 & B & $\mathrm{CF}$ & I & 410431097111501 & G-72305 & 4104310971115 & T13NR02E27BABA & 1,519 & 244 & 143 & 244 \\
\hline 0909 & B & $\mathrm{CF}$ & I & 411159097164501 & G-27404 & 4111590971645 & T14NR01E11ACCC & 1,585 & 227 & 139 & 227 \\
\hline 0910 & A & UF & D & 410918097151201 & -- & 4109180971512 & T14NR02E30CBBC & 1,598 & 110 & - & -. \\
\hline 0911 & B & $\mathrm{CF}$ & I & 410842097142101 & G-57715 & 4108420971421 & T14NR02E31ADBB & 1,595 & 303 & 225 & 303 \\
\hline 0912 & $\mathrm{~B}$ & $\mathrm{CF}$ & I & 411422097182501 & G-04235 & 4114220971825 & T15NR01E27CCBD & 1,620 & 243 & 171 & 243 \\
\hline 0913 & $\mathrm{~B}$ & $\mathrm{CF}$ & I & 411355097131001 & G-73824 & 4113550971310 & T15NR02E32AACC & 1,627 & 243 & 194 & 242 \\
\hline 1001 & DA & NRA & $\mathrm{P}$ & 410034097033401 & G-72288 & 4100340970334 & T12NR03E14CBAC & 1,562 & 368 & 328 & 368 \\
\hline 1002 & A & UF & $\mathrm{P}$ & 410035097033001 & G-28285 & 4100350970330 & T12NR03E14CBAD & 1,563 & 95 & 75 & 95 \\
\hline 1003 & B & $\mathrm{CF}$ & I & 410608097040701 & G-45124 & 4106080970407 & 13NR03E15ABCA & 1,610 & 430 & 300 & 430 \\
\hline 1004 & B & $\mathrm{CF}$ & $\mathrm{D}$ & 410433097062601 & - & 4104330970626 & T13NR03E20DCCD & 1,615 & 285 & -- & - \\
\hline 1005 & B & $\mathrm{CF}$ & I & 411131097054201 & G-73044 & 4111310970542 & T14NR03E 9CDCD & 1,591 & 197 & 177 & 197 \\
\hline 1006 & B & $\mathrm{CF}$ & I & 411038097063501 & G-47019 & 4110380970635 & T14NR03E20ABBB & 1,563 & 240 & 186 & 226 \\
\hline 1101 & A & UF & I & 404627097043601 & G-33780 & 4046270970436 & T09NR03E3CDBB & 1,493 & 112 & 80 & 112 \\
\hline 1102 & DA & NRA & I & 404628097035601 & G-70815 & 4046280970356 & T09NR03E 3DDDB & 1,470 & 358 & 315 & 355 \\
\hline 1103 & A & UF & I & 404704097065501 & G-23634 & 4047040970655 & T09NR03E 5BABA & 1,530 & 140 & 100 & 140 \\
\hline 1104 & A & UF & I & 404259097054801 & G-29058 & 4042590970548 & T09NR03E28CACC & 1,473 & 98 & 66 & 98 \\
\hline 1105 & A & UF & I & 404206097013101 & G-51481 & 4042060970131 & T09NR03E36DDAA & 1,445 & 97 & 32 & 97 \\
\hline 1106 & A & UF & I & 404949097184801 & G-17148 & 4049490971848 & T10NR01E16DDDB & 1,565 & 136 & 88 & 136 \\
\hline 1107 & B & $\mathrm{CF}$ & I & 404819097164001 & G-40262 & 4048190971640 & T10NR01E26DABC & 1,552 & 215 & 150 & 215 \\
\hline 1108 & A & UF & I & 405208097105701 & G-62771 & 4052080971057 & T10NR02E $3 \mathrm{AO}$ & 1,525 & 121 & 77 & 121 \\
\hline 1109 & B & $\mathrm{CF}$ & I & 404748097125701 & G-44369 & 4047480971257 & T10NR02E33BBCC & 1,540 & 290 & 186 & 290 \\
\hline 1110 & A & UF & I & 404957097053201 & G-64121 & 4049570970532 & T10NR03E16DCBB & 1,440 & 50 & 30 & 50 \\
\hline 1111 & A & UF & I & 405031097064101 & G-33025 & 4050310970641 & 10NR03E17ABCC & 1,490 & 100 & 44 & 100 \\
\hline 1112 & A & UF & $\mathrm{D}$ & 404940097061201 & -- & 4049400970612 & 10NR03E20AAAC & 1,483 & 86 & 68 & 86 \\
\hline 1113 & DA & NA & I & 404752097003901 & G-64099 & 4047520970039 & T10NR04E31AACB & 1,471 & 420 & 366 & 420 \\
\hline 1114 & A & UF & I & 405709097164201 & G-52744 & 4057090971642 & T11NR01E 2ACDD & 1,580 & 168 & 104 & 168 \\
\hline 1115 & A & UF & $\mathrm{D}$ & 405556097155601 & -- & 4055560971556 & T11NR01E12CDCC & 1,573 & 90 & - & -- \\
\hline 1116 & A & UF & I & 405512097160501 & G-07142 & 4055120971605 & T11NR01E13CCAA & 1,546 & 61 & -- & -- \\
\hline 1117 & A & UF & I & 405502097163901 & G-46950 & 4055020971639 & T11NR01E14DDCB & 1,593 & 130 & 70 & 130 \\
\hline 1118 & B & $\mathrm{CF}$ & I & 405507097182301 & G-57892 & 4055070971803 & T11NR01E15CCAD & 1,584 & 225 & 160 & 225 \\
\hline 1119 & A & UF & I & 405313097164901 & G-68540 & 4053130971649 & T11NR01E35ABAB & 1,561 & 166 & 101 & 166 \\
\hline 1120 & A & UF & I & 405726097090801 & G-26635 & 4057260970908 & T11NR02E 1BACD & 1,460 & 42 & 22 & 42 \\
\hline 1121 & A & UF & I & 405551097110801 & G-51687 & 4055510971108 & T11NR02E10DCCC & 1,500 & 95 & 51 & 95 \\
\hline
\end{tabular}


Table 4. Well information for ground-water-quality monitoring network of domestic and registered wells, Upper Big Blue Natural Resources District, central Nebraska--Continued

\begin{tabular}{|c|c|c|c|c|c|c|c|c|c|c|c|}
\hline $\begin{array}{c}\text { Well } \\
\text { ident- } \\
\text { ifica- } \\
\text { tion } \\
\text { num- } \\
\text { ber } \\
\text { (fig. } \\
7 \text { ) }\end{array}$ & $\begin{array}{l}\text { Water- } \\
\text { bearing } \\
\text { zone }\end{array}$ & $\begin{array}{c}\text { Hydro- } \\
\text { geo- } \\
\text { logic } \\
\text { cond- } \\
\text { ition }\end{array}$ & $\begin{array}{l}\text { Well } \\
\text { type }\end{array}$ & $\begin{array}{l}\text { U.S. Geological } \\
\text { Survey station } \\
\text { identification } \\
\text { number }\end{array}$ & $\begin{array}{l}\text { Well } \\
\text { registration } \\
\text { number }\end{array}$ & $\begin{array}{l}\text { Latitude and } \\
\text { longitude }\end{array}$ & $\begin{array}{l}\text { Location } \\
\text { (fig. 2) }\end{array}$ & $\begin{array}{c}\text { Land surface } \\
\text { altitude } \\
\text { (ft above } \\
\text { sea level) }\end{array}$ & $\begin{array}{l}\text { Well } \\
\text { depth } \\
\text { (bls) }\end{array}$ & $\begin{array}{l}\text { Depth } \\
\text { of } \\
\text { screen } \\
\text { (ft bls) }\end{array}$ & $\begin{array}{l}\text { Bottom } \\
\text { of } \\
\text { screen } \\
\text { (ft bls) }\end{array}$ \\
\hline 1122 & A & UF & I & 405408097151301 & G-35928 & 4054080971513 & 11NR02E19CCCC & 1,565 & 165 & 105 & 165 \\
\hline 1123 & A & UF & I & 405420097115401 & G-17147 & 4054200971154 & 11NR02E21DDBA & 1,545 & 128 & 92 & 128 \\
\hline 1124 & $\mathrm{~B}$ & $\mathrm{CF}$ & I & 405524097062101 & G-70405 & 4055240970621 & T11NR03E17DCDC & 1,446 & 462 & 402 & 462 \\
\hline 1125 & A & UF & $\mathrm{D}$ & 405457097075501 & -- & 4054510970737 & T11NR03E19BAAB & 1,501 & 70 & -- & $\ldots$ \\
\hline 1126 & $\mathrm{~B}$ & $\mathrm{CF}$ & $\mathrm{D}$ & 404554097031001 & G-85864 & 4045540970310 & T11NR03E23ABBB & 1,522 & 378 & 368 & 378 \\
\hline 1127 & A & UF & $\mathrm{D}$ & 405246097060101 & - & 4052460970601 & T11NR03E33CBBB & 1,481 & 120 & - & - \\
\hline 1201 & B & $\mathrm{CF}$ & I & 404116097180801 & G-65052 & 4041160971808 & T08NR01E 3DBBB & 1,550 & 266 & 201 & 266 \\
\hline 1202 & B & $\mathrm{CF}$ & I & 404058097175001 & G-52169 & 4040580971750 & T08NR01E10AABC & 1,551 & 247 & 182 & 247 \\
\hline 1203 & B & $\mathrm{CF}$ & I & 403945097200701 & G-66719 & 4039450972007 & T08NR01E17AC & 1,530 & 312 & 195 & 312 \\
\hline 1204 & B & $\mathrm{CF}$ & I & 404023097124501 & A-04499R & 4040230971245 & T08NR02E 9CC & 1,532 & 292 & 227 & 292 \\
\hline 1205 & $\mathrm{~B}$ & UF & I & 404930097091401 & G-07141 & 4039240970909 & T08NR02E13CO & 1503 & 220 & 140 & 220 \\
\hline 1206 & B & $\mathrm{CF}$ & I & 404008097055501 & G-58947 & 4040080970555 & T08NR03E16BABB & 1,478 & 195 & 130 & 195 \\
\hline 1207 & A & UF & I & 404109096590601 & G-23552 & 4041090965906 & T08NR04E 4CC & 1,383 & 100 & 40 & 100 \\
\hline 1208 & A & $\mathrm{UF}$ & I & 404653097193501 & G-48066 & 4046530971935 & T09NR01E 4BCAD & 1,474 & 90 & 24 & 89 \\
\hline 1209 & A & UF & $\mathrm{D}$ & 404616097185001 & - & 4046160971850 & T09NR01E 5DDDD & 1,505 & 120 & 65 & 120 \\
\hline 1210 & A & UF & $\mathrm{D}$ & 404538097173401 & - & 4045380971734 & T09NR01E10DDDD & 1,531 & 65 & 20 & 65 \\
\hline 1211 & A & UF & I & 404606097161801 & G-64347 & 4046060971618 & T09NR01E12BCBA & 1,450 & 130 & 104 & 130 \\
\hline 1212 & B & $\mathrm{CF}$ & I & 404511097204001 & G-53766 & 4045110972040 & T09NR01E17BO & 1,525 & 208 & 143 & 208 \\
\hline 1213 & A & UF & I & 404630097090501 & $\mathrm{G}-42251$ & 4046300970905 & T09NR02E 1CDBA & 1,535 & 147 & 94 & 147 \\
\hline 1214 & $\mathrm{DA}$ & NA & I & 404445097082401 & G-38989 & 4044450970824 & T09NR02E13DAAA & 1,546 & 485 & 434 & 485 \\
\hline 1215 & B & $\mathrm{CF}$ & I & 404234097105701 & G-65685 & 4042340971057 & T09NR02E34AO & 1,500 & 154 & 128 & 154 \\
\hline 1216 & B & $\mathrm{CF}$ & I & 404708097201101 & G-73781 & 4047080972011 & T10NR01E32DCDC & 1,465 & 140 & 140 & 100 \\
\hline
\end{tabular}


The number of wells in a management zone, delineated by the UBBNRD, are: 16 in zone 1,15 in zone 2, 21 in zone 3, 20 in zone 4, 12 in zone 5, 15 in zone 6,19 in zone 7,17 in zone 8,13 in zone 9,6 in zone 10, 27 in zone 11, and 16 in zone 12 (fig. 7 and table 4). Of the 197 wells, 104 wells are completed in the shallow part (designated $\mathrm{A}$ in table 4) of the High Plains aquifer, 86 are completed in the deep part of the High Plains aquifer (B), 3 are completed in both parts of this aquifer (M), 1 is completed in the Greenhorn Formation (well 1001) (DA), and 3 are completed in the Dakota aquifer (DA) (fig. 7 and table 4). Of the 193 wells completed in the High Plains aquifer, 123 are screened in an unconfined part of the High Plains aquifer and 70 are screened in a confined part of the High Plains aquifer (table 4). The wells exceeding a depth of $300 \mathrm{ft}$ generally are in the eastern part of the study area (fig. 1, fig. 7). Wells less than $150 \mathrm{ft}$ deep commonly are in the central and eastern parts of the study area (fig. 1, fig. 7). Wells completed in the shallow part of the High Plains aquifer commonly are in Hamilton, Polk, Seward, and York Counties. Wells completed in the deep part of the High Plains aquifer generally are in Adams, Butler, Clay, Fillmore, Polk, York, and Saline Counties. This deep part of the High Plains aquifer generally is unconfined in parts of Clay, Polk, and York Counties. The wells completed in confined parts of the High Plains aquifer generally are in Butler, Fillmore, and Saline Counties (fig. 8 shown later in report).

\section{Nested Wells}

Eight nested-well sites representative of the hydrogeologic conditions in the study area were selected for the installation of 18 nested wells in 1995 (table 5) (fig. 7). Nested-well sites and completion depths were selected based on: the management zones established by the UBBNRD (eight sites for the 12 zones) (Upper Big Blue Natural Resources District, 1994); the presence of irrigated cropland at the site with known amounts of chemical fertilizer and pesticide use; the availability of detailed geologic descriptions of test holes preferrably within $1 \mathrm{mi}$ of the selected sites; the suitability of the location for well construction; and the permission of the landowner(s). Well depths of the nested wells vary from 55 to $250 \mathrm{ft}$, screen lengths in each well vary from 5 to $10 \mathrm{ft}$, and screen slot sizes vary from 0.100 to $0.025 \mathrm{in}$. and are dependent upon the hydrogeologic conditions at the site.

\section{Unsaturated Zone}

Near each nested well, unsaturated-zone samples of unconsolidated sediments were collected from cores that varied in depth from 5 to $90 \mathrm{ft}$ and were analyzed for nitrate (table 6). Near each nestedwell site, one unsaturated-zone-core subsite was selected on irrigated cropland. In addition, samples were collected from duplicate cores at nested-well sites 2 and 3. Additional unsaturated-zone core subsites were selected, based on landscape position or land use, near nested-well sites 3, 4, 6, and 7, to evaluate whether nitrate concentrations in the unsaturated zone vary with landscape position (upland or bottomland) and land use (table 6). Samples varied in depth from 0.25 to 90 feet.

\section{METHODS}

This section presents an overview of the sample-collection procedures of ground-water and sediment samples, laboratory procedures, dataanalysis procedures, and the quality-assurance and quality-control measures implemented during the study.

\section{Sample-Collection Procedures}

\section{Ground-Water Samples}

In 1995, water samples were collected from 206 domestic and registered irrigation, municipal, and industrial wells. In 1996, water samples were collected from 211 domestic and registered irrigation, municipal, and industrial wells. In 1997, 10 domestic and registered wells were resampled. Samples from 18 nested wells at 8 nested-well sites were collected monthly or bimonthly from November 1995 through May 1997. 
Table 5. Locations and descriptions of nested wells, Upper Big Blue Natural Resources District, central Nebraska

[ft, foot; bls, below land surface; A, shallow part of the High Plains aquifer; B, deep part of the High Plains aquifer; UF, unconfined part of the High Plains aquifer; $\mathrm{CF}$, confined part of the High Plains aquifer]

\begin{tabular}{|c|c|c|c|c|c|c|c|c|c|}
\hline $\begin{array}{c}\text { Well- } \\
\text { nest } \\
\text { site } \\
\text { number } \\
\text { (fig. 7) }\end{array}$ & $\begin{array}{l}\text { Nested- } \\
\text { well } \\
\text { identifi- } \\
\text { cation } \\
\text { number }\end{array}$ & $\begin{array}{l}\text { Unique well- } \\
\text { identification } \\
\text { number } \\
\text { representing } \\
\text { latitude, } \\
\text { longitude, and } \\
\text { two-digit } \\
\text { sequence number }\end{array}$ & $\begin{array}{l}\text { Well } \\
\text { registra- } \\
\text { tion } \\
\text { number }\end{array}$ & $\begin{array}{l}\text { Location } \\
\text { (fig. 2) }\end{array}$ & $\begin{array}{l}\text { Esti- } \\
\text { mated } \\
\text { altitude } \\
\text { (ft bls) }\end{array}$ & $\begin{array}{l}\text { Well } \\
\text { depth } \\
(\mathrm{ft})\end{array}$ & $\begin{array}{c}\text { Depth of } \\
\text { screened } \\
\text { interval } \\
\text { (ft bls) }\end{array}$ & $\begin{array}{l}\text { High } \\
\text { Plains } \\
\text { aquifer } \\
\text { part }\end{array}$ & $\begin{array}{c}\text { Hydro- } \\
\text { geologic } \\
\text { condition } \\
\text { of water- } \\
\text { bearing } \\
\text { strata } \\
\text { sampled }\end{array}$ \\
\hline 1 & $1 \mathrm{~A}$ & 405220098111201 & G-086355 & T10N R08W 01 BBBA & 1,860 & 95 & $90-95$ & A & UF \\
\hline 1 & $1 \mathrm{~B}$ & 405220098111202 & G-086356 & T10N R08W 01 BBBA & 1,860 & 205 & $200-205$ & B & $\mathrm{CF}$ \\
\hline 2 & $2 \mathrm{~A}$ & 405220097535201 & G-086357 & T10N R05W 04 BBAA & 1,767 & 107 & $102-107$ & A & UF \\
\hline 2 & $2 \mathrm{~B}$ & 405220097535202 & G-086358 & T10N R05W 04B BAA & 1,767 & 195 & $190-195$ & B & UF \\
\hline 3 & $3 \mathrm{~A}$ & 403549098012501 & G-086359 & T07N R06W 08 ABBB & 1,750 & 100 & $95-100$ & A & UF \\
\hline 3 & 3B & 403549098012502 & G-086360 & T07N R06W 08 ABBB & 1,750 & 165 & $160-165$ & B & UF \\
\hline 4 & $4 \mathrm{~A}$ & 410515097461401 & G-086361 & T13N R04W 21 AADA & 1,720 & 95 & $90-95$ & A & UF \\
\hline 4 & $4 \mathrm{~B}$ & 410515097461402 & G-086362 & T13N R04W 21 AADA & 1,720 & 125 & $120-125$ & B & UF \\
\hline 5 & $5 \mathrm{~A}$ & 405509097380901 & G-086363 & T11N R03W 15 DDDA & 1,650 & 92 & $82-92$ & A & UF \\
\hline 5 & $5 \mathrm{~B}$ & 405509097380903 & G-086364 & T11N R03W 15 DDDA & 1,650 & 233 & $223-233$ & B & $\mathrm{CF}$ \\
\hline 6 & $6 \mathrm{~A}$ & 403603097340301 & G-086365 & T07N R02W 05 DBCD & 1,669 & 150 & $140-150$ & B & $\mathrm{CF}$ \\
\hline 6 & $6 \mathrm{~B}$ & 403603097340302 & G-086366 & T07N R02W 05 DBCD & 1,669 & 247 & $240-247$ & B & $\mathrm{CF}$ \\
\hline 7 & $7 \mathrm{~A}$ & 410459097155601 & G-086367 & T13N R01E 24 CAAB & 1,547 & 55 & $45-55$ & A & UF \\
\hline 7 & $7 \mathrm{~B}$ & 410459097155602 & G-086368 & T13N R01E 24 CAAB & 1,547 & 112 & $102-112$ & B & $\mathrm{CF}$ \\
\hline 7 & $7 \mathrm{C}$ & 410459097155603 & G-086369 & T13N R01E 24 CAAB & 1,547 & 250 & $243-250$ & B & $\mathrm{CF}$ \\
\hline 8 & $8 \mathrm{~A}$ & 404928097173201 & G-086370 & T10N R01E 22 ADAD & 1,560 & 100 & $90-100$ & A & UF \\
\hline 8 & $8 \mathrm{~B}$ & 404928097173202 & G-086371 & T10N R01E 22 ADAD & 1,560 & 130 & $120-130$ & A & UF \\
\hline 8 & $8 \mathrm{C}$ & 404928097173203 & G-086372 & T10N R01E 22 ADAD & 1,560 & 230 & $225-230$ & B & $\mathrm{CF}$ \\
\hline
\end{tabular}

Ground-water samples were collected from domestic, registered, and monitoring wells by USGS and UBBNRD personnel. On site, the well integrity was evaluated, and potential point sources of contamination, such as septic tanks, feedlots, municipal dumps, and industry near the sampled wells, and prevalent land use within a 1-mi radius of the well, were noted on the field sheet. The landsurface altitude was estimated from USGS 7.5minute topographic maps (scale 1:24,000) with accuracy of about $5 \mathrm{ft}$. The location of each well was determined with a global positioning system. Generally, the well locations were determined with an accuracy of less than $25 \mathrm{ft}$ to as much as $50 \mathrm{ft}$.

Water was collected from each well as close to the well head as possible. If a spigot was not present, spigots were installed when possible. Field measurements for specific conductance, $\mathrm{pH}$, water temperature, and dissolved oxygen (when a sample was not collected from a gated pipe) were made at 5 minute intervals with a flow-through chamber.

Water samples were collected after the field measurements stabilized, typically after 15 to 25 minutes. The field measurements were considered stable on the basis of three tolerances: specific conductance remained within 5 percent; $\mathrm{pH}$ remained within 0.1 standard unit; and water temperature remained within $0.2{ }^{\circ} \mathrm{C}$ (degrees Celsius). These procedures were assumed to be sufficient for collecting water samples representative of water-bearing units surrounding the well screen.

At each sample site, water samples collected to be analyzed for dissolved nitrate and nitrite as nitrogen-referred to as such in the tables, but referred to as dissolved nitrate in the remainder of the text and figures - and major ions were filtered immediately through a 0.45 -micrometer membrane 
Table 6. Locations and descriptions of unsaturated-zone sampling subsites, Upper Big Blue Natural Resources District, central Nebraska

[DH denotes unsaturated-zone location, the subsite number is the nested-well site number near which the site is located, and $\mathrm{X}, \mathrm{Y}$, or $\mathrm{Z}$ differentiates between subsites]

\begin{tabular}{|c|c|c|c|c|c|c|}
\hline \multirow{2}{*}{$\begin{array}{l}\text { Local } \\
\text { subsite } \\
\text { identifi- } \\
\text { cation } \\
\text { number }\end{array}$} & \multirow{2}{*}{$\begin{array}{l}\text { Unique sample } \\
\text { location number } \\
\text { representing } \\
\text { latitude and } \\
\text { longitude }\end{array}$} & \multirow{2}{*}{$\begin{array}{l}\text { Location } \\
\text { (fig. 2) }\end{array}$} & \multirow{2}{*}{$\begin{array}{l}\text { Nested- } \\
\text { well site } \\
\text { number } \\
\text { (fig. } 7 \text { ) }\end{array}$} & \multirow{2}{*}{$\begin{array}{l}\text { Physiographic and land-use } \\
\text { characteristics }\end{array}$} & \multicolumn{2}{|c|}{$\begin{array}{c}\text { Nitrogen } \\
\text { applied } \\
\text { (pounds per } \\
\text { acre) }\end{array}$} \\
\hline & & & & & 1995 & 1996 \\
\hline DH1 & 4052180981108 & T10N R08W 01 & 1 & $\begin{array}{l}\text { Sprinkler-irrigated cropland, bottom- } \\
\text { land, minimum tillage, continuous } \\
\text { corn }\end{array}$ & 165 & 165 \\
\hline $\mathrm{DH} 2 \mathrm{X}$ & 4052200975353 & T10N R05W 04 & 2 & $\begin{array}{l}\text { Gravity-irrigated cropland, upland, ridge } \\
\text { till, corn ( } 2 \text { years) and bean (1 year) } \\
\text { rotation }\end{array}$ & 160 & 160 \\
\hline $\mathrm{DH} 2 \mathrm{Y}$ & 4052190975353 & T10N R05W 04 & 2 & $\begin{array}{l}\text { Gravity-irrigated cropland, upland, ridge } \\
\text { till, corn ( } 2 \text { years) and bean ( } 1 \text { year) } \\
\text { rotation }\end{array}$ & 160 & 160 \\
\hline $\mathrm{DH} 3 \mathrm{X}$ & 4035510980135 & T7N R06W 08 & 3 & $\begin{array}{l}\text { Sprinkler irrigated cropland, bottom- } \\
\text { land, continuous corn }\end{array}$ & 180 & 180 \\
\hline DH3Y & 4035560980135 & T7N R06W 08 & 3 & $\begin{array}{l}\text { Sprinkler-irrigated cropland, upland, } \\
\text { continuous corn }\end{array}$ & 180 & 180 \\
\hline $\mathrm{DH} 4 \mathrm{X}$ & 4105100974616 & T13N R04W 21 & 4 & $\begin{array}{l}\text { Nonirrigated cropland, upland, } \\
\text { conventional tillage, continuous corn }\end{array}$ & 120 & 120 \\
\hline DH4Y & 4105140974624 & T13N R04W 21 & 4 & $\begin{array}{l}\text { Nonirrigated pasture land, bottomland, } \\
\text { horse track }\end{array}$ & 0 & 0 \\
\hline $\mathrm{DH} 4 \mathrm{Z}$ & 4105280974625 & T13N R04W 21 & 4 & $\begin{array}{l}\text { Gravity-irrigated cropland, bottomland, } \\
\text { conventional tillage, corn ( } 2 \text { years) } \\
\text { and bean ( } 1 \text { year) rotation }\end{array}$ & 190 & 190 \\
\hline DH5 & 4055010973818 & T11N R03W 15 & 5 & $\begin{array}{l}\text { Gravity-irrigated cropland, upland, ridge } \\
\text { till, continuous corn, }\end{array}$ & 190 & 190 \\
\hline DH6X & 4036030973402 & T07N R02W 5 & 6 & Pasture land, upland & 0 & 0 \\
\hline DH6Y & 4035490973433 & T07N R02W 5 & 6 & $\begin{array}{l}\text { Gravity irrigated cropland, upland, } \\
\text { conventional tillage, continuous corn }\end{array}$ & 180 & 180 \\
\hline DH7X & 4105000971555 & T10N R03E 24 & 7 & $\begin{array}{l}\text { Gravity-irrigated cropland, bottomland, } \\
\text { minimum tillage, corn ( } 2 \text { years) and } \\
\text { bean ( } 1 \text { year) rotation }\end{array}$ & 160 & 0 \\
\hline $\mathrm{DH} 7 \mathrm{Y}$ & 4104580971602 & T10N R03E 24 & 7 & $\begin{array}{l}\text { Nonirrigated cropland, bottomland, } \\
\text { minimum tillage, sorghum }\end{array}$ & 120 & 120 \\
\hline DH8 & 4049330971729 & T10N R01E 22 & 8 & $\begin{array}{l}\text { Sprinkler irrigated cropland, upland, } \\
\text { ridge till, corn and soybean rotation }\end{array}$ & 0 & 180 \\
\hline
\end{tabular}

filter. Samples collected for analysis of dissolved nitrate were chilled to $4{ }^{\circ} \mathrm{C}$ immediately after sample collection, and this temperature was maintained during transport to the laboratory. The samples collected for analysis of major ions were preserved in accordance with methods described by Pritt and Jones (1989). Water samples for tritium analysis were collected in a 1-L (liter) plastic bottle, and were not filtered, chilled, or preserved.

\section{Unsaturated-Zone Samples}

Two-hundred and fifty sediment samples were collected from 1995 through 1997 and analyzed for nitrate content. In November and December 1995, cores were collected to a depth equal to the water table (or perched water table at site 3 ), of which the total depth varied from 15 to $90 \mathrm{ft}$. The cores were collected with a split-spoon sampler by a 
contractor with oversight from USGS and UBBNRD personnel. From December 1996 through January 1997 and in March 1997, samples were collected with a probe by UBBNRD personnel with oversight by USGS personnel. In general, samples were collected every $5 \mathrm{ft}$ or at each lithologic change to a depth of $20 \mathrm{ft}$. At greater depths up to $31 \mathrm{ft}$, samples were collected about every $10 \mathrm{ft}$, or when a lithologic change warranted another sample. Sediment samples were kept below $4{ }^{\circ} \mathrm{C}$ to limit microbial degradation and chemical reactions.

\section{Laboratory Procedures}

Standard analytical procedures were used for laboratory analyses. Water samples to be analyzed for dissolved nitrate and alkalinity, residue on evaporation at $180^{\circ} \mathrm{C}$, and major ions were sent to the USGS National Water-Quality Laboratory (NWQL) in Arvada, Colorado, and analyzed in accordance with standard procedures described by Fishman and Friedman (1989). The analyses for tritium were done using an electrolytic enrichment gas-counting method at the University of Miami in Florida.

Unsaturated-zone sediment samples were analyzed for total nitrate by the Soil Testing Laboratory of the University of Nebraska-Lincoln (Page and others, 1982, p. 679-682). Other nitrogen species were not analyzed because it was assumed that concentrations of other nitrogen species would be negligible.

\section{Data-Analysis Procedures}

Statistical analyses were performed on the data to determine the distributions of variables, to identify relations between variables, and to test the significance of these relations. Analyses of data from the monitoring network and nested wells included the use of general statistical analyses, boxplots, the Spearman rank correlation coefficient, the ShapiroWilk test, the Kruskal-Wallis or Wilcoxon rank-sum test, and, when more than two groupings existed, Tukey's multiple comparison test (Helsel and Hirsch, 1992; Verstraeten and Ellis, 1995). A confidence level of 95 percent generally was used for interpretation. This means that the interpretation can be wrong in 5 of 100 cases.

The Shapiro-Wilk test was used to check for normality of the data sets and showed that field measurements and nitrate concentrations of water samples from the monitoring network are not normally distributed. Therefore, nonparametric statistical analyses that are not sensitive to outlying values were used for statistical interpretation. Either the Kruskal-Wallis (multiple groups) or Wilcoxon rank-sum (two groups) test was used to determine whether the median value of a constituent was statistically different among or between groups. Tukey's test was used to perform multiple comparisons to find which groups had statistically differing medians. The data were grouped to test for differences among and within groups according to:

(1) aquifer; (2) hydrogeologic condition; and

(3) management zone.

\section{Quality Control and Quality Assurance}

The chance of unrecognized contamination of the water-quality samples, including from bottles and preservatives, was reduced by quality control (QC) measures both in the field and in the laboratory. During sampling of the monitoring network, 29 duplicate water samples and 31 equipment blanks were collected. One duplicate and one equipmentblank sample were collected from well 8A (at nested-well site 8) 10 of the 13 times the nested wells were sampled. At one unsaturated-zone sample collection subsite, duplicate samples were collected from a nearby site (less than $50 \mathrm{ft}$ ) with the same land and chemical use and on the same landscape position.

At the NWQL, the QA program includes participation in the USGS and U.S. Environmental Protection Agency (USEPA) interlaboratory evaluations and submission of blind standard reference water samples to the USGS laboratory sample stream (Fishman and Friedman, 1989; Jones, 1987). In addition, cation-anion balances were calculated for each major-ion analysis. The cationanion balances were within 10 percent for all samples analyzed for this study. 
Nitrate concentrations in equipment blanks from the monitoring network varied from less than the method reporting level of 0.05 to $0.16 \mathrm{mg} / \mathrm{L}$ (milligrams per liter) with concentrations of 17 of the 29 equipment blanks below the method reporting level. Dissolved nitrate concentrations in equipment blanks from well 8A (well-numbering system is discussed under the Monitoring Approach Section) varied from less than the method reporting level of 0.05 to $0.07 \mathrm{mg} / \mathrm{L}$. Seven of the 15 equipment blanks from well $8 \mathrm{~A}$ had nitrate concentrations less than the method reporting level. The median nitrate concentration in all of the equipment blanks was $0.05 \mathrm{mg} / \mathrm{L}$, with 75 percent of the samples having concentrations less than $0.07 \mathrm{mg} / \mathrm{L}$. Concentrations of nitrate in duplicate samples collected from the monitoring network and well nests generally were within 10 percent of the nitrate concentrations in the environmental sample. The maximum difference in environmental and duplicate sample nitrate concentrations in the monitoring network was $0.6 \mathrm{mg} / \mathrm{L}$. The Wilcoxon rank-sum test showed no significant differences in nitrate concentrations between the environmental and duplicate samples from the monitoring network (the two-sided p-value is 0.88) and those from the well nests (the two-sided $\mathrm{p}$-value is 0.37 ). A p-value is the probability of obtaining a test statistic equal to or more extreme than the value computed from the same data when the medians of the two populations being tested are the same. Using a 95-percent confidence interval, pvalues of 0.05 or smaller indicate that the medians of the populations being considered are significantly different. The smaller the p-value, the greater is the confidence in that difference.

In addition, split samples from the seven water samples collected in 1997 were sent to the NWQL and the Grand Island/Hall County Department of Health Laboratory in Grand Island, Nebraska, the laboratory which normally analyzes water samples for nitrates for the UBBNRD (table 7). The Wilcoxon rank-sum test shows that the concentrations of nitrate did not vary significantly between split samples (the two-sided p-value is 0.96).
Table 7. Results of split-sample analysis by the USGS National Water Quality Laboratory (sample 1) and the Grand Island Hall County Department of Health Laboratory (sample 2)

[Data provided by R.D. DeBuhr, Upper Big Blue Natural Resources District, written commun., 1998]

\begin{tabular}{ccc}
\hline $\begin{array}{c}\text { Well identification } \\
\text { number } \\
\text { (fig. 7) }\end{array}$ & \multicolumn{2}{c}{$\begin{array}{c}\text { Nitrate concentration } \\
\text { (milligrams per liter) }\end{array}$} \\
\cline { 2 - 3 } 0321 & Sample 1 & Sample 2 \\
0706 & 6.2 & 5.7 \\
0717 & .16 & .30 \\
0809 & 6.8 & 6.7 \\
0902 & 10 & 9.7 \\
1205 & .31 & .70 \\
1212 & 15 & 16 \\
\hline
\end{tabular}

Nitrate concentrations varied widely from 1995 to 1996 in water samples from some wells of the proposed ground-water-quality monitoring network. Water samples from 13 of the 417 wells that were sampled in both 1995 and 1996 had large variations in nitrate concentrations (data are flagged in the list of results in table 8). These wells were scheduled for resampling in 1997. Water samples from only nine of these wells could be collected because of weather and other logistical reasons. An additional well containing water with unexplainable large nitrate concentrations in 1996 was resampled in 1997. The Wilcoxon rank-sum test performed on the samples collected in 1995, 1996, and 1997 shows no difference in median nitrate concentrations among the samples collected in 1995 and 1997 (the two-sided p-value is 0.59). Significant differences existed between the samples collected in 1995 and those collected in 1996 (the two-sided P-value is $0.03)$.

The reason for the wide variability in results is unknown. Field contamination was omitted as a potential source of variability, because the variability of nitrate concentrations exceeded the highest detection of nitrate contained in the blank samples. Laboratory recording error was explored and samples were re-analyzed when possible. No analytical errors of more than $1 \mathrm{mg} / \mathrm{L}$ were identified. Therefore, the wells showing large 
variability in nitrate concentrations were not omitted from the sampling program because of the large variability alone. The Wilcoxon rank-sum test on the duplicate samples collected from the unsaturated zone of two nearby subsites with similar land and chemical use showed that there was no significant difference in the total nitrate concentrations between environmental and duplicate samples (the two-sided $\mathrm{p}$-value is 0.93).

\section{TRENDS IN NITRATE CONCENTRA- TIONS, FIELD MEASUREMENTS, AND MAJOR-ION AND TRITIUM CONCENTRATIONS IN GROUND WATER}

This section addresses the ground-water quality in the UBBNRD with the focus on spatial and temporal distribution of nitrate concentrations in ground water. The water-quality data collected for this study are included in table 8 at the back of this report and are stored in the USGS's National Water Information System (NWIS) database.

\section{Spatial Trends in Nitrate Concentra- tions and Field Measurements in Ground Water}

\section{Ground-Water Monitoring Network}

Water samples were collected from 206 wells in 1995, 211 wells in 1996, and 10 wells in 1997. Only wells retained in the ground-water-quality monitoring network are shown on figure 8, 9, 10, and 11. A statistical summary of the 1995 (selected data) and 1996 analytical data collected from wells retained as part of the ground-water monitoring network by (1) aquifer, (2) hydrogeologic condition, and (3) management zone is listed in table 9 (at back of report) and boxplots are illustrated in figures 9 through 11. If no water samples were collected from a well of the ground-water-quality monitoring network in 1996, the data collected in 1995 were used in the statistical analyses. Well 1001, completed in the Greenhorn Formation, was considered to be completed in the Dakota aquifer for statistical purposes and interpretation.
Nitrate concentrations in water samples collected from the ground-water-quality monitoring network varied from 0.06 to $27 \mathrm{mg} / \mathrm{L}$, with a median concentration of $4.6 \mathrm{mg} / \mathrm{L}$ (table 9 ). In the shallow part of the High Plains aquifer, the concentrations varied from 0.09 to $27 \mathrm{mg} / \mathrm{L}$, with a median concentration of $6.3 \mathrm{mg} / \mathrm{L}$ (fig. 9 and table 9). In the deep part of the High Plains aquifer, the concentrations were generally less than in the shallow part. Nitrate concentrations varied from 0.06 to $21 \mathrm{mg} / \mathrm{L}$ with a median concentration of $3.4 \mathrm{mg} / \mathrm{L}$. In the Dakota aquifer, the concentrations varied between 0.08 and $0.76 \mathrm{mg} / \mathrm{L}$, with a median concentration of $0.11 \mathrm{mg} / \mathrm{L}$.

Of the 197 water samples collected in 1995 and 1996, 41 water samples (21 percent), exceeded the USEPA Maximum Contaminant Level (MCL) for drinking water of $10 \mathrm{mg} / \mathrm{L}$ for nitrate as nitrogen (U.S. Environmental Protection Agency, 1996). Thirty-one of these water samples were obtained from wells completed in the unconfined part of the High Plains aquifer.

Nitrate concentrations of water samples collected from unconfined (median was $6.2 \mathrm{mg} / \mathrm{L}$ ) and confined conditions (median was $2.9 \mathrm{mg} / \mathrm{L}$ ) (fig. 10 and table 9) were similar to those classified as water samples collected from shallow and deep parts of the High Plains aquifer, respectively. These results suggest that, assuming that the water samples collected from the ground-water-quality monitoring network are representative of the conditions in the High Plains aquifer in the study area: (1) more than 50 percent of the shallow or unconfined part of the High Plains aquifer in the study area may contain ground water with nitrate concentrations that exceed $6 \mathrm{mg} / \mathrm{L}$; and, (2) more than 25 percent of the shallow part of the High Plains aquifer in the study area may contain ground water with nitrate concentrations that exceed the action level (Phase II trigger) of $9 \mathrm{mg} / \mathrm{L}$ established by the UBBNRD's Special Protection Area action plan (Upper Big Blue Natural Resources District, 1994). In contrast, nitrate concentrations in more than 75 percent of the deep or confined part of the High Plains aquifer appear to be less than $6 \mathrm{mg} / \mathrm{L}$; nitrate concentrations in more than 50 percent of these parts of the High Plains aquifer appear to be less than $3.5 \mathrm{mg} / \mathrm{L}$. 


\section{EXPLANATION}

Registered well location and identification

number (table 4) with color illustrating nitrate

concentration, as nitrogen, in milligrams per liter

Unconfined part of the High Plains aquifer

$\Delta$ Less than 0.05 to 4.0

A More than 4.0 to 9.0

More than 9.0 to 12

A More than 12

Confined part of the High Plains aquifer, Greenhorn Formation or Dakota aquifer

Less than 0.05 to 4.0

- More than 4.0 to 9.0

Int More than 9.0 to 12

More than 12

12

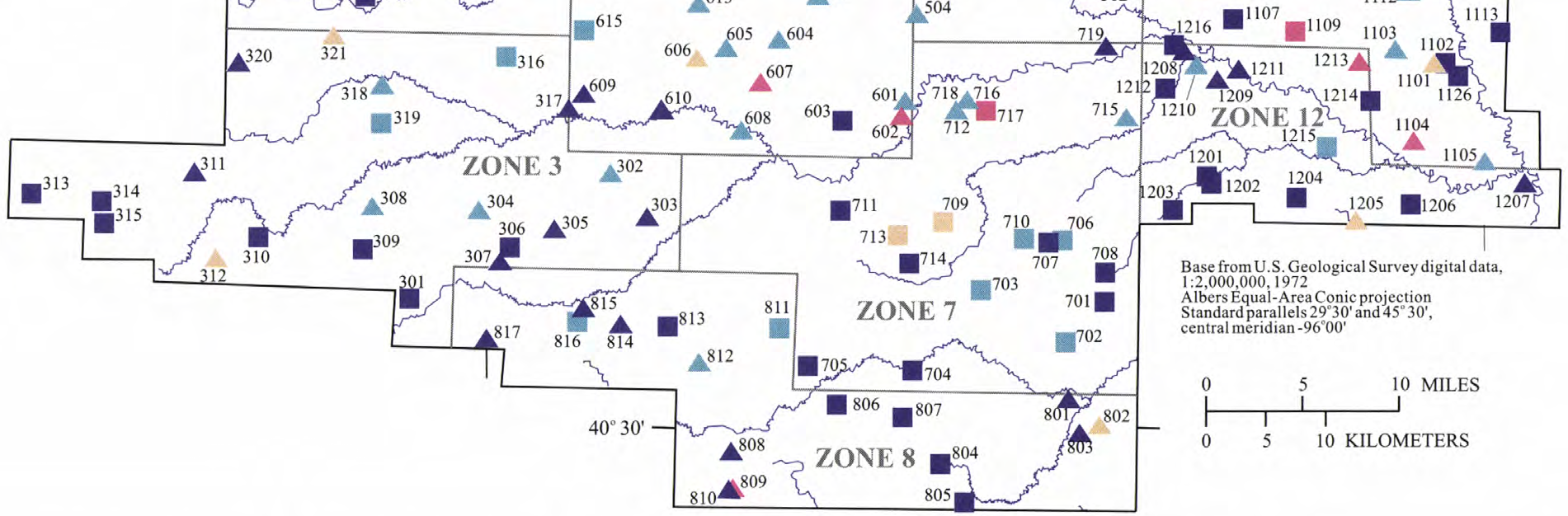

Figure 8. Nitrate concentrations and hydrogeologic conditions of the ground-water-quality monitoring network, Upper Big Blue Natural Resources District, central Nebraska, July 1995 through September 1996. 


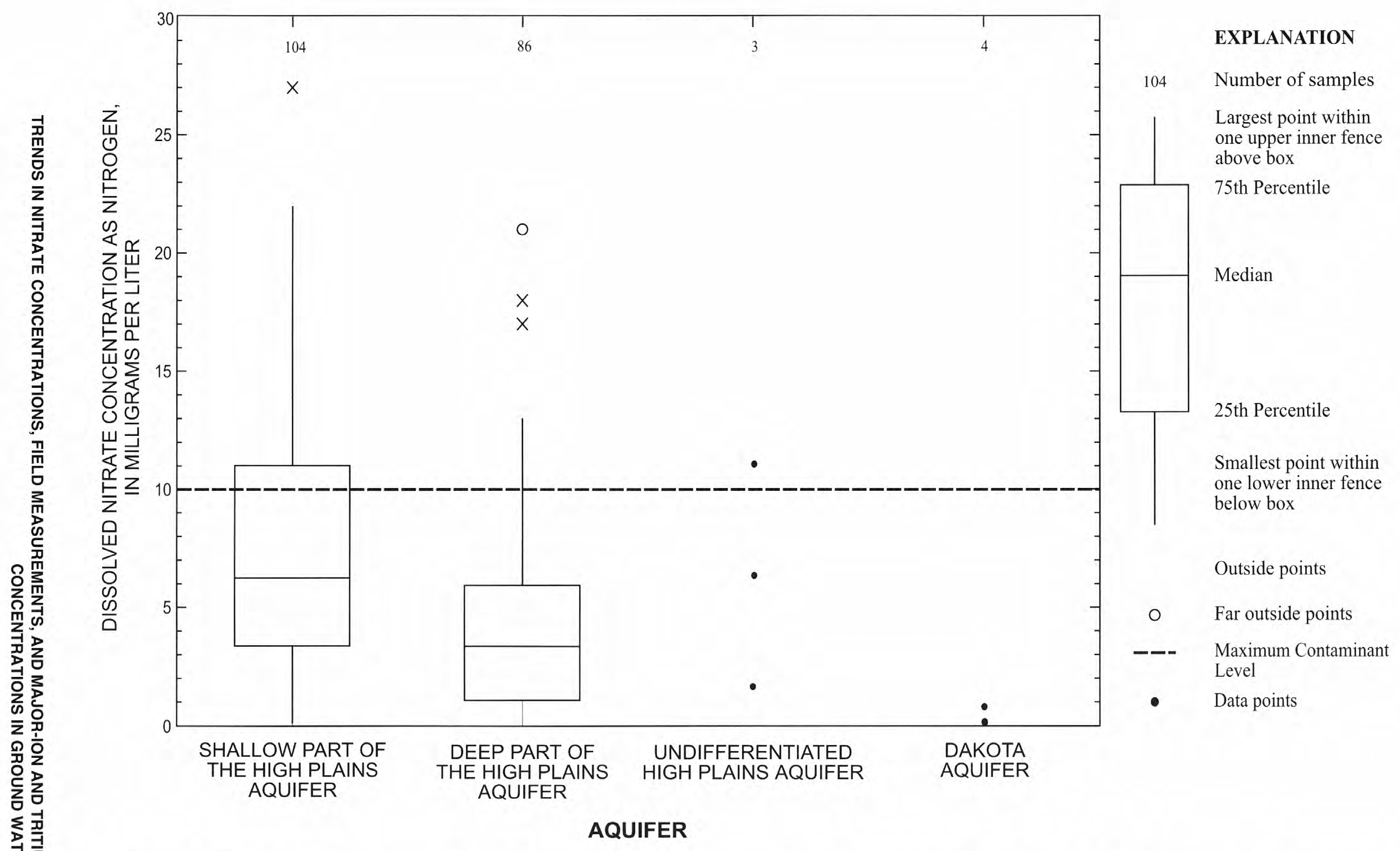

Figure 9. Distribution of nitrate concentrations by aquifer in the ground-water-quality monitoring network, Upper Big Blue Natural Resources District, central Nebraska, July 1995 through September 1996. 

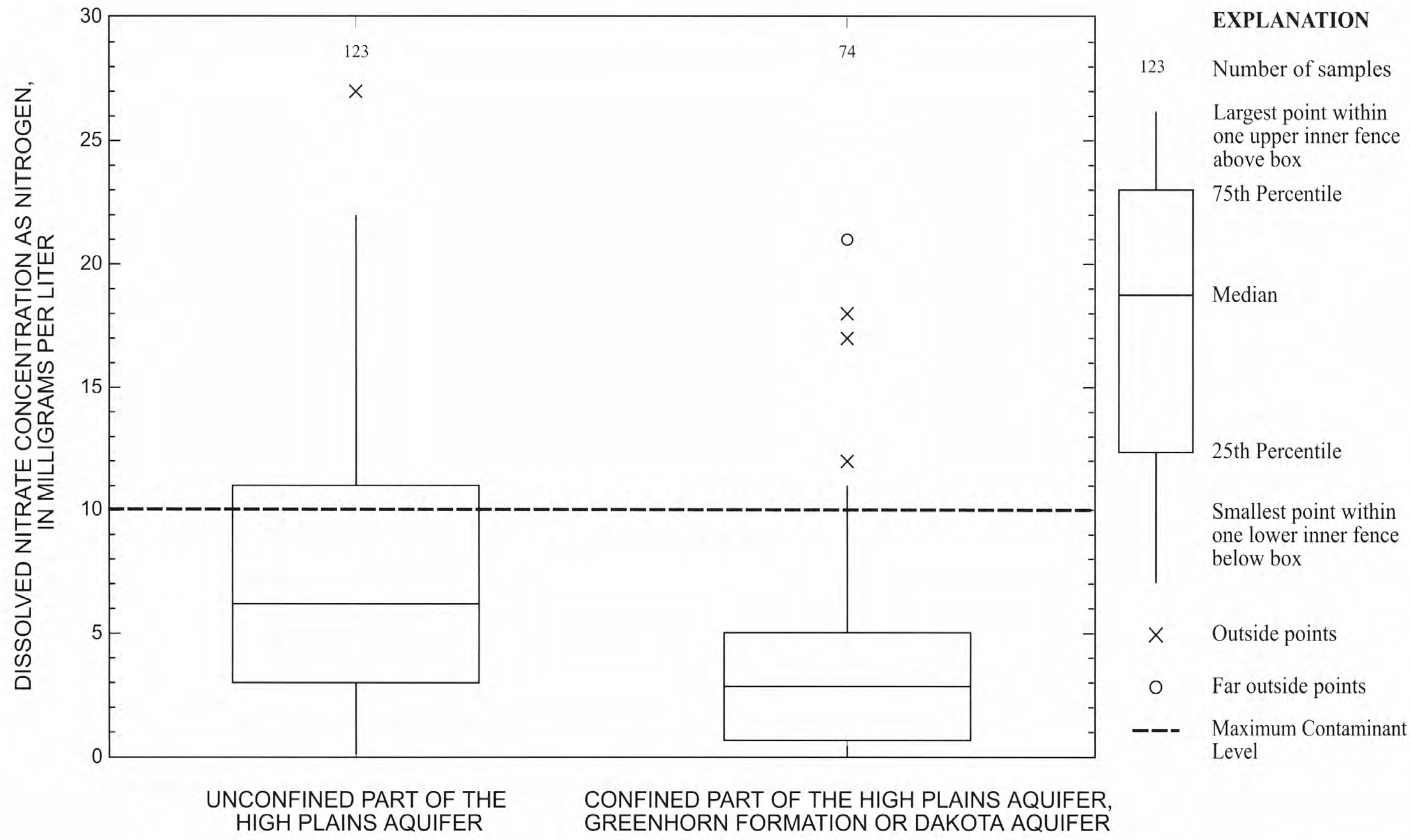

\section{HYDROGEOLOGIC CONDITION}

Figure 10. Distribution of nitrate concentrations by hydrogeologic condition in the ground-water-quality monitoring network, Upper Big Blue Natural Resources District, central Nebraska, July 1995 through September 1996. 


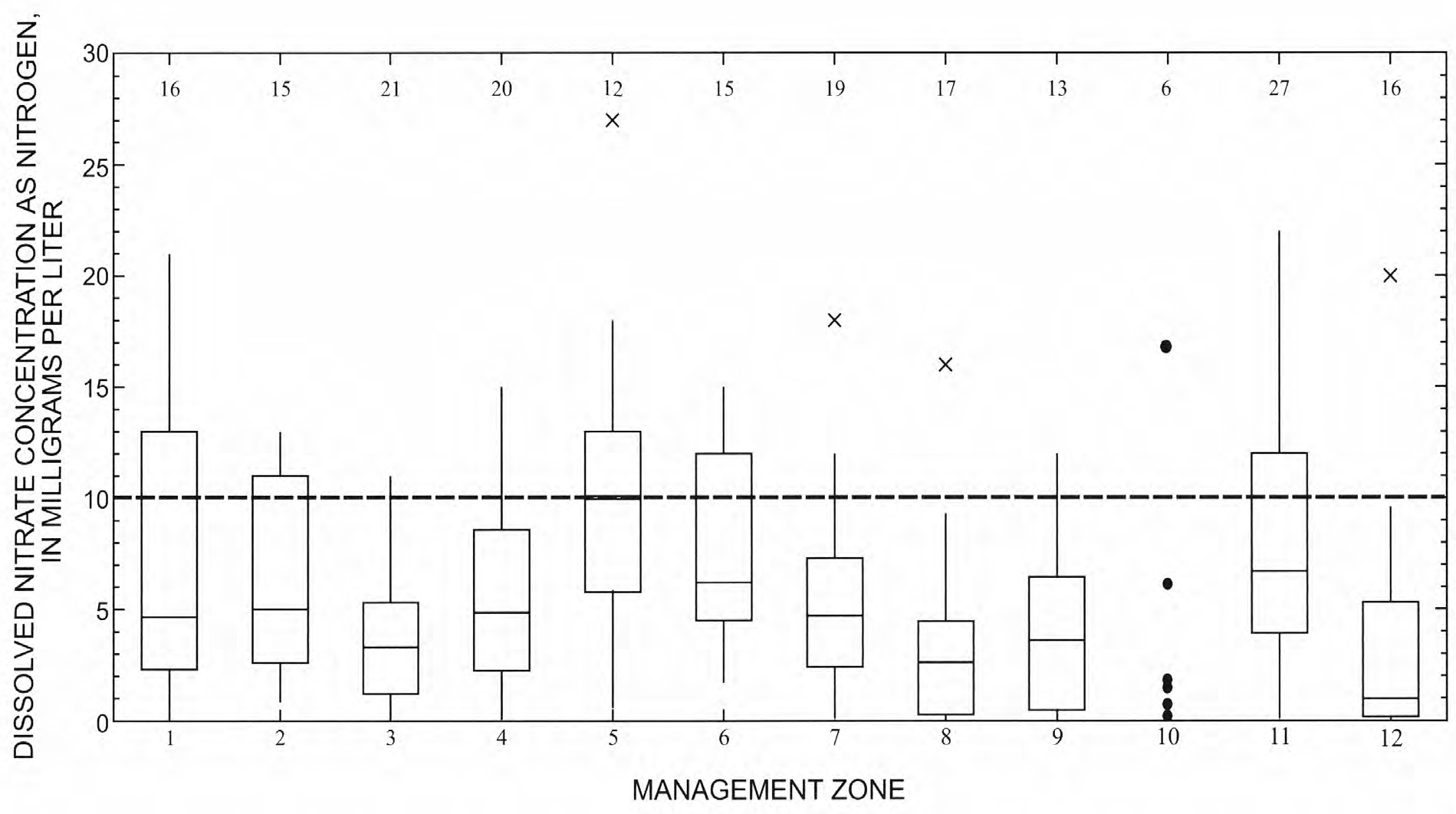

Number of samples

Largest point within one upper inner fence above box

\section{5th Percentile}

\begin{tabular}{|l|l}
\hline & Median \\
& 25 th Percentile \\
$\begin{array}{l}\text { Smallest point within } \\
\text { one lower inner fence }\end{array}$
\end{tabular} one lower inner fence below box

$\times \quad$ Outside points

--- Maximum Contaminant Level

- Data point

Figure 11. Distribution of nitrate concentrations by management zone in the ground-water-quality monitoring network, Upper Big Blue Natural Resources District central Nebraska, July 1995 through September 1996. 
Water samples from 10 of 74 wells screened in the confined part of the High Plains aquifer had concentrations exceeding the USEPA MCL for drinking water of $10 \mathrm{mg} / \mathrm{L}$ (U.S. Environmental Protection Agency, 1996). In numerous cases, the annulus between the casing and the borehole wall are filled with gravel only and an intervening screen between the aquifer and land surface was not installed or has deteriorated, allowing entry of surface contaminants into the ground water. In other cases, the retarding clay layer may be discontinuous nearby, or nearby wells may be screened in both shallow and deep parts of the High Plains aquifer, leading to cross-contamination.

Because there often is no way to distinguish definitively between nonpoint and point sources of ground-water contamination with nitrate, it is difficult to exclude all wells affected by point sources of contamination or by cross-contamination of unconfined and confined aquifers in a network of monitoring wells. To reduce the influence of water samples affected by point sources, the median, and the 25th and 75th percentiles can be used to reflect the majority of the samples, and to aid management decisions. However, large nitrate concentrations caused by nonpoint-source contamination have been observed in other parts of Nebraska, such as in northern Holt County (Marty Link, Department of Environmental Quality, written commun., 1998).

Among the 12 management zones, the median nitrate concentrations varied from 0.97 to $10 \mathrm{mg} / \mathrm{L}$ (fig. 11 and table 9). The median nitrate concentrations (in $\mathrm{mg} / \mathrm{L}$ ) by zone ranked from smallest to largest were as follows: 0.97 in zone 12 , 1.6 in zone 10, 2.6 in zone 8, 3.3 in zone $3,3.6$ in zone $9,4.7$ in zones 1 and 7, 4.9 in zone $4,5.0$ in zone 2, 6.2 in zone $6,6.7$ in zone 11 , and 10 in zone 5 . Median nitrate concentrations in water samples collected from wells located in zone 5 exceeded the action level of $9 \mathrm{mg} / \mathrm{L}$ established in the UBBNRD's Special Protection Area Action Plan (Upper Big Blue Natural Resources District, 1994).

Nonparametric statistical analyses using the Kruskal-Wallis test (Helsel and Hirsch, 1992) on the water-quality data leads to numerous conclusions. Nitrate concentration (two-sided p-value $=0.00,197$ samples), dissolved oxygen (two-sided $\mathrm{p}$-value $=0.00,172$ samples), and $\mathrm{pH}$ (two-sided $\mathrm{p}$-value $=0.00,195$ samples) differed significantly depending upon the sources of water being compared. The nitrate concentrations in water from the Dakota aquifer and water from the deep part of the High Plains aquifer are significantly smaller than in water from the shallow part of the High Plains aquifer (table 10).

Median nitrate concentrations (two-sided p-value $=0.00,197$ samples), dissolved oxygen (two-sided p-value $=0.00,172$ samples), and $\mathrm{pH}$ (two-sided $\mathrm{p}$-value $=0.00,195$ samples) differed significantly among the aquifer groups depending upon the hydrogeologic condition of the aquifer from which the water was derived. The nitrate and dissolved oxygen concentrations tended to be largest in the unconfined part of the High Plains aquifer, generally suggesting the possible absence of denitrifying conditions.

Median values for well depth (two-sided p-value $=0.00,196$ samples $)$, nitrate concentration (two-sided p-value $=0.00,197$ samples), dissolved oxygen (two-sided $\mathrm{p}$-value $=0.00,172$ samples $)$, and $\mathrm{pH}$ (two-sided $\mathrm{p}$-value $=0.00,195$ samples) in the ground water varied among management zones. The Tukey's test (Helsel and Hirsch, 1992) indicates generally smaller nitrate concentrations in ground water in zones 12 and 8 and larger nitrate concentrations in zone 11,6 , and, especially 5 (table 9 and table 11).

Nitrate concentrations (two-sided p-value $=0.00,197$ samples), $\mathrm{pH}$ (two-sided p-value $=0.00$, 194 samples), and dissolved oxygen concentrations (two-sided p-value $=0.00,171$ samples) in the ground water varied by depth (fig. 12 and table 12). Nonparametric statistical analyses established no relation between nitrate concentration and landscape position, depth to water (R.D. DeBuhr, Upper Big Blue Natural Resources District, written commun., 1998), or soil drainage class.

Spearman rank correlation coefficients (P) (Helsel and Hirsch, 1992) (99-percent confidence level, 159 samples) suggest the following relations:

a. Concentrations of nitrate in the ground water are larger in the shallow aquifers than in the deep aquifers $(\mathrm{P}=-0.33)$; 
Table 10. Results of Tukey's test (95-percent confidence level) for differences in nitrate concentrations among aquifers, Upper Big Blue Natural Resources District, central Nebraska

[mg/L, milligrams per liter]

\begin{tabular}{|c|c|c|c|c|}
\hline $\begin{array}{c}\text { Aquifer } \\
\text { (Median nitrate } \\
\text { concentration in } \mathrm{mg} / \mathrm{L})\end{array}$ & $\begin{array}{c}\text { Dakota aquifer } \\
\mathbf{( 0 . 1 1 )}\end{array}$ & $\begin{array}{c}\text { Deep part of the High } \\
\text { Plains aquifer } \\
\mathbf{( 3 . 4 )}\end{array}$ & $\begin{array}{c}\text { Undifferentiated High } \\
\text { Plains aquifer } \\
(\mathbf{6 . 4 )}\end{array}$ & $\begin{array}{c}\text { Shallow part of the } \\
\text { High Plains aquifer } \\
\mathbf{( 6 . 3 )}\end{array}$ \\
\hline $\begin{array}{c}\text { Significantly different } \\
\text { groups of data }\end{array}$ & & & & \\
\cline { 2 - 5 } & & & & \\
\hline
\end{tabular}

${ }^{1}$ Groups shaded along the same row are not significantly different.

Table 11. Results of Tukey's test (95-percent confidence level) for differences in nitrate concentrations among management zones, Upper Big Blue Natural Resources District, central Nebraska

[mg/L, milligrams per liter]

\begin{tabular}{|c|c|c|c|c|c|c|c|c|c|c|c|c|}
\hline $\begin{array}{l}\text { Management zone } \\
\text { (Median nitrate } \\
\text { concentration in } \mathrm{mg} / \mathrm{L} \text { ) }\end{array}$ & $\begin{array}{c}12 \\
(0.97)\end{array}$ & $\begin{array}{c}8 \\
(2.6)\end{array}$ & $\begin{array}{c}10 \\
(1.6)\end{array}$ & $\begin{array}{c}9 \\
(3.6)\end{array}$ & $\begin{array}{c}3 \\
(3.3)\end{array}$ & $\begin{array}{c}7 \\
(4.7)\end{array}$ & $\begin{array}{c}4 \\
(4.9)\end{array}$ & $\begin{array}{c}2 \\
(5.0)\end{array}$ & $\begin{array}{c}1 \\
(4.7)\end{array}$ & $\begin{array}{c}11 \\
(6.7)\end{array}$ & $\begin{array}{c}6 \\
(6.2)\end{array}$ & $\begin{array}{c}5 \\
(10)\end{array}$ \\
\hline \multirow{2}{*}{\multicolumn{13}{|c|}{$\begin{array}{l}\text { Significantly different } \\
\text { groups of data }{ }^{1}\end{array}$}} \\
\hline & & & & & & & & & & & & \\
\hline & & & & & & & & & & & & \\
\hline & & & & & & & & & & & & \\
\hline
\end{tabular}

${ }^{1}$ Groups shaded along the same row are not significantly different.

Table 12. Results of Tukey's test (95-percent confidence level) for differences in nitrate concentrations among well depths, Upper Big Blue Natural Resources District, central Nebraska

[mg/L, milligrams per liter]

\begin{tabular}{|c|c|c|c|c|c|}
\hline $\begin{array}{c}\text { Well depth } \\
\text { (Median nitrate concentration } \\
\text { in } \mathrm{mg} / \mathrm{L} \text { ) }\end{array}$ & $\begin{array}{l}\text { More than } \\
300 \text { feet } \\
(1.1)\end{array}$ & $\begin{array}{c}\text { From } 226 \text { to } \\
300 \text { feet } \\
\text { (3.9) }\end{array}$ & $\begin{array}{c}\text { From } 151 \text { to } \\
225 \text { feet } \\
(4.6)\end{array}$ & $\begin{array}{c}\text { From } 0 \text { to } 75 \\
\text { feet } \\
(4.6)\end{array}$ & $\begin{array}{c}\text { From } 76 \text { to } \\
150 \text { feet } \\
(6.8)\end{array}$ \\
\hline Significantly different groups of data ${ }^{1}$ & & & & & \\
\hline
\end{tabular}

${ }^{1}$ Groups shaded along the same row are not significantly different.

b. Concentrations of nitrate are larger in ground water under unconfined conditions $(\mathrm{P}=-0.37)$;

c. Concentrations of nitrate increase as the well depth decreases $(\mathrm{P}=-0.27)$;

d. Concentrations of nitrate in ground water increase as the amount of oxygen in ground water increases $(\mathrm{P}=0.61)$;

e. Concentrations of nitrate increase as the specific conductance of the water increases $(\mathrm{P}=0.40)$;

f. Amount of dissolved oxygen in the ground water is larger in the shallow or unconfined part of the High Plains aquifer than in the deep or confined part of the High Plains aquifer $(\mathrm{P}=-0.28$ or -0.38$)$;

g. Amount of dissolved oxygen decreases with depth of well $(\mathrm{P}=-0.38)$;

h. The temperature increases with depth of well $(P=0.26)$; and,

i. $\mathrm{pH}$ tends to increase with the depth of the well $(\mathrm{P}=0.29)$.

The strongest correlation indicated by the Spearman rank correlation coefficients was between nitrate concentration and the amount of dissolved oxygen. The specific conductance of ground water in the UBBNRD apparently does not vary significantly by aquifer, hydrogeologic condition, or management zone. 


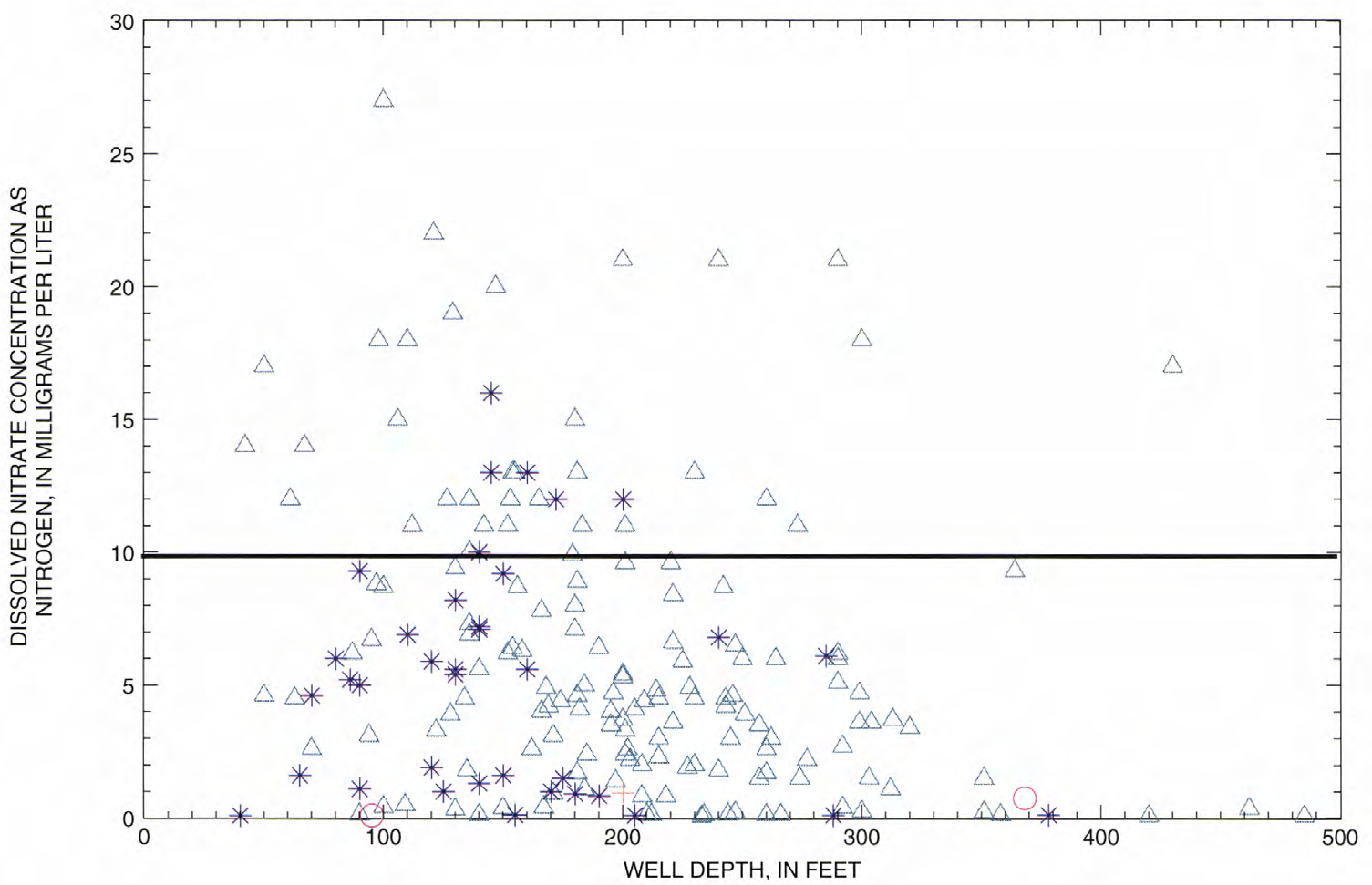

\section{EXPLANATION}

$\triangle$ Irrigation well

* Domestic well

C Municipal well

+ Industrial well

Maximum contaminant

level

Figure 12. Distribution of nitrate concentrations by well depth in the ground-water-quality monitoring network, Upper Big Blue Natural Resources District, central Nebraska, July 1995 through September 1996. 


\section{Nested Wells}

Two hundred thirty-two water samples were collected from the nested wells by UBBNRD personnel (fig. 13; table 13 at back of report). Water samples were collected monthly from November 1995 to July 1996 and bimonthly from September 1996 to May 1997. Water levels generally were measured before sample collection. A statistical summary of the nitrate concentrations is listed in table 14 (at back of report).

The data collected from the nested wells (tables 13 and 14) generally support findings discussed for the data collected from the groundwater-quality monitoring network of domestic and registered wells. Water collected from the shallow part of the High Plains aquifer had a median nitrate concentration of $9.2 \mathrm{mg} / \mathrm{L}$ (table 14). Water collected from the deep part of the High Plains aquifer had a median nitrate concentration of 0.42 $\mathrm{mg} / \mathrm{L}$. Water collected from the unconfined and confined parts of the High Plains aquifer had median concentrations of 7.1 and $0.10 \mathrm{mg} / \mathrm{L}$, respectively (table 14).

Water collected from well 7A generally had smaller nitrate concentrations than water collected from other nested wells completed in the unconfined shallow part of the High Plains aquifer (fig. 13). Additional data are needed to better understand the reason for the small nitrate concentrations in water obtained from this well. Dissolved-oxygen concentrations from 1.0 to $8.0 \mathrm{mg} / \mathrm{L}$ were present in the water collected at this site (table 13).

Nitrate concentrations in water samples from well 8B, screened from a depth of 120 to $130 \mathrm{ft}$, were larger than concentrations in water samples from well $8 \mathrm{~A}$, screened from a depth of 90 to $100 \mathrm{ft}$ (fig. 13 and table 5). The dissolved-oxygen concentrations of water samples collected from wells $8 \mathrm{~A}$ and $8 \mathrm{~B}$ were similar, varying from 5.4 to $10.2 \mathrm{mg} / \mathrm{L}$ (table 13), and are too large to support denitrifying conditions. Potential explanations would be that, at shallow depths, (1) a dilution effect is occurring or (2) differing ground-water flow paths and time of travel exist.

Water samples with nitrate concentrations that exceeded the USEPA MCL of $10 \mathrm{mg} / \mathrm{L}$ for drinking water (U.S. Environmental Protection Agency, 1996) were collected from wells $1 \mathrm{~A}, 4 \mathrm{~A}, 8 \mathrm{~A}$, and 8B screened in the shallow part of the High Plains aquifer. The Upper Big Blue Special Protection Area Action Plan Phase III trigger of $12 \mathrm{mg} / \mathrm{L}$ of nitrate was exceeded in wells $1 \mathrm{~A}, 4 \mathrm{~A}, 8 \mathrm{~A}$, and $8 \mathrm{~B}$. The largest nitrate concentrations generally were detected in water samples from the unconfined part of the High Plains aquifer. Intermediate nitrate concentrations generally were detected in water samples from the unconfined deep part of the High Plains aquifer. The smallest nitrate concentrations generally were detected in water samples from the confined part of the High Plains aquifer.

Statistical analyses (Kruskal-Wallis test) suggest significant differences in nitrate concentrations by site, by aquifer, and by hydrogeologic condition (two-sided p-value $=0.00$; 229 samples). In addition, Spearman rank correlation coefficients (99-percent confidence level; 205 samples) support the relation of the nitrate concentrations and field measurements established with the data from the ground-water-quality monitoring network. For example, water under unconfined conditions has larger nitrate concentrations and dissolved oxygen than water under confined conditions.

The shallow part of the High Plains aquifer appears to be susceptible to contamination with large nitrate concentrations. The potential for large nitrate concentrations in the High Plains aquifer is of most concern where (1) the shallow part of the High Plains aquifer is thin and in areas where confining conditions are absent, such as in parts of Seward and York Counties, or (2) where cross-contamination of unconfined and confined parts of the High Plains aquifer occurs, such as in York County. 
Well nest 1

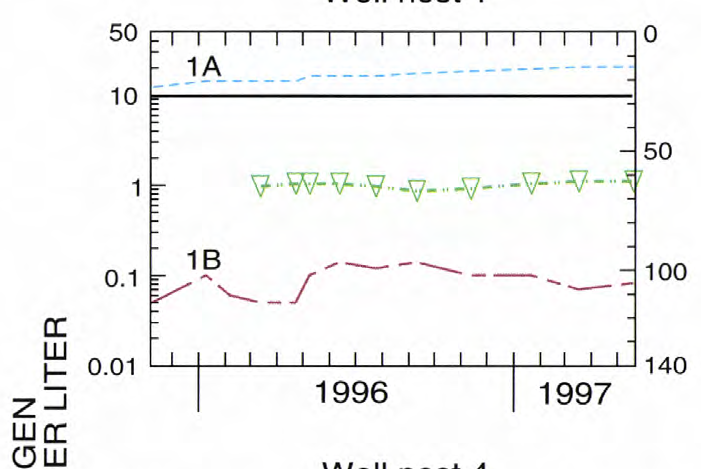

Well nest 4

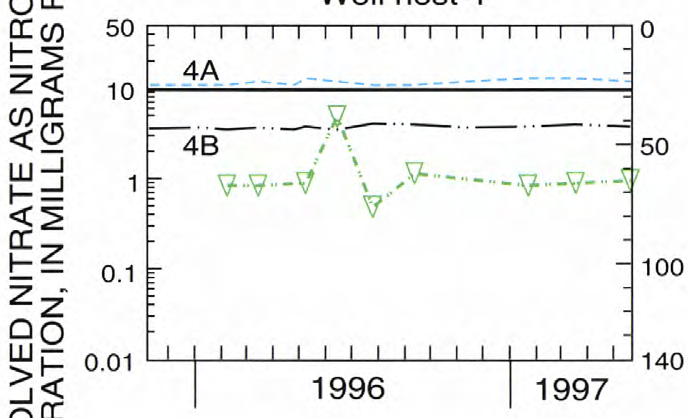

Well nest 7
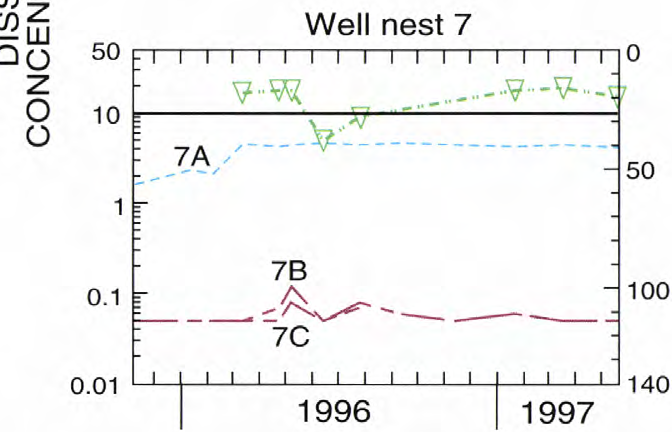

Well nest 2

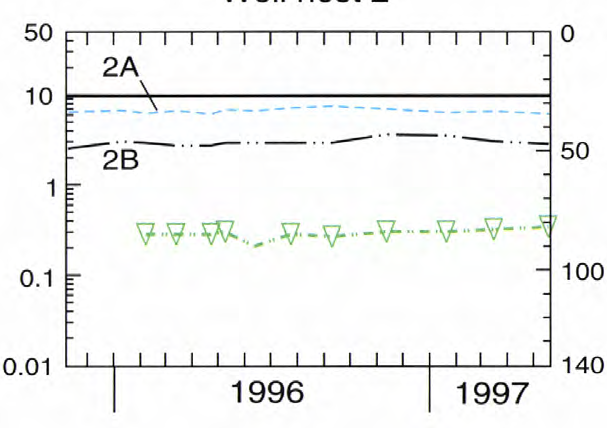

Well nest 5

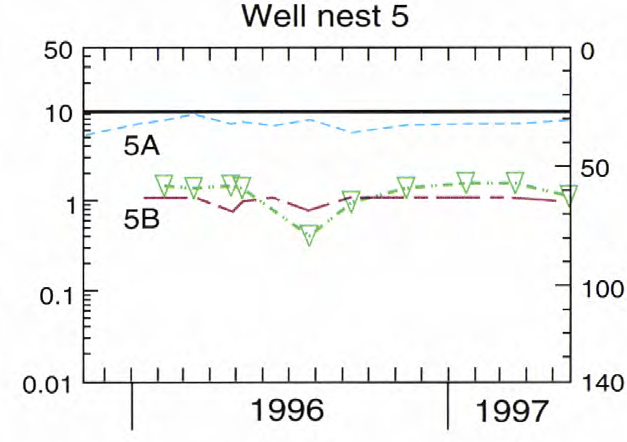

Well nest 8

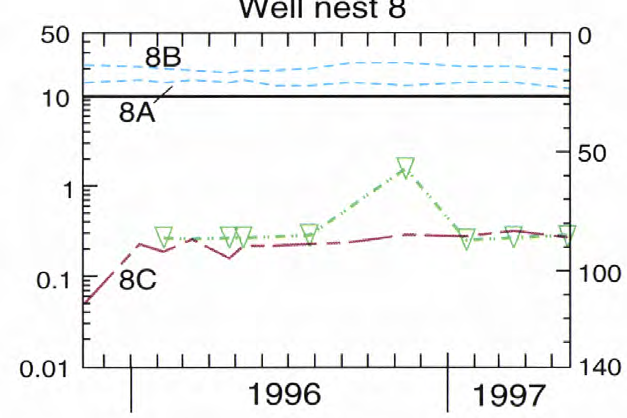

Well nest 3

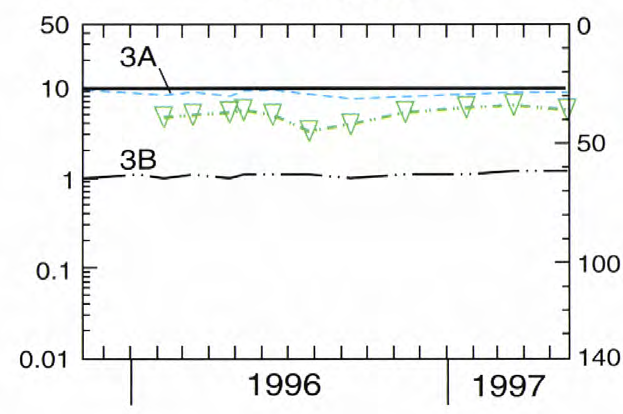

Well nest 6

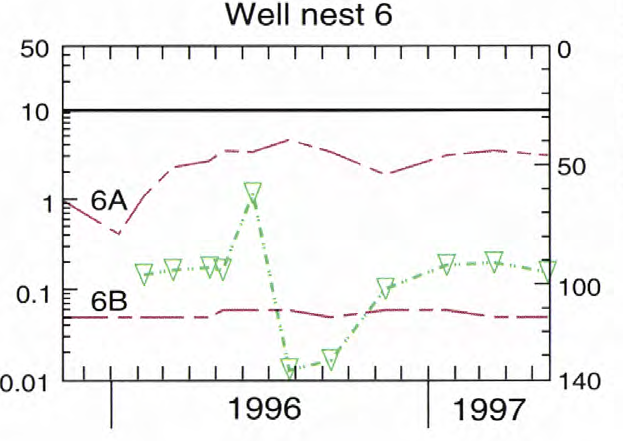

EXPLANATION

Shallow part of the High Plains aquifer, unconfined

__ Deep part of the High Plains aquifer, confined

-..- Deep part of the High Plains aquifer, unconfined

-... - Static water level in shallowest well (right side)

Maximum Contaminant Level

Figure 13. Trends in nitrate concentrations in water samples from nested wells, Upper Big Blue Natural Resources District, central Nebraska, November 1995 through May 1997. 


\section{Spatial Trends in Major-lon and Tritium Concentrations in Ground Water}

In September 1996, ground-water samples were collected from all the nested wells and were analyzed for alkalinity, residue on evaporation at $180^{\circ} \mathrm{C}$, and major ions (table 15 , at back of report) to provide background information on general water chemistry. Tritium analyses were performed on water samples collected from wells $1 \mathrm{~A}, 1 \mathrm{~B}, 6 \mathrm{~A}, 6 \mathrm{~B}$, $8 \mathrm{~A}, 8 \mathrm{~B}$, and $8 \mathrm{C}$ to estimate the age of ground water, and on a precipitation sample collected at well site 8 on June 22, 1997. The precipitation sample had a tritium concentration of $45 \mathrm{pCi} / \mathrm{L}$ (picocuries per liter) tritium.

The results from this study indicate that water in the High Plains aquifer generally is a calciumbicarbonate type, confirming the findings of Johnson and Keech (1959), Keech and Dreeszen (1968a), Ginsberg (1983), Gottula (1990), and Gottula and Link (1992). The relative major-ion composition of the water did not vary much spatially. Generally, the water was hard and locally contained large concentrations of iron (as much as $140 \mu \mathrm{g} / \mathrm{L}$ ) and manganese (as much as $850 \mu \mathrm{g} / \mathrm{L}$ ). Water from well $6 \mathrm{~A}$ contained large concentrations of manga-nese $(670 \mu \mathrm{g} / \mathrm{L})$ and sulfate $(190 \mu \mathrm{g} / \mathrm{L})$, and water from well $6 \mathrm{~B}$ contained large concentrations of iron $(140 \mu \mathrm{g} / \mathrm{L})$ and manganese $(850 \mu \mathrm{g} / \mathrm{L})$ (table 15). Locally, water from the High Plains aquifer may exceed the Secondary Maximum Contaminant Levels for drinking water of $50 \mu \mathrm{g} / \mathrm{L}$ for manganese (U.S. Environmental Protection Agency, 1996).

Previous work suggests that water from the deep part of the High Plains aquifer tends to be a sodium-carbonate type (Keech, 1978). Water from the Dakota aquifer generally is a sodiumbicarbonate type and has lower amounts of alkalinity in Butler County (Ginsberg, 1983), but is a calciumsodium-bicarbonate type in Seward County (Keech, 1978).

Tritium is a radioactive isotope of hydrogen with a half-life of 12.43 years. In 1952, aboveground testing of thermonuclear devices resulted in the release of large amounts of tritium to the atmosphere. Tritium concentrations in water samples from the nested wells varied considerably depending upon the part of the High Plains aquifer in which the wells are completed. Water samples from the shallow part of the High Plains aquifer had the largest tritium concentrations varying from $26 \mathrm{pCi} / \mathrm{L}$ in well $1 \mathrm{~A}$ screened from 90 to $95 \mathrm{ft}$ to $39 \mathrm{pCi} / \mathrm{L}$ in well 8A screened from 90 to $100 \mathrm{ft}$ (table 15). Tritium concentrations in water samples from the deep, confined part of the High Plains aquifer varied from less than $0.3 \mathrm{pCi} / \mathrm{L}$ in well $1 \mathrm{~B}$ screened from 200 to 205 , and well $8 \mathrm{C}$ screened from 225 to $300 \mathrm{ft}$, to $2.5 \mathrm{pCi} / \mathrm{L}$ in well $6 \mathrm{~B}$ screened from 240 to $247 \mathrm{ft}$.

Tritium concentration is related to the age of the water collected. Larger tritium concentrations (about 40-45 pCi/L) would occur in water recharged from about 1953 through the 1970 s, whereas smaller concentrations indicate water older than 1953 (Druliner, 1997). The tritium data indicate that water from the shallow part of the High Plains aquifer, likely recharged during the last 10 to 20 years, is younger, while water from the deep part of the High Plains aquifer, likely recharged more than 50 years ago, is older. The tritium data indicate that, where confined conditions exist in the deep part of the High Plains aquifer and where confining conditions have not been modified through penetration of wells, the water could remain unaffected by nitrate contamination, if water from the shallow part of the High Plains aquifer that is contaminated with nitrate is not reaching the area along longer flow paths from laterally distant areas.

Locally, denitrification also can play a role in reducing the nitrate concentrations. In areas where the lower part of the High Plains aquifer is unconfined, such as at wells $2 \mathrm{~B}, 3 \mathrm{~B}$, and $4 \mathrm{~B}$, the deep part of the High Plains aquifer might be affected by nitrate contamination (fig. 13; table 13). In areas where the aquifer appears confined, some leakage may occur into the deep, confined part of the High Plains aquifer, as suggested by the detectable amounts of tritium in samples collected from wells $6 \mathrm{~A}(1.6 \mathrm{pCi} / \mathrm{L})$ and $6 \mathrm{~B}(2.5 \mathrm{pCi} / \mathrm{L})$ (table 15$)$ or they could be baseline values not necessarily indicating leakage. 


\section{Temporal Trends in Nitrate Concentrations and Field Measurements in Ground Water}

Samples were collected from the nested wells monthly from November 1995 through July 1996, which was modified to bimonthly sample collection from July 1996 through May 1997. The results of this sampling effort show that the nitrate concentrations in water from the nested wells generally did not vary much over this 19 -month period of data collection (fig. 13; table 13). However, there appears to be a small gradual increase in nitrate concentrations in water samples collected from well 1A (fig. 13; table 13). Little seasonal variation in nitrate concentrations was observed. Because of the limited variation in nitrate concentrations and the short period of data collection, trend analyses were not performed.

Nitrate concentrations in water samples from well $1 \mathrm{~A}$ increased from $12 \mathrm{mg} / \mathrm{L}$ on November 8 , 1995, to $21 \mathrm{mg} / \mathrm{L}$ on May 19, 1997 (table 13). Nitrate concentrations in water samples from wells $6 \mathrm{~A}$ and 7A also increased over time, with increases during the first 4 months of sample collection followed by apparent stabilization in nitrate concentrations (fig. 13). Overall, variable nitrate concentrations were noted in water from several wells (table 13).

The water temperature generally was higher during the summer in water samples from wells completed in the unconfined part of the High Plains aquifer than in water samples from wells completed in the confined part of the High Plains aquifer (table 13). Concentrations of dissolved oxygen and $\mathrm{pH}$ did not fluctuate with time. The data suggest that representative sampling of the nested wells generally may be performed during any time of the year and that biannual sampling in the spring and fall may be sufficient to establish trends in water quality.

\section{SPATIAL TRENDS IN NITRATE CON- CENTRATIONS IN SEDIMENT OF THE UNSATURATED ZONE}

Two-hundred and fifty unsaturated-zone sediment samples were collected during three samplings: (1) near the eight nested-well sites (or
14 subsites) (table 6) in November and December 1995; (2) near the same eight nested-well sites (or 14 subsites) in December 1996 and January 1997; and (3) near three nested-well sites (or seven subsites) in March 1997 (figs. 14 through 16, and table 16, at back of report). In November and December 1995, unsaturated-zone samples were collected from $0.25 \mathrm{ft}$ to a depth of as much as $90 \mathrm{ft}$. In December 1996 and January 1997, and in March 1997, unsaturated-zone samples were collected to a depth of as much as $31 \mathrm{ft}$. The nitrate concentrations of the 245 unsaturated-zone samples analyzed varied from less than 0.05 to $69 \mathrm{mg} / \mathrm{kg}$ (milligrams per kilogram) and had a median concentration of $3.7 \mathrm{mg} / \mathrm{kg}$. Fifty percent of the unsaturated-zone samples had nitrate concentrations between 2.15 and $5.6 \mathrm{mg} / \mathrm{kg}$. Five additional samples were collected, but were lost during shipment or processing.

The depth from which the unsaturated-zone samples were collected varied from 0.25 to $90 \mathrm{ft}$ with a median of $10 \mathrm{ft}$. Fifty percent of these samples were collected from a depth varying between 3 and $25 \mathrm{ft}$. Five to 13 samples were collected from each subsite (table 6) depending upon the hydrologic conditions at the site. Statistical analyses of the data indicate that: nitrate concentrations (two-sided p-value $=0.00,245$ samples $)$ varied among sample subsites (Kruskal-Wallis test). The Spearman rank correlation coefficient indicates that the nitrate concentrations of the unsaturated-zone samples were not related to the depth at which the samples were collected.

Overall, the nitrate concentrations of the unsaturated-zone samples at each site remained similar from one sampling to the next. At site DH4, nitrate concentrations in unsaturated-zone samples for 1995, 1996, and 1997 below nonirrigated cropland were larger than those below irrigated cropland or pasture land (figs. 14-16). At site DH7, the differences in nitrate concentrations in unsaturated-zone samples between irrigated and nonirrigated crop land were not as pronounced as at site DH4. The differences at site DH4 in nitrate concentrations may be the result of dilution and rapid transport of nitrate through the unsaturated zone beneath irrigated land compared to the rate of transport beneath nonirrigated land. Other variables, 
such as the amount of nitrogen applied, soil type, organic matter content of the sediments, and the shrink-swell potential of the sediments, especially in the root zone, also could account for differences or nondifferences. For example, at site DH7 nondifferences in nitrate concentrations may be caused by an "overshadowing" of these other factors.

Nitrate concentrations in the unsaturated-zone samples below pasture land generally appear to be smaller than in unsaturated-zone samples below cropland, especially at site DH6, which is similar to the findings of Gottula and Link (1992). This is reasonable because nitrogen fertilizer was not applied to these areas, although excrement from horses is a potential source of nitrogen near subsite $\mathrm{DH} 4 \mathrm{~B}$. At site DH3, the nitrate concentrations in unsaturated-zone samples below the upland (subsite DH3B) were smaller than on the bottomland (subsite DH3A) (figs. 14 and 15). This difference in nitrate concentration could result from transport of nitrogen in surface runoff from the upland to the bottomland.
On the bottomland at site DH3A, a larger amount of nitrate will tend to accumulate than on sloping upland or foot slopes, probably resulting in more nitrate being available to leach into the unsaturated zone.

At subsite DH7A, an increase in nitrate concentrations with depth in the unsaturated zone was noted from 1995 to 1996 (figs. 14 and 15). This apparent increase in unsaturated-zone nitrate concentrations with depth could have resulted from differences in nitrogen application. Nitrogen fertilizer was applied in 1995, but was not applied to cropland in 1996 (table 6). At site DH8 no obvious differences in nitrate concentrations of unsaturatedzone samples were noted. The unsaturated-zone profile is intended to be monitored about every 3 years in the future for nitrate or nitrogen species concentrations by the UBBNRD as BMPs are implemented in the study area (R.D. DeBuhr, Upper Big Blue Natural Resources District, oral commun., 1998). 
$\mathrm{DH} 1$

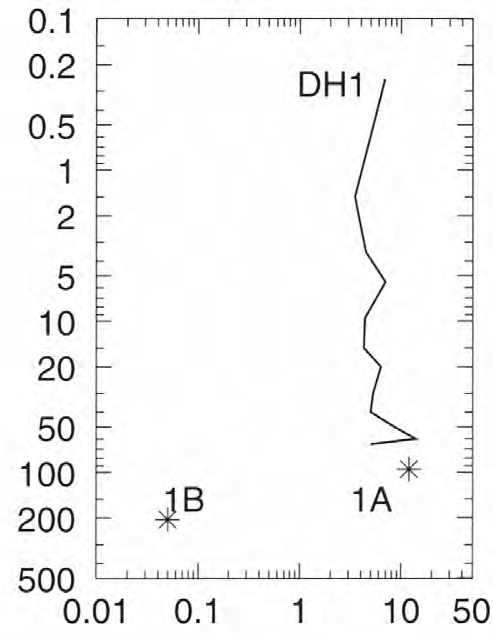

$\mathrm{DH} 4$

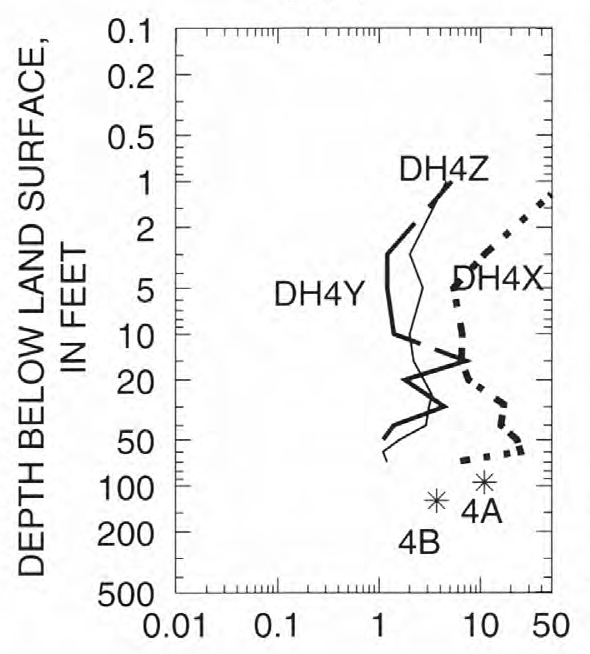

$\mathrm{DH} 7$

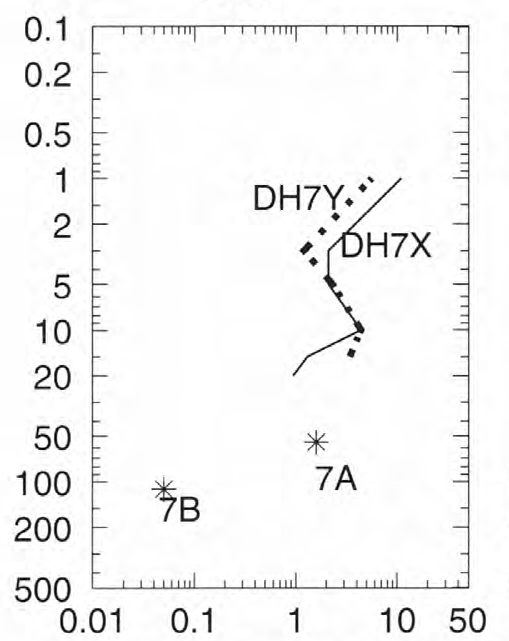

$\mathrm{DH} 2$

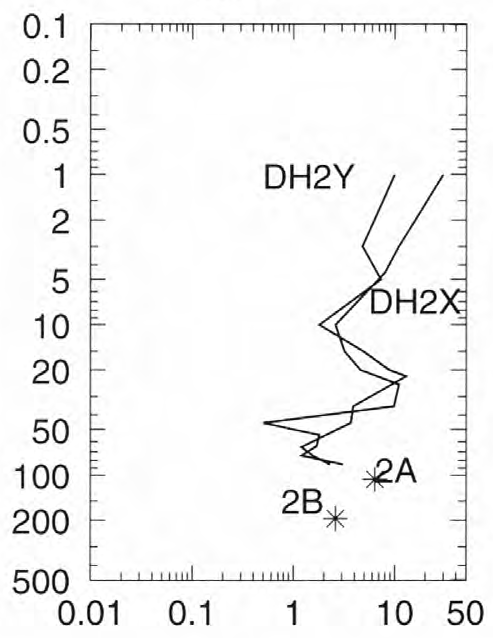

$\mathrm{DH} 5$

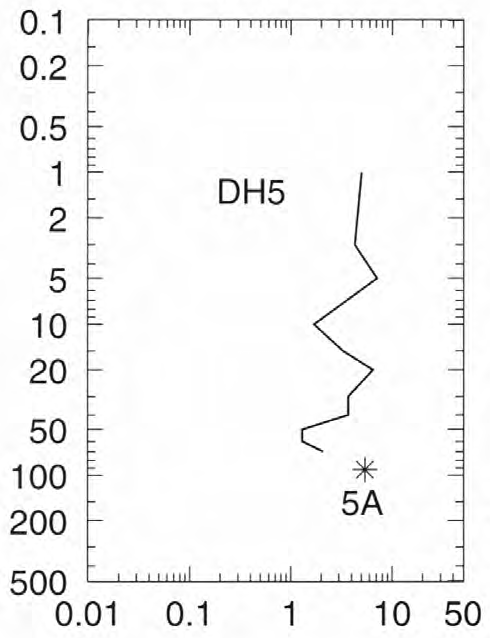

$\mathrm{DH} 8$

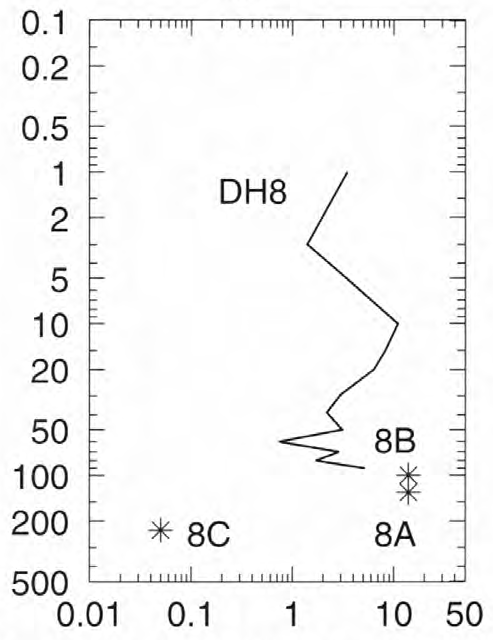

$\mathrm{DH} 3$

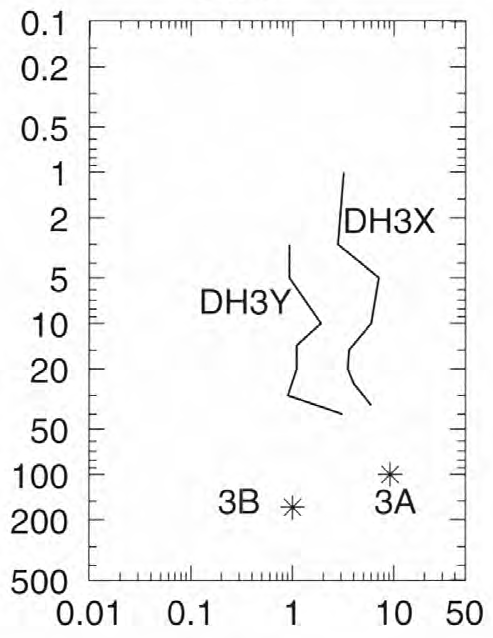

$\mathrm{DH} 6$

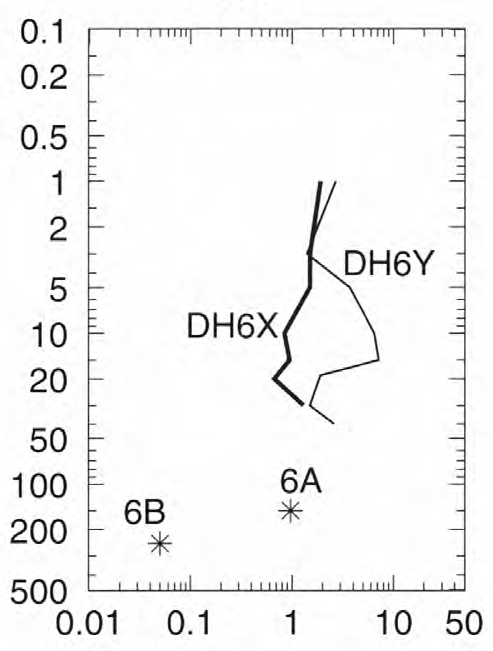

EXPLANATION

DH8 Total nitrate concentration below irrigated cropland and local subsite identification number

DH6X

Total nitrate concentration below pasture land use and local subsite identification number

$\mathrm{DH} 7 \mathrm{Y}$

..... Total nitrate concentration below nonirrigated cropland and local subsite identification number

* $\quad$ Aissolved nitrate concentration in water from nearby well and local well identification number.

Figure 14. Nitrate concentrations in unsaturated-zone sediment samples from November and December 1995 and in water samples from nested wells from November 1995, by depth, Upper Big Blue Natural Resources District, central Nebraska. 
$\mathrm{DH} 1$

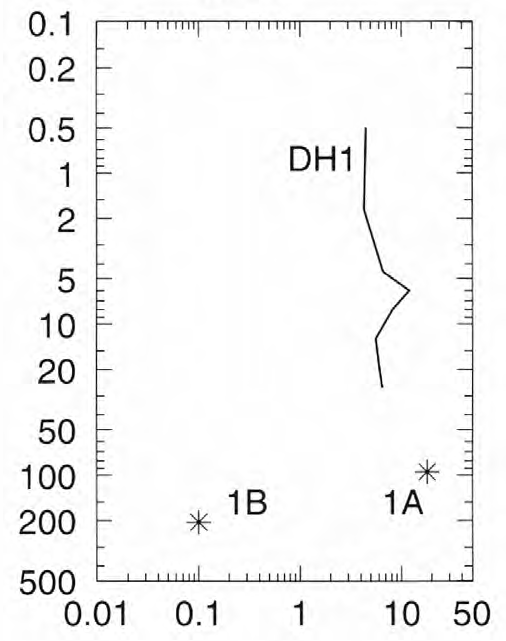

$\mathrm{DH} 4$

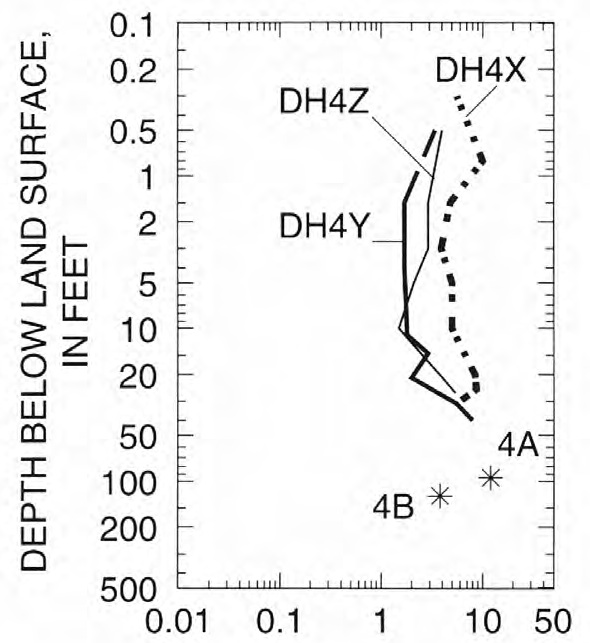

$\mathrm{DH} 7$

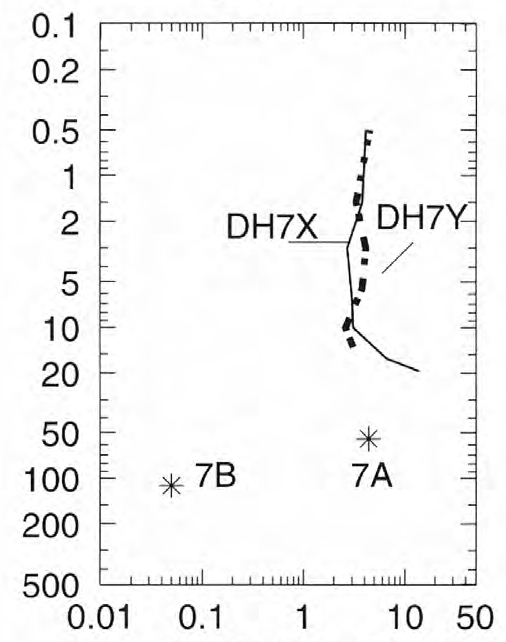

$\mathrm{DH} 3$
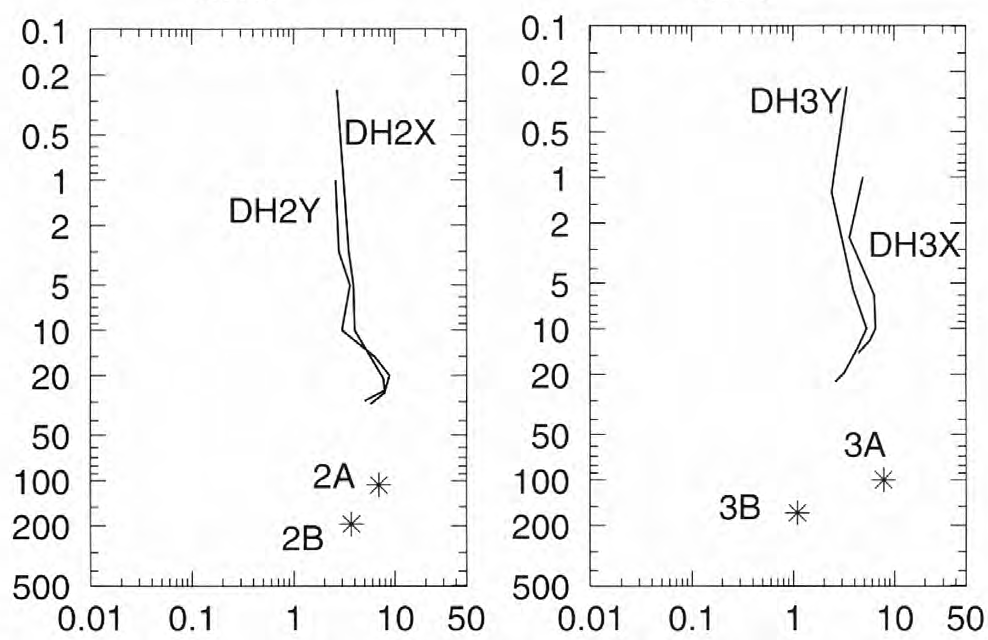

DH 5

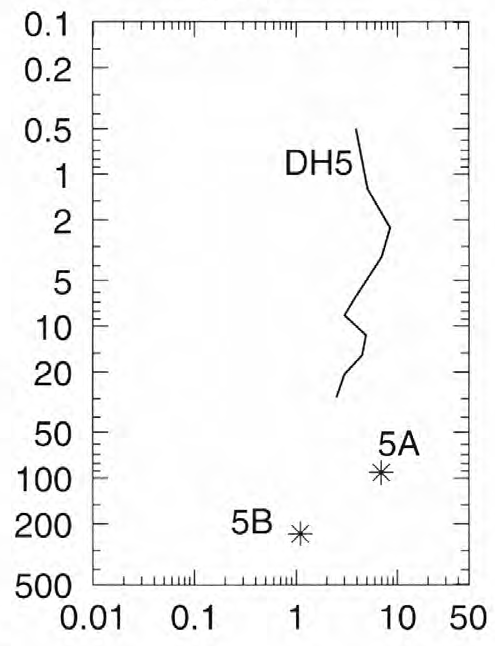

$\mathrm{DH} 6$

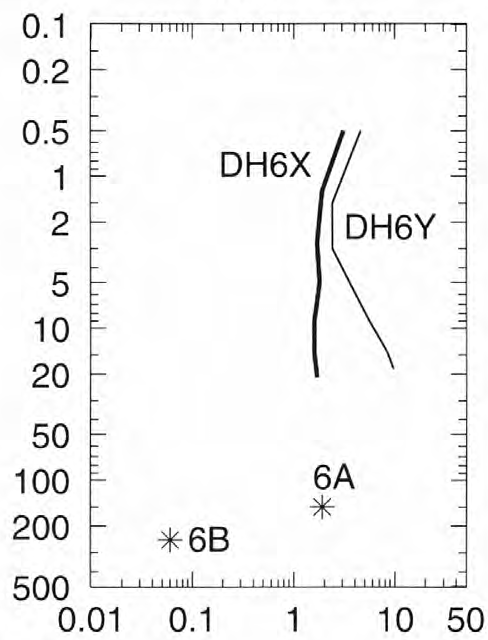

\section{EXPLANATION}

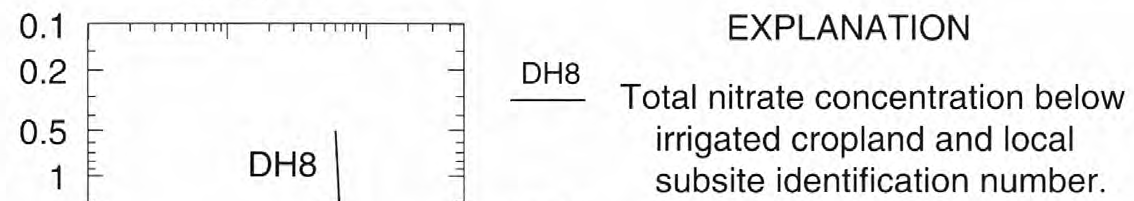

DH6X

Total nitrate concentration below pasture land use and local subsite identification number.

DH7Y ... Total nitrate concentration below nonirrigated cropland and local subsite identification number.

$8 \mathrm{~A}$

Dissolved nitrate concentration in water from nearby well and local well identification number.

Figure 15. Nitrate concentrations in unsaturated-zone sediment samples from December 1996 and January 1997, and in water samples from nested wells from November 1996, by depth, Upper Big Blue Natural Resources District, central Nebraska. 
$\mathrm{DH} 4$

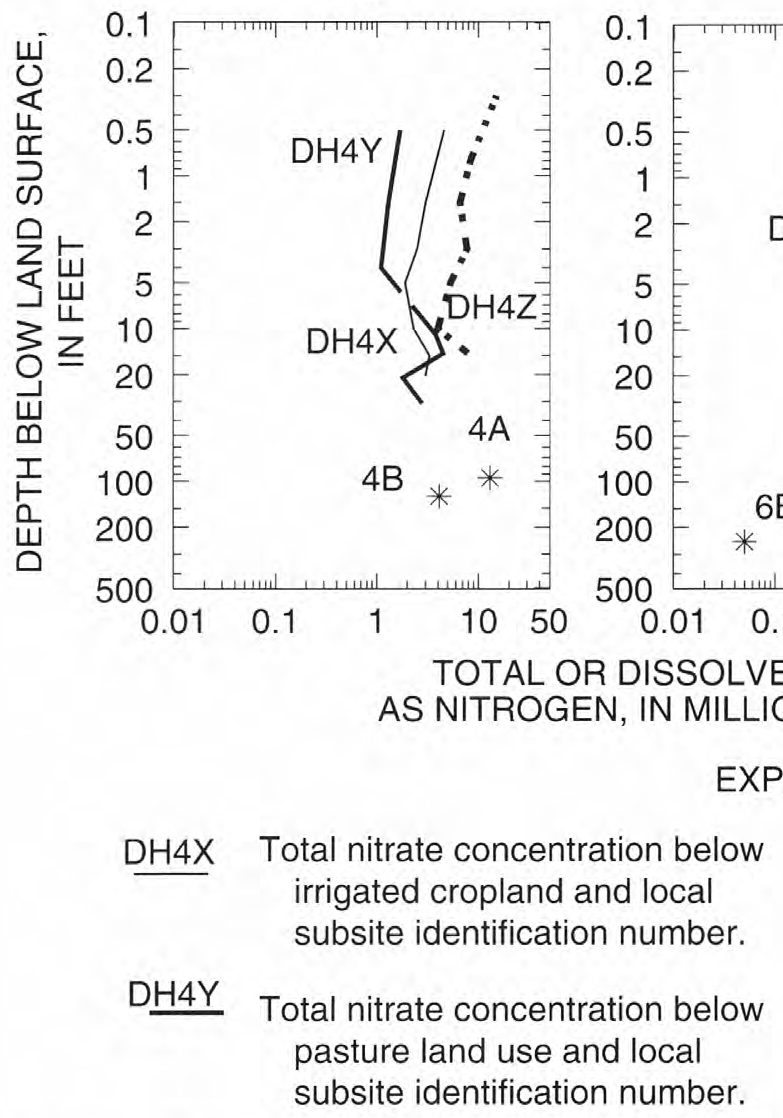

$\mathrm{DH} 6$

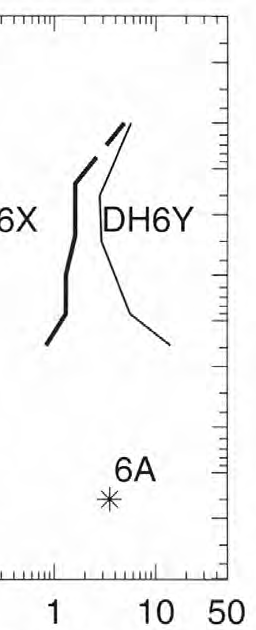

$\mathrm{DH} 7$

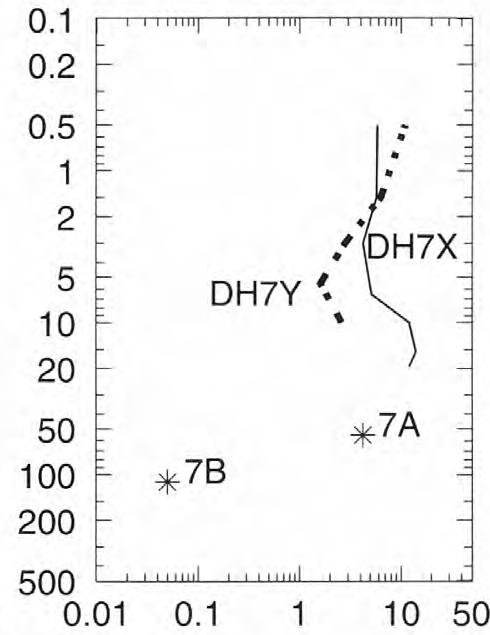

NITRATE CONCENTRATION
MS PER KILOGRAM OR LITER

EXPLANATION

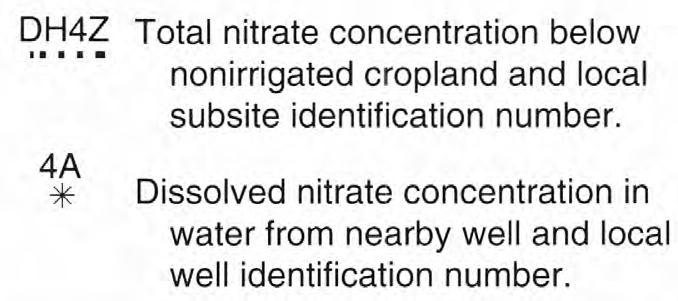

Figure 16. Nitrate concentrations in unsaturated-zone sediment samples from March 1997, and in water samples from nested wells from March 1997, by depth, Upper Big Blue Natural Resources District, central Nebraska.

\section{SUMMARY AND CONCLUSIONS}

Following the designation of the UBBNRD as a Special Protection Area by the Nebraska Department of Environmental Quality in 1993, the UBBNRD requested that the U.S. Geological Survey develop a monitoring approach to identify the spatial and temporal variability of nitrate concentrations in ground water and assess the presence of nitrate concentrations in the unsaturated zone. A groundwater-quality monitoring network of 197 domestic and registered wells, along with 8 nested-well sites (a total of 18 wells) and associated unsaturated-zone subsites was proposed during this study. Of the 197 wells in the monitoring-well network, 104 wells are completed in the shallow part of the High Plains aquifer, 86 are completed in the deep part of the High Plains aquifer, 3 are completed in both parts of this aquifer, 1 is completed in the Greenhorn Formation (well 1001), and 3 are completed in the Dakota aquifer. Of the 193 wells completed in the High Plains aquifer, 123 are screened in an unconfined part of the High Plains aquifer and 70 are screened in the confined part of the High Plains aquifer. Eight nested-well sites were selected for the installation of 18 nested wells, based on the hydrogeologic conditions, the management zones, the presence of irrigated cropland, and other selected reasons. Unsaturated-zone samples were collected near each nested-well site. Unsaturated-zone subsites were selected based on land use or landscape position.

Nitrate concentrations in the ground water in the UBBNRD ranged from 0.06 to $27 \mathrm{mg} / \mathrm{L}$ and had a median concentration of $4.6 \mathrm{mg} / \mathrm{L}$. Of the 197 samples collected from the ground-waterquality monitoring network, 41 samples (21 percent) exceeded the U.S. Environmental Protection Agency Maximum Contaminant Level for drinking water of 
$10 \mathrm{mg} / \mathrm{L}$ for nitrate as nitrogen. In the shallow part of the High Plains aquifer, nitrate concentrations ranged from 0.09 to $27 \mathrm{mg} / \mathrm{L}$ and had a median concentration of $6.3 \mathrm{mg} / \mathrm{L}$. In the deep part of the High Plains aquifer, the nitrate concentrations ranged from 0.06 to $21 \mathrm{mg} / \mathrm{L}$ and had a median concentration $3.4 \mathrm{mg} / \mathrm{L}$. In the Dakota aquifer, the median nitrate concentration was $0.11 \mathrm{mg} / \mathrm{L}$. The median nitrate concentrations in the unconfined and the confined part of the High Plains aquifer were 6.2 and $2.9 \mathrm{mg} / \mathrm{L}$, respectively.

Among the management zones, the median nitrate concentrations varied from 0.97 to $10 \mathrm{mg} / \mathrm{L}$. The median nitrate concentrations (in $\mathrm{mg} / \mathrm{L}$ ) by zone ranked from small to large were: 0.97 in zone $12,1.6$ in zone 10, 2.6 in zone 8,3.3 in zone 3, 3.6 in zone 9 , 4.7 in zones 1 and 7, 4.9 in zone 4, 5.0 in zone 2, 6.2 in zone $6,6.7$ in zone 11 , and 10 in zone 5 . The median nitrate concentration $(10 \mathrm{mg} / \mathrm{L})$ of water samples from wells in zone 5 exceeded the Phase II trigger of $9 \mathrm{mg} / \mathrm{L}$.

Nitrate concentrations in water from the Dakota aquifer and water from the deep part of the High Plains aquifer were significantly smaller than concentrations in water from the shallow part of the High Plains aquifer. Nitrate concentrations and dissolved oxygen differed significantly depending upon the hydrogeologic condition of the aquifer from which the water was derived. Nitrate concentrations and dissolved oxygen tended to be larger in the unconfined part of the High Plains aquifer, indicating no denitrifying conditions existed. Smaller concentrations of nitrate were detected in ground-water samples from wells in zones 8 and 12, than in water samples from the other zones. Larger nitrate concentrations were detected in water samples from wells in zones 11,6, and 5, than in the remaining zones.

Two hundred thirty-two water samples were collected from the eight nested-well sites from November 1995 to May 1997. The nitrate concentrations and field measurements of water samples collected from the nested wells generally agreed with results from the proposed ground-waterquality monitoring network of domestic and registered wells. They show the largest nitrate concentrations in samples from the unconfined part of the High Plains aquifer, intermediate nitrate concentrations in samples from the unconfined, deep part of the High Plains aquifer, and the smallest nitrate concentrations in samples from the confined part of the High Plains aquifer. Water samples collected from the various parts of the High Plains aquifer had the following median concentrations: shallow, $9.2 \mathrm{mg} / \mathrm{L}$; deep, $0.42 \mathrm{mg} / \mathrm{L}$; unconfined, $7.1 \mathrm{mg} / \mathrm{L}$; and confined, $0.10 \mathrm{mg} / \mathrm{L}$.

Statistical analyses showed that nitrate concentrations varied significantly by site, aquifer, and hydrogeologic condition. In addition, statistical analyses of data collected from the nested wells supported the relations of the nitrate concentrations and field measurements determined with the data from the proposed ground-water-quality monitoring network. The data suggest that the shallow part of the High Plains aquifer appears to be very susceptible to contamination with nitrate. Nitrate contamination of the High Plains aquifer is of most concern where (1) the shallow part of the High Plains aquifer is thin and in areas where confining conditions are absent, or (2) where cross-contamination of unconfined and confined parts of the High Plains aquifer occurs. The data also suggest that representative sampling of the nested wells may be performed during any time of the year, and that biannual sampling in the spring and fall may be sufficient to establish trends in water quality based on the best management practices adopted by the UBBNRD.

In September 1996, water samples were collected from all the nested wells and analyzed for alkalinity, residue on evaporation at $180^{\circ} \mathrm{C}$, and major ion and tritium concentrations. The results suggest that water in the High Plains aquifer in the study area generally is a calcium-bicarbonate type and does not vary much spatially. Locally, the High Plains aquifer may exceed the USEPA Secondary Maximum Contaminant Standard of $50 \mu \mathrm{g} / \mathrm{L}$ for manganese.

Water samples from the shallow part of the High Plains aquifer had the largest tritium content, which varied from $26 \mathrm{pCi} / \mathrm{L}$ to $39 \mathrm{pCi} / \mathrm{L}$. Tritium activity in the deep confined part of the High Plains aquifer varied from less than $0.3 \mathrm{pCi} / \mathrm{L}$ to $2.5 \mathrm{pCi} / \mathrm{L}$. The tritium data, therefore, indicate that water samples from the shallow part of the High Plains aquifer, likely recharged during the last 10 to 20 years, is younger, while water samples from the 
deep part of the High Plains aquifer, likely recharged more than 50 years ago, is older. The tritium data also indicate that, where confined conditions exist in the deep part of High Plains aquifer and have not been modified through penetration of wells, the water could remain unaffected by nitrate contamination. However, generally, the water under confined conditions has measurable amounts of nitrate.

Unsaturated-zone sediment samples were collected three times at 14 subsites and were analyzed for nitrate. The nitrate concentrations detected in 245 of 250 samples collected varied from less than 0.05 to $69 \mathrm{mg} / \mathrm{kg}$ and had a median of $3.7 \mathrm{mg} / \mathrm{kg}$. The depth from which these samples were collected varied from 0.25 to $90 \mathrm{ft}$ with a median of $10 \mathrm{ft}$. Nitrate concentrations varied among sample subsites and were not related to the depth at which the samples were collected. At site DH4, nitrate concentrations in unsaturated-zone samples below nonirrigated cropland were larger than concentrations from samples below irrigated cropland and pasture land. Nitrate concentrations in unsaturated-zone sediment samples below pasture land generally were smaller than nitrate concentrations in samples from below cropland.

Thus, water from the High Plains aquifer, especially in the shallow unconfined part, is more affected by nonpoint-source nitrogen than the water present in the Dakota aquifer. However, the confined part of the High Plains aquifer also has been contaminated with nitrogen probably in part because of (1) some leakage through the confining layer, (2) long flow paths from unconfined to confined parts of the aquifer, or (3) cross-contamination caused by well construction and well completions. In summary, the potential for large nitrate concentrations in the High Plains aquifer is of most concern where (1) the shallow part of the High Plains aquifer is thin and in areas where confining conditions are absent, such as in parts of Seward and York Counties, or (2) where cross-contamination of unconfined and confined parts of the High Plains aquifer occurs, such as in York County.

For management purposes, the management areas of concern are management zones 5, 6, and 11 . To identify trends in nitrate concentrations that may be associated with the implementation of best management practices in the study area, the UBBNRD could: (1) collect and analyze water samples from the proposed ground-watermonitoring network of domestic and registered irrigation wells every 1 to 3 years; (2) collect and analyze water samples from the nested wells in spring and fall; and (3) collect and analyze soil samples from the unsaturated-zone profile about every 3 years.

\section{REFERENCES}

Burchett R.R. and Smith, F.A., 1993, Nebraska Water Survey Test-Hole Report 80, Seward County: Conservation and Survey Division, Institute of Agriculture and Natural Resources, University of Nebraska-Lincoln, 48 p.

1994, Nebraska Water Survey Test-Hole Report 18, Clay County: Conservation and Survey Division, Institute of Agriculture and Natural Resources, University of NebraskaLincoln, 35 p.

1996, Nebraska Water Survey Test-Hole Report 72, Polk County: Conservation and Survey Division, Institute of Agriculture and Natural Resources, University of NebraskaLincoln, 44 p.

Conservation and Survey Division and U.S. Geological Survey, 1953, Logs of test holes, Fillmore County, Nebraska: Conservation and Survey Division, University of Nebraska and U.S. Geological Survey, 22 p.

Dugan, J.T., and Zelt, R.B., in press, Simulation and analysis of soil-water conditions in the Great Plains and adjacent areas, central United States, 1951-80: U.S. Geological Survey Water-Supply Paper 2427.

Druliner, A.D., 1997, Water quality and chemical evolution of ground water in the Long Pine Creek Area, Brown and Rock Counties, Nebraska, 1993-94: U.S. Geological Survey Water-Resources Investigations Report 96-4095, 22 p.

Ellis, M.J., 1986, Hydrogeologic data for the Dakota aquifer system in Nebraska: U.S. Geological Survey Open-File Report 86-526, 100 p.

Emery, P.A., 1966, Geohydrology of Saline County, Nebraska: U.S. Geological Survey Hydrologic Investigations Atlas HA-216, 1 sheet. 
Fishman, J.J., and Friedman, L.C., eds., 1989, Methods for determination of inorganic substances in water and fluvial sediments: U.S. Geological Survey Techniques of WaterResources Investigations, book 5, chap. A1, 545 p.

Ginsberg, M.H., 1983, Hydrogeology of Butler County, Nebraska: Conservation and Survey Division, Institute of Agriculture and Natural Resources, University of Nebraska-Lincoln, Nebraska, Water Survey Paper 55, 78 p.

Gottula, J.J., 1990, A study of nonpoint-source ground-water contamination in the eastern portion of the Upper Big Blue Natural Resources District-A Special Protection Area report: Nebraska Department of Environmental Quality, Water Quality Division, Ground Water Section, $57 \mathrm{p}$.

Gottula, J.J., and Link, M.L., 1992, A study of nonpoint-source ground-water contamination in the western portion of the Upper Big Blue Natural Resources District-A Special Protection Area report: Nebraska Department of Environmental Quality, Water Quality Division, Ground Water Section, 48 p.

Helsel, D.R., and Hirsch, R.M., 1992, Statistical methods in water resources: New York, Elsevier Publishers, $522 \mathrm{p}$.

Johnson, C.R., and Keech, C.F., 1959, Geology and ground-water resources of the Big Blue River Basin above Crete, Nebraska, with a section on Chemical quality of water, by Robert Brennan: U.S. Geological Survey Water-Supply Paper 1474, $94 \mathrm{p}$.

Jones, B.E., 1987, Quality control manual of the U.S. Geological Survey's National WaterQuality Laboratory: U.S. Geological Survey Open-File Report 87-457, 17 p.

Keech, C.F., 1960, Nebraska Water Survey Test Hole Report 3, Hamilton County: Conservation and Survey Division, University of Nebraska, and Ground Water Branch, U.S. Geological Survey, $53 \mathrm{p}$.

1962, Ground-water resources of Hamilton County, Nebraska, with a section on Chemical quality of water, by P.G. Rosene:

U.S. Geological Survey Water-Supply Paper $1539-\mathrm{N}, 64 \mathrm{p}$.

1978, Water resources of Seward County, Nebraska, with section on The quality of water, by R.A. Engberg: Conservation and Survey Division, Institute of Agriculture and Natural
Resources, University of Nebraska-Lincoln, Nebraska Water Survey Paper 46, 88 p.

Keech, C.F., and Dreeszen, V.H., 1959, Geology and ground-water resources of Clay County, Nebraska, with a section on Chemical quality of water, by F.H. Rainwater: U.S. Geological Survey Water-Supply Paper 1468, 157 p.

1968a, Geology and ground-water resources of Fillmore County, Nebraska, with a section on Chemical quality of water, by L.R. Petri: U.S. Geological Survey Water-Supply Paper 1839-L, 27 p.

1968b, Availability of ground water in Adams County, Nebraska: U.S. Geological Survey Hydrologic Investigations Atlas HA-287.

Keech, C.F., and Dreeszen, V.H., and Emery, P.A., 1967, Availability of ground-water in York County, Nebraska: U.S. Geological Survey Water-Supply Paper 1839-F, 17 p.

Kolpin, D.W., Burkart, M.R., and Thurman, E.M., 1994, Herbicide and nitrate in near surface aquifers in the Midcontinental United States, 1991: U.S. Geological Survey Water-Supply Paper 2413, $34 \mathrm{p}$.

Lugn, A.L., and Wenzel, L.K., 1938, Geology and ground-water resources of south-central Nebraska, with special reference to the Platte River valley between Chapman and Gothenburg: U.S. Geological Survey WaterSupply Paper 779, 242 p.

Page, A.L., Miller, R.H., and Keeney, D.R., 1982, Methods of soil analyses, Part 2-Chemical and microbiological properties: American Society of Agronomy, Inc., p. 679-682.

Pettijohn, R.A., and Chen, H.H., 1983a, Geohydrology of the High Plains aquifer system in Nebraska: U.S. Geological Survey WaterResources Investigations Open-File Report 82-502, 3 pls.

1983b, Hydraulic conductivity, specific yield, and pumpage-High Plains aquifer system, Nebraska: U.S. Geological Survey WaterResources Investigations Report 82-4014, 3 pls.

Pritt, J.W., and Jones, B.E., eds., 1989, 1990 National Water Quality Services Catalog: U.S. Geological Survey Open-File Report 89-386, 132 p.

Smith, F.A., 1963, Nebraska Water Survey Test Hole Report 5, York County: Conservation and Survey Division, University of Nebraska, and 
Ground Water Branch, U.S. Geological Survey, $60 \mathrm{p}$.

Smith, F.A., Emery, P.A., and Souders, V.L., 1964,

Nebraska Water Survey Test Hole Report 6,

Saline County: Conservation and Survey

Division, University of Nebraska, and

U.S. Geological Survey, 82 p.

Verstraeten, I.M., and Ellis, M.J., 1995,

Reconnaissance of ground-water quality in the Papio-Missouri River Natural Resources

District, eastern Nebraska, July through

September 1992: U.S. Geological Survey

Water-Resources Investigations

Report 94-4197, 90 p.

Upper Big Blue Natural Resources District, 1994, Special Protection Area Action Plan, York, Nebraska, May 19, 1994: Upper Big Blue

Natural Resources District, 29 p.

U.S. Environmental Protection Agency, 1996, Drinking water regulations and health advisories: U. S. Environmental Protection Agency, Office of Water, $11 \mathrm{p}$. 


\section{Supplemental Data-Water-Quality Results (Tables 8, 9, 13, 14, 15, and 16)}


Table 8. Nitrate concentrations and field measurements for water samples from the ground-water-quality monitoring network of domestic and registered wells, Upper Big Blue Natural Resources District, central Nebraska, July 1995 through September 1997

$[*$, flagged analytical results, because of large variability in nitrate concentrations from year to year; $\mathrm{mg} / \mathrm{L}$, milligrams per liter; $\mu \mathrm{S} / \mathrm{cm}$, microsiemens per centimeter at $25^{\circ} \mathrm{C}$; ${ }^{\circ} \mathrm{C}$, degrees Celsius; UF, unconfined part of the High Plains aquifer; $\mathrm{CF}$, confined part of the High Plains aquifer; sample date, yyyymmdd; --, no data; <, less than]

\begin{tabular}{|c|c|c|c|c|c|c|c|c|c|}
\hline $\begin{array}{c}\text { Well } \\
\text { identification } \\
\text { number }{ }^{1} \\
\text { (fig. } 8)\end{array}$ & $\begin{array}{l}\text { Hydro- } \\
\text { geologic } \\
\text { condition }\end{array}$ & Sample type & $\begin{array}{l}\text { Sample } \\
\text { date }\end{array}$ & $\begin{array}{c}\text { Sample } \\
\text { time }\end{array}$ & $\begin{array}{l}\text { Nitrate and } \\
\text { nitrite as } \\
\text { nitrogen, } \\
\text { dissolved } \\
(\mathrm{mg} / \mathrm{L})\end{array}$ & $\begin{array}{l}\text { Oxygen, } \\
\text { dissolved, } \\
\text { field } \\
\text { (mg/L) }\end{array}$ & $\begin{array}{l}\mathrm{pH}, \\
\text { field } \\
\text { (standard } \\
\text { units) }\end{array}$ & $\begin{array}{c}\text { Specific } \\
\text { conduct- } \\
\text { ance, field } \\
(\mu \mathrm{S} / \mathrm{cm})\end{array}$ & $\begin{array}{c}\text { Water } \\
\text { temperature } \\
\left({ }^{\circ} \mathrm{C}\right)\end{array}$ \\
\hline 0101 & UF & Environmental & 19950829 & 1200 & 5.2 & 6.5 & 7.1 & 580 & 14.5 \\
\hline 0101 & UF & Environmental & 19960716 & 1600 & 4.7 & 3.6 & 7.1 & 614 & 13.5 \\
\hline 0101 & UF & Blank & 19960716 & 1605 & .13 & -- & - & -- & -- \\
\hline 0101 & UF & Duplicate & 19960716 & 1610 & 4.6 & 3.6 & 7.1 & 614 & 13.5 \\
\hline 0102 & UF & Environmental & 19960715 & 1400 & 4.4 & 6.1 & 7.3 & 634 & 13.5 \\
\hline 0103 & UF & Environmental & 19960730 & 0830 & 4.6 & 7.9 & 7.0 & 620 & 12 \\
\hline 0104 & UF & Environmental & 19950829 & 1030 & 5.5 & 6.7 & 7.1 & 660 & 14 \\
\hline 0104 & UF & Environmental & 19960715 & 1553 & 6.6 & 7.1 & 7.2 & 701 & 15 \\
\hline 0105 & UF & Environmental & 19950721 & 1010 & $* 17$ & 5.9 & 7.0 & 1,340 & 13.5 \\
\hline 0105 & UF & Environmental & 19960829 & 1400 & $* 21$ & 9.6 & 6.8 & 1,200 & 12.5 \\
\hline 0106 & UF & Environmental & 19950721 & 1050 & .15 & .5 & 7.5 & 574 & 13 \\
\hline 0106 & UF & Environmental & 19960708 & 1405 & .12 & .1 & 7.1 & 568 & 13 \\
\hline 0107 & UF & Environmental & 19950724 & 1150 & $* 11$ & 8.6 & 7.5 & 752 & 13.5 \\
\hline 0107 & UF & Environmental & 19960710 & 1312 & 9.3 & 1.6 & 7.3 & 769 & 13 \\
\hline 0108 & UF & Environmental & 19950726 & 1000 & .14 & 4.0 & 6.5 & 604 & 13 \\
\hline 0108 & UF & Environmental & 19960710 & 1234 & 3.0 & 3.9 & 7.1 & 622 & 12.5 \\
\hline 0108 & UF & Blank & 19960710 & 1240 & .06 & -- & -. & -. & -- \\
\hline 0108 & UF & Duplicate & 19960710 & 1245 & 3.0 & 3.9 & 7.1 & 622 & 12.5 \\
\hline 0109 & UF & Environmental & 19950727 & 1355 & 1.1 & 1.0 & 7.1 & 679 & 13 \\
\hline 0109 & UF & Environmental & 19960710 & 1420 & 2.2 & 4.5 & 7.2 & 703 & 13 \\
\hline 0110 & UF & Environmental & 19950801 & 1400 & 1.5 & 1.4 & 7.5 & 571 & 13 \\
\hline 0110 & UF & Duplicate & 19950801 & 1405 & 1.3 & 1.4 & 7.5 & 571 & 13 \\
\hline 0110 & UF & Blank & 19950801 & 1410 & $<.05$ & - & - & - & - \\
\hline 0110 & UF & Environmental & 19960719 & 0920 & 1.0 & 1.5 & 7.1 & 587 & 13.5 \\
\hline 0111 & UF & Environmental & 19960719 & 0837 & 6.4 & 5.6 & 7.2 & 628 & 13.5 \\
\hline 0112 & UF & Environmental & 19950721 & 1350 & $* 12$ & 8.0 & 7.1 & 860 & 14.5 \\
\hline 0112 & UF & Environmental & 19960708 & 1240 & $* 13$ & 8.6 & 6.7 & 871 & 14.5 \\
\hline 0113 & UF & Blank & 19950721 & 1305 & $<.05$ & -- & -- & -- & -- \\
\hline 0113 & UF & Environmental & 19950721 & 1306 & $* 11$ & 5.1 & 7.1 & 742 & 13 \\
\hline 0113 & UF & Environmental & 19960708 & 1320 & $* 13$ & 6.5 & 6.9 & 773 & 13 \\
\hline 0114 & UF & Environmental & 19960711 & 956 & $* 21$ & 8.3 & 6.9 & 1,680 & 12 \\
\hline 0115 & UF & Environmental & 19960715 & 1245 & $* 19$ & 7.6 & 7.2 & 1,020 & 12 \\
\hline 0116 & UF & Environmental & 19950721 & 1550 & 2.4 & 7.7 & 6.8 & 443 & 13.5 \\
\hline 0116 & UF & Environmental & 19960710 & 1459 & 1.5 & 8.0 & 6.8 & 352 & 14 \\
\hline 0201 & UF & Environmental & 19950721 & 0905 & 4.0 & 4.2 & 6.9 & 636 & 12.5 \\
\hline 0201 & UF & Environmental & 19960717 & 1515 & 4.5 & 4.1 & 6.6 & 642 & 12.5 \\
\hline 0202 & UF & Environmental & 19950728 & 1115 & $* 11$ & 7.7 & 7.5 & 714 & 13.5 \\
\hline 0202 & UF & Environmental & 19960718 & 1040 & $* 11$ & 6.3 & 6.7 & 692 & 12.5 \\
\hline 0202 & UF & Duplicate & 19960718 & 1045 & $* 11$ & 6.3 & 6.7 & 692 & 12.5 \\
\hline 0202 & UF & Blank & 19960718 & 1050 & .07 & - & -- & -- & -- \\
\hline 0203 & UF & Environmental & 19950719 & 1640 & 9.2 & 7.6 & 7.2 & 718 & 12.5 \\
\hline 0203 & UF & Duplicate & 19950719 & 1645 & 9.3 & 7.6 & 7.2 & 718 & 12.5 \\
\hline
\end{tabular}


Table 8. Nitrate concentrations and field measurements for water samples from the ground-water-quality monitoring network of domestic and registered wells, Upper Big Blue Natural Resources District, central Nebraska, July 1995 through September 1997--Continued

\begin{tabular}{|c|c|c|c|c|c|c|c|c|c|}
\hline $\begin{array}{c}\text { Well } \\
\text { identification } \\
\text { number } \\
\text { (fig. } 8 \text { ) }\end{array}$ & $\begin{array}{l}\text { Hydro- } \\
\text { geologic } \\
\text { condition }\end{array}$ & Sample type & $\begin{array}{l}\text { Sample } \\
\text { date }\end{array}$ & $\begin{array}{l}\text { Sample } \\
\text { time }\end{array}$ & $\begin{array}{l}\text { Nitrate and } \\
\text { nitrite as } \\
\text { nitrogen, } \\
\text { dissolved } \\
(\mathrm{mg} / \mathrm{L})\end{array}$ & $\begin{array}{c}\text { Oxygen, } \\
\text { dissolved, } \\
\text { field } \\
(\mathrm{mg} / \mathrm{L})\end{array}$ & $\begin{array}{c}\mathrm{pH}, \\
\text { field } \\
\text { (standard } \\
\text { units) }\end{array}$ & $\begin{array}{c}\text { Specific } \\
\text { conduct- } \\
\text { ance, field } \\
(\mu \mathrm{S} / \mathrm{cm})\end{array}$ & $\begin{array}{c}\text { Water } \\
\text { temperature } \\
\left({ }^{\circ} \mathrm{C}\right)\end{array}$ \\
\hline 0203 & UF & Blank & 19950719 & 1650 & $<.05$ & -- & - & - & - \\
\hline 0203 & UF & Environmental & 19960718 & 1440 & $* 12$ & 6.1 & 6.7 & 809 & 13 \\
\hline 0204 & UF & Environmental & 19950731 & 1115 & 4.0 & 6.3 & 7.0 & 515 & 12.5 \\
\hline 0204 & UF & Environmental & 19960716 & 1320 & 4.4 & 5.4 & 7.0 & 513 & 12.5 \\
\hline 0205 & $\mathrm{CF}$ & Environmental & 19950727 & 1120 & 1.0 & .5 & 7.4 & 554 & 14 \\
\hline 0205 & $\mathrm{CF}$ & Environmental & 19960718 & 0815 & .82 & .1 & 6.9 & 553 & 13.5 \\
\hline 0206 & UF & Environmental & 19960718 & 1140 & 5.0 & 6.4 & 6.8 & 642 & 13.5 \\
\hline 0207 & UF & Environmental & 19950720 & 1410 & .26 & .5 & 7.1 & 497 & 13 \\
\hline 0207 & UF & Environmental & 19960708 & 1700 & .84 & .1 & 6.9 & 497 & 13 \\
\hline 0208 & UF & Environmental & 19950720 & 1050 & 6.9 & 5.7 & 7.1 & 605 & 13 \\
\hline 0208 & UF & Environmental & 19960718 & 1320 & 8.4 & 5.9 & 6.7 & 630 & 13 \\
\hline 0209 & UF & Environmental & 19950720 & 1200 & 2.1 & 5.7 & 7.3 & 433 & 13 \\
\hline 0209 & UF & Environmental & 19960718 & 1350 & 2.2 & 8.5 & 7.4 & 357 & 15 \\
\hline 0210 & UF & Environmental & 19950720 & 0955 & 7.1 & 7.6 & 7.0 & 600 & 12.5 \\
\hline 0210 & UF & Environmental & 19960708 & 1610 & 7.2 & 7.5 & 6.8 & 627 & 13.5 \\
\hline 0211 & UF & Environmental & 19950719 & 1330 & 4.2 & 3.7 & 7.3 & 920 & 13.5 \\
\hline 0211 & UF & Environmental & 19960718 & 1245 & 5.3 & 3.6 & 6.8 & 920 & 13 \\
\hline 0212 & UF & Environmental & 19960716 & 1455 & $* 11$ & 7.5 & 7.0 & 634 & 13 \\
\hline 0213 & UF & Environmental & 19950719 & 1245 & 2.2 & 7.7 & 7.6 & 448 & 13.5 \\
\hline 0213 & UF & Environmental & 19960716 & 1605 & 2.6 & 8.4 & 7.3 & 445 & 13 \\
\hline 0214 & UF & Environmental & 19960716 & 1430 & 4.0 & 4.4 & 6.8 & 609 & 13 \\
\hline 0215 & UF & Environmental & 19950719 & 1530 & 6.0 & .8 & 7.4 & 714 & 13.5 \\
\hline 0215 & UF & Environmental & 19960708 & 1435 & $* 13$ & .9 & 6.9 & 820 & 14.5 \\
\hline 0301 & $\mathrm{CF}$ & Environmental & 19950725 & 1245 & 3.2 & 4.4 & 7.2 & 677 & 13.5 \\
\hline 0301 & $\mathrm{CF}$ & Environmental & 19960719 & 1430 & 3.3 & 6.3 & 6.8 & 533 & 14.5 \\
\hline 0302 & UF & Environmental & 19950724 & 1725 & 5.9 & 7.5 & 7.4 & 468 & 13.5 \\
\hline 0302 & UF & Environmental & 19960730 & 1405 & 6.9 & 7.4 & 7.2 & 1,010 & 13 \\
\hline 0303 & UF & Environmental & 19960715 & 1215 & .93 & 2.0 & 6.5 & 522 & 13 \\
\hline 0304 & UF & Environmental & 19950718 & 1700 & 5.6 & 8.0 & 6.8 & 506 & 13.5 \\
\hline 0305 & UF & Environmental & 19950718 & 1610 & 1.7 & 2.1 & 7.0 & 558 & 13 \\
\hline 0305 & UF & Environmental & 19960715 & 1340 & 2.3 & 2.4 & 6.6 & 610 & 13.5 \\
\hline 0306 & UF & Environmental & 19950718 & 1320 & 1.3 & 4.0 & 6.8 & 430 & 13.5 \\
\hline 0306 & UF & Environmental & 19960709 & 1150 & 1.3 & 4.2 & 6.5 & 427 & 13.3 \\
\hline 0306 & UF & Duplicate & 19960709 & 1155 & 1.2 & 4.2 & 6.5 & 427 & 13.5 \\
\hline 0306 & UF & Blank & 19960709 & 1200 & $<.05$ & -- & -- & -- & -- \\
\hline 0307 & UF & Environmental & 19950725 & 0830 & 2.7 & 4.2 & 6.8 & 590 & 13.5 \\
\hline 0307 & UF & Duplicate & 19950725 & 0835 & 2.7 & 4.2 & 6.8 & 590 & 13.5 \\
\hline 0307 & UF & Blank & 19950725 & 0840 & $<.05$ & -- & -- & -- & -- \\
\hline 0307 & UF & Environmental & 19960719 & 1505 & 2.4 & 3.9 & 6.5 & 590 & 13.4 \\
\hline 0308 & UF & Environmental & 19950724 & 1630 & 5.5 & 6.9 & 6.8 & 543 & 12.5 \\
\hline 0308 & UF & Environmental & 19960717 & 1025 & 6.4 & 7.9 & 6.7 & 578 & 12.5 \\
\hline
\end{tabular}


Table 8. Nitrate concentrations and field measurements for water samples from the ground-water-quality monitoring network of domestic and registered wells, Upper Big Blue Natural Resources District, central Nebraska, July 1995 through September 1997--Continued

\begin{tabular}{|c|c|c|c|c|c|c|c|c|c|}
\hline $\begin{array}{c}\text { Well } \\
\text { identification } \\
\text { number }^{1} \\
\text { (fig. } 8 \text { ) }\end{array}$ & $\begin{array}{l}\text { Hydro- } \\
\text { geologic } \\
\text { condition }\end{array}$ & Sample type & $\begin{array}{l}\text { Sample } \\
\text { date }\end{array}$ & $\begin{array}{l}\text { Sample } \\
\text { time }\end{array}$ & $\begin{array}{l}\text { Nitrate and } \\
\text { nitrite as } \\
\text { nitrogen, } \\
\text { dissolved } \\
(\mathrm{mg} / \mathrm{L})\end{array}$ & $\begin{array}{c}\text { Oxygen, } \\
\text { dissolved, } \\
\text { field } \\
(\mathrm{mg} / \mathrm{L})\end{array}$ & $\begin{array}{l}\mathrm{pH}, \\
\text { field } \\
\text { (standard } \\
\text { units) }\end{array}$ & $\begin{array}{c}\text { Specific } \\
\text { conduct- } \\
\text { ance, field } \\
(\mu \mathrm{S} / \mathrm{cm})\end{array}$ & $\begin{array}{c}\text { Water } \\
\text { temperature } \\
\left({ }^{\circ} \mathrm{C}\right)\end{array}$ \\
\hline 0308 & UF & Duplicate & 19960717 & 1030 & 6.1 & 7.9 & 6.7 & 578 & 12.5 \\
\hline 0308 & UF & Blank & 19960717 & 1035 & .07 & - & - & - & - \\
\hline 0309 & $\mathrm{CF}$ & Environmental & 19960717 & 1100 & 1.1 & 7.3 & 6.6 & 571 & 13 \\
\hline 0310 & $\mathrm{CF}$ & Environmental & 19950718 & 1700 & 3.3 & 7.4 & 7.2 & 660 & 14 \\
\hline 0310 & $\mathrm{CF}$ & Environmental & 19960715 & 1520 & 3.7 & 7.1 & 6.6 & 638 & 13.5 \\
\hline 0311 & UF & Environmental & 19950717 & 1530 & 1.6 & 7.2 & 7.0 & 860 & 13 \\
\hline 0311 & UF & Environmental & 19960716 & 1125 & 1.8 & 8.7 & 6.6 & 871 & 12.5 \\
\hline 0312 & UF & Environmental & 19950717 & 1615 & 9.5 & 7.6 & 7.0 & 735 & 13.5 \\
\hline 0312 & UF & Environmental & 19960716 & 1215 & $* 11$ & 7.1 & 6.8 & 734 & 13 \\
\hline 0313 & CF & Environmental & 19950725 & 1010 & 1.0 & 2.6 & 7.4 & 436 & 13.5 \\
\hline 0313 & $\mathrm{CF}$ & Environmental & 19960716 & 0950 & 1.0 & 2.5 & 6.9 & 441 & 13.5 \\
\hline 0314 & CF & Environmental & 19950717 & 1430 & 4.0 & 6.7 & 7.2 & 766 & 13.5 \\
\hline 0315 & $\mathrm{CF}$ & Environmental & 19950725 & 1120 & 1.5 & .3 & 7.2 & 695 & 17 \\
\hline 0315 & $\mathrm{CF}$ & Environmental & 19960716 & 0905 & .92 & .1 & 6.8 & 675 & 15.5 \\
\hline 0316 & $\mathrm{CF}$ & Environmental & 19960718 & 1000 & 5.0 & 5.6 & 6.8 & 600 & 14 \\
\hline 0317 & UF & Environmental & 19950721 & 830 & 2.2 & 3.8 & 7.0 & 627 & 13.5 \\
\hline 0317 & UF & Environmental & 19960717 & 1425 & 3.3 & 4.5 & 6.7 & 642 & 13 \\
\hline 0317 & UF & Environmental & 19960718 & 0855 & 4.5 & 3.0 & 6.6 & 651 & 13 \\
\hline 0318 & UF & Environmental & 19950727 & 1045 & 4.3 & 3.3 & 6.9 & 652 & 13 \\
\hline 0319 & CF & Environmental & 19950725 & 1555 & 4.9 & 7.2 & 7.2 & 592 & 13 \\
\hline 0320 & UF & Environmental & 19950719 & 1000 & .72 & .6 & 7.0 & 831 & 12.5 \\
\hline 0320 & UF & Environmental & 19960715 & 1625 & .40 & .1 & 6.7 & 851 & 12.5 \\
\hline 0321 & UF & Environmental & 19950719 & 1055 & $*<.05$ & 6.4 & 7.3 & 694 & 12.5 \\
\hline 0321 & UF & Environmental & 19960716 & 1345 & 9.6 & 4.3 & 6.7 & 570 & 14 \\
\hline 0321 & UF & Environmental & 19970729 & 1700 & 6.2 & 6.3 & 7.1 & 632 & 12.5 \\
\hline 0401 & $\mathrm{CF}$ & Environmental & 19950720 & 0815 & 4.5 & 8.3 & 7.0 & 553 & 12 \\
\hline 0401 & $\mathrm{CF}$ & Environmental & 19960709 & 1347 & 5.1 & 7.9 & 7.0 & 560 & 12 \\
\hline 0402 & UF & Environmental & 19950720 & 1053 & 9.7 & 7.0 & 6.9 & 678 & 12.5 \\
\hline 0402 & UF & Environmental & 19960709 & 1247 & *12 & 8.2 & 6.9 & 717 & 13 \\
\hline 0402 & UF & Blank & 19960709 & 1252 & .06 & -- & -- & - & - \\
\hline 0402 & UF & Duplicate & 19960709 & 1257 & $* 11$ & 6.9 & 6.9 & 717 & 13 \\
\hline 0403 & UF & Environmental & 19950720 & 1125 & 1.6 & 4.8 & 6.8 & 543 & 13 \\
\hline 0403 & UF & Environmental & 19960709 & 1429 & 1.6 & 4.7 & 7.0 & 538 & 13 \\
\hline 0404 & UF & Environmental & 19950719 & 1506 & 6.5 & 5.9 & 6.9 & 590 & 13.5 \\
\hline 0404 & UF & Environmental & 19950719 & 1733 & 3.7 & 7.2 & 7.4 & 511 & 12.5 \\
\hline 0404 & UF & Environmental & 19960709 & 1512 & 3.9 & 6.1 & 7.1 & 572 & 13.5 \\
\hline 0405 & UF & Environmental & 19950719 & 1509 & 6.5 & 5.9 & 6.9 & 590 & 13.5 \\
\hline 0406 & $\mathrm{CF}$ & Environmental & 19950724 & 1800 & 3.4 & 3.7 & 6.8 & 561 & 12 \\
\hline 0406 & CF & Environmental & 19950726 & 1500 & 3.4 & 3.9 & 7.0 & 554 & 12.5 \\
\hline 0406 & CF & Environmental & 19960709 & 1655 & 3.0 & 3.4 & 6.9 & 554 & 12.5 \\
\hline 0407 & UF & Environmental & 19950720 & 1220 & 8.3 & 7.3 & 6.8 & 613 & 13.5 \\
\hline 0407 & UF & Environmental & 19960709 & 1157 & 9.2 & 7.8 & 7.0 & 628 & 13 \\
\hline 0408 & UF & Environmental & 19950719 & 1655 & 1.9 & 2.0 & 7.0 & 528 & 13 \\
\hline 0408 & UF & Environmental & 19960710 & 0832 & 2.0 & 2.2 & 7.2 & 587 & 12 \\
\hline 0409 & UF & Environmental & 19960709 & 1004 & $* 11$ & 7.3 & 7.1 & 741 & 15 \\
\hline
\end{tabular}


Table 8. Nitrate concentrations and field measurements for water samples from the ground-water-quality monitoring network of domestic and registered wells, Upper Big Blue Natural Resources District, central Nebraska, July 1995 through September 1997--Continued

\begin{tabular}{|c|c|c|c|c|c|c|c|c|c|}
\hline $\begin{array}{c}\text { Well } \\
\text { identification } \\
\text { number }{ }^{1} \\
\text { (fig. } 8 \text { ) }\end{array}$ & $\begin{array}{l}\text { Hydro- } \\
\text { geologic } \\
\text { condition }\end{array}$ & Sample type & $\begin{array}{c}\text { Sample } \\
\text { date }\end{array}$ & $\begin{array}{c}\text { Sample } \\
\text { time }\end{array}$ & $\begin{array}{l}\text { Nitrate and } \\
\text { nitrite as } \\
\text { nitrogen, } \\
\text { dissolved } \\
(\mathrm{mg} / \mathrm{L})\end{array}$ & $\begin{array}{l}\text { Oxygen, } \\
\text { dissolved, } \\
\text { field } \\
(\mathrm{mg} / \mathrm{L})\end{array}$ & $\begin{array}{c}\mathrm{pH}, \\
\text { field } \\
\text { (standard } \\
\text { units) }\end{array}$ & $\begin{array}{c}\text { Specific } \\
\text { conduct- } \\
\text { ance, field } \\
(\mu \mathrm{S} / \mathrm{cm})\end{array}$ & $\begin{array}{c}\text { Water } \\
\text { temperature } \\
\left({ }^{\circ} \mathrm{C}\right)\end{array}$ \\
\hline 0410 & $\mathrm{CF}$ & Environmental & 19950726 & 1345 & $<.05$ & 6.3 & 7.5 & 502 & 14 \\
\hline 0410 & $\mathrm{CF}$ & Environmental & 19960710 & 0958 & .23 & 6.5 & 7.5 & 511 & 14 \\
\hline 0411 & $\mathrm{CF}$ & Environmental & 19960719 & 1040 & 1.5 & .9 & 7.1 & 674 & 14 \\
\hline 0412 & UF & Environmental & 19960710 & 1047 & *15 & 6.9 & 7.0 & 718 & 12.5 \\
\hline 0413 & UF & Environmental & 19950724 & 1055 & 8.4 & 5.0 & 7.1 & 616 & 12 \\
\hline 0413 & UF & Environmental & 19960715 & 1143 & 4.1 & 4.6 & 7.1 & 639 & 12.5 \\
\hline 0414 & UF & Environmental & 19950728 & 1620 & 3.4 & 3.4 & 6.8 & 454 & 13.5 \\
\hline 0414 & UF & Environmental & 19960715 & 1103 & 4.2 & 4.8 & 6.9 & 468 & 13 \\
\hline 0415 & UF & Environmental & 19950724 & 0810 & 5.6 & 8.5 & 6.7 & 601 & 12.5 \\
\hline 0415 & UF & Environmental & 19960710 & 1125 & 8.2 & 8.4 & 7.0 & 668 & 13 \\
\hline 0416 & UF & Environmental & 19960711 & 1234 & 8.7 & 17 & 7.1 & 682 & 13 \\
\hline 0417 & $\mathrm{CF}$ & Blank & 19960711 & 1414 & .06 & -- & -- &.- & -- \\
\hline 0417 & $\mathrm{CF}$ & Duplicate & 19960711 & 1415 & $* 18$ & 5.1 & 7.1 & 823 & 12 \\
\hline 0417 & $\mathrm{CF}$ & Environmental & 19960711 & 1416 & $* 18$ & 5.0 & 7.1 & 823 & 12 \\
\hline 0418 & $\mathrm{CF}$ & Environmental & 19960711 & 1107 & 4.6 & 5.8 & 7.1 & 736 & 13 \\
\hline 0419 & $\mathrm{CF}$ & Environmental & 19960711 & 1319 & 6.0 & 9.3 & 7.0 & 473 & 12.5 \\
\hline 0420 & UF & Environmental & 19950724 & 0950 & 5.5 & 9.5 & 7.3 & 527 & 13 \\
\hline 0420 & UF & Environmental & 19960719 & 1139 & 7.1 & 8.8 & 6.8 & 576 & 14 \\
\hline 0501 & $\mathrm{CF}$ & Environmental & 19950725 & 1721 & .18 & .3 & 7.3 & 550 & 13.5 \\
\hline 0501 & $\mathrm{CF}$ & Duplicate & 19950725 & 1726 & .17 & .3 & 7.3 & 550 & 13.5 \\
\hline 0501 & $\mathrm{CF}$ & Blank & 19950725 & 1731 & $<.05$ & -- & -- & -- & -- \\
\hline 0501 & $\mathrm{CF}$ & Environmental & 19960716 & 0833 & .25 & .8 & 7.4 & 560 & 14 \\
\hline 0502 & UF & Environmental & 19960712 & 1445 & $* 12$ & 6.0 & 6.4 & 456 & 13 \\
\hline 0503 & UF & Environmental & 19950726 & 1359 & $* 27$ & 5.3 & 6.2 & 616 & 14 \\
\hline 0503 & UF & Environmental & 19960709 & 0911 & $* 27$ & 6.5 & 6.4 & 802 & 12 \\
\hline 0504 & UF & Environmental & 19960712 & 0915 & 8.7 & 8.9 & 6.8 & 620 & 12.5 \\
\hline 0504 & UF & Duplicate & 19960712 & 0920 & 9.3 & 8.9 & 6.8 & 620 & 12.5 \\
\hline 0504 & UF & Blank & 19960712 & 0925 & $<.05$ & -- & -. & -- & -- \\
\hline 0505 & UF & Environmental & 19950717 & 1529 & $* 12$ & 7.8 & 6.7 & 646 & 13.5 \\
\hline 0505 & UF & Environmental & 19960709 & 1042 & $* 13$ & 9.0 & 7.1 & 647 & 12 \\
\hline 0506 & UF & Environmental & 19950830 & 1615 & $* 13$ & 8.4 & 7.2 & 560 & 13 \\
\hline 0507 & UF & Environmental & 19950720 & 1305 & 6.2 & 8.7 & 6.9 & 611 & 12 \\
\hline 0507 & UF & Environmental & 19960708 & 1600 & 6.0 & 7.4 & 7.1 & 603 & 13 \\
\hline 0508 & UF & Environmental & 19950720 & 1345 & 4.2 & 5.0 & 7.0 & 571 & 13.5 \\
\hline 0508 & UF & Blank & 19950720 & 1350 & $<0.05$ & - & - & - & -- \\
\hline 0508 & UF & Duplicate & 19950720 & 1355 & 4.2 & 5.0 & 7.0 & 571 & 13.5 \\
\hline 0508 & UF & Environmental & 19960708 & 1518 & 5.4 & 6.4 & 7.0 & 426 & 13.5 \\
\hline 0509 & $\mathrm{CF}$ & Environmental & 19960708 & 1414 & 3.7 & .8 & 7.4 & 482 & 15 \\
\hline 0510 & $\mathrm{CF}$ & Environmental & 19950721 & 1420 & $* 11$ & 10 & 7.5 & 782 & 12.5 \\
\hline 0510 & $\mathrm{CF}$ & Environmental & 19960709 & 0953 & $* 12$ & 9.6 & 7.1 & 772 & 12.5 \\
\hline 0511 & UF & Environmental & 19950720 & 1425 & $* 18$ & 9.4 & 7.4 & 837 & 12.5 \\
\hline 0511 & UF & Environmental & 19960715 & 1712 & *18 & 7.8 & 7.2 & 865 & 13.5 \\
\hline 0512 & UF & Environmental & 19950829 & 1430 & 4.8 & 5.0 & 7.1 & 550 & 14 \\
\hline 0512 & UF & Environmental & 19960715 & 1628 & 8.9 & 6.7 & 7.1 & 646 & 13 \\
\hline
\end{tabular}


Table 8. Nitrate concentrations and field measurements for water samples from the ground-water-quality monitoring network of domestic and registered wells, Upper Big Blue Natural Resources District, central Nebraska, July 1995 through September 1997--Continued

\begin{tabular}{|c|c|c|c|c|c|c|c|c|c|}
\hline $\begin{array}{c}\text { Well } \\
\text { identification } \\
\text { number }^{1} \\
\text { (fig. } 8 \text { ) }\end{array}$ & $\begin{array}{l}\text { Hydro- } \\
\text { geologic } \\
\text { condition }\end{array}$ & Sample type & $\begin{array}{c}\text { Sample } \\
\text { date }\end{array}$ & $\begin{array}{c}\text { Sample } \\
\text { time }\end{array}$ & $\begin{array}{l}\text { Nitrate and } \\
\text { nitrite as } \\
\text { nitrogen, } \\
\text { dissolved } \\
(\mathrm{mg} / \mathrm{L})\end{array}$ & $\begin{array}{c}\text { Oxygen, } \\
\text { dissolved, } \\
\text { field } \\
(\mathrm{mg} / \mathrm{L})\end{array}$ & $\begin{array}{c}\mathrm{pH}, \\
\text { field } \\
\text { (standard } \\
\text { units) }\end{array}$ & $\begin{array}{c}\text { Specific } \\
\text { conduct- } \\
\text { ance, field } \\
(\mu \mathrm{S} / \mathrm{cm})\end{array}$ & $\begin{array}{c}\text { Water } \\
\text { temperature } \\
\left({ }^{\circ} \mathrm{C}\right)\end{array}$ \\
\hline 0512 & UF & Blank & 19960715 & 1633 & .05 & - & - & -- & - \\
\hline 0512 & UF & Duplicate & 19960715 & 1638 & 8.7 & 6.7 & 7.1 & 646 & 13 \\
\hline 0601 & UF & Environmental & 19950728 & 1590 & 4.5 & 6.9 & 7.0 & 534 & 12 \\
\hline 0602 & UF & Environmental & 19950727 & 0938 & 7.1 & 9.1 & 7.2 & 785 & 14 \\
\hline 0602 & UF & Environmental & 19960716 & 1100 & $* 14$ & 7.2 & 6.9 & 775 & 12.5 \\
\hline 0603 & $\mathrm{CF}$ & Environmental & 19950829 & 1340 & 2.9 & 7.5 & 7.3 & 480 & 14.5 \\
\hline 0603 & $\mathrm{CF}$ & Environmental & 19960814 & 1000 & 3.1 & 9.8 & 7.1 & 566 & 13 \\
\hline 0604 & UF & Environmental & 19950724 & 1117 & 6.0 & 6.4 & 7.1 & 590 & 13 \\
\hline 0604 & UF & Environmental & 19960716 & 1237 & 8.0 & 7.1 & 7.0 & 686 & 13.1 \\
\hline 0605 & UF & Environmental & 19950724 & 1148 & 6.2 & 8.0 & 7.6 & 576 & 12.5 \\
\hline 0605 & UF & Environmental & 19960712 & 1040 & 7.1 & 8.9 & 6.9 & 579 & 12.5 \\
\hline 0606 & UF & Environmental & 19950724 & 1234 & 7.4 & 8.8 & 7.0 & 637 & 13 \\
\hline 0606 & UF & Environmental & 19960716 & 1502 & $* 10$ & 7.4 & 7.0 & 644 & 13.7 \\
\hline 0607 & UF & Environmental & 19960716 & 1207 & $* 13$ & 9.4 & 7.5 & 676 & 13 \\
\hline 0608 & UF & Environmental & 19950724 & 1345 & 5.6 & 2.5 & 6.9 & 609 & 12.5 \\
\hline 0608 & UF & Environmental & 19960712 & 1115 & 6.2 & 2.1 & 6.8 & 606 & 13 \\
\hline 0609 & UF & Environmental & 19960717 & 1445 & 1.7 & 4.5 & 6.6 & 548 & 13 \\
\hline 0610 & UF & Environmental & 19950719 & 1800 & 2.1 & 4.6 & 6.8 & 555 & 13.5 \\
\hline 0610 & UF & Environmental & 19960719 & 1550 & 2.0 & 3.8 & 6.5 & 544 & 14 \\
\hline 0610 & UF & Duplicate & 19960719 & 1555 & 1.8 & 3.8 & 6.5 & 544 & 14 \\
\hline 0610 & UF & Blank & 19960719 & 1600 & .12 & - & - & -- & -- \\
\hline 0611 & UF & Environmental & 19950718 & 1755 & $* 10$ & 13 & 7.1 & 715 & 12.5 \\
\hline 0611 & UF & Environmental & 19960715 & 1735 & $* 15$ & 11 & 7.3 & 752 & 13 \\
\hline 0612 & UF & Environmental & 19960712 & 955 & 6.2 & 7.1 & 6.8 & 591 & 12 \\
\hline 0613 & UF & Environmental & 19950724 & 1450 & 4.5 & 6.7 & 7.1 & 548 & 13.5 \\
\hline 0613 & UF & Environmental & 19960716 & 1334 & 4.5 & 5.9 & 7.1 & 550 & 13 \\
\hline 0614 & UF & Environmental & 19960716 & 1710 & $* 12$ & 7.3 & 6.7 & 694 & 12 \\
\hline 0615 & $\mathrm{CF}$ & Environmental & 19950724 & 1419 & 6.8 & 6.9 & 7.1 & 587 & 13.5 \\
\hline 0615 & CF & Environmental & 19950727 & 1220 & 4.6 & 5.9 & 6.8 & 503 & 13.5 \\
\hline 0615 & $\mathrm{CF}$ & Environmental & 19960813 & 0930 & 5.4 & 7.8 & 6.7 & 551 & 13 \\
\hline 0701 & CF & Environmental & 19950719 & 1032 & .73 & .1 & 7.3 & 414 & 13.5 \\
\hline 0701 & $\mathrm{CF}$ & Environmental & 19960709 & 1630 & 1.5 & 0.2 & 7.0 & 479 & 13.5 \\
\hline 0701 & CF & Environmental & 19960717 & 1431 & 1.1 & .2 & 7.2 & 470 & 14.5 \\
\hline 0702 & $\mathrm{CF}$ & Environmental & 19960717 & 1218 & 6.2 & 4.0 & 7.1 & 614 & 13.5 \\
\hline 0703 & $\mathrm{CF}$ & Environmental & 19960730 & 1140 & 4.7 & 1.5 & 7.1 & 1,490 & 13 \\
\hline 0704 & $\mathrm{CF}$ & Environmental & 19950721 & 0830 & .12 & .3 & 7.0 & 398 & 14 \\
\hline 0704 & $\mathrm{CF}$ & Environmental & 19960710 & 1125 & .96 & .5 & 6.6 & 401 & 14 \\
\hline 0705 & $\mathrm{CF}$ & Environmental & 19960718 & 1008 & .12 & .2 & 7.3 & 488 & 14 \\
\hline 0706 & CF & Environmental & 19950725 & 1500 & .12 & - & 7.2 & 690 & 13.5 \\
\hline 0706 & CF & Environmental & 19960709 & 1550 & $* 6.8$ & $<.1$ & 6.8 & 677 & 13 \\
\hline 0706 & CF & Environmental & 19960718 & 1543 & .37 & .2 & 7.1 & 427 & 14 \\
\hline 0706 & CF & Environmental & 19970729 & 1240 & .16 & .6 & 7.0 & 669 & 13 \\
\hline 0707 & $\mathrm{CF}$ & Environmental & 19950719 & 1207 & 1.4 & .2 & 7.1 & 646 & 14 \\
\hline 0707 & $\mathrm{CF}$ & Blank & 19950719 & 1212 & $<.05$ & -- & -- & -- & -- \\
\hline
\end{tabular}


Table 8. Nitrate concentrations and field measurements for water samples from the ground-water-quality monitoring network of domestic and registered wells, Upper Big Blue Natural Resources District, central Nebraska, July 1995 through September 1997--Continued

\begin{tabular}{|c|c|c|c|c|c|c|c|c|c|}
\hline $\begin{array}{c}\text { Well } \\
\text { identification } \\
\text { number }^{1} \\
\text { (fig. } 8 \text { ) }\end{array}$ & $\begin{array}{l}\text { Hydro- } \\
\text { geologic } \\
\text { condition }\end{array}$ & Sample type & $\begin{array}{c}\text { Sample } \\
\text { date }\end{array}$ & $\begin{array}{l}\text { Sample } \\
\text { time }\end{array}$ & $\begin{array}{l}\text { Nitrate and } \\
\text { nitrite as } \\
\text { nitrogen, } \\
\text { dissolved } \\
\text { (mg/L) }\end{array}$ & $\begin{array}{l}\text { Oxygen, } \\
\text { dissolved, } \\
\text { field } \\
(\mathrm{mg} / \mathrm{L})\end{array}$ & $\begin{array}{c}\mathrm{pH}, \\
\text { field } \\
\text { (standard } \\
\text { units) }\end{array}$ & $\begin{array}{c}\text { Specific } \\
\text { conduct- } \\
\text { ance, field } \\
(\mu \mathrm{S} / \mathrm{cm})\end{array}$ & $\begin{array}{c}\text { Water } \\
\text { temperature } \\
\left({ }^{\circ} \mathrm{C}\right)\end{array}$ \\
\hline 0707 & $\mathrm{CF}$ & Duplicate & 19950719 & 1217 & 1.4 & .2 & 7.1 & 646 & 14 \\
\hline 0707 & CF & Environmental & 19960709 & 1515 & 3.6 & .5 & 6.6 & 964 & 13.5 \\
\hline 0707 & $\mathrm{CF}$ & Environmental & 19960717 & 1602 & 2.0 & 5.6 & 7.1 & 777 & 15 \\
\hline 0708 & $\mathrm{CF}$ & Environmental & 19950726 & 1617 & 2.5 & .1 & 7.0 & 701 & 13.5 \\
\hline 0708 & CF & Environmental & 19960717 & 1513 & 3.4 & .2 & 7.1 & 726 & 15 \\
\hline 0709 & $\mathrm{CF}$ & Environmental & 19950721 & 1622 & 6.2 & 14 & 7.1 & 647 & 13 \\
\hline 0709 & CF & Blank & 19950721 & 1627 & $<.05$ & -- & - & -- & -- \\
\hline 0709 & $\mathrm{CF}$ & Duplicate & 19950721 & 1632 & 6.1 & 14 & 7.1 & 647 & 13 \\
\hline 0709 & $\mathrm{CF}$ & Environmental & 19960717 & 1705 & $* 11$ & 3.0 & 7.0 & 729 & 13.5 \\
\hline 0709 & $\mathrm{CF}$ & Environmental & 19970722 & 1503 & 7.2 & 2.5 & 6.8 & 609 & 12 \\
\hline 0710 & $\mathrm{CF}$ & Environmental & 19960719 & 1535 & 6.0 & 2.9 & 7.1 & 1,300 & 13 \\
\hline 0711 & $\mathrm{CF}$ & Environmental & 19960718 & 841 & 2.4 & 6.7 & 7.4 & 553 & 14.6 \\
\hline 0712 & $\mathrm{CF}$ & Environmental & 19950725 & 1325 & 1.5 & .4 & 7.1 & 521 & 13 \\
\hline 0712 & $\mathrm{CF}$ & Environmental & 19960710 & 0900 & 1.3 & .4 & 6.9 & 538 & 13 \\
\hline 0713 & CF & Environmental & 19960710 & 0825 & $* 12$ & 1.6 & 6.7 & 761 & 13.5 \\
\hline 0714 & CF & Environmental & 19950721 & 1435 & $<.05$ & .1 & 7.1 & 500 & 15 \\
\hline 0714 & $\mathrm{CF}$ & Blank & 19960719 & 1616 & .07 & - & - & - & - \\
\hline 0714 & $\mathrm{CF}$ & Environmental & 19960719 & 1621 & .10 & .4 & 7.1 & 504 & 15 \\
\hline 0714 & $\mathrm{CF}$ & Duplicate & 19960719 & 1626 & .10 & .4 & 7.1 & 504 & 15 \\
\hline 0715 & UF & Environmental & 19960719 & 1433 & 6.3 & 7.4 & 6.9 & 590 & 14 \\
\hline 0716 & UF & Environmental & 19950726 & 1515 & 4.5 & 6.8 & 6.9 & 762 & 13.5 \\
\hline 0716 & UF & Environmental & 19960716 & 1128 & 4.6 & 6.8 & 7.0 & 738 & 12.5 \\
\hline 0717 & $\mathrm{CF}$ & Environmental & 19950725 & 1635 & 2.7 & 1.4 & 7.4 & 605 & 13.5 \\
\hline 0717 & $\mathrm{CF}$ & Environmental & 19960716 & 1020 & $* 18$ & 8.6 & 7.0 & 837 & 12.5 \\
\hline 0717 & $\mathrm{CF}$ & Environmental & 19970722 & 1401 & 6.8 & 4.9 & 6.9 & 641 & 12.5 \\
\hline 0718 & UF & Environmental & 19950724 & 0833 & 7.0 & 10 & 8.0 & 615 & 13.5 \\
\hline 0718 & UF & Environmental & 19960712 & 1215 & 7.3 & 9.0 & 6.9 & 609 & 13 \\
\hline 0719 & UF & Environmental & 19950717 & 1728 & 2.8 & 6.7 & 6.9 & 430 & 13 \\
\hline 0719 & UF & Environmental & 19960712 & 1405 & 3.1 & 7.7 & 6.5 & 424 & 13 \\
\hline 0801 & UF & Environmental & 19950726 & 1024 & 2.9 & 4.5 & 7.0 & 490 & 12.5 \\
\hline 0801 & UF & Environmental & 19960709 & 1710 & 2.6 & 5.1 & 6.7 & 535 & 12.5 \\
\hline 0801 & UF & Environmental & 19960718 & 1338 & 5.0 & 5.6 & 7.1 & 392 & 13 \\
\hline 0802 & UF & Environmental & 19950718 & 0905 & $* 11$ & 6.1 & 7.0 & 788 & 12.5 \\
\hline 0802 & UF & Environmental & 19960718 & 1403 & 9.3 & 7.0 & 7.0 & 487 & 13 \\
\hline 0803 & UF & Environmental & 19960710 & 1520 & 2.6 & .9 & 6.5 & 781 & 12.5 \\
\hline 0804 & CF & Environmental & 19960717 & 1020 & .13 & 1.0 & 7.2 & 555 & 13.5 \\
\hline 0805 & $\mathrm{CF}$ & Environmental & 19950830 & 1415 & .14 & .4 & 7.4 & 520 & 13 \\
\hline 0806 & CF & Environmental & 19950718 & 1053 & .66 & .1 & 7.3 & 557 & 13 \\
\hline 0806 & CF & Blank & 19950718 & 1058 & $<.05$ & -- & - & - & -- \\
\hline 0806 & $\mathrm{CF}$ & Duplicate & 19950718 & 1103 & .75 & .1 & 7.3 & 557 & 13 \\
\hline 0806 & $\mathrm{CF}$ & Environmental & 19950718 & 1340 & 2.0 & .2 & 7.0 & 677 & 13 \\
\hline 0806 & CF & Environmental & 19960710 & 1300 & 1.5 & .1 & 6.8 & 583 & 13 \\
\hline 0806 & $\mathrm{CF}$ & Duplicate & 19960710 & 1305 & 1.4 & .1 & 6.8 & 583 & 13 \\
\hline 0806 & $\mathrm{CF}$ & Blank & 19960710 & 1310 & $<.05$ & -- & -- & -- & -- \\
\hline
\end{tabular}


Table 8. Nitrate concentrations and field measurements for water samples from the ground-water-quality monitoring network of domestic and registered wells, Upper Big Blue Natural Resources District, central Nebraska, July 1995 through September 1997--Continued

\begin{tabular}{|c|c|c|c|c|c|c|c|c|c|}
\hline $\begin{array}{c}\text { Well } \\
\text { identification } \\
\text { number }^{1} \\
\text { (fig. 8) }\end{array}$ & $\begin{array}{l}\text { Hydro- } \\
\text { geologic } \\
\text { condition }\end{array}$ & Sample type & $\begin{array}{c}\text { Sample } \\
\text { date }\end{array}$ & $\begin{array}{c}\text { Sample } \\
\text { time }\end{array}$ & $\begin{array}{c}\text { Nitrate and } \\
\text { nitrite as } \\
\text { nitrogen, } \\
\text { dissolved } \\
(\mathrm{mg} / \mathrm{L})\end{array}$ & $\begin{array}{l}\text { Oxygen, } \\
\text { dissolved, } \\
\text { field } \\
(\mathrm{mg} / \mathrm{L})\end{array}$ & $\begin{array}{c}\mathrm{pH}, \\
\text { field } \\
\text { (standard } \\
\text { units) }\end{array}$ & $\begin{array}{c}\text { Specific } \\
\text { conduct- } \\
\text { ance, field } \\
(\mu \mathrm{S} / \mathrm{cm})\end{array}$ & $\begin{array}{c}\text { Water } \\
\text { temperature } \\
\left({ }^{\circ} \mathrm{C}\right)\end{array}$ \\
\hline 0806 & $\mathrm{CF}$ & Environmental & 19960710 & 1350 & 2.7 & .2 & 6.8 & 690 & 12.5 \\
\hline 0807 & CF & Environmental & 19950718 & 1230 & $<.05$ & .1 & 7.1 & 616 & 13 \\
\hline 0807 & $\mathrm{CF}$ & Environmental & 19960717 & 0857 & .12 & .5 & 7.2 & 616 & 14 \\
\hline 0808 & UF & Environmental & 19950727 & 1217 & 2.5 & 2.9 & 7.0 & 558 & 13.5 \\
\hline 0808 & UF & Environmental & 19960718 & 1048 & 3.6 & 3.2 & 7.2 & 583 & 13 \\
\hline 0809 & UF & Environmental & 19950718 & 1430 & 8.0 & 6.8 & 6.8 & 550 & 13 \\
\hline 0809 & UF & Environmental & 19960718 & 1137 & $* 16$ & 8.4 & 6.8 & 655 & 13.5 \\
\hline 0809 & UF & Blank & 19960718 & 1142 & .08 & -- & -- & -- & -- \\
\hline 0809 & UF & Duplicate & 19960718 & 1147 & $* 16$ & 8.4 & 6.8 & 655 & 13.5 \\
\hline 0809 & UF & Environmental & 19970722 & 1620 & $* 10$ & 8.2 & 6.9 & 560 & 12 \\
\hline 0810 & UF & Environmental & 19950718 & 1550 & 3.5 & 9.3 & 7.4 & 500 & 13.5 \\
\hline 0811 & $\mathrm{CF}$ & Environmental & 19950727 & 1510 & 4.4 & 6.4 & 7.8 & 591 & 13 \\
\hline 0811 & CF & Environmental & 19960711 & 1320 & 4.8 & 4.6 & 6.8 & 590 & 12.5 \\
\hline 0812 & UF & Environmental & 19950801 & 0830 & 5.3 & .4 & 7.1 & 907 & 13.5 \\
\hline 0812 & UF & Environmental & 19960718 & 1231 & 5.6 & .1 & 6.9 & 915 & 13.5 \\
\hline 0813 & UF & Environmental & 19950718 & 1755 & 2.2 & 1.9 & 7.1 & 539 & 13 \\
\hline 0813 & $\mathrm{CF}$ & Environmental & 19960709 & 0915 & 1.7 & 1.7 & 7.0 & 533 & 13 \\
\hline 0814 & UF & Environmental & 19950718 & 1045 & .09 & .3 & 7.2 & 501 & 13.5 \\
\hline 0814 & UF & Environmental & 19960709 & 0945 & .10 & .1 & 7.2 & 503 & 13.5 \\
\hline 0815 & UF & Environmental & 19950718 & 1145 & 1.0 & 3.7 & 6.8 & 335 & 13.5 \\
\hline 0815 & UF & Environmental & 19960709 & 1030 & 1.1 & 3.8 & 6.9 & 317 & 13 \\
\hline 0816 & $\mathrm{CF}$ & Environmental & 19950725 & 1435 & 3.9 & 6.9 & 7.6 & 612 & 14.5 \\
\hline 0816 & $\mathrm{CF}$ & Environmental & 19960719 & 1050 & 4.1 & 5.6 & 6.9 & 597 & 14 \\
\hline 0817 & UF & Environmental & 19960719 & 1135 & .38 & .5 & 6.9 & 645 & 14 \\
\hline 0901 & $\mathrm{CF}$ & Environmental & 19950725 & 0930 & 1.4 & 1.9 & - & 581 & - \\
\hline 0901 & $\mathrm{CF}$ & Environmental & 19960730 & 1130 & 0.4 & 0.6 & 7.2 & 579 & 14 \\
\hline 0902 & UF & Environmental & 19960719 & 1030 & .50 & .7 & 7.3 & 646 & 12.5 \\
\hline 0902 & UF & Environmental & 19970731 & 1425 & .31 & 1.1 & 7.0 & 556 & 12 \\
\hline 0903 & UF & Environmental & 19960719 & 1200 & $* 12$ & 7.6 & 7.2 & 690 & 12 \\
\hline 0904 & $\mathrm{CF}$ & Environmental & 19950725 & 1130 & 8.2 & 4.3 & -- & 627 & -- \\
\hline 0905 & $\mathrm{CF}$ & Environmental & 19960730 & 1200 & 6.0 & - & 6.9 & 622 & 12.5 \\
\hline 0905 & $\mathrm{CF}$ & Environmental & 19960904 & 1400 & 9.9 & 6.7 & 6.9 & 580 & 13 \\
\hline 0906 & UF & Environmental & 19950727 & 1030 & $<.05$ & $<.1$ & 7.3 & 529 & 12.5 \\
\hline 0906 & UF & Duplicate & 19950727 & 1035 & $<.05$ & $<.1$ & 7.3 & 529 & 12.5 \\
\hline 0906 & UF & Blank & 19950727 & 1040 & $<.05$ & -- & - & -- & - \\
\hline 0906 & UF & Environmental & 19960722 & 1230 & .09 & .6 & 7.1 & 526 & 12.5 \\
\hline 0907 & $\mathrm{CF}$ & Environmental & 19950727 & 0930 & .08 & .2 & 7.4 & 550 & 13.5 \\
\hline 0907 & $\mathrm{CF}$ & Environmental & 19960730 & 1600 & 3.6 & 2.5 & 7.1 & 689 & 12.5 \\
\hline 0908 & $\mathrm{CF}$ & Environmental & 19950725 & 1230 & .15 & $<.1$ & 7.3 & 555 & 13 \\
\hline 0909 & $\mathrm{CF}$ & Environmental & 19950726 & 1230 & 1.6 & .6 & 7.0 & 931 & 12.5 \\
\hline 0909 & $\mathrm{CF}$ & Environmental & 19960730 & 1330 & 1.9 & .8 & 6.9 & 925 & 12.5 \\
\hline 0910 & UF & Environmental & 19950726 & 1400 & 4.2 & 2.6 & 6.9 & 693 & 12.5 \\
\hline 0910 & UF & Environmental & 19960722 & 1200 & 6.9 & 2.4 & 6.9 & 701 & 12.5 \\
\hline 0911 & $\mathrm{CF}$ & Environmental & 19950726 & 1300 & 1.2 & 3.3 & 6.9 & 533 & 13 \\
\hline
\end{tabular}


Table 8. Nitrate concentrations and field measurements for water samples from the ground-water-quality monitoring network of domestic and registered wells, Upper Big Blue Natural Resources District, central Nebraska, July 1995 through September 1997--Continued

\begin{tabular}{|c|c|c|c|c|c|c|c|c|c|}
\hline $\begin{array}{c}\text { Well } \\
\text { identification } \\
\text { number }^{1} \\
\text { (fig. } 8 \text { ) }\end{array}$ & $\begin{array}{l}\text { Hydro- } \\
\text { geologic } \\
\text { condition }\end{array}$ & Sample type & $\begin{array}{l}\text { Sample } \\
\text { date }\end{array}$ & $\begin{array}{c}\text { Sample } \\
\text { time }\end{array}$ & $\begin{array}{l}\text { Nitrate and } \\
\text { nitrite as } \\
\text { nitrogen, } \\
\text { dissolved } \\
\text { (mg/L) }\end{array}$ & $\begin{array}{c}\text { Oxygen, } \\
\text { dissolved, } \\
\text { field } \\
(\mathrm{mg} / \mathrm{L})\end{array}$ & $\begin{array}{c}\mathrm{pH}, \\
\text { field } \\
\text { (standard } \\
\text { units) }\end{array}$ & $\begin{array}{c}\text { Specific } \\
\text { conduct- } \\
\text { ance, field } \\
(\mu \mathrm{S} / \mathrm{cm})\end{array}$ & $\begin{array}{c}\text { Water } \\
\text { temperature } \\
\left({ }^{\circ} \mathrm{C}\right)\end{array}$ \\
\hline 0911 & $\mathrm{CF}$ & Environmental & 19960730 & 1300 & 1.5 & 3.2 & 6.8 & 534 & 13.5 \\
\hline 0912 & $\mathrm{CF}$ & Environmental & 19960712 & 1112 & 4.5 & .4 & 7.2 & 820 & 13 \\
\hline 0913 & $\mathrm{CF}$ & Environmental & 19960712 & 1507 & 4.2 & 5.9 & 7.1 & 1,380 & 13 \\
\hline 1001 & $\mathrm{CF}$ & Environmental & 19950728 & 1100 & $<.05$ & $<.1$ & 8.2 & 780 & 14 \\
\hline 1001 & $\mathrm{CF}$ & Environmental & 19960718 & 1415 & .76 & -- & 7.0 & 1,070 & 12.5 \\
\hline 1002 & UF & Environmental & 19950728 & 1130 & $* 7.5$ & 4.6 & 6.9 & 1,050 & 12 \\
\hline 1002 & UF & Environmental & 19960718 & 1400 & $* .11$ & - & 8.3 & 771 & 14.5 \\
\hline 1003 & $\mathrm{CF}$ & Environmental & 19950727 & 900 & $* 1.5$ & -- & 7.5 & 605 & 14.5 \\
\hline 1003 & $\mathrm{CF}$ & Environmental & 19960712 & 1340 & $* 17$ & 2.3 & 7.1 & 947 & 12 \\
\hline 1004 & CF & Environmental & 19950725 & 1400 & 5.5 & $<.1$ & 7.1 & 1,110 & 14.5 \\
\hline 1004 & $\mathrm{CF}$ & Environmental & 19960722 & 1300 & 6.1 & $<.1$ & 7.0 & 1,140 & 14.5 \\
\hline 1005 & $\mathrm{CF}$ & Environmental & 19950726 & 0930 & 1.7 & 5.4 & 7.3 & 630 & 12.5 \\
\hline 1005 & $\mathrm{CF}$ & Environmental & 19960730 & 1400 & 1.4 & 4.8 & 7.1 & 639 & 12.5 \\
\hline 1006 & $\mathrm{CF}$ & Environmental & 19960730 & 1500 & 1.8 & 6.0 & 7.1 & 752 & 12.5 \\
\hline 1006 & $\mathrm{CF}$ & Duplicate & 19960730 & 1505 & 1.8 & 6.0 & 7.1 & 752 & 12.5 \\
\hline 1006 & $\mathrm{CF}$ & Blank & 19960730 & 1510 & .10 & -- & -- & - & -- \\
\hline 1101 & UF & Environmental & 19950724 & 1115 & 9.2 & 6.7 & 7.0 & 615 & 13 \\
\hline 1101 & UF & Environmental & 19960716 & 1200 & $* 11$ & 6.9 & 7.0 & 616 & 13.5 \\
\hline 1102 & $\mathrm{CF}$ & Environmental & 19950718 & 1500 & .05 & .5 & 7.5 & 571 & 15 \\
\hline 1102 & CF & Environmental & 19960716 & 1100 & .12 & .2 & 7.3 & 570 & 15 \\
\hline 1102 & $\mathrm{CF}$ & Duplicate & 19960716 & 1105 & .09 & .2 & 7.3 & 570 & 15 \\
\hline 1102 & $\mathrm{CF}$ & Blank & 19960716 & 1110 & .13 & -- & -- & -- & - \\
\hline 1103 & UF & Environmental & 19950718 & 1615 & 5.4 & 8.3 & 7.1 & 517 & 13 \\
\hline 1103 & UF & Environmental & 19960717 & 1630 & 5.6 & 6.2 & 6.8 & 509 & 14 \\
\hline 1104 & UF & Environmental & 19960717 & 1500 & $* 18$ & -- & 8.8 & 712 & 14 \\
\hline 1105 & UF & Environmental & 19960717 & 1530 & 8.8 & 6.7 & 7.1 & 609 & 14 \\
\hline 1106 & UF & Environmental & 19950727 & 1430 & $* 11$ & - & 7.0 & 514 & 13 \\
\hline 1106 & UF & Environmental & 19960715 & 1500 & $* 10$ & 8.2 & 6.7 & 544 & 13.5 \\
\hline 1107 & $\mathrm{CF}$ & Environmental & 19950719 & 1115 & 3.0 & 3.1 & 7.0 & 573 & 13 \\
\hline 1107 & $\mathrm{CF}$ & Duplicate & 19950719 & 1120 & 2.9 & 3.1 & 7.0 & 573 & 13 \\
\hline 1107 & $\mathrm{CF}$ & Blank & 19950719 & 1125 & .05 & - & - & - & - \\
\hline 1108 & UF & Environmental & 19950719 & 1530 & $* 23$ & 5.5 & 6.6 & 1,510 & 12 \\
\hline 1108 & UF & Environmental & 19960718 & 1000 & $* 22$ & -- & 6.7 & 1,430 & 12.5 \\
\hline 1109 & $\mathrm{CF}$ & Environmental & 19960716 & 1430 & $* 21$ & .8 & 7.2 & 1,060 & 13 \\
\hline 1110 & UF & Environmental & 19950718 & 1700 & $* 14$ & 13 & 7.0 & 1,030 & 12 \\
\hline 1110 & UF & Duplicate & 19950718 & 1705 & $* 13$ & 13 & 7.0 & 1,030 & 11 \\
\hline 1110 & UF & Blank & 19950718 & 1710 & $<.05$ & - & - & - & - \\
\hline 1110 & UF & Environmental & 19960716 & 0800 & $* 17$ & 9.4 & 6.9 & 1,040 & 12 \\
\hline 1111 & UF & Environmental & 19960904 & 1500 & 8.7 & 7.6 & 6.8 & 572 & 12 \\
\hline 1112 & UF & Environmental & 19950718 & 1745 & 5.1 & 4.0 & 7.0 & 809 & 12.5 \\
\hline 1112 & UF & Environmental & 19960703 & 1700 & 5.2 & -- & 6.7 & 603 & 12.5 \\
\hline 1113 & $\mathrm{CF}$ & Environmental & 19950719 & 1400 & $<.05$ & $<.1$ & 7.6 & 568 & 14.5 \\
\hline 1113 & $\mathrm{CF}$ & Environmental & 19960719 & 1400 & .09 & $<.1$ & 7.4 & 557 & 14 \\
\hline
\end{tabular}


Table 8. Nitrate concentrations and field measurements for water samples from the ground-water-quality monitoring network of domestic and registered wells, Upper Big Blue Natural Resources District, central Nebraska, July 1995 through September 1997--Continued

\begin{tabular}{|c|c|c|c|c|c|c|c|c|c|}
\hline $\begin{array}{c}\text { Well } \\
\text { identification } \\
\text { number }^{1} \\
\text { (fig. 8) }\end{array}$ & $\begin{array}{l}\text { Hydro- } \\
\text { geologic } \\
\text { condition }\end{array}$ & Sample type & $\begin{array}{l}\text { Sample } \\
\text { date }\end{array}$ & $\begin{array}{c}\text { Sample } \\
\text { time }\end{array}$ & $\begin{array}{l}\text { Nitrate and } \\
\text { nitrite as } \\
\text { nitrogen, } \\
\text { dissolved } \\
(\mathrm{mg} / \mathrm{L})\end{array}$ & $\begin{array}{l}\text { Oxygen, } \\
\text { dissolved, } \\
\text { field } \\
(\mathrm{mg} / \mathrm{L})\end{array}$ & $\begin{array}{c}\mathrm{pH}, \\
\text { field } \\
\text { (standard } \\
\text { units) }\end{array}$ & $\begin{array}{c}\text { Specific } \\
\text { conduct- } \\
\text { ance, field } \\
(\mu \mathrm{S} / \mathrm{cm})\end{array}$ & $\begin{array}{c}\text { Water } \\
\text { temperature } \\
\left({ }^{\circ} \mathrm{C}\right)\end{array}$ \\
\hline 1113 & $\mathrm{CF}$ & Duplicate & 19960719 & 1405 & .09 & $<.1$ & 7.4 & 557 & 14 \\
\hline 1113 & $\mathrm{CF}$ & Blank & 19960719 & 1416 & .16 & -- & -- & -- & -- \\
\hline 1114 & UF & Environmental & 19950726 & 1745 & 5.0 & 6.5 & 6.9 & 508 & 13.5 \\
\hline 1114 & UF & Environmental & 19960730 & 1100 & 4.9 & 6.0 & 6.9 & 505 & 14 \\
\hline 1115 & UF & Environmental & 19950720 & 1330 & 6.1 & 6.9 & 7.0 & 451 & 13.5 \\
\hline 1115 & UF & Environmental & 19960719 & 1300 & 5.0 & 7.1 & 6.8 & 449 & 13.5 \\
\hline 1116 & UF & Environmental & 19950724 & 1430 & 9.0 & 7.0 & 6.8 & 569 & 13 \\
\hline 1116 & UF & Environmental & 19960715 & 1230 & $* 12$ & -- & 7.2 & 576 & 13 \\
\hline 1117 & UF & Environmental & 19950724 & 1330 & 9.6 & -- & 7.2 & 602 & 13 \\
\hline 1117 & UF & Environmental & 19960718 & 1200 & 9.4 & -- & 6.6 & 602 & 13 \\
\hline 1118 & $\mathrm{CF}$ & Environmental & 19950725 & 0830 & 6.1 & 9.0 & -- & 457 & -- \\
\hline 1118 & $\mathrm{CF}$ & Environmental & 19960715 & 1130 & 5.9 & 8.9 & 6.8 & 467 & 13 \\
\hline 1119 & UF & Environmental & 19950724 & 1230 & 5.7 & -- & 7.1 & 539 & 13 \\
\hline 1119 & UF & Duplicate & 19950724 & 1235 & 5.5 & - & 7.1 & 539 & 13 \\
\hline 1119 & UF & Blank & 19950724 & 1240 & .06 & - & -- & - & - \\
\hline 1119 & UF & Environmental & 19960715 & 1600 & 7.8 & -- & 6.1 & 549 & 13.5 \\
\hline 1120 & UF & Environmental & 19950728 & 1600 & $* 14$ & 5.0 & 6.9 & 1,260 & 12.5 \\
\hline 1121 & UF & Environmental & 19950724 & 1540 & 5.8 & 6.6 & 7.1 & 530 & 12 \\
\hline 1121 & UF & Environmental & 19960718 & 1300 & 6.7 & -- & 6.9 & 552 & 12.5 \\
\hline 1122 & UF & Environmental & 19960730 & 0930 & $* 12$ & 11 & 6.7 & 691 & 13 \\
\hline 1123 & UF & Environmental & 19950724 & 1730 & 3.3 & 5.6 & 6.9 & 560 & 13 \\
\hline 1123 & UF & Environmental & 19960715 & 1400 & 3.9 & 5.7 & 6.8 & 562 & 12.5 \\
\hline 1124 & $\mathrm{CF}$ & Environmental & 19950726 & 0745 & 1.6 & 1.5 & 7.2 & 862 & 14 \\
\hline 1124 & $\mathrm{CF}$ & Environmental & 19960715 & 1000 & .37 & 3 & 6.9 & 834 & 13.5 \\
\hline 1125 & UF & Environmental & 19950724 & 1615 & 5.7 & 7.1 & 6.9 & 705 & 13.5 \\
\hline 1125 & UF & Environmental & 19960719 & 1500 & 4.6 & 7.9 & 7.1 & 687 & 13 \\
\hline 1126 & CF & Environmental & 19960717 & 0800 & .12 & 1.2 & 8.0 & 835 & 14.5 \\
\hline 1127 & UF & Environmental & 19950721 & 1000 & 1.7 & .2 & 7.0 & 540 & 12.5 \\
\hline 1127 & UF & Environmental & 19960717 & 1730 & 1.9 & -- & 6.7 & 557 & 13 \\
\hline 1201 & $\mathrm{CF}$ & Environmental & 19950727 & 1300 & .11 & 16 & 8.1 & 508 & 16 \\
\hline 1201 & $\mathrm{CF}$ & Environmental & 19960717 & 1300 & .13 & .1 & 7.3 & 512 & 13.5 \\
\hline 1201 & $\mathrm{CF}$ & Duplicate & 19960717 & 1305 & .13 & .1 & 7.3 & 512 & 13.5 \\
\hline 1201 & $\mathrm{CF}$ & Blank & 19960717 & 1310 & .08 & -- & - & - & -- \\
\hline 1202 & CF & Environmental & 19950717 & 1710 & 2.4 & 1.8 & 7.8 & 510 & 13 \\
\hline 1202 & CF & Duplicate & 19950717 & 1715 & 2.5 & 1.8 & 7.8 & 510 & 13 \\
\hline 1202 & $\mathrm{CF}$ & Blank & 19950717 & 1720 & $<.05$ & -- & - & - & - \\
\hline 1202 & CF & Environmental & 19960711 & 0955 & .21 & .3 & 6.9 & 560 & 13 \\
\hline 1203 & CF & Environmental & 19950718 & 0915 & .61 & .4 & 7.2 & 646 & 14 \\
\hline 1203 & $\mathrm{CF}$ & Environmental & 19960711 & 0920 & 1.1 & .29 & 6.7 & 625 & 13.5 \\
\hline 1204 & $\mathrm{CF}$ & Environmental & 19960717 & 1200 & 2.7 & 1.1 & 7.2 & 572 & 13.5 \\
\hline 1205 & UF & Environmental & 19950719 & 0845 & $* 16$ & 11 & 7.1 & 990 & 12.5 \\
\hline 1205 & UF & Environmental & 19960717 & 1030 & 9.6 & 4.4 & 7.0 & 793 & 13.5 \\
\hline 1205 & UF & Environmental & 19970805 & 1344 & $* 15$ & 10 & 6.8 & 895 & 12.5 \\
\hline 1206 & $\mathrm{CF}$ & Environmental & 19960717 & 1100 & 3.5 & 6.0 & 6.9 & 475 & 13 \\
\hline
\end{tabular}


Table 8. Nitrate concentrations and field measurements for water samples from the ground-water-quality monitoring network of domestic and registered wells, Upper Big Blue Natural Resources District, central Nebraska, July 1995 through September 1997--Continued

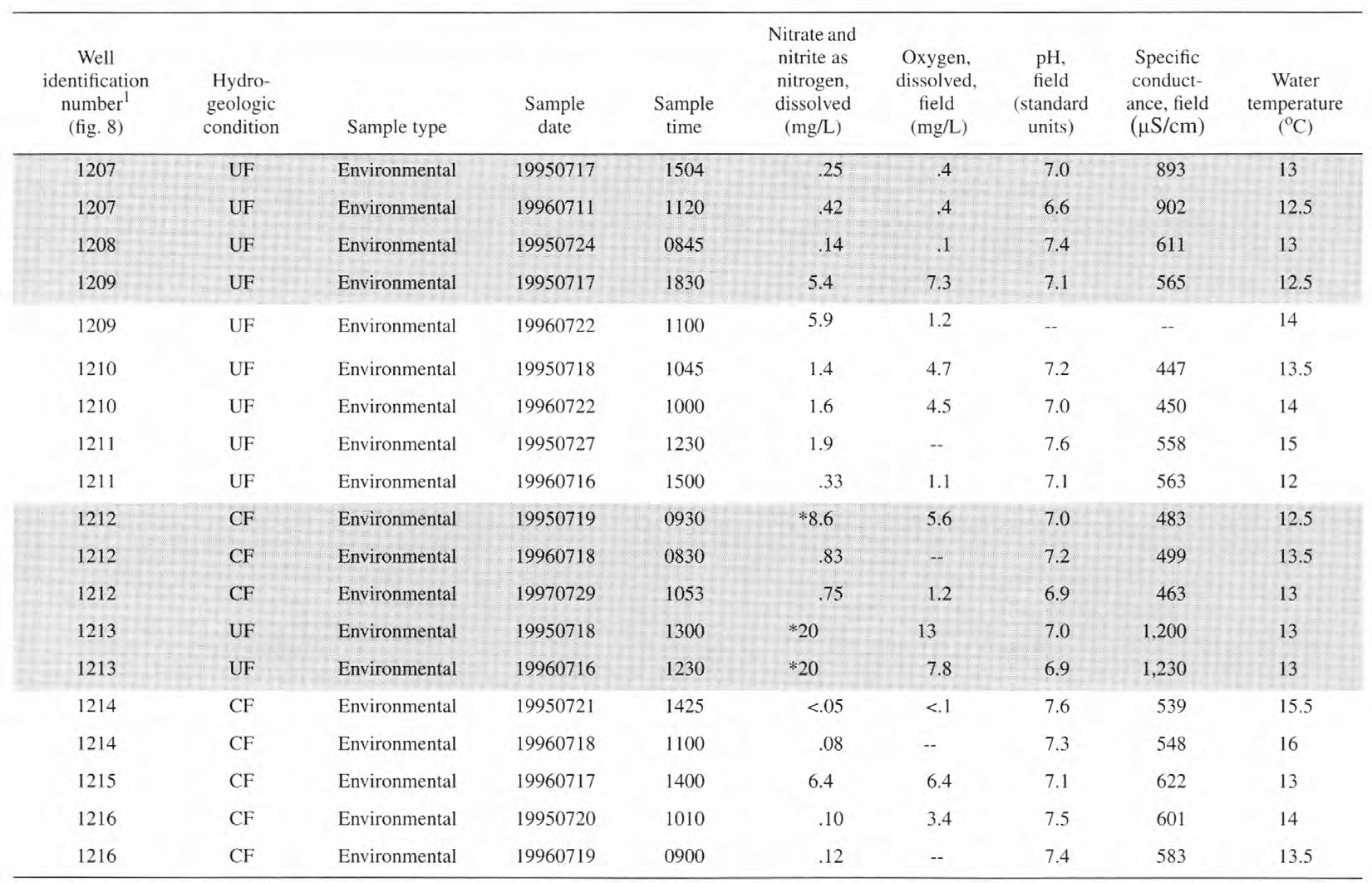

${ }^{1}$ Well identification number was assigned using management-zone number as the first two numbers; the second two numbers indicate the sequential well number. 


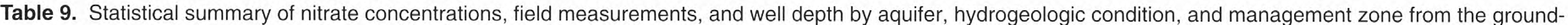

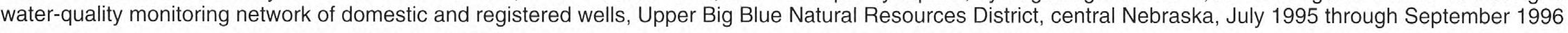

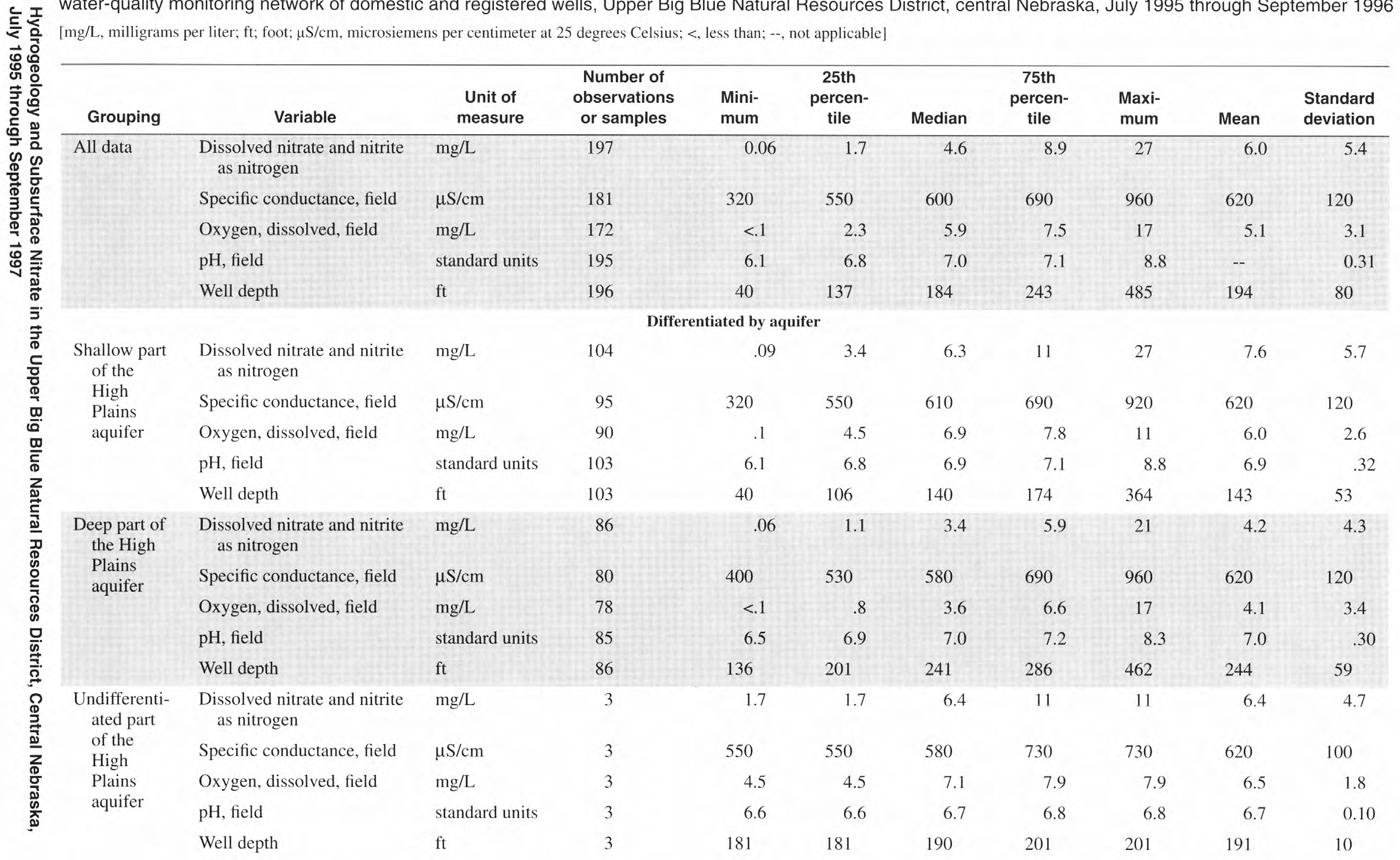


Table 9. Statistical summary of nitrate concentrations, field measurements, and well depth by aquifer, hydrogeologic condition, and management zone from the ground-water-quality monitoring network of domestic and registered wells, Upper Big Blue Natural Resources District, central Nebraska, July 1995 through September 1996--Continued

\begin{tabular}{|c|c|c|c|c|c|c|c|c|c|c|}
\hline Grouping & Variable & $\begin{array}{l}\text { Unit of } \\
\text { measure }\end{array}$ & $\begin{array}{l}\text { Number of } \\
\text { observations } \\
\text { or samples }\end{array}$ & Minimum & $\begin{array}{l}\text { 25th } \\
\text { percen- } \\
\text { tile }\end{array}$ & Median & $\begin{array}{l}\text { 75th } \\
\text { percen- } \\
\text { tile }\end{array}$ & $\begin{array}{l}\text { Maxi- } \\
\text { mum }\end{array}$ & Mean & $\begin{array}{l}\text { Standard } \\
\text { deviation }\end{array}$ \\
\hline \multirow[t]{5}{*}{$\begin{array}{l}\text { Dakota } \\
\text { aquifer }\end{array}$} & $\begin{array}{l}\text { Dissolved nitrate and nitrite } \\
\text { as nitrogen }\end{array}$ & $\mathrm{mg} / \mathrm{L}$ & 4 & 0.08 & 0.08 & 0.11 & 0.60 & 0.76 & 0.26 & 0.33 \\
\hline & Specific conductance, field & $\mu \mathrm{S} / \mathrm{cm}$ & 3 & 550 & 550 & 560 & 570 & 570 & 560 & 11 \\
\hline & Oxygen, dissolved, field & $\mathrm{mg} / \mathrm{L}$ & 1 & .2 & - & - & - & -- & -- & -- \\
\hline & $\mathrm{pH}$, field & standard units & 4 & 7.0 & 7.1 & 7.3 & 7.4 & 7.4 & 7.3 & .17 \\
\hline & Well depth & $\mathrm{ft}$ & 4 & 358 & 361 & 394 & 467 & 485 & 408 & 58 \\
\hline \multicolumn{11}{|c|}{ Differentiated by hydrogeologic condition } \\
\hline \multirow{5}{*}{$\begin{array}{l}\text { Unconfined } \\
\text { part of the } \\
\text { High } \\
\text { Plains } \\
\text { aquifer }\end{array}$} & $\begin{array}{l}\text { Dissolved nitrate and nitrite } \\
\text { as nitrogen }\end{array}$ & $\mathrm{mg} / \mathrm{L}$ & 123 & .09 & 3.0 & 6.2 & 11 & 27 & 7.3 & 5.5 \\
\hline & Specific conductance, field & $\mu \mathrm{S} / \mathrm{cm}$ & 114 & 320 & 550 & 610 & 690 & 920 & 620 & 120 \\
\hline & Oxygen, dissolved, field & $\mathrm{mg} / \mathrm{L}$ & 109 & .1 & 4.4 & 6.7 & 7.9 & 17 & 6.1 & 2.8 \\
\hline & $\mathrm{pH}$, field & standard units & 122 & 6.1 & 6.8 & 6.9 & 7.1 & 8.8 & 7.0 & .34 \\
\hline & Well depth & $\mathrm{ft}$ & 122 & 40 & 118 & 153 & 195 & 364 & 155 & 56 \\
\hline \multirow{5}{*}{$\begin{array}{l}\text { Confined part } \\
\text { of the } \\
\text { High } \\
\text { Plains } \\
\text { aquifer }\end{array}$} & $\begin{array}{l}\text { Dissolved nitrate and nitrite } \\
\text { as nitrogen }\end{array}$ & $\mathrm{mg} / \mathrm{L}$ & 74 & .06 & .67 & 2.9 & 5.0 & 21 & 3.7 & 4.3 \\
\hline & Specific conductance, field & $\mu \mathrm{S} / \mathrm{cm}$ & 67 & 400 & 530 & 580 & 680 & 960 & 610 & 130 \\
\hline & Oxygen, dissolved, field & $\mathrm{mg} / \mathrm{L}$ & 63 & $<.1$ & .50 & 2.9 & 6.3 & 9.8 & 3.5 & 3.1 \\
\hline & $\mathrm{pH}$, field & standard units & 73 & 6.5 & 6.9 & 7.1 & 7.2 & 8.0 & 7.0 & .30 \\
\hline & Well depth & $\mathrm{ft}$ & 74 & 136 & 204 & 247 & 294 & 485 & 258 & 72 \\
\hline \multicolumn{11}{|c|}{ Differentiated by management zone } \\
\hline \multirow[t]{5}{*}{$\begin{array}{l}\text { Management } \\
\text { zone } 1\end{array}$} & $\begin{array}{l}\text { Dissolved nitrate and nitrite } \\
\text { as nitrogen }\end{array}$ & $\mathrm{mg} / \mathrm{L}$ & 16 & .12 & 2.3 & 4.7 & 13 & 21 & 7.9 & 7.3 \\
\hline & Specific conductance, field & $\mu \mathrm{S} / \mathrm{cm}$ & 13 & 350 & 580 & 620 & 740 & 870 & 640 & 130 \\
\hline & Oxygen, dissolved, field & $\mathrm{mg} / \mathrm{L}$ & 15 & 1.5 & 3.9 & 7.1 & 8.0 & 9.6 & 6.2 & 2.6 \\
\hline & $\mathrm{pH}$, field & standard units & 16 & 6.7 & 6.9 & 7.1 & 7.2 & 8.3 & 7.1 & 0.36 \\
\hline & Well depth & $\mathrm{ft}$ & 16 & 125 & 164 & 205 & 255 & 364 & 212 & 61 \\
\hline
\end{tabular}


Table 9. Statistical summary of nitrate concentrations, field measurements, and well depth by aquifer, hydrogeologic condition, and management zone from the ground-water-quality monitoring network of domestic and registered wells, Upper Big Blue Natural Resources District, central Nebraska, July 1995 through

September 1996--Continued

\begin{tabular}{|c|c|c|c|c|c|c|c|c|c|c|}
\hline Grouping & Variable & $\begin{array}{l}\text { Unit of } \\
\text { measure }\end{array}$ & $\begin{array}{c}\text { Number of } \\
\text { observations } \\
\text { or samples }\end{array}$ & Minimum & $\begin{array}{l}\text { 25th } \\
\text { percen- } \\
\text { tile }\end{array}$ & Median & $\begin{array}{l}\text { 75th } \\
\text { percen- } \\
\text { tile }\end{array}$ & $\begin{array}{l}\text { Maxi- } \\
\text { mum }\end{array}$ & Mean & $\begin{array}{l}\text { Standard } \\
\text { deviation }\end{array}$ \\
\hline \multirow[t]{3}{*}{$\begin{array}{l}\text { Management } \\
\text { zone } 2\end{array}$} & $\begin{array}{l}\text { Dissolved nitrate and nitrite } \\
\text { as nitrogen }\end{array}$ & $\mathrm{mg} / \mathrm{L}$ & 15 & 0.82 & 2.6 & 5.0 & 11 & 13 & 6.2 & 4.1 \\
\hline & Oxygen, dissolved, field & $\mathrm{mg} / \mathrm{L}$ & 13 & .9 & 4.3 & 6.1 & 7.5 & 8.5 & 5.8 & 2.1 \\
\hline & $\mathrm{pH}$, field & standard units & 15 & 6.6 & 6.7 & 6.8 & 7.0 & 7.4 & 6.9 & .22 \\
\hline \multirow{4}{*}{$\begin{array}{l}\text { Management } \\
\text { zone } 3\end{array}$} & Specific conductance, field & $\mu \mathrm{S} / \mathrm{cm}$ & 20 & 430 & 540 & 600 & 670 & 870 & 620 & 120 \\
\hline & Oxygen, dissolved, field & $\mathrm{mg} / \mathrm{L}$ & 21 & .1 & 2.8 & 5.6 & 7.3 & 8.7 & 5.1 & 2.6 \\
\hline & $\mathrm{pH}$, field & standard units & 21 & 6.5 & 6.6 & 6.7 & 6.8 & 7.2 & 6.8 & .22 \\
\hline & Well depth & $\mathrm{ft}$ & 21 & 122 & 163 & 184 & 201 & 228 & 179 & 29 \\
\hline \multirow{2}{*}{$\begin{array}{l}\text { Management } \\
\text { zone } 4\end{array}$} & $\mathrm{pH}$, field & standard units & 19 & 6.8 & 6.9 & 7.0 & 7.1 & 7.5 & 7.0 & .15 \\
\hline & Well depth & $\mathrm{ft}$ & 20 & 130 & 156 & 232 & 249 & 351 & 214 & 59 \\
\hline \multirow[t]{5}{*}{$\begin{array}{l}\text { Management } \\
\text { zone } 5\end{array}$} & $\begin{array}{l}\text { Dissolved nitrate and nitrite } \\
\text { as nitrogen }\end{array}$ & $\mathrm{mg} / \mathrm{L}$ & 12 & .25 & 5.6 & 10 & 13 & 27 & 11 & 7.1 \\
\hline & Specific conductance, field & $\mu \mathrm{S} / \mathrm{cm}$ & 12 & 430 & 500 & 610 & 740 & 870 & 620 & 140 \\
\hline & Oxygen, dissolved, field & $\mathrm{mg} / \mathrm{L}$ & 12 & .8 & 6.1 & 7.1 & 8.8 & 9.6 & 6.5 & 2.9 \\
\hline & $\mathrm{pH}$, field & standard units & 12 & 6.4 & 6.9 & 7.1 & 7.2 & 7.4 & 7.0 & 0.33 \\
\hline & Well depth & $\mathrm{ft}$ & 12 & 80 & 115 & 155 & 177 & 313 & 165 & 72 \\
\hline
\end{tabular}


Table 9. Statistical summary of nitrate concentrations, field measurements, and well depth by aquifer, hydrogeologic condition, and management zone from the ground-water-quality monitoring network of domestic and registered wells, Upper Big Blue Natural Resources District, central Nebraska, July 1995 through September 1996--Continued

\begin{tabular}{|c|c|c|c|c|c|c|c|c|c|c|}
\hline Grouping & Variable & $\begin{array}{l}\text { Unit of } \\
\text { measure }\end{array}$ & $\begin{array}{l}\text { Number of } \\
\text { observations } \\
\text { or samples }\end{array}$ & Minimum & $\begin{array}{l}\text { 25th } \\
\text { percen- } \\
\text { tile }\end{array}$ & Median & $\begin{array}{l}\text { 75th } \\
\text { percen- } \\
\text { tile }\end{array}$ & $\begin{array}{l}\text { Maxi- } \\
\text { mum }\end{array}$ & Mean & $\begin{array}{l}\text { Standard } \\
\text { deviation }\end{array}$ \\
\hline \multirow[t]{5}{*}{$\begin{array}{l}\text { Management } \\
\text { zone } 6\end{array}$} & $\begin{array}{l}\text { Dissolved nitrate and nitrite } \\
\text { as nitrogen }\end{array}$ & $\mathrm{mg} / \mathrm{L}$ & 15 & 1.7 & 4.5 & 6.2 & 12 & 15 & 7.5 & 4.4 \\
\hline & Specific conductance, field & $\mu \mathrm{S} / \mathrm{cm}$ & 15 & 530 & 550 & 590 & 690 & 780 & 620 & 80 \\
\hline & Oxygen, dissolved, field & $\mathrm{mg} / \mathrm{L}$ & 15 & 2.1 & 5.9 & 7.2 & 8.9 & 11 & 7.1 & 2.3 \\
\hline & $\mathrm{pH}$, field & standard units & 15 & 6.5 & 6.7 & 6.9 & 7.1 & 7.5 & 6.9 & .26 \\
\hline & Well depth & $\mathrm{ft}$ & 15 & 63 & 106 & 171 & 181 & 230 & 153 & 51 \\
\hline \multirow[t]{5}{*}{$\begin{array}{l}\text { Management } \\
\text { zone } 7\end{array}$} & $\begin{array}{l}\text { Dissolved nitrate and nitrite } \\
\text { as nitrogen }\end{array}$ & $\mathrm{mg} / \mathrm{L}$ & 19 & .10 & 2.4 & 4.7 & 7.3 & 18 & 5.7 & 4.6 \\
\hline & Specific conductance, field & $\mu \mathrm{S} / \mathrm{cm}$ & 17 & 400 & 500 & 610 & 730 & 960 & 640 & 140 \\
\hline & Oxygen, dissolved, field & $\mathrm{mg} / \mathrm{L}$ & 18 & .2 & .5 & 3.0 & 7.0 & 9.0 & 3.6 & 3.2 \\
\hline & $\mathrm{pH}$, field & standard units & 19 & 6.5 & 6.8 & 7.0 & 7.1 & 7.4 & 7.0 & .21 \\
\hline & Well depth & $\mathrm{ft}$ & 19 & 50 & 185 & 273 & 299 & 351 & 239 & 75 \\
\hline \multirow[t]{5}{*}{$\begin{array}{l}\text { Management } \\
\text { zone } 8\end{array}$} & $\begin{array}{l}\text { Dissolved nitrate and nitrite } \\
\text { as nitrogen }\end{array}$ & $\mathrm{mg} / \mathrm{L}$ & 17 & .10 & .26 & 2.6 & 4.5 & 16 & 3.4 & 4.1 \\
\hline & Specific conductance, field & $\mu \mathrm{S} / \mathrm{cm}$ & 17 & 320 & 510 & 580 & 630 & 920 & 580 & 130 \\
\hline & Oxygen, dissolved, field & $\mathrm{mg} / \mathrm{L}$ & 15 & .1 & .5 & 3.2 & 5.6 & 9.3 & 3.5 & 3.1 \\
\hline & $\mathrm{pH}$, field & standard units & 17 & 6.5 & 6.8 & 6.9 & 7.2 & 7.4 & 7.0 & .25 \\
\hline & Well depth & $\mathrm{ft}$ & 17 & 70 & 138 & 205 & 239 & 260 & 185 & 63 \\
\hline \multirow[t]{5}{*}{$\begin{array}{l}\text { Management } \\
\text { zone } 9\end{array}$} & $\begin{array}{l}\text { Dissolved nitrate and nitrite } \\
\text { as nitrogen }\end{array}$ & $\mathrm{mg} / \mathrm{L}$ & 13 & .09 & .45 & 3.6 & 6.5 & 12 & 4.0 & 3.8 \\
\hline & Specific conductance, field & $\mu \mathrm{S} / \mathrm{cm}$ & 12 & 530 & 560 & 630 & 700 & 930 & 660 & 120 \\
\hline & Oxygen, dissolved, field & $\mathrm{mg} / \mathrm{L}$ & 12 & $<.1$ & .6 & 1.6 & 5.2 & 7.6 & 2.6 & 2.7 \\
\hline & $\mathrm{pH}$, field & standard units & 13 & 6.8 & 6.9 & 7.1 & 7.2 & 7.3 & 7.1 & .17 \\
\hline & Well depth & $\mathrm{ft}$ & 13 & 40 & 118 & 243 & 278 & 304 & 207 & 85 \\
\hline
\end{tabular}


Table 9. Statistical summary of nitrate concentrations, field measurements, and well depth by aquifer, hydrogeologic condition, and management zone from the ground-water-quality monitoring network of domestic and registered wells, Upper Big Blue Natural Resources District, central Nebraska, July 1995 through September 1996--Continued

\begin{tabular}{|c|c|c|c|c|c|c|c|c|c|c|}
\hline Grouping & Variable & $\begin{array}{l}\text { Unit of } \\
\text { measure }\end{array}$ & $\begin{array}{l}\text { Number of } \\
\text { observations } \\
\text { or samples }\end{array}$ & Minimum & $\begin{array}{l}\text { 25th } \\
\text { percen- } \\
\text { tile }\end{array}$ & Median & $\begin{array}{l}\text { 75th } \\
\text { percen- } \\
\text { tile }\end{array}$ & $\begin{array}{l}\text { Maxi- } \\
\text { mum }\end{array}$ & Mean & $\begin{array}{l}\text { Standard } \\
\text { deviation }\end{array}$ \\
\hline \multirow[t]{5}{*}{$\begin{array}{c}\text { Management } \\
\text { zone } 10\end{array}$} & $\begin{array}{l}\text { Dissolved nitrate and nitrite } \\
\text { as nitrogen }\end{array}$ & $\mathrm{mg} / \mathrm{L}$ & 6 & 0.11 & 0.60 & 1.6 & 8.8 & 17 & 4.5 & 6.5 \\
\hline & Specific conductance, field & $\mu \mathrm{S} / \mathrm{cm}$ & 4 & 640 & 670 & 760 & 900 & 950 & 780 & 130 \\
\hline & Oxygen, dissolved, field & $\mathrm{mg} / \mathrm{L}$ & 3 & 2.3 & 2.3 & 4.8 & 6.0 & 6.0 & 4.4 & 1.9 \\
\hline & $\mathrm{pH}$, field & standard units & 6 & 7.0 & 7.0 & 7.1 & 7.4 & 8.3 & 7.3 & .51 \\
\hline & Well depth & $\mathrm{ft}$ & 6 & 95 & 172 & 263 & 384 & 430 & 269 & 120 \\
\hline \multirow[t]{5}{*}{$\begin{array}{c}\text { Management } \\
\text { zone } 11\end{array}$} & $\begin{array}{l}\text { Dissolved nitrate and nitrite } \\
\text { as nitrogen }\end{array}$ & $\mathrm{mg} / \mathrm{L}$ & 27 & .09 & 3.9 & 6.7 & 12 & 22 & 8.1 & 6.2 \\
\hline & Specific conductance, field & $\mu \mathrm{S} / \mathrm{cm}$ & 23 & 450 & 550 & 570 & 620 & 840 & 600 & 98 \\
\hline & Oxygen, dissolved, field & $\mathrm{mg} / \mathrm{L}$ & 18 & .2 & 2.6 & 6.5 & 8.0 & 11 & 5.7 & 3.3 \\
\hline & $\mathrm{pH}$, field & standard units & 27 & 6.1 & 6.7 & 6.9 & 7.1 & 8.8 & 7.0 & .49 \\
\hline & Well depth & $\mathrm{ft}$ & 27 & 42 & 95 & 128 & 215 & 462 & 168 & 115 \\
\hline \multirow[t]{5}{*}{$\begin{array}{c}\text { Management } \\
\text { zone } 12\end{array}$} & $\begin{array}{l}\text { Dissolved nitrate and nitrite } \\
\text { as nitrogen }\end{array}$ & $\mathrm{mg} / \mathrm{L}$ & 16 & .08 & .16 & .97 & 5.3 & 20 & 3.3 & 5.3 \\
\hline & Specific conductance, field & $\mu \mathrm{S} / \mathrm{cm}$ & 14 & 450 & 510 & 570 & 620 & 900 & 590 & 120 \\
\hline & Oxygen, dissolved, field & $\mathrm{mg} / \mathrm{L}$ & 11 & .3 & .4 & 1.2 & 6.0 & 7.8 & 3.0 & 2.8 \\
\hline & $\mathrm{pH}$, field & standard units & 15 & 6.6 & 6.9 & 7.1 & 7.3 & 7.4 & 7.1 & .24 \\
\hline & Well depth & $\mathrm{ft}$ & 16 & 65 & 123 & 175 & 261 & 485 & 198 & 106 \\
\hline
\end{tabular}


Table 13. Nitrate concentrations and field measurements in water from nested monitoring wells, Upper Big Blue Natural Resources District, central Nebraska, July 1995 through May 1997

$\left[\mathrm{mg} / \mathrm{L}\right.$, milligrams per liter; $\mu \mathrm{S} / \mathrm{cm}$, microsiemens per centimeter at $25^{\circ} \mathrm{C} ;{ }^{\circ} \mathrm{C}$, degrees Celsius; UF, unconfined part of the High Plains aquifer; $\mathrm{CF}$, confined part of the High Plains aquifer; sampling date, yyyymmdd; --, no data; Con., continued]

\begin{tabular}{|c|c|c|c|c|c|c|c|c|}
\hline $\begin{array}{l}\text { Nested } \\
\text { moni- } \\
\text { toring } \\
\text { well } \\
\text { number }\end{array}$ & $\begin{array}{l}\text { Hydro- } \\
\text { geo- } \\
\text { logic } \\
\text { condi- } \\
\text { tion }\end{array}$ & $\begin{array}{l}\text { Sampling } \\
\text { date }\end{array}$ & Sample type & $\begin{array}{c}\text { Dissolved } \\
\text { nitrate and } \\
\text { nitrite con- } \\
\text { centration } \\
\text { as } \\
\text { nitrogen } \\
(\mathrm{mg} / \mathrm{L})\end{array}$ & $\begin{array}{l}\text { Oxygen, } \\
\text { dis- } \\
\text { solved, } \\
\text { field } \\
\text { (mg/L) }\end{array}$ & $\begin{array}{c}\mathrm{pH}, \\
\text { field } \\
\text { (stan- } \\
\text { dard } \\
\text { units) }\end{array}$ & $\begin{array}{c}\text { Specific } \\
\text { conduc- } \\
\text { tance, } \\
\text { field } \\
(\mu \mathrm{S} / \mathrm{cm})\end{array}$ & $\begin{array}{c}\text { Water } \\
\text { temper- } \\
\text { ature, } \\
\text { field } \\
\left({ }^{\circ} \mathrm{C}\right)\end{array}$ \\
\hline \multirow[t]{12}{*}{$1 \mathrm{~A}$} & UF & 19951108 & Environmental & 12 & 10 & 7.9 & 844 & 12 \\
\hline & & 19960109 & Environmental & 14 & 9.2 & 7.1 & 845 & 12.5 \\
\hline & & 19960312 & Environmental & 14 & 8.9 & 7.1 & 800 & 12.5 \\
\hline & & 19960422 & Environmental & 14 & 8.9 & 7.6 & 795 & 11.5 \\
\hline & & 19960508 & Environmental & 16 & 7.7 & 7.6 & 920 & 13.5 \\
\hline & & 19960612 & Environmental & 16 & 11 & 7.7 & 814 & 12.5 \\
\hline & & 19960724 & Environmental & 16 & 11 & 7.0 & 984 & 13.5 \\
\hline & & 19960910 & Environmental & 17 & 9.7 & 7.1 & 760 & 14 \\
\hline & & 19961112 & Environmental & 18 & 8.5 & 6.9 & 926 & 12 \\
\hline & & 19970121 & Environmental & 19 & 8.2 & 7.0 & 922 & 12 \\
\hline & & 19970317 & Environmental & 20 & 8.7 & 6.0 & 971 & 10.5 \\
\hline & & 19970519 & Environmental & 21 & - & 7.3 & 986 & 12.5 \\
\hline \multirow[t]{13}{*}{ 1B } & $\mathrm{CF}$ & 19951108 & Environmental & $<.05$ & .2 & 8.4 & 511 & 13 \\
\hline & & 19960109 & Environmental & .1 & .3 & 7.3 & 540 & 13 \\
\hline & & 19960206 & Environmental & - & 6.9 & 7.2 & 840 & 12.5 \\
\hline & & 19960312 & Environmental & $<.05$ & .3 & 7.6 & 515 & 13.5 \\
\hline & & 19960422 & Environmental & $<.05$ & 1.6 & 7.9 & 500 & 12 \\
\hline & & 19960508 & Environmental & .1 & .5 & 7.9 & 544 & 12 \\
\hline & & 19960612 & Environmental & .14 & .6 & 7.4 & 477 & 13 \\
\hline & & 19960724 & Environmental & .12 & .5 & 7.3 & 558 & 14 \\
\hline & & 19960910 & Environmental & .14 & .3 & 7.3 & 541 & 14 \\
\hline & & 19961112 & Environmental & .10 & .4 & 7.2 & 532 & 13 \\
\hline & & 19970121 & Environmental & .10 & 1.1 & 7.3 & 522 & 13 \\
\hline & & 19970317 & Environmental & .07 & 1.1 & 6.3 & 516 & 11 \\
\hline & & 19970519 & Environmental & .08 & -- & 7.6 & 509 & 13 \\
\hline \multirow[t]{11}{*}{$2 \mathrm{~A}$} & UF & 19951108 & Environmental & 6.4 & 12 & 7.8 & 622 & 12.5 \\
\hline & & 19960109 & Environmental & 6.7 & 8.3 & 6.9 & 620 & 13 \\
\hline & & 19960206 & Environmental & 6.2 & 10 & 7.0 & 600 & 13 \\
\hline & & 19960312 & Environmental & 6.6 & 8.4 & 6.9 & 580 & 13 \\
\hline & & 19960422 & Environmental & 6.1 & 11 & 7.4 & 545 & 12 \\
\hline & & 19960508 & Environmental & 6.8 & 9.2 & 7.5 & 630 & 13 \\
\hline & & 19960612 & Environmental & 6.6 & 11 & 7.5 & 559 & 13 \\
\hline & & 19960724 & Environmental & 7.1 & 10 & 6.9 & 646 & 13.5 \\
\hline & & 19960910 & Environmental & 7.4 & 10 & 7.0 & 570 & 14.5 \\
\hline & & 19961112 & Environmental & 6.9 & 8.0 & 6.8 & 615 & 12.5 \\
\hline & & 19970121 & Environmental & 6.3 & 8.0 & 7.0 & 614 & 12 \\
\hline
\end{tabular}


Table 13. Nitrate concentrations and field measurements in water from nested monitoring wells, Upper Big Blue Natural Resources District, central Nebraska, July 1995 through May 1997--Continued

\begin{tabular}{|c|c|c|c|c|c|c|c|c|}
\hline $\begin{array}{l}\text { Nested } \\
\text { moni- } \\
\text { toring } \\
\text { well } \\
\text { number }\end{array}$ & $\begin{array}{l}\text { Hydro- } \\
\text { geo- } \\
\text { logic } \\
\text { condi- } \\
\text { tion }\end{array}$ & $\begin{array}{l}\text { Sampling } \\
\text { date }\end{array}$ & Sample type & $\begin{array}{l}\text { Dissolved } \\
\text { nitrate and } \\
\text { nitrite con- } \\
\text { centration } \\
\text { as } \\
\text { nitrogen } \\
\text { (mg/L) }\end{array}$ & $\begin{array}{l}\text { Oxygen, } \\
\text { dis- } \\
\text { solved, } \\
\text { field } \\
\text { (mg/L) }\end{array}$ & $\begin{array}{c}\mathrm{pH}, \\
\text { field } \\
\text { (stan- } \\
\text { dard } \\
\text { units) }\end{array}$ & $\begin{array}{c}\text { Specific } \\
\text { conduc- } \\
\text { tance, } \\
\text { field } \\
(\mu \mathrm{S} / \mathrm{cm})\end{array}$ & $\begin{array}{c}\text { Water } \\
\text { temper- } \\
\text { ature, } \\
\text { field } \\
\left({ }^{\circ} \mathrm{C}\right)\end{array}$ \\
\hline \multirow[t]{2}{*}{ 2A Con. } & UF & 19970317 & Environmental & 6.5 & 11 & 6.8 & 643 & 11 \\
\hline & & 19970519 & Environmental & 6.1 & - & 6.9 & 574 & 13 \\
\hline \multirow[t]{12}{*}{$2 \mathrm{~B}$} & UF & 19951108 & Environmental & 2.6 & 3.8 & 7.9 & 472 & 13 \\
\hline & & 19960109 & Environmental & 3.1 & 4.0 & 7.0 & 515 & 13 \\
\hline & & 19960206 & Environmental & 3.0 & 4.1 & 7.0 & 500 & 13 \\
\hline & & 19960312 & Environmental & 2.8 & 4.0 & 6.9 & 490 & 13 \\
\hline & & 19960422 & Environmental & 2.8 & 4.1 & 7.4 & 470 & 12 \\
\hline & & 19960612 & Environmental & 3.0 & 4.7 & 7.5 & 452 & 13 \\
\hline & & 19960724 & Environmental & 3.0 & 4.3 & 7.9 & 516 & 13.5 \\
\hline & & 19960910 & Environmental & 3.0 & 4.8 & 6.9 & 241 & 14 \\
\hline & & 19961112 & Environmental & 3.7 & 3.8 & 6.8 & 522 & 13 \\
\hline & & 19970121 & Environmental & 3.6 & 4.8 & 7.1 & 515 & 12.5 \\
\hline & & 19970317 & Environmental & 3.1 & 5.0 & 6.9 & 518 & 10 \\
\hline & & 19970519 & Environmental & 2.9 & -- & 7.0 & 495 & 13 \\
\hline \multirow[t]{13}{*}{$3 \mathrm{~A}$} & UF & 19951107 & Environmental & 9.2 & 9.2 & 7.4 & 575 & 12 \\
\hline & & 19960109 & Environmental & 8.5 & 4.2 & 6.5 & 585 & 12.5 \\
\hline & & 19960207 & Environmental & 8.1 & 4.5 & 6.6 & 585 & 12.5 \\
\hline & & 19960312 & Environmental & 8.8 & 5.4 & 6.5 & 570 & 12.5 \\
\hline & & 19960423 & Environmental & 7.9 & 8.3 & 6.8 & 560 & 11 \\
\hline & & 19960509 & Environmental & 9.0 & 9.2 & 6.9 & 615 & 12.5 \\
\hline & & 19960612 & Environmental & 9.2 & 9.8 & 6.5 & 550 & 14.5 \\
\hline & & 19960724 & Environmental & - & 11 & 6.4 & 625 & 13 \\
\hline & & 19960910 & Environmental & 7.4 & 9.3 & 6.5 & 531 & 14 \\
\hline & & 19961112 & Environmental & 7.8 & 7.3 & 6.3 & 580 & 12 \\
\hline & & 19970122 & Environmental & 8.3 & 6.1 & 6.5 & 624 & 11.5 \\
\hline & & 19970318 & Environmental & 8.7 & 9.5 & 6.4 & 625 & 12 \\
\hline & & 19970519 & Environmental & 8.6 & - & 6.5 & 640 & 13 \\
\hline \multirow[t]{11}{*}{$3 B$} & UF & 19951107 & Environmental & 1.0 & 3.2 & 7.9 & 641 & 13 \\
\hline & & 19960109 & Environmental & 1.1 & 2.9 & 7.0 & 680 & 13.5 \\
\hline & & 19960207 & Environmental & 1.0 & 3.0 & 7.0 & 670 & 13.5 \\
\hline & & 19960312 & Environmental & 1.1 & 3.2 & 6.9 & 640 & 13.5 \\
\hline & & 19960423 & Environmental & 1.0 & 8.0 & 7.4 & 640 & 12 \\
\hline & & 19960509 & Environmental & 1.1 & 3.4 & 7.4 & 695 & 13 \\
\hline & & 19960612 & Environmental & 1.1 & 3.6 & 7.0 & 619 & 15 \\
\hline & & 19960724 & Environmental & 1.1 & 3.5 & 6.8 & 680 & 14.5 \\
\hline & & 19960910 & Environmental & 1.0 & 3.5 & 6.9 & 672 & 14 \\
\hline & & 19961112 & Environmental & 1.1 & 11 & 6.8 & 691 & 12.5 \\
\hline & & 19970122 & Environmental & 1.1 & 3.8 & 7.1 & 689 & 12 \\
\hline
\end{tabular}


Table 13. Nitrate concentrations and field measurements in water from nested monitoring wells, Upper Big Blue Natural Resources District, central Nebraska, July 1995 through May 1997--Continued

\begin{tabular}{|c|c|c|c|c|c|c|c|c|}
\hline $\begin{array}{c}\text { Nested } \\
\text { moni- } \\
\text { toring } \\
\text { well } \\
\text { number }\end{array}$ & $\begin{array}{l}\text { Hydro- } \\
\text { geo- } \\
\text { logic } \\
\text { condi- } \\
\text { tion }\end{array}$ & $\begin{array}{c}\text { Sampling } \\
\text { date }\end{array}$ & Sample type & $\begin{array}{c}\text { Dissolved } \\
\text { nitrate and } \\
\text { nitrite con- } \\
\text { centration } \\
\text { as } \\
\text { nitrogen } \\
\text { (mg/L) }\end{array}$ & $\begin{array}{c}\text { Oxygen, } \\
\text { dis- } \\
\text { solved, } \\
\text { field } \\
\text { (mg/L) }\end{array}$ & $\begin{array}{c}\mathrm{pH}, \\
\text { field } \\
\text { (stan- } \\
\text { dard } \\
\text { units) }\end{array}$ & $\begin{array}{c}\text { Specific } \\
\text { conduc- } \\
\text { tance, } \\
\text { field } \\
(\mu \mathrm{S} / \mathrm{cm})\end{array}$ & $\begin{array}{l}\text { Water } \\
\text { temper- } \\
\text { ature, } \\
\text { field } \\
\left({ }^{\circ} \mathrm{C}\right)\end{array}$ \\
\hline \multirow[t]{2}{*}{ 3B Con. } & UF & 19970318 & Environmental & 1.2 & 3.3 & 6.1 & 660 & 12.5 \\
\hline & & 19970519 & Environmental & 1.2 & -- & 6.9 & 664 & 13.5 \\
\hline \multirow[t]{13}{*}{$4 \mathrm{~A}$} & UF & 19951108 & Environmental & 11 & 10 & 7.9 & 619 & 12 \\
\hline & & 19960109 & Environmental & 11 & 8.8 & 6.9 & 635 & 12 \\
\hline & & 19960206 & Environmental & 11 & 6.0 & 7.0 & 650 & 12.5 \\
\hline & & 19960313 & Environmental & 12 & 5.6 & 6.8 & 610 & 13.5 \\
\hline & & 19960424 & Environmental & 11 & 11 & 7.4 & 645 & 11.5 \\
\hline & & 19960507 & Environmental & 13 & 8.9 & 7.3 & 690 & 11.5 \\
\hline & & 19960613 & Environmental & 12 & 10 & 7.4 & 896 & 14 \\
\hline & & 19960724 & Environmental & 11 & 10 & 6.9 & 986 & 13 \\
\hline & & 19960911 & Environmental & 11 & 9.4 & 6.8 & 255 & 13 \\
\hline & & 19961112 & Environmental & 12 & 8.7 & 6.7 & 785 & 12 \\
\hline & & 19970121 & Environmental & 13 & 10 & 7.1 & 881 & 12 \\
\hline & & 19970317 & Environmental & 13 & 9.0 & 6.8 & 995 & 9.5 \\
\hline & & 19970519 & Environmental & 12 & -- & 7.1 & 997 & 12.5 \\
\hline \multirow[t]{13}{*}{ 4B } & UF & 19951108 & Environmental & 3.7 & 6.5 & 7.9 & 579 & 12.5 \\
\hline & & 19960109 & Environmental & 3.8 & 6.5 & 6.9 & 600 & 12 \\
\hline & & 19960206 & Environmental & 3.6 & 6.2 & 7.0 & 590 & 12.5 \\
\hline & & 19960313 & Environmental & 3.8 & 4.5 & 6.9 & 570 & 12.5 \\
\hline & & 19960424 & Environmental & 3.6 & 6.1 & 7.0 & 600 & 12.5 \\
\hline & & 19960507 & Environmental & 3.9 & 6.3 & 7.4 & 560 & 11.5 \\
\hline & & 19960613 & Environmental & 3.6 & 6.5 & 7.4 & 550 & 14.5 \\
\hline & & 19960724 & Environmental & 4.2 & 7.1 & 6.8 & 635 & 13 \\
\hline & & 19960911 & Environmental & 4.1 & 6.5 & 6.7 & 554 & 13 \\
\hline & & 19961112 & Environmental & 3.8 & 5.4 & 6.8 & 600 & 12 \\
\hline & & 19970121 & Environmental & 3.9 & 5.0 & 7.1 & 599 & 12 \\
\hline & & 19970317 & Environmental & 4.1 & 6.2 & 6.9 & 632 & 9 \\
\hline & & 19970519 & Environmental & 3.9 & -- & 7.0 & 608 & 12.5 \\
\hline \multirow[t]{10}{*}{$5 \mathrm{~A}$} & UF & 19951108 & Environmental & 5.4 & 3.9 & 7.8 & 604 & 12.5 \\
\hline & & 19960110 & Environmental & 7.2 & 6.3 & 6.9 & 645 & 12 \\
\hline & & 19960207 & Environmental & 7.8 & 4.7 & 6.9 & 640 & 13 \\
\hline & & 19960312 & Environmental & 9.1 & 6.3 & 6.7 & 640 & 12.5 \\
\hline & & 19960424 & Environmental & 7.1 & 5.6 & 7.2 & 605 & 13 \\
\hline & & 19960507 & Environmental & 7.5 & 6.6 & 7.3 & 575 & 12 \\
\hline & & 19960612 & Environmental & 6.8 & 6.6 & 7.4 & 590 & 12 \\
\hline & & 19960724 & Envieronmental & 7.9 & 9.2 & 7.4 & 671 & 13 \\
\hline & & 19960911 & Environmental & 5.7 & 7.4 & 6.7 & 582 & 13 \\
\hline & & 19961113 & Environmental & 6.9 & 6.2 & 6.6 & 617 & 12 \\
\hline
\end{tabular}


Table 13. Nitrate concentrations and field measurements in water from nested monitoring wells, Upper Big Blue Natural Resources District, central Nebraska, July 1995 through May 1997--Continued

\begin{tabular}{|c|c|c|c|c|c|c|c|c|}
\hline $\begin{array}{c}\text { Nested } \\
\text { moni- } \\
\text { toring } \\
\text { well } \\
\text { number }\end{array}$ & $\begin{array}{l}\text { Hydro- } \\
\text { geo- } \\
\text { logic } \\
\text { condi- } \\
\text { tion }\end{array}$ & $\begin{array}{l}\text { Sampling } \\
\text { date }\end{array}$ & Sample type & $\begin{array}{c}\text { Dissolved } \\
\text { nitrate and } \\
\text { nitrite con- } \\
\text { centration } \\
\text { as } \\
\text { nitrogen } \\
\text { (mg/L) }\end{array}$ & $\begin{array}{l}\text { Oxygen, } \\
\text { dis- } \\
\text { solved, } \\
\text { field } \\
\text { (mg/L) }\end{array}$ & $\begin{array}{l}\mathrm{pH}, \\
\text { field } \\
\text { (stan- } \\
\text { dard } \\
\text { units) }\end{array}$ & $\begin{array}{c}\text { Specific } \\
\text { conduc- } \\
\text { tance, } \\
\text { field } \\
(\mu \mathrm{S} / \mathrm{cm})\end{array}$ & $\begin{array}{l}\text { Water } \\
\text { temper- } \\
\text { ature, } \\
\text { field } \\
\left({ }^{\circ} \mathrm{C}\right)\end{array}$ \\
\hline \multirow[t]{3}{*}{ 5A Con. } & UF & 19970121 & Environmental & 7.1 & 6.8 & 6.9 & 628 & 12 \\
\hline & & 19970319 & Environmental & 7.1 & 5.7 & 6.7 & 609 & 13 \\
\hline & & 19970520 & Environmental & 7.8 & -- & 6.6 & 655 & 13 \\
\hline \multirow[t]{13}{*}{$5 \mathrm{~B}$} & $\mathrm{CF}$ & 19951108 & Environmental & -- & .3 & 7.4 & 489 & 13 \\
\hline & & 19960110 & Environmental & 1.1 & 1.4 & 7.0 & 480 & 13 \\
\hline & & 19960207 & Environmental & 1.1 & 1.1 & 7.0 & 485 & 14 \\
\hline & & 19960312 & Environmental & 1.1 & .9 & 6.9 & 470 & 13.5 \\
\hline & & 19960425 & Environmental & .77 & .7 & 7.5 & 465 & 13.5 \\
\hline & & 19960507 & Environmental & 1.0 & 1.2 & 7.5 & 450 & 12.5 \\
\hline & & 19960612 & Environmental & 1.1 & .5 & 7.5 & 448 & 12.5 \\
\hline & & 19960724 & Environmental & .79 & .9 & 6.9 & 498 & 14 \\
\hline & & 19960911 & Environmental & .96 & 1.1 & 6.9 & 473 & 13.5 \\
\hline & & 19961113 & Environmental & 1.1 & 1.0 & 6.8 & 475 & 13 \\
\hline & & 19970121 & Environmental & 1.1 & 2.0 & 7.1 & 484 & 12.5 \\
\hline & & 19970319 & Environmental & 1.1 & 1.3 & 6.8 & 457 & 13.5 \\
\hline & & 19970520 & Environmental & 1.0 & -- & 6.8 & 475 & 13.5 \\
\hline \multirow[t]{13}{*}{$6 \mathrm{~A}$} & $\mathrm{CF}$ & 19951107 & Environmental & .97 & .1 & 7.6 & 635 & 13 \\
\hline & & 19960109 & Environmental & .42 & .2 & 6.4 & 640 & 13 \\
\hline & & 19960207 & Environmental & 1.1 & .1 & 7.3 & 680 & 13.5 \\
\hline & & 19960312 & Environmental & 2.3 & .1 & 7.1 & 730 & 13.5 \\
\hline & & 19960423 & Environmental & 2.7 & .4 & 7.7 & 740 & 12.5 \\
\hline & & 19960508 & Environmental & 3.5 & .2 & 7.6 & 820 & 12.5 \\
\hline & & 19960612 & Environmental & 3.4 & .7 & 6.9 & 765 & 13 \\
\hline & & 19960724 & Environmental & 4.6 & 1.0 & 7.0 & 930 & 13.5 \\
\hline & & 19960910 & Environmental & 3.4 & .3 & 7.0 & 863 & 14.5 \\
\hline & & 19961112 & Environmental & 1.9 & 0.6 & 7.0 & 706 & 13 \\
\hline & & 19970122 & Environmental & 3.1 & .8 & 7.3 & 787 & 12.5 \\
\hline & & 19970318 & Environmental & 3.5 & .9 & 6.5 & 769 & 12.5 \\
\hline & & 19970519 & Environmental & 3.1 & -- & 7.0 & 758 & 14 \\
\hline \multirow[t]{9}{*}{$6 \mathrm{~B}$} & $\mathrm{CF}$ & 19951107 & Environmental & $<.05$ & .1 & 8.1 & 554 & 13 \\
\hline & & 19960109 & Environmental & $<.05$ & .1 & 7.2 & 580 & 13.5 \\
\hline & & 19960207 & Environmental & $<.05$ & .1 & 7.2 & 580 & 13.5 \\
\hline & & 19960312 & Environmental & $<.05$ & .1 & 7.1 & 570 & 13.5 \\
\hline & & 19960423 & Environmental & $<.05$ & .2 & 7.6 & 550 & 12 \\
\hline & & 19960508 & Environmental & .06 & .3 & 7.6 & 595 & 12 \\
\hline & & 19960612 & Environmental & .06 & .3 & 6.9 & 529 & 13.5 \\
\hline & & 19960724 & Environmental & .06 & .4 & 7.0 & 585 & 14 \\
\hline & & 19960910 & Environmental & .05 & .1 & 6.9 & 549 & 14 \\
\hline
\end{tabular}


Table 13. Nitrate concentrations and field measurements in water from nested monitoring wells, Upper Big Blue Natural Resources District, central Nebraska, July 1995 through May 1997--Continued

\begin{tabular}{|c|c|c|c|c|c|c|c|c|}
\hline $\begin{array}{l}\text { Nested } \\
\text { moni- } \\
\text { toring } \\
\text { well } \\
\text { number }\end{array}$ & $\begin{array}{l}\text { Hydro- } \\
\text { geo- } \\
\text { logic } \\
\text { condi- } \\
\text { tion }\end{array}$ & $\begin{array}{l}\text { Sampling } \\
\text { date }\end{array}$ & Sample type & $\begin{array}{l}\text { Dissolved } \\
\text { nitrate and } \\
\text { nitrite con- } \\
\text { centration } \\
\text { as } \\
\text { nitrogen } \\
\text { (mg/L) }\end{array}$ & $\begin{array}{l}\text { Oxygen, } \\
\text { dis- } \\
\text { solved, } \\
\text { field } \\
\text { (mg/L) }\end{array}$ & $\begin{array}{c}\mathrm{pH}, \\
\text { field } \\
\text { (stan- } \\
\text { dard } \\
\text { units) }\end{array}$ & $\begin{array}{c}\text { Specific } \\
\text { conduc- } \\
\text { tance, } \\
\text { field } \\
(\mu \mathrm{S} / \mathrm{cm})\end{array}$ & $\begin{array}{c}\text { Water } \\
\text { temper- } \\
\text { ature, } \\
\text { field } \\
\left({ }^{\circ} \mathrm{C}\right)\end{array}$ \\
\hline \multirow[t]{4}{*}{ 6B Con. } & $\mathrm{CF}$ & 19961112 & Environmental & .06 & .7 & 6.9 & 507 & 12.5 \\
\hline & & 19970122 & Environmental & .06 & 6 & 7.2 & 581 & 12.5 \\
\hline & & 19970318 & Environmental & $<.05$ & .5 & 5.9 & 568 & 12.5 \\
\hline & & 19970519 & Environmental & $<.05$ & -- & 7.0 & 568 & 13.5 \\
\hline \multirow[t]{13}{*}{$7 \mathrm{~A}$} & UF & 19951107 & Environmental & 1.6 & 8.0 & 7.4 & 640 & - \\
\hline & & 19960110 & Environmental & 2.3 & 7.0 & 7.1 & 580 & 13 \\
\hline & & 19960206 & Environmental & 2.1 & 1.0 & 7.1 & 590 & 12.5 \\
\hline & & 19960311 & Environmental & 4.5 & 1.5 & 7.0 & 580 & 13 \\
\hline & & 19960423 & Environmental & 4.2 & 2.1 & 7.5 & 550 & 12 \\
\hline & & 19960507 & Environmental & 4.4 & 2.0 & 7.4 & 565 & 13 \\
\hline & & 19960613 & Environmental & 4.6 & 2.6 & 7.5 & 534 & 14.5 \\
\hline & & 19960725 & Environmental & 4.4 & 3.5 & 6.5 & 595 & 13 \\
\hline & & 19960909 & Environmental & 4.6 & 3.9 & 6.8 & 550 & 13 \\
\hline & & 19961113 & Environmental & 4.4 & 1.5 & 6.7 & 577 & 12.5 \\
\hline & & 19970121 & Environmental & 4.2 & 2.8 & 7.0 & 571 & 12 \\
\hline & & 19970318 & Environmental & 4.4 & 2.6 & 6.9 & 559 & 12 \\
\hline & & 19970520 & Environmental & 4.1 & - & 6.8 & 568 & 13 \\
\hline \multirow[t]{13}{*}{$7 \mathrm{~B}$} & $\mathrm{CF}$ & 19951107 & Environmental & $<.05$ & .1 & 7.4 & 522 & 13 \\
\hline & & 19960110 & Environmental & $<.05$ & .1 & 7.2 & 515 & 13 \\
\hline & & 19960206 & Environmental & $<.05$ & .1 & 7.3 & 510 & 13 \\
\hline & & 19960311 & Environmental & $<.05$ & .1 & 7.2 & 480 & 13 \\
\hline & & 19960423 & Environmental & .07 & 3.0 & 7.7 & 306 & 12 \\
\hline & & 19960507 & Environmental & 0.12 & 0.3 & 7.7 & 475 & 12 \\
\hline & & 19960613 & Environmental & .05 & .3 & 7.8 & 454 & 14.5 \\
\hline & & 19960725 & Environmental & .08 & .7 & 7.0 & 501 & 13 \\
\hline & & 19960909 & Environmental & .06 & .1 & 7.1 & 421 & 14 \\
\hline & & 19961113 & Environmental & $<.05$ & .2 & 7.1 & 498 & 12.5 \\
\hline & & 19970121 & Environmental & .06 & 1.1 & 7.3 & 500 & 12 \\
\hline & & 19970318 & Environmental & $<.05$ & .4 & 7.1 & 491 & 12.5 \\
\hline & & 19970520 & Environmental & $<.05$ & -- & 7.1 & 500 & 13 \\
\hline \multirow[t]{9}{*}{$7 C$} & $\mathrm{CF}$ & 19951107 & Environmental & $<.05$ & .1 & 7.4 & 571 & -- \\
\hline & & 19960110 & Environmental & $<.05$ & .1 & 7.2 & 565 & 13.5 \\
\hline & & 19960206 & Environmental & $<.05$ & .1 & 7.3 & 560 & 14 \\
\hline & & 19960311 & Environmental & $<.05$ & .1 & 7.2 & 530 & 14 \\
\hline & & 19960423 & Environmental & $<.05$ & .2 & 7.7 & 500 & 13 \\
\hline & & 19960507 & Environmental & .08 & .2 & 7.7 & 520 & 13.5 \\
\hline & & 19960613 & Environmental & $<.05$ & .1 & 7.7 & 505 & 16 \\
\hline & & 19960725 & Environmental & .07 & .1 & 7.0 & 568 & 14.5 \\
\hline & & 19960909 & Environmental & - & .1 & 7.1 & 439 & 15 \\
\hline
\end{tabular}


Table 13. Nitrate concentrations and field measurements in water from nested monitoring wells, Upper Big Blue Natural Resources District, central Nebraska, July 1995 through May 1997--Continued

\begin{tabular}{|c|c|c|c|c|c|c|c|c|}
\hline $\begin{array}{l}\text { Nested } \\
\text { moni- } \\
\text { toring } \\
\text { well } \\
\text { number }\end{array}$ & $\begin{array}{l}\text { Hydro- } \\
\text { geo- } \\
\text { logic } \\
\text { condi- } \\
\text { tion }\end{array}$ & $\begin{array}{l}\text { Sampling } \\
\text { date }\end{array}$ & Sample type & $\begin{array}{c}\text { Dissolved } \\
\text { nitrate and } \\
\text { nitrite con- } \\
\text { centration } \\
\text { as } \\
\text { nitrogen } \\
\text { (mg/L) }\end{array}$ & $\begin{array}{l}\text { Oxygen, } \\
\text { dis- } \\
\text { solved, } \\
\text { field } \\
\text { (mg/L) }\end{array}$ & $\begin{array}{c}\mathrm{pH}, \\
\text { field } \\
\text { (stan- } \\
\text { dard } \\
\text { units) }\end{array}$ & $\begin{array}{c}\text { Specific } \\
\text { conduc- } \\
\text { tance, } \\
\text { field } \\
(\mu \mathrm{S} / \mathrm{cm})\end{array}$ & $\begin{array}{c}\text { Water } \\
\text { temper- } \\
\text { ature, } \\
\text { field } \\
\left({ }^{\circ} \mathrm{C}\right)\end{array}$ \\
\hline \multirow[t]{4}{*}{ 7C Con. } & $\mathrm{CF}$ & 19961113 & Environmental & $<.05$ & 1.2 & 7.0 & 558 & 14 \\
\hline & & 19970121 & Environmental & .06 & 1.0 & 7.2 & 552 & 13.5 \\
\hline & & 19970318 & Environmental & $<.05$ & .7 & 7.1 & 553 & 13 \\
\hline & & 19970520 & Environmental & $<.05$ & -- & 7.1 & 552 & 14.5 \\
\hline \multirow[t]{33}{*}{$8 \mathrm{~A}$} & UF & 19951107 & Environmental & 14 & 8.8 & 7.1 & 777 & 12 \\
\hline & & 19960110 & Blank & .06 & -- & - & -- & -- \\
\hline & & 19960110 & Environmental & 15 & 5.7 & 6.6 & 610 & 12 \\
\hline & & 19960207 & Environmental & 14 & 5.7 & 6.6 & 600 & 12 \\
\hline & & 19960207 & Blank & $<.05$ & -- & -- & -- & -- \\
\hline & & 19960207 & Duplicate & 14 & 5.7 & 6.6 & 600 & 12 \\
\hline & & 19960311 & Environmental & 15 & 7.5 & 6.5 & 560 & 12.5 \\
\hline & & 19960311 & Blank & .05 & -- & -- & -- & -- \\
\hline & & 19960311 & Duplicate & 15 & 7.5 & 6.5 & 560 & 12.5 \\
\hline & & 19960423 & Duplicate & 14 & 9.4 & 6.7 & 550 & 11.5 \\
\hline & & 19960423 & Blank & $<.05$ & - & -- & -- & -- \\
\hline & & 19960423 & Environmental & 14 & 9.3 & 6.4 & 550 & 11.5 \\
\hline & & 19960509 & Environmental & 15 & 8.7 & 6.9 & 610 & 12.5 \\
\hline & & 19960509 & Blank & .07 & -- & -- & -- & -- \\
\hline & & 19960509 & Duplicate & 15 & 8.7 & 6.9 & 610 & 12.5 \\
\hline & & 19960613 & Environmental & 13 & 8.9 & 7.1 & 536 & 14.5 \\
\hline & & 19960613 & Duplicate & 13 & 8.4 & 7.1 & 536 & 14.5 \\
\hline & & 19960613 & Blank & $<.05$ & -- & -- & -- & -- \\
\hline & & 19960725 & Environmental & 13 & 7.1 & 6.5 & 620 & 14 \\
\hline & & 19960725 & Duplicate & 13 & 7.1 & 6.5 & 620 & 14 \\
\hline & & 19960725 & Blank & .07 & -- & - & -- & - \\
\hline & & 19960909 & Blank & .07 & -- & - & -- & -- \\
\hline & & 19960909 & Environmental & 14 & 10.2 & 6.5 & 544 & 13 \\
\hline & & 19960909 & Duplicate & 14 & 10.2 & 6.5 & 544 & 13 \\
\hline & & 19961113 & Environmental & 13 & 6.8 & 6.4 & 609 & 11.5 \\
\hline & & 19961113 & Duplicate & 13 & 6.8 & 6.4 & 609 & 11.5 \\
\hline & & 19961113 & Blank & $<.05$ & - & - & -- & -- \\
\hline & & 19970122 & Environmental & 14 & 7.8 & 6.6 & 605 & 11.5 \\
\hline & & 19970122 & Duplicate & -- & 7.8 & 6.6 & 605 & 11.5 \\
\hline & & 19970122 & Blank & - & - & - & -- & - \\
\hline & & 19970318 & Environmental & 14 & 8.3 & 6.4 & 576 & 11.5 \\
\hline & & 19970318 & Duplicate & 14 & 8.3 & 6.4 & 576 & 11.5 \\
\hline & & 19970318 & Blank & $<.05$ & -- & -- & -- & -- \\
\hline
\end{tabular}


Table 13. Nitrate concentrations and field measurements in water from nested monitoring wells, Upper Big Blue Natural Resources District, central Nebraska, July 1995 through May 1997--Continued

\begin{tabular}{|c|c|c|c|c|c|c|c|c|}
\hline $\begin{array}{l}\text { Nested } \\
\text { moni- } \\
\text { toring } \\
\text { well } \\
\text { number }\end{array}$ & $\begin{array}{l}\text { Hydro- } \\
\text { geo- } \\
\text { logic } \\
\text { condi- } \\
\text { tion }\end{array}$ & $\begin{array}{l}\text { Sampling } \\
\text { date }\end{array}$ & Sample type & $\begin{array}{c}\text { Dissolved } \\
\text { nitrate and } \\
\text { nitrite con- } \\
\text { centration } \\
\text { as } \\
\text { nitrogen } \\
\text { (mg/L) }\end{array}$ & $\begin{array}{l}\text { Oxygen, } \\
\text { dis- } \\
\text { solved, } \\
\text { field } \\
\text { (mg/L) }\end{array}$ & $\begin{array}{c}\mathrm{pH}, \\
\text { field } \\
\text { (stan- } \\
\text { dard } \\
\text { units) }\end{array}$ & $\begin{array}{c}\text { Specific } \\
\text { conduc- } \\
\text { tance, } \\
\text { field } \\
(\mu \mathrm{S} / \mathrm{cm})\end{array}$ & $\begin{array}{c}\text { Water } \\
\text { temper- } \\
\text { ature, } \\
\text { field } \\
\left({ }^{\circ} \mathrm{C}\right)\end{array}$ \\
\hline \multirow[t]{3}{*}{ 8A Con. } & \multirow[t]{3}{*}{ UF } & 19970520 & Environmental & 12 & -- & 6.4 & 576 & 12.5 \\
\hline & & 19970520 & Duplicate & 12 & -- & 6.4 & 576 & 12.5 \\
\hline & & 19970520 & Blank & -- & -- & -- & -- & -- \\
\hline \multirow[t]{15}{*}{$8 \mathrm{~B}$} & \multirow[t]{15}{*}{ UF } & 19951107 & Environmental & 14 & 8.7 & 7.1 & 777 & 12 \\
\hline & & 19951107 & Blank & $<.05$ & -- & - & - & - \\
\hline & & 19951107 & Duplicate & 22 & 7.8 & 7.3 & 789 & 12 \\
\hline & & 19960110 & Environmental & 21 & 5.4 & 6.6 & 700 & 12 \\
\hline & & 19960207 & Environmental & 20 & 7.0 & 6.6 & 680 & 12 \\
\hline & & 19960311 & Environmental & - & 7.7 & 6.6 & 630 & 13 \\
\hline & & 19960423 & Environmental & 18 & 8.7 & 7.0 & 600 & 11.5 \\
\hline & & 19960509 & Environmental & 19 & 7.5 & 7.0 & 683 & 12.5 \\
\hline & & 19960613 & Environmental & 19 & 7.8 & 7.1 & 615 & 15 \\
\hline & & 19960725 & Environmental & 20 & 8.0 & 6.4 & 730 & 14 \\
\hline & & 19960909 & Environmental & 23 & 8.7 & 6.5 & 648 & 13.5 \\
\hline & & 19961113 & Environmental & 23 & 6.5 & 6.4 & 722 & 12 \\
\hline & & 19970122 & Environmental & 21 & 7.6 & 6.7 & 726 & 11.5 \\
\hline & & 19970318 & Environmental & 21 & 7.2 & 6.5 & 698 & 12 \\
\hline & & 19970520 & Environmental & 19 & - & 6.5 & 565 & 13 \\
\hline \multirow[t]{14}{*}{$8 \mathrm{C}$} & \multirow[t]{14}{*}{$\mathrm{CF}$} & 19951107 & Environmental & $<.05$ & .4 & 7.3 & 519 & 14.5 \\
\hline & & 19960110 & Environmental & .23 & -- & -- & -- & -- \\
\hline & & 19960110 & Duplicate & .23 & -- & -- & -- & -- \\
\hline & & 19960207 & Environmental & .19 & .2 & 7.1 & 520 & 13 \\
\hline & & 19960311 & Environmental & .26 & .1 & 7.0 & 500 & 14 \\
\hline & & 19960423 & Environmental & .16 & 2.2 & 7.5 & 480 & 12.5 \\
\hline & & 19960509 & Environmental & .22 & .2 & 7.5 & 524 & 14 \\
\hline & & 19960613 & Environmental & .22 & .2 & 7.6 & 467 & 16.5 \\
\hline & & 19960725 & Environmental & -- & .3 & 6.9 & 526 & 15 \\
\hline & & 19960909 & Environmental & .24 & .1 & 6.9 & 438 & 14 \\
\hline & & 19961113 & Environmental & .29 & .4 & 6.9 & 512 & 12.5 \\
\hline & & 19970122 & Environmental & .28 & .7 & 7.2 & 558 & 10 \\
\hline & & 19970318 & Environmental & .32 & .9 & 7.1 & 510 & 12.5 \\
\hline & & 19970520 & Environmental & .27 & -- & 6.9 & 490 & 14 \\
\hline
\end{tabular}


Table 14. Summary statistics for nitrate concentrations from nested monitoring wells, Upper Big Blue Natural Resources District, central Nebraska, November 1995 through May 1997

[mg/L, milligrams per liter; --, not applicable; UF, unconfined part of the High Plains aquifer; CF, confined part of the High Plains aquifer; --, not applicable]

\begin{tabular}{|c|c|c|c|c|c|c|}
\hline \multirow{2}{*}{$\begin{array}{l}\text { Grouping } \\
\text { or } \\
\text { well number }\end{array}$} & \multirow{2}{*}{$\begin{array}{c}\text { Hydro- } \\
\text { geologic } \\
\text { condition }\end{array}$} & \multirow{2}{*}{$\begin{array}{c}\text { Number } \\
\text { of } \\
\text { samples }\end{array}$} & \multicolumn{4}{|c|}{ Nitrate concentration $(\mathrm{mg} / \mathrm{L})$} \\
\hline & & & Mean & Minimum & Median & Maximum \\
\hline All data & -- & 226 & 5.5 & $<0.05$ & 3.6 & 23 \\
\hline $\begin{array}{l}\text { Shallow part of } \\
\text { High Plains aquifer }\end{array}$ & -- & 101 & 11 & 1.6 & 9.2 & 23 \\
\hline $\begin{array}{l}\text { Deep part of High } \\
\text { Plains aquifer }\end{array}$ & -- & 125 & 1.2 & $<.05$ & .42 & 4.6 \\
\hline $\begin{array}{l}\text { Unconfined part of } \\
\text { High Plains aquifer }\end{array}$ & UF & 139 & 8.6 & 1.0 & 7.1 & 23 \\
\hline $\begin{array}{l}\text { Confined part of } \\
\text { High Plains aquifer }\end{array}$ & $\mathrm{CF}$ & 87 & .60 & $<.05$ & .10 & 4.6 \\
\hline $1 \mathrm{~A}$ & UF & 12 & 16 & 12 & 16 & 21 \\
\hline 1B & CF & 12 & .09 & $<.05$ & .10 & .14 \\
\hline $2 \mathrm{~A}$ & UF & 13 & 6.6 & 6.1 & 6.6 & 7.4 \\
\hline $2 \mathrm{~B}$ & UF & 12 & 3.0 & 2.6 & 3.0 & 3.7 \\
\hline $3 \mathrm{~A}$ & UF & 12 & 8.5 & 7.4 & 8.6 & 9.2 \\
\hline 3B & UF & 13 & 1.1 & 1.0 & 1.1 & 1.2 \\
\hline $4 \mathrm{~A}$ & UF & 13 & 12 & 11 & 12 & 13 \\
\hline $4 B$ & UF & 13 & 3.8 & 3.6 & 3.8 & 4.2 \\
\hline $5 \mathrm{~A}$ & UF & 13 & 7.2 & 5.4 & 7.1 & 9.1 \\
\hline $5 B$ & CF & 12 & 1.0 & .77 & 1.1 & 1.1 \\
\hline $6 \mathrm{~A}$ & CF & 13 & 2.6 & .42 & 3.1 & 4.6 \\
\hline $6 \mathrm{~B}$ & $\mathrm{CF}$ & 13 & .05 & $<.05$ & .05 & .06 \\
\hline $7 \mathrm{~A}$ & UF & 13 & 3.8 & 1.6 & 4.4 & 4.6 \\
\hline $7 \mathrm{~B}$ & CF & 13 & .06 & $<.05$ & .05 & .12 \\
\hline $7 \mathrm{C}$ & $\mathrm{CF}$ & 12 & .06 & $<.05$ & .05 & .08 \\
\hline $8 \mathrm{~A}$ & UF & 13 & 14 & 12 & 14 & 15 \\
\hline $8 \mathrm{~B}$ & UF & 12 & 20 & 14 & 20 & 23 \\
\hline $8 \mathrm{C}$ & $\mathrm{CF}$ & 12 & .23 & $<.05$ & .24 & .32 \\
\hline
\end{tabular}


Table 15. Alkalinity, and major-ion and tritium concentrations in water samples from nested monitoring wells, Upper Big Blue Natural Resources District, central Nebraska, September 1996

[Site ID, nested monitoring well number; ${ }^{\circ} \mathrm{C}$, degrees Celsius; mg/L, milligrams per liter; $\mu \mathrm{g} / \mathrm{L}$, micrograms per liter; pCi/L, picocuries per liter; <, less than; --, no data]

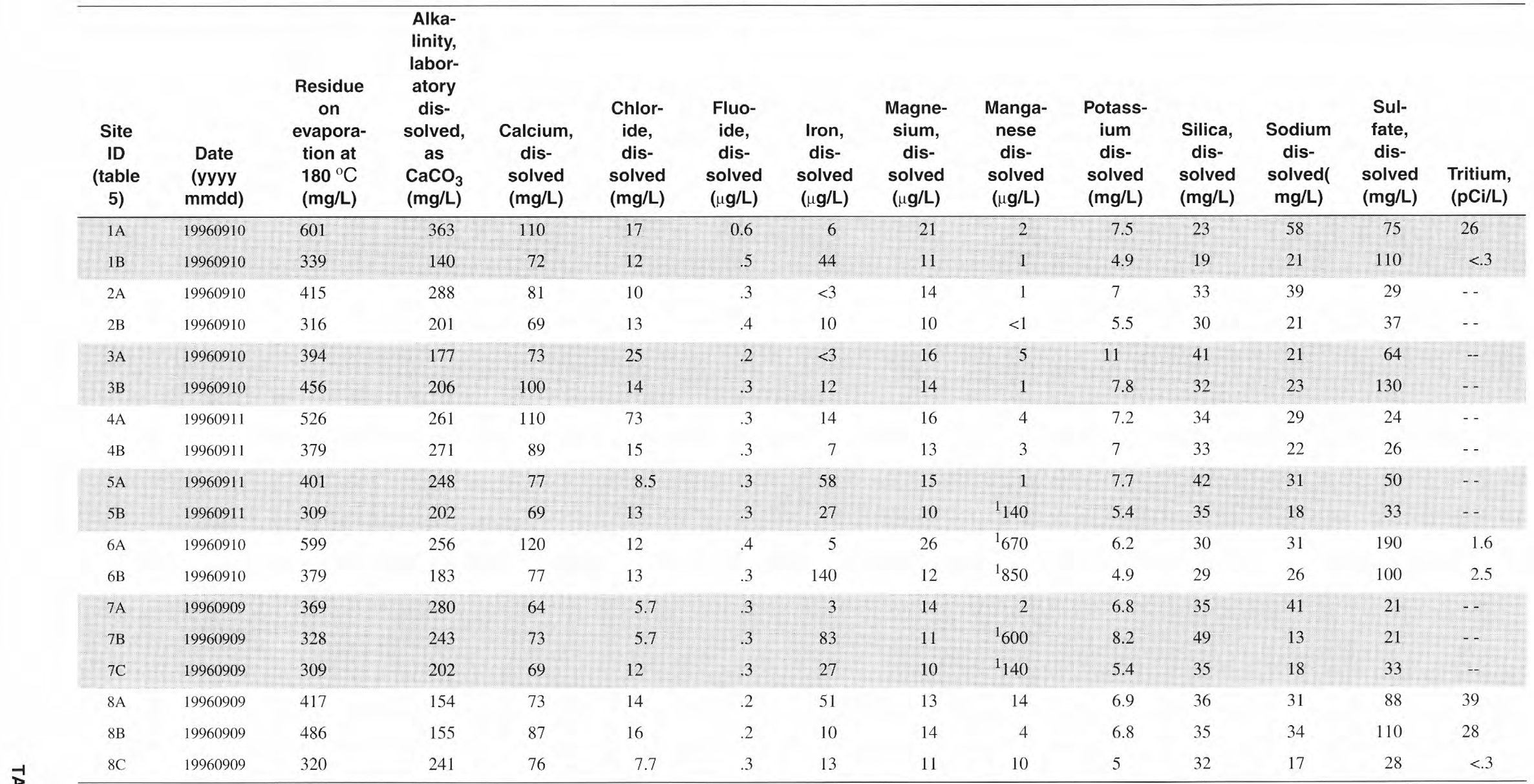


Table 16. Nitrate concentrations in unsaturated-zone sediment samples collected from selected subsites, Upper Big Blue Natural Resources District, central Nebraska, November 1995 through March 1997

[Subsite number, DH denotes deep hole, the number denotes the nested monitoring well site number, and the letters denote the respective subsite; Sample site identification number, the number denotes the nested monitoring well site number, the first letter denotes the subsite identification, the two subsequent numbers denote soil sample, and the remaining two numbers denote the sequential sample number which varies by depth]

\begin{tabular}{|c|c|c|c|c|}
\hline Subsite number & $\begin{array}{c}\text { Sample site } \\
\text { identification number }\end{array}$ & $\begin{array}{l}\text { Date of sample } \\
\text { collection } \\
\text { (yyyymmdd) }\end{array}$ & $\begin{array}{l}\text { Depth of sample } \\
\text { collection } \\
\text { (feet) }\end{array}$ & $\begin{array}{c}\text { Total nitrate } \\
\text { concentration } \\
\text { (milligrams per } \\
\text { kilogram) }\end{array}$ \\
\hline \multicolumn{5}{|c|}{ November-December 1995 sampling } \\
\hline \multirow[t]{12}{*}{ DH1 } & $1 \mathrm{SS} 01$ & 19951128 & 0.25 & 6.9 \\
\hline & $1 \mathrm{SS} 02$ & 19951128 & 1.5 & 3.5 \\
\hline & $1 \mathrm{SS} 03$ & 19951128 & 3.5 & 4.5 \\
\hline & $1 \mathrm{SS} 04$ & 19951128 & 5.5 & 7 \\
\hline & $1 \mathrm{SS} 05$ & 19951128 & 9.5 & 4.4 \\
\hline & $1 \mathrm{SS} 06$ & 19951128 & 15 & 4.3 \\
\hline & $1 \mathrm{SS} 07$ & 19951128 & 20 & 6.3 \\
\hline & $1 \mathrm{SS} 08$ & 19951128 & 30 & 5.3 \\
\hline & $1 \mathrm{SS} 09$ & 19951128 & 40 & 5 \\
\hline & $1 \mathrm{SS} 10$ & 19951128 & 50 & 8.6 \\
\hline & $1 \mathrm{SS} 11$ & 19951128 & 60 & 14 \\
\hline & $1 \mathrm{SS} 12$ & 19951128 & 65 & 5 \\
\hline \multirow[t]{13}{*}{$\mathrm{DH} 2 \mathrm{X}$} & $2 \mathrm{SS} 01$ & 19951128 & 1.0 & 30 \\
\hline & $2 \mathrm{ASS} 02$ & 19951128 & 3.0 & 11 \\
\hline & $2 \mathrm{ASS} 03$ & 19951128 & 4.5 & 8 \\
\hline & 2ASS04 & 19951128 & 10 & 2.6 \\
\hline & 2ASS05 & 19951128 & 15 & 3.2 \\
\hline & 2ASS06 & 19951128 & 20 & 4.6 \\
\hline & 2ASS07 & 19951128 & 25 & 11 \\
\hline & 2ASS08 & 19951128 & 35 & 9.8 \\
\hline & 2ASS09 & 19951128 & 45 & .5 \\
\hline & 2ASS 10 & 19951128 & 54 & 1.8 \\
\hline & 2ASS11 & 19951128 & 64 & 1.7 \\
\hline & 2ASS12 & 19951128 & 74 & 1.2 \\
\hline & $2 \mathrm{ASS} 13$ & 19951128 & 85 & 3.1 \\
\hline \multirow[t]{12}{*}{$\mathrm{DH} 2 \mathrm{Y}$} & 2BSS01 & 19951129 & 1.0 & 10 \\
\hline & $2 \mathrm{BSS} 02$ & 19951129 & 3.0 & 4.8 \\
\hline & 2BSS03 & 19951129 & 5.0 & 7.3 \\
\hline & 2BSS04 & 19951129 & 10 & 1.8 \\
\hline & 2BSS05 & 19951129 & 15 & 4.9 \\
\hline & 2BSS06 & 19951129 & 20 & 8.8 \\
\hline & 2BSS07 & 19951129 & 22 & 13 \\
\hline & 2BSS08 & 19951129 & 35 & 3.9 \\
\hline & 2BSS09 & 19951129 & 45 & 3.7 \\
\hline & 2BSS 10 & 19951129 & 50 & - \\
\hline & 2BSS11 & 19951129 & 55 & -- \\
\hline & 2BSS 12 & 19951129 & 65 & 1.2 \\
\hline
\end{tabular}


Table 16. Nitrate concentrations in unsaturated-zone sediment samples collected from selected subsites, Upper Big Blue Natural Resources District, central Nebraska, November 1995 through March 1997--Continued

\begin{tabular}{|c|c|c|c|c|}
\hline Subsite number & $\begin{array}{c}\text { Sample site } \\
\text { identification number }\end{array}$ & $\begin{array}{c}\text { Date of sample } \\
\text { collection } \\
\text { (yyyymmdd) }\end{array}$ & $\begin{array}{l}\text { Depth of sample } \\
\text { collection } \\
\text { (feet) }\end{array}$ & $\begin{array}{c}\text { Total nitrate } \\
\text { concentration } \\
\text { (milligrams per } \\
\text { kilogram) }\end{array}$ \\
\hline \multirow[t]{2}{*}{ DH2Y Con. } & 2BSS13 & 19951129 & 75 & 1.6 \\
\hline & 2BSS14 & 19951129 & 85 & 2.3 \\
\hline \multirow[t]{8}{*}{ DH3X } & 3ASS01 & 19951204 & 1.0 & 3.2 \\
\hline & $3 \mathrm{ASSO} 2$ & 19951204 & 3.0 & 2.8 \\
\hline & $3 \mathrm{ASS} 03$ & 19951204 & 5.0 & 7.1 \\
\hline & $3 \mathrm{ASSO} 04$ & 19951204 & 10 & 6.0 \\
\hline & 3ASS05 & 19951204 & 15 & 3.6 \\
\hline & 3ASS06 & 19951204 & 20 & 3.5 \\
\hline & 3ASS07 & 19951204 & 25 & 4.0 \\
\hline & 3ASS08 & 19951204 & 35 & 6.0 \\
\hline \multirow[t]{8}{*}{ DH3Y } & 3BSS01 & 19951205 & 1.0 & $<.05$ \\
\hline & 3BSSO2 & 19951205 & 3.0 & .93 \\
\hline & $3 \mathrm{BSSO} 03$ & 19951205 & 5.0 & .93 \\
\hline & 3BSS04 & 19951205 & 10 & 1.9 \\
\hline & $3 \mathrm{BSS} 05$ & 19951205 & 14 & 1.1 \\
\hline & 3BSS06 & 19951205 & 20 & 1.1 \\
\hline & 3BSS07 & 19951205 & 30 & .9 \\
\hline & 3BSS08 & 19951205 & 40 & 3.1 \\
\hline \multirow[t]{12}{*}{ DH4X } & 4ASS01 & 19951128 & 1.0 & 4.6 \\
\hline & 4ASSO2 & 19951128 & 3.0 & 2.0 \\
\hline & 4ASSO3 & 19951128 & 5.0 & 2.7 \\
\hline & 4ASSO4 & 19951128 & 10 & 2.0 \\
\hline & 4ASS05 & 19951128 & 15 & 2.2 \\
\hline & 4ASS06 & 19951128 & 20 & -- \\
\hline & 4ASS07 & 19951128 & 25 & 3.3 \\
\hline & 4ASS08 & 19951128 & 30 & 3.1 \\
\hline & 4ASS09 & 19951128 & 40 & 2.9 \\
\hline & 4ASS10 & 19951128 & 50 & 1.6 \\
\hline & 4ASS11 & 19951128 & 60 & 1.1 \\
\hline & $4 \mathrm{ASS} 12$ & 19951128 & 70 & 1.2 \\
\hline \multirow[t]{9}{*}{ DH4Y } & 4BSS01 & 19951130 & 1.0 & 5.2 \\
\hline & 4BSSO2 & 19951130 & 3.0 & 1.2 \\
\hline & 4BSSO3 & 19951130 & 5.0 & 1.2 \\
\hline & 4BSS04 & 19951130 & 10 & 1.4 \\
\hline & 4BSS05 & 19951130 & 15 & 7.2 \\
\hline & 4BSS06 & 19951130 & 20 & 1.8 \\
\hline & 4BSS07 & 19951130 & 30 & 4.4 \\
\hline & 4BSS08 & 19951130 & 40 & 1.4 \\
\hline & 4BSS09 & 19951130 & 50 & 1.1 \\
\hline
\end{tabular}


Table 16. Nitrate concentrations in unsaturated-zone sediment samples collected from selected subsites, Upper Big Blue Natural Resources District, central Nebraska, November 1995 through March 1997--Continued

\begin{tabular}{|c|c|c|c|c|}
\hline Subsite number & $\begin{array}{l}\text { Sample site } \\
\text { identification number }\end{array}$ & $\begin{array}{l}\text { Date of sample } \\
\text { collection } \\
\text { (yyyymmdd) }\end{array}$ & $\begin{array}{l}\text { Depth of sample } \\
\text { collection } \\
\text { (feet) }\end{array}$ & $\begin{array}{c}\text { Total nitrate } \\
\text { concentration } \\
\text { (milligrams per } \\
\text { kilogram) }\end{array}$ \\
\hline \multirow[t]{11}{*}{ DH4Z } & 4CSS01 & 19951130 & 1.0 & 69 \\
\hline & $4 \mathrm{CSS} 02$ & 19951130 & 3.0 & 11 \\
\hline & $4 \mathrm{CSS} 03$ & 19951130 & 5.0 & 5.4 \\
\hline & $4 \mathrm{CSS} 04$ & 19951130 & 10 & 6.6 \\
\hline & $4 \mathrm{CSS} 05$ & 19951130 & 15 & 6.5 \\
\hline & $4 \mathrm{CSS} 06$ & 19951130 & 20 & 7.6 \\
\hline & $4 \mathrm{CSS} 07$ & 19951130 & 29 & 17 \\
\hline & $4 \mathrm{CSS} 08$ & 19951130 & 40 & 16 \\
\hline & $4 \mathrm{CSS} 09$ & 19951130 & 50 & 23 \\
\hline & $4 \mathrm{CSS} 10$ & 19951130 & 60 & 25 \\
\hline & $4 \mathrm{CSS} 11$ & 19951130 & 69 & 6.3 \\
\hline \multirow[t]{11}{*}{ DH5 } & $5 \mathrm{SS} 01$ & 19951201 & 1.0 & 5.0 \\
\hline & $5 \mathrm{SS} 02$ & 19951201 & 3.0 & 4.3 \\
\hline & $5 \mathrm{SS} 03$ & 19951201 & 5.0 & 7.1 \\
\hline & $5 \mathrm{SS} 04$ & 19951201 & 10 & 1.7 \\
\hline & $5 \mathrm{SS} 05$ & 19951201 & 15 & 3.3 \\
\hline & $5 \mathrm{SS} 06$ & 19951201 & 20 & 6.5 \\
\hline & $5 \mathrm{SS} 07$ & 19951201 & 30 & 3.7 \\
\hline & $5 \mathrm{SS} 08$ & 19951201 & 40 & 3.7 \\
\hline & $5 S S 09$ & 19951201 & 50 & 1.3 \\
\hline & $5 S S 10$ & 19951201 & 60 & 1.3 \\
\hline & $5 \mathrm{SS} 11$ & 19951201 & 70 & 2.1 \\
\hline \multirow[t]{7}{*}{ DH6X } & 6ASS01 & 19951204 & 1.0 & 1.9 \\
\hline & 6ASS02 & 19951204 & 3.0 & 1.5 \\
\hline & 6ASS03 & 19951204 & 5.0 & 1.5 \\
\hline & 6ASS04 & 19951204 & 10 & .84 \\
\hline & 6ASS05 & 19951204 & 15 & .95 \\
\hline & 6ASS06 & 19951204 & 20 & .67 \\
\hline & 6ASS07 & 19951204 & 30 & 1.3 \\
\hline \multirow[t]{8}{*}{ DH6Y } & 6BSS01 & 19951204 & 1.0 & 2.7 \\
\hline & 6BSS02 & 19951204 & 3.0 & 1.4 \\
\hline & 6BSS03 & 19951204 & 5.0 & 3.7 \\
\hline & 6BSS04 & 19951204 & 10 & 6.5 \\
\hline & 6BSS05 & 19951204 & 15 & 7.2 \\
\hline & 6BSS06 & 19951204 & 19 & 1.9 \\
\hline & 6BSS07 & 19951204 & 30 & 1.5 \\
\hline & 6BSS08 & 19951204 & 40 & 2.6 \\
\hline \multirow[t]{3}{*}{ DH7X } & 7ASS01 & 19951130 & 1.0 & 11 \\
\hline & 7ASS02 & 19951130 & 3.0 & 2.1 \\
\hline & 7ASS03 & 19951130 & 5.0 & 2.1 \\
\hline
\end{tabular}


Table 16. Nitrate concentrations in unsaturated-zone sediment samples collected from selected subsites, Upper Big Blue Natural Resources District, central Nebraska, November 1995 through March 1997--Continued

\begin{tabular}{|c|c|c|c|c|}
\hline Subsite number & $\begin{array}{l}\text { Sample site } \\
\text { identification number }\end{array}$ & $\begin{array}{c}\text { Date of sample } \\
\text { collection } \\
\text { (yyyymmdd) }\end{array}$ & $\begin{array}{l}\text { Depth of sample } \\
\text { collection } \\
\text { (feet) }\end{array}$ & $\begin{array}{c}\text { Total nitrate } \\
\text { concentration } \\
\text { (milligrams per } \\
\text { kilogram) }\end{array}$ \\
\hline \multirow[t]{3}{*}{ DH7X Con. } & 7ASSO4 & 19951130 & 10 & 4.4 \\
\hline & 7ASS05 & 19951130 & 15 & 1.3 \\
\hline & 7ASS06 & 19951130 & 20 & .94 \\
\hline \multirow[t]{5}{*}{ DH7Y } & 7BSS01 & 19951204 & 1.0 & 5.6 \\
\hline & $7 \mathrm{BSS} 02$ & 19951204 & 3.0 & 1.2 \\
\hline & 7BSS03 & 19951204 & 5.0 & 2.3 \\
\hline & 7BSS04 & 19951204 & 10 & 4.4 \\
\hline & 7BSS05 & 19951204 & 15 & 3.4 \\
\hline \multirow[t]{14}{*}{ DH8X } & 8SS01 & 19951201 & 1.0 & 3.5 \\
\hline & $8 \mathrm{SS} 02$ & 19951201 & 3.0 & 1.4 \\
\hline & $8 \mathrm{SS} 03$ & 19951201 & 5.0 & 3.4 \\
\hline & $8 S S 04$ & 19951201 & 10 & 11 \\
\hline & $8 \mathrm{SS} 05$ & 19951201 & 15 & 8.3 \\
\hline & $8 \mathrm{SS} 06$ & 19951201 & 20 & 6.4 \\
\hline & $8 \mathrm{SS} 07$ & 19951201 & 30 & 3.0 \\
\hline & $8 \mathrm{SS} 08$ & 19951201 & 39 & 2.2 \\
\hline & 8SS09 & 19951201 & 50 & 3.1 \\
\hline & $8 \mathrm{SS} 10$ & 19951201 & 60 & .73 \\
\hline & $8 \mathrm{SS} 11$ & 19951201 & 70 & 2.9 \\
\hline & $8 \mathrm{SS} 12$ & 19951201 & 80 & 1.7 \\
\hline & $8 \mathrm{SS} 13$ & 19951201 & 90 & 5.2 \\
\hline & \multicolumn{3}{|c|}{ December 1996-January 1997 sampling } & \\
\hline \multirow[t]{8}{*}{ DH1 } & 1ASS0196 & 19961205 & .50 & 4.5 \\
\hline & 1ASS0296 & 19961205 & 1.8 & 4.3 \\
\hline & 1ASS0396 & 19961205 & 4.5 & 6.6 \\
\hline & 1ASS0496 & 19961205 & 6.0 & 12 \\
\hline & 1ASS0596 & 19961205 & 8.0 & 8.2 \\
\hline & 1ASS0696 & 19961205 & 13 & 5.6 \\
\hline & 1ASS0796 & 19961205 & 19 & 6.0 \\
\hline & 1ASS0896 & 19961205 & 27 & 6.5 \\
\hline \multirow[t]{8}{*}{$\mathrm{DH} 2 \mathrm{X}$} & 2ASS0196 & 19961204 & .25 & 2.7 \\
\hline & 2ASS0296 & 19961204 & 1.3 & 3.2 \\
\hline & 2ASS0396 & 19961204 & 3.0 & 3.5 \\
\hline & 2ASS0496 & 19961204 & 5.0 & 3.9 \\
\hline & 2ASS0596 & 19961204 & 10 & 4.0 \\
\hline & 2ASS0696 & 19961204 & 21 & 7.7 \\
\hline & 2ASS0796 & 19961204 & 26 & 7.9 \\
\hline & 2ASS0896 & 19961204 & 31 & 5.7 \\
\hline
\end{tabular}


Table 16. Nitrate concentrations in unsaturated-zone sediment samples collected from selected subsites, Upper Big Blue Natural Resources District, central Nebraska, November 1995 through March 1997--Continued

\begin{tabular}{|c|c|c|c|c|}
\hline Subsite number & $\begin{array}{c}\text { Sample site } \\
\text { identification number }\end{array}$ & $\begin{array}{l}\text { Date of sample } \\
\text { collection } \\
\text { (yyyymmdd) }\end{array}$ & $\begin{array}{l}\text { Depth of sample } \\
\text { collection } \\
\text { (feet) }\end{array}$ & $\begin{array}{l}\text { Total nitrate } \\
\text { concentration } \\
\text { (milligrams per } \\
\text { kilogram) }\end{array}$ \\
\hline \multirow[t]{8}{*}{$\mathrm{DH} 2 \mathrm{Y}$} & 2BSS0196 & 19961204 & 1.0 & 2.6 \\
\hline & 2BSS0296 & 19961204 & 3.0 & 2.8 \\
\hline & 2BSS0396 & 19961204 & 5.0 & 3.6 \\
\hline & 2BSS0496 & 19961204 & 10 & 3.0 \\
\hline & 2BSS0596 & 19961204 & 15 & 6.3 \\
\hline & 2BSS0696 & 19961204 & 20 & 8.8 \\
\hline & 2BSS0796 & 19961204 & 25 & 8.0 \\
\hline & 2BSS0896 & 19961204 & 30 & 5.0 \\
\hline \multirow[t]{6}{*}{ DH3X } & 3ASS0196 & 19970102 & 1.0 & 4.9 \\
\hline & $3 \mathrm{ASS} 0296$ & 19970102 & 2.5 & 3.6 \\
\hline & 3ASS0396 & 19970102 & 6.0 & 6.3 \\
\hline & 3ASS0496 & 19970102 & 10 & 6.5 \\
\hline & 3ASS0596 & 19970102 & 12 & 5.7 \\
\hline & 3ASS0696 & 19970102 & 15 & 4.4 \\
\hline \multirow[t]{7}{*}{ DH3Y } & 3BSS0196 & 19970102 & .25 & 3.4 \\
\hline & 3BSS0296 & 19970102 & 1.3 & 2.4 \\
\hline & 3BSS0396 & 19970102 & 5.5 & 3.9 \\
\hline & 3BSS0496 & 19970102 & 10 & 5.3 \\
\hline & 3BSS0596 & 19970102 & 14 & 4.3 \\
\hline & 3BSS0696 & 19970102 & 20 & 3.2 \\
\hline & 3BSS0796 & 19970102 & 23 & 2.6 \\
\hline \multirow[t]{8}{*}{ DH4X } & 4ASS0196 & 19961206 & .50 & 4.0 \\
\hline & 4ASS0296 & 19961206 & 1.5 & 2.9 \\
\hline & 4ASS0396 & 19961206 & 3.0 & 2.9 \\
\hline & 4ASS0496 & 19961206 & 5.0 & 2.1 \\
\hline & 4ASS0596 & 19961206 & 10 & 1.5 \\
\hline & 4ASS0696 & 19961206 & 15 & -- \\
\hline & 4ASS0796 & 19961206 & 21 & 3.9 \\
\hline & 4ASS0896 & 19961206 & 27 & 5.4 \\
\hline \multirow[t]{8}{*}{ DH4B } & 4BSS0196 & 19961206 & .50 & 3.4 \\
\hline & 4BSS0296 & 19961206 & 1.5 & 1.7 \\
\hline & 4BSS0396 & 19961206 & 4.0 & 1.7 \\
\hline & 4BSS0496 & 19961206 & 11 & 1.8 \\
\hline & 4BSS0596 & 19961206 & 15 & 2.9 \\
\hline & 4BSS0696 & 19961206 & 21 & 2.0 \\
\hline & 4BSS0796 & 19961206 & 31 & 5.5 \\
\hline & 4BSS0896 & 19961206 & 40 & 8.0 \\
\hline
\end{tabular}


Table 16. Nitrate concentrations in unsaturated-zone sediment samples collected from selected subsites, Upper Big Blue Natural Resources District, central Nebraska, November 1995 through March 1997--Continued

\begin{tabular}{|c|c|c|c|c|}
\hline Subsite number & $\begin{array}{l}\text { Sample site } \\
\text { identification number }\end{array}$ & $\begin{array}{c}\text { Date of sample } \\
\text { collection } \\
\text { (yyyymmdd) }\end{array}$ & $\begin{array}{l}\text { Depth of sample } \\
\text { collection } \\
\text { (feet) }\end{array}$ & $\begin{array}{c}\text { Total nitrate } \\
\text { concentration } \\
\text { (milligrams per } \\
\text { kilogram) }\end{array}$ \\
\hline \multirow[t]{10}{*}{ DH4Z } & 4CSS0196 & 19961206 & .30 & 5.6 \\
\hline & 4CSS0296 & 19961206 & .80 & 10 \\
\hline & $4 \mathrm{CSS} 0396$ & 19961206 & 1.5 & 4.8 \\
\hline & $4 \mathrm{CSS} 0496$ & 19961206 & 3.0 & 3.9 \\
\hline & 4CSS0596 & 19961206 & 5.0 & 5 \\
\hline & $4 \mathrm{CSS} 0696$ & 19961206 & 10 & 5 \\
\hline & 4CSS0796 & 19961206 & 15 & -- \\
\hline & 4CSS0896 & 19961206 & 20 & 8.4 \\
\hline & 4CSS0996 & 19961206 & 25 & 8.7 \\
\hline & 4CSS1096 & 19961206 & 30 & 6.2 \\
\hline \multirow[t]{10}{*}{ DH5 } & 5SS0196 & 19961205 & .50 & 3.9 \\
\hline & 5SS0296 & 19961205 & 1.3 & 5.1 \\
\hline & $5 S S 0396$ & 19961205 & 2.3 & 8.5 \\
\hline & 5SS0496 & 19961205 & 3.5 & 7.0 \\
\hline & $5 S S 0596$ & 19961205 & 6.5 & 3.8 \\
\hline & $5 \mathrm{SS} 0696$ & 19961205 & 8.5 & 3.0 \\
\hline & $5 S S 0796$ & 19961205 & 12 & 4.9 \\
\hline & 5SS0896 & 19961205 & 16 & 4.5 \\
\hline & 5SS0996 & 19961205 & 21 & 3.0 \\
\hline & $5 S S 1096$ & 19961205 & 30 & 2.5 \\
\hline \multirow[t]{7}{*}{ DH6X } & 6ASS0196 & 19961231 & .50 & 3.1 \\
\hline & 6ASS0296 & 19961231 & 1.3 & 1.9 \\
\hline & 6ASS0396 & 19961231 & 3.0 & 1.7 \\
\hline & 6ASS0496 & 19961231 & 5.0 & 1.8 \\
\hline & 6ASS0596 & 19961231 & 9.0 & 1.6 \\
\hline & 6ASS0696 & 19961231 & 15 & 1.6 \\
\hline & 6ASS0796 & 19961231 & 21 & 1.7 \\
\hline \multirow[t]{6}{*}{ DH6Y } & 6BSS0196 & 19961231 & .50 & 4.6 \\
\hline & 6BSS0296 & 19961231 & 1.5 & 2.4 \\
\hline & 6BSS0396 & 19961231 & 3.0 & 2.4 \\
\hline & 6BSS0496 & 19961231 & 9.0 & 5.6 \\
\hline & 6BSS0596 & 19961231 & 15 & 8.5 \\
\hline & 6BSS0696 & 19961231 & 19 & 9.7 \\
\hline \multirow[t]{7}{*}{ DH7X } & 7ASS0196 & 19961230 & 0.50 & 4.1 \\
\hline & 7ASS0296 & 19961230 & 1.5 & 3.8 \\
\hline & 7ASS0396 & 19961230 & 3.0 & 2.7 \\
\hline & 7ASS0496 & 19961230 & 6.5 & 3.0 \\
\hline & 7ASS0596 & 19961230 & 10 & 3.1 \\
\hline & 7ASS0696 & 19961230 & 16 & 6.6 \\
\hline & 7ASS0796 & 19961230 & 20 & 14 \\
\hline
\end{tabular}


Table 16. Nitrate concentrations in unsaturated-zone sediment samples collected from selected subsites, Upper Big Blue Natural Resources District, central Nebraska, November 1995 through March 1997--Continued

\begin{tabular}{|c|c|c|c|c|}
\hline Subsite number & $\begin{array}{c}\text { Sample site } \\
\text { identification number }\end{array}$ & $\begin{array}{c}\text { Date of sample } \\
\text { collection } \\
\text { (yyyymmdd) }\end{array}$ & $\begin{array}{l}\text { Depth of sample } \\
\text { collection } \\
\text { (feet) }\end{array}$ & $\begin{array}{c}\text { Total nitrate } \\
\text { concentration } \\
\text { (milligrams per } \\
\text { kilogram) }\end{array}$ \\
\hline \multirow[t]{6}{*}{ DH7Y } & 7BSS0196 & 19961230 & .50 & 4.5 \\
\hline & 7BSS0296 & 19961230 & 1.5 & 3.3 \\
\hline & 7BSS0396 & 19961230 & 3.0 & 4.1 \\
\hline & 7BSS0496 & 19961230 & 5.5 & 3.8 \\
\hline & 7BSS0596 & 19961230 & 10 & 2.6 \\
\hline & 7BSS0696 & 19961230 & 14 & 3.1 \\
\hline \multirow[t]{9}{*}{ DH8X } & 8ASS0196 & 19970103 & .50 & 6.0 \\
\hline & 8ASS0296 & 19970103 & 1.5 & 6.4 \\
\hline & 8ASS0396 & 19970103 & 3 & 2.5 \\
\hline & 8ASS0496 & 19970103 & 6 & 4.5 \\
\hline & 8ASS0596 & 19970103 & 10 & 7.8 \\
\hline & 8ASS0696 & 19970103 & 16 & 7.5 \\
\hline & 8ASS0796 & 19970103 & 21 & 7.4 \\
\hline & 8ASS0896 & 19970103 & 25 & 3.8 \\
\hline & & March 1997 sam & & \\
\hline \multirow[t]{8}{*}{ DH4X } & 4ASS0196 & 19970324 & .50 & 4.6 \\
\hline & 4ASS0296 & 19970324 & 1.5 & 3.0 \\
\hline & 4ASS0396 & 19970324 & 3.0 & 2.5 \\
\hline & 4ASS0496 & 19970324 & 5.0 & 1.9 \\
\hline & 4ASS0596 & 19970324 & 10 & 2.3 \\
\hline & 4ASS0696 & 19970324 & 15 & 3.3 \\
\hline & 4ASS0796 & 19970324 & 21 & 3.0 \\
\hline & 4ASS0896 & 19970324 & 27 & -- \\
\hline \multirow[t]{7}{*}{ DH4Y } & 4BSS0196 & 19970324 & .50 & 1.7 \\
\hline & 4BSS0296 & 19970324 & 1.5 & 1.3 \\
\hline & 4BSS0396 & 19970324 & 4.0 & 1.1 \\
\hline & 4BSS0496 & 19970324 & 11 & 3.8 \\
\hline & 4BSS0596 & 19970324 & 15 & 4.5 \\
\hline & 4BSS0696 & 19970324 & 21 & 1.8 \\
\hline & 4BSS0796 & 19970324 & 31 & 2.8 \\
\hline \multirow[t]{10}{*}{ DH4Z } & 4CSS0196 & 19970324 & 0.30 & 15 \\
\hline & $4 \mathrm{CSS} 0296$ & 19970324 & .80 & 8.3 \\
\hline & $4 \mathrm{CSS} 0396$ & 19970324 & 1.5 & 6.6 \\
\hline & 4CSS0496 & 19970324 & 3.0 & 7.7 \\
\hline & 4CSS0596 & 19970324 & 5.0 & 5.3 \\
\hline & $4 \mathrm{CSS} 0696$ & 19970324 & 10 & 4.0 \\
\hline & 4CSS0796 & 19970324 & 15 & 7.9 \\
\hline & 4CSS0896 & 19970324 & 20 & 11 \\
\hline & 4CSS0996 & 19970324 & 25 & 3.9 \\
\hline & 4CSS1096 & 19970324 & 30 & 7.2 \\
\hline
\end{tabular}


Table 16. Nitrate concentrations in unsaturated-zone sediment samples collected from selected subsites, Upper Big Blue Natural Resources District, central Nebraska, November 1995 through March 1997--Continued

\begin{tabular}{|c|c|c|c|c|}
\hline Subsite number & $\begin{array}{l}\text { Sample site } \\
\text { identification number }\end{array}$ & $\begin{array}{l}\text { Date of sample } \\
\text { collection } \\
\text { (yyyymmdd) }\end{array}$ & $\begin{array}{l}\text { Depth of sample } \\
\text { collection } \\
\text { (feet) }\end{array}$ & $\begin{array}{c}\text { Total nitrate } \\
\text { concentration } \\
\text { (milligrams per } \\
\text { kilogram) }\end{array}$ \\
\hline \multirow[t]{7}{*}{ DH6X } & 6ASS0196 & 19970325 & .50 & 4.9 \\
\hline & 6ASS0296 & 19970325 & 1.3 & 1.6 \\
\hline & 6ASS0396 & 19970325 & 3.0 & 1.6 \\
\hline & 6ASS0496 & 19970325 & 5.0 & 1.3 \\
\hline & 6ASS0596 & 19970325 & 9.0 & 1.3 \\
\hline & 6ASS0696 & 19970325 & 15 & .83 \\
\hline & 6ASS0796 & 19970325 & 21 & -- \\
\hline \multirow[t]{6}{*}{ DH6Y } & 6BSS0196 & 19970325 & .50 & 5.7 \\
\hline & 6BSS0296 & 19970325 & 1.5 & 2.8 \\
\hline & 6BSS0396 & 19970325 & 3.0 & 2.9 \\
\hline & 6BSS0496 & 19970325 & 9.0 & 5.6 \\
\hline & 6BSS0596 & 19970325 & 15 & 14 \\
\hline & 6BSS0696 & 19970325 & 19 & - \\
\hline \multirow[t]{7}{*}{ DH7X } & 7ASS0196 & 19970325 & .50 & 5.8 \\
\hline & 7ASS0296 & 19970325 & 1.5 & 5.7 \\
\hline & 7ASS0396 & 19970325 & 3.0 & 4.2 \\
\hline & 7ASS0496 & 19970325 & 6.5 & 5.1 \\
\hline & 7ASS0596 & 19970325 & 10 & 12 \\
\hline & 7ASS0696 & 19970325 & 16 & 14 \\
\hline & 7ASS0796 & 19970325 & 20 & 12 \\
\hline \multirow[t]{6}{*}{ DH7Y } & 7BSS0196 & 19970325 & .50 & 11 \\
\hline & 7BSS0296 & 19970325 & 1.5 & 6.3 \\
\hline & 7BSS0396 & 19970325 & 3.0 & 2.8 \\
\hline & 7BSS0496 & 19970325 & 5.5 & 1.6 \\
\hline & 7BSS0596 & 19970325 & 10 & 2.6 \\
\hline & 7BSS0696 & 19970325 & 14 & -- \\
\hline
\end{tabular}




